ANÁLISE E OTIMIZAÇÃo DO PROCESSO DE PREDIÇÃO DE DEMANDA DE PEÇAS DE REPOSIÇÃo COM ÊNFASE NA DEMANDA INTERMITENTE

$1^{\circ}$ Edição

São José dos Pinhais

BRAZILIAN JOURNALS PUBLICAÇÕES DE PERIÓDICOS E EDITORA 2021 
Sérgio Neri de Almeida José Helvécio Martins

\section{Análise e otimização do processo de predição de demanda de peças de reposição com ênfase na demanda intermitente} $1^{\circ}$ Edição

Brazilian Journals Editora 2021 


\author{
2021 by Brazilian Journals Editora \\ Copyright (C) Brazilian Journals Editora \\ Copyright do Texto (C) 2021 Os Autores \\ Copyright da Edição (C) 2021 Brazilian Journals Editora \\ Editora Executiva: Barbara Luzia Sartor Bonfim \\ Diagramação: Sabrina Binotti \\ Edição de Arte: Sabrina Binotti \\ Revisão: Os Autores
}

O conteúdo dos artigos e seus dados em sua forma, correção e confiabilidade são de responsabilidade exclusiva dos autores. Permitido o download da obra e o compartilhamento desde que sejam atribuídos créditos aos autores, mas sem a possibilidade de alterá-la de nenhuma forma ou utilizá-la para fins comerciais.

Conselho Editorial:

Profa . Dra . Fátima Cibele Soares - Universidade Federal do Pampa, Brasil.

Prof. Dr. Gilson Silva Filho - Centro Universitário São Camilo, Brasil.

Prof. Msc. Júlio Nonato Silva Nascimento - Instituto Federal de Educação, Ciência e Tecnologia do Pará, Brasil.

Profa ${ }^{-}$. Msc. Adriana Karin Goelzer Leining - Universidade Federal do Paraná, Brasil.

Prof. Msc. Ricardo Sérgio da Silva - Universidade Federal de Pernambuco, Brasil.

Prof. Esp. Haroldo Wilson da Silva - Universidade Estadual Paulista Júlio de Mesquita Filho, Brasil.

Prof. Dr. Orlando Silvestre Fragata - Universidade Fernando Pessoa, Portugal.

Prof. Dr. Orlando Ramos do Nascimento Júnior - Universidade Estadual de Alagoas, Brasil.

Prof ${ }^{a}$. Dra . Angela Maria Pires Caniato - Universidade Estadual de Maringá, Brasil.

Profä. Drª. Genira Carneiro de Araujo - Universidade do Estado da Bahia, Brasil.

Prof. Dr. José Arilson de Souza - Universidade Federal de Rondônia, Brasil.

Profa ${ }^{-}$Msc. Maria Elena Nascimento de Lima - Universidade do Estado do Pará, Brasil.

Prof. Caio Henrique Ungarato Fiorese - Universidade Federal do Espírito Santo, Brasil.

Profạ. Drª . Silvana Saionara Gollo - Instituto Federal de Educação, Ciência e Tecnologia do Rio Grande do Sul, Brasil.

Profㅡ. Drª . Mariza Ferreira da Silva - Universidade Federal do Paraná, Brasil.

Prof. Msc. Daniel Molina Botache - Universidad del Tolima, Colômbia.

Prof. Dr. Armando Carlos de Pina Filho- Universidade Federal do Rio de Janeiro, Brasil.

Prof. Dr. Hudson do Vale de Oliveira- Instituto Federal de Educação, Ciência e Tecnologia de Roraima, Brasil.

Profa . Msc. Juliana Barbosa de Faria - Universidade Federal do Triângulo Mineiro, Brasil.

Prof ${ }^{a}$. Esp. Marília Emanuela Ferreira de Jesus - Universidade Federal da Bahia, Brasil.

Prof. Msc. Jadson Justi - Universidade Federal do Amazonas, Brasil. 
Profạ. Drª . Alexandra Ferronato Beatrici - Instituto Federal de Educação, Ciência e Tecnologia do Rio Grande do Sul, Brasil.

Prof ${ }^{a}$. Msc. Caroline Gomes Mâcedo - Universidade Federal do Pará, Brasil.

Prof. Dr. Dilson Henrique Ramos Evangelista - Universidade Federal do Sul e Sudeste do Pará, Brasil.

Prof. Dr. Edmilson Cesar Bortoletto - Universidade Estadual de Maringá, Brasil.

Prof. Msc. Raphael Magalhães Hoed - Instituto Federal do Norte de Minas Gerais, Brasil.

Profa ${ }^{a}$. Msc. Eulália Cristina Costa de Carvalho - Universidade Federal do Maranhão, Brasil.

Prof. Msc. Fabiano Roberto Santos de Lima - Centro Universitário Geraldo di Biase, Brasil.

Prof ${ }^{\text {- }}$. Dr ${ }^{\mathrm{a}}$. Gabrielle de Souza Rocha - Universidade Federal Fluminense, Brasil.

Prof. Dr. Helder Antônio da Silva, Instituto Federal de Educação do Sudeste de Minas Gerais, Brasil.

Profa . Esp. Lida Graciela Valenzuela de Brull - Universidad Nacional de Pilar,

Paraguai.

Prof‥ Drª . Jane Marlei Boeira - Universidade Estadual do Rio Grande do Sul, Brasil.

Profa. . Dra . Carolina de Castro Nadaf Leal - Universidade Estácio de Sá, Brasil.

Prof. Dr. Carlos Alberto Mendes Morais - Universidade do Vale do Rio do Sino, Brasil.

Prof. Dr. Richard Silva Martins - Instituto Federal de Educação, Ciência e Tecnologia Sul Rio Grandense, Brasil.

Prof ${ }^{a}$. Dra ${ }^{\text {. }}$. Ana Lídia Tonani Tolfo - Centro Universitário de Rio Preto, Brasil.

Prof. Dr. André Luís Ribeiro Lacerda - Universidade Federal de Mato Grosso, Brasil.

Prof. Dr. Wagner Corsino Enedino - Universidade Federal de Mato Grosso, Brasil.

Profa. Msc. Scheila Daiana Severo Hollveg - Universidade Franciscana, Brasil.

Prof. Dr. José Alberto Yemal - Universidade Paulista, Brasil.

Profa . Drª . Adriana Estela Sanjuan Montebello - Universidade Federal de São Carlos, Brasil.

Profạ. Msc. Onofre Vargas Júnior - Instituto Federal de Educação, Ciência e Tecnologia Goiano, Brasil.

Prof ${ }^{-}$. Drª . Rita de Cássia da Silva Oliveira - Universidade Estadual de Ponta Grossa, Brasil.

Prof ${ }^{a}$. Dra . Leticia Dias Lima Jedlicka - Universidade Federal do Sul e Sudeste do

Pará, Brasil.

Prof ${ }^{a}$. Dr ${ }^{a}$. Joseina Moutinho Tavares - Instituto Federal da Bahia, Brasil

Prof. Dr. Paulo Henrique de Miranda Montenegro - Universidade Federal da Paraíba, Brasil.

Prof. Dr. Claudinei de Souza Guimarães - Universidade Federal do Rio de Janeiro, Brasil.

Prof ${ }^{\text {- }}$. Dr ${ }^{\mathrm{a}}$. Christiane Saraiva Ogrodowski - Universidade Federal do Rio Grande, Brasil.

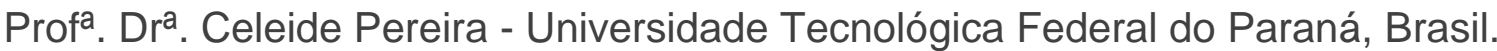

Profá. Msc. Alexandra da Rocha Gomes - Centro Universitário Unifacvest, Brasil.

Profa ${ }^{\text {- Dra }}$. Djanavia Azevêdo da Luz - Universidade Federal do Maranhão, Brasil.

Prof. Dr. Eduardo Dória Silva - Universidade Federal de Pernambuco, Brasil.

Profa. Msc. Juliane de Almeida Lira - Faculdade de Itaituba, Brasil. 
Prof. Dr. Luiz Antonio Souza de Araujo - Universidade Federal Fluminense, Brasil. Prof. Dr. Rafael de Almeida Schiavon - Universidade Estadual de Maringá, Brasil. Profa . Dra . Rejane Marie Barbosa Davim - Universidade Federal do Rio Grande do Norte, Brasil.

Prof. Msc. Salvador Viana Gomes Junior - Universidade Potiguar, Brasil.

Prof. Dr. Caio Marcio Barros de Oliveira - Universidade Federal do Maranhão, Brasil. Prof. Dr. Cleiseano Emanuel da Silva Paniagua - Instituto Federal de Educação, Ciência e Tecnologia de Goiás, Brasil.

Profa $^{a}$. Dr . Ercilia de Stefano - Universidade Federal Fluminense, Brasil. 
Dados Internacionais de Catalogação na Publicação (CIP)

A447a Almeida, Sérgio Neri de.

Análise e otimização do processo de predição de demanda de peças de reposição com ênfase na demanda intermitente / Sérgio Neri de Almeida, José Helvécio Martins. São José dos Pinhais: Editora Brazilian Journals, 2021.

$91 \mathrm{p}$.

Formato: PDF

Requisitos de sistema: Adobe Acrobat Reader

Modo de acesso: World Wide Web

Inclui: Bibliografia

ISBN: 978-65-86230-76-5

DOI: $10.35587 /$ brj.ed.0000955

1. Predição. 2. Demanda intermitente. 3. Séries temporais. 4. Estimadores. 5. Otimização. I. Almeida, Sérgio Neri de. II. Martins, José Helvécio. III. Título.

\author{
Brazilian Journals Editora \\ São José dos Pinhais - Paraná - Brasil \\ www.brazilianjournals.com.br \\ editora@brazilianjournals.com.br
}




\section{OS AUTORES}

Sérgio Neri de Almeida - Graduado em Engenharia Industrial Mecânica pelo CEFET-MG (2001), mestre em Engenharia e Gestão de Processos e Sistemas pelo IETEC (2020), na linha de pesquisa de Engenharia de Processos e Sistemas, pósgraduado em Engenharia de Manutenção pela PUC Minas (2006), MBA em Administração de Projetos com ênfase em Suprimentos pelo IETEC (2013). Experiência nas áreas de Planejamento de Manutenção e Gestão de Materiais, nos segmentos de Logística Ferroviária e Mineração. Atuação em processos nas áreas de Gestão de Mudanças e Gestão do Conhecimento no setor de Mineração.

José Helvécio Martins - Graduado em Engenharia Agrícola pela Universidade Federal de Viçosa (1979), mestre em Engenharia Agrícola pela Universidade Federal de Viçosa (1982) e doutor (PhD) em Engenharia Agrícola pela Purdue University, Estados Unidos (1988), Pós-Doutorado em Instrumentação e Controle de Processos pela Universidade de Aveiro, Portugal (2000). MBA em Gerenciamento Executivo de Projetos, UCAM/PROMINAS (2013). MBA em Engenharia de Produção, UCAM/PROMINAS (2014). Professor titular aposentado da Universidade Federal de Viçosa. Professor titular do Instituto de Educação Tecnológica - IETEC, desde 2015, no curso de Mestrado em Engenharia e Gestão de Processos e Sistemas. Experiência de 39 anos como professor e orientador de trabalhos acadêmicoscientíficos, incluindo mestrado, doutorado e pós-doutorado.

Currículo: http://lattes.cnpq.br/2582725571935270 
"Não prever, é já lamentar."

Leonardo da Vinci 


\section{DEDICATÓRIA}

Dedico este trabalho às minhas queridas esposa e filha, Mariane e Maria Alice, pela compreensão deste importante tempo de dedicação e apoio nas dificuldades, sem os quais não teria chegado até aqui. À minha irmã, Renata, pelo companheirismo e incentivo, que foram essenciais para o início desta jornada. Aos meus pais, Zélia e Francisco, a eles agradeço todo o amor e dedicação, e a oportunidade de retribuir uma pequena parcela de tudo que fizeram e fazem por mim. 


\section{AGRADECIMENTOS}

Agradeço a Deus pela força primordial que nos faz caminhar, procurando o bem e um sentido para tudo o que fazemos.

Ao Prof. Dr. José Helvécio Martins pelo exemplo de inteligência e simplicidade, a quem agradeço muito pela dedicação e confiança. Sua orientação possibilitou uma caminhada segura, onde nos momentos de dúvida, me garantiu uma inspiração sempre construtiva e otimista. Isto fez muita diferença para mim.

A todos os professores do letec pelo aprendizado e compreensão. Aos funcionários do letec em todas suas atividades.

Aos colegas do curso de Mestrado pela amizade e incentivo.

Aos colegas de trabalho pela troca de ideias e cooperação. 
RESUMO: O planejamento tem sido um fator de sucesso em muitos segmentos de negócios e está cada vez mais sendo aprimorado e assumindo maior importância nas organizações. Não é diferente no setor de manutenção cuja necessidade de implementação se torna ainda mais evidente, já que requer recursos significativos das empresas. No caso do recurso "peças de reposição", o planejamento envolve principalmente a predição de demanda, usualmente procurando um prognóstico de evento que ocorrerá no futuro com base em dados passados. Quando executado adequadamente, o planejamento pode otimizar o estoque, obtendo o nível de serviço necessário e minimizando a falta ou excesso de peças. Para um grande número de itens, nos quais o monitoramento preditivo de todas as partes é impraticável, um processo usado é a análise de séries temporais históricas com técnicas econométricas e estatísticas. No entanto, uma questão recorrente é que, devido à característica de muitos itens de reposição, sua demanda é intermitente, com muitos intervalos sem consumo intercalados com a demanda real. Essa característica aumenta a incerteza da predição, juntamente com outras incertezas, como a obsolescência de peças e mudanças nos cenários interno e externo das empresas. Para propor melhorias nesse processo, este livro procurou analisar a demanda por peças de reposição, principalmente intermitente, de uma empresa do setor de manutenção de usinas de mineração. Foram aplicados métodos de predição descritos na literatura, tanto os convencionais quanto os mais recentes, e os recursos de otimização de parâmetros presentes no algoritmo de uma rotina computacional específica disponível na plataforma R-Studio. De acordo com os métodos de estimação e otimização utilizados, foram encontradas indicações do benefício dessas técnicas, conforme as medidas de erro utilizadas. Para pesquisas futuras, sugere-se uma associação com o aprendizado de máquina, que é uma área dedicada ao desenvolvimento de algoritmos que aprendem com os dados e contribui para o processo de modelagem e predição.

PALAVRAS-CHAVE: Predição. Demanda intermitente. Séries temporais. Estimadores. Otimização. 
ABSTRACT: Planning has been a success factor in many business segments and is increasingly being improved and taking on greater importance in organizations. It is no different in the maintenance sector, whose need for implementation becomes even more evident, since it requires significant resources from companies. In the case of the "spare parts" resource, the planning mainly involves the prediction of demand, usually looking for an event forecast that will take place in the future based on past data. When properly executed, planning can optimize the inventory, obtaining the required service level and minimizing missing or excess of parts. For a large number of items, in which predictive monitoring for all parts is impractical, a process used is the analysis of historical time series with econometrics and statistics techniques. However, a recurring issue is that, due to the characteristic of many replacement items, their demand is intermittent, with many intervals without consumption interspersed with real demand. This characteristic increases the uncertainty of the prediction, along with other uncertainties, such as the obsolescence of parts and changes in internal and external scenarios in companies. In order to propose improvements to this process, this e-book sought to analyze the demand for spare parts, mostly intermittent, from a company in the sector of maintenance of mining plants. Prediction methods described in the literature, both conventional and more recent, and parameter optimization features present in the algorithm of a specific computational routine available on the R-Studio platform were applied. According to the estimation and optimization methods used, indications of the benefit of these techniques were found, according to the error measures used. However, for future research, an association with machine learning is suggested, which is an area dedicated to the development of algorithms that learn from data, and has contributed to the modeling and prediction process.

KEYWORDS: Prediction. Intermittent demand. Time series. Estimators. Optimization. 


\section{LISTA DE FIGURAS}

Figura 1- Classificação de demanda segundo Syntetos e Boylan (2005). 8

Figura 2- Exemplo de busca de $\alpha$ para uma Suavização Exponencial Simples (SES) com $l 0$ definido.

Figura 3- Exemplo busca de 10 ótimo para uma Suavização Exponencial Simples (SES) $\operatorname{com} \alpha$ definido. .18

Figura 4 - Evolução do processo de minimização pelométodo de Powell. .21

Figura 5 - Resumo da revisão da literatura. .25

Figura 6 - Organograma do processo de simulação e análise de predições. .27

Figura 7- Exemplo ilustrativo de categorização de demanda de itens da amostra....30 Figura 8 - Exemplo de aplicação de modelos de predição usando R-tsintermittent. .35 Figura 9 - Classificação da demanda da amostra.

Figura 10 - Comportamento de medidas de erro nas otimizações do Primeiro Grupo para um horizonte de predição de 6 meses, usando desvios absolutos.

Figura 11- Comparação dos erros nas predições do Primeiro Grupo de medidas para um horizonte de predição de 6 meses, usando os modelos 45

Figura 12 - Comportamento de medidas de erro nas otimizações do Segundo Grupo para um horizonte de predição de 6 meses, usando desvios quadráticos e/ou acumulativos.

Figura 13 - Comparação dos erros nas predições do Segundo Grupo de medidas para um horizonte de predição de 6 meses, usando os modelos.

Figura 14- Comparação dos erros nas predições do Primeiro Grupo de medidas para um horizonte de predição de 6 meses para demanda de alto giro, usando os modelos.

Figura 15- Comparação dos erros nas predições do Segundo Grupo de medidas para um horizonte de predição de 6 meses para demanda de alto giro, usando os modelos. .52

Figura 16 - Otimização usando o DMA (média, $\alpha \_1, \alpha \_2 ; \beta \_2-T S B$ ) .55

Figura 17 - Otimização usando o DQM (média, $\left.\alpha \_1, \alpha \_2 ; \beta \_2-T S B\right)$. .55

Figura 18 - Otimização usando o TAM (média, $\alpha \_1, \alpha \_2 ; \beta \_2-T S B$ ). .55

Figura 19 - Otimização usando o TQM (média, $\left.\alpha \_1, \alpha \_2 ; \beta \_2-T S B\right)$. .56 


\section{LISTA DE QUADROS}

Quadro 1 - Quadro de recomendações de medidas de erro segundo Wallström e Segerstedt (2010) e outra pesquisas correlatas .......................................................22

Quadro 2 - Resumo de sugestões para demanda intermitente ....................................54 


\section{LISTA DE TABELAS}

Tabela 1 - Classificação das medidas de erros DMAE, EMAE e PEEEAm, para séries intermitentes (Primeiro Grupo), usando otimização 43 Tabela 2 - Classificação das medidas de erros DMAE, EMAE e PEEEAm, para séries intermitentes (Primeiro Grupo), usando modelos de predição .44 Tabela 3 - Classificação das medidas de erros DQME, TAME e TQME, para séries intermitentes (Segundo Grupo), usando otimização .47 Tabela 4 - Classificação das medidas de erros DQME, TAME e TQME, para séries intermitentes (Segundo Grupo), usando modelos de predição Tabela 5 - Classificação das medidas de erros DMAE, EMAE e PEEEAm, para séries de alto giro - suaves e erráticas (Primeiro Grupo), usando modelos de predição 50

Tabela 6 - Classificação das medidas de erros DQME, TAME e TQME, para séries de alto giro - suaves e erráticas (Segundo Grupo), usando modelos de predição ...52 


\section{LISTA DE ABREVIATURAS E SIGLAS}

$\begin{array}{ll}\text { DMA } & \text { Desvio Médio Absoluto } \\ \text { DMAE } & \text { Desvio Médio Absoluto Escalonado } \\ \text { CRO } & \text { Método de Croston } \\ \text { EMAE } & \text { Erro Médio Absoluto Escalonado } \\ \text { DQM } & \text { Desvio Quadrático Médio } \\ \text { DQME } & \text { Desvio Quadrático Médio Escalonado } \\ \text { MMS } & \text { Método de Média Móvel Simples } \\ \text { MRO } & \text { Material de Reparo e Operação } \\ \text { PEE } & \text { Período em Estoque } \\ \text { PEEE } & \text { Período em Estoque Escalonado } \\ \text { PEEEAm } & \text { Período em Estoque Escalonado Absoluto modificado } \\ \text { SBA } & \text { Método de Aproximação Syntetos e Boylan } \\ \text { SBJ } & \text { Método de Shale, Boylan e Johnston } \\ \text { SES } & \text { Método de Suavização Exponencial Simples } \\ \text { UME } & \text { Unidade de Manutenção de Estoque UME (Stock Keeping Unit - SKU) } \\ \text { TAM } & \text { Taxa Absoluta Média } \\ \text { TAME } & \text { Taxa Absoluta Média Escalonada } \\ \text { TQM } & \text { Taxa do Quadrado da Média } \\ \text { TQME } & \text { Taxa do Quadrado da Média Escalonada } \\ \text { TSB } & \text { Método Teunter, Sani e Babai }\end{array}$


SUMÁRIO

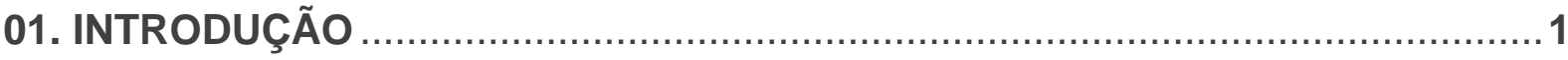

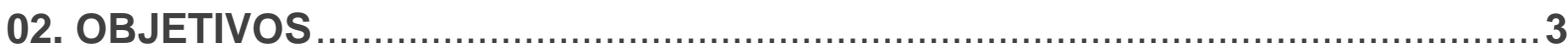

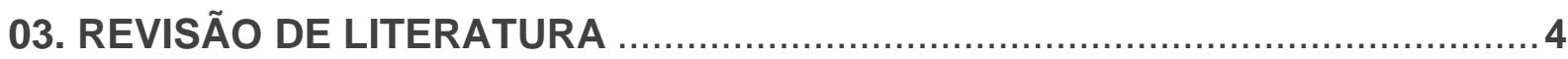

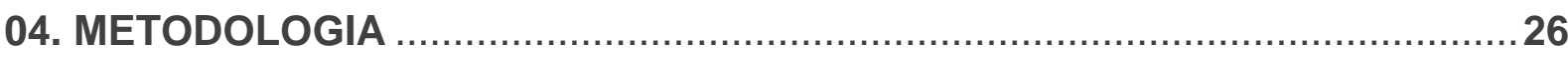

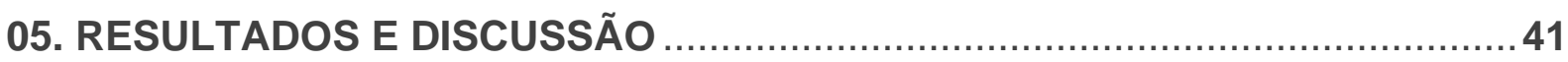

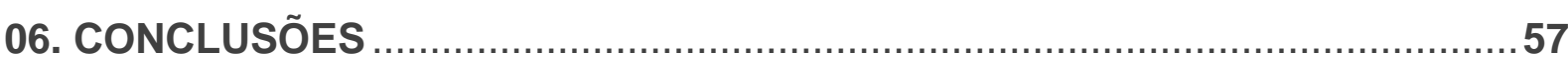

APÊNDICE A - Classificação de erros de otimizações (demanda intermitente) ......61

APÊNDICE B - Classificação de erros dos modelos (demanda intermitente) ..........65

APÊNDICE C - Classificação de erros de otimizações (demanda alto giro) ............68

APÊNDICE D - Classificação de erros dos modelos (demanda alto giro) ................72 


\section{INTRODUÇÃO}

A busca pelo tema do presente trabalho possui origem em minha experiência profissional, onde observações de iniciativas de gestão de estoques ao longo do tempo mostraram-se cíclicas. A curiosidade por buscar, por meio de um estudo mais aprofundado, alternativas para minimizar a necessidade destas ações recorrentes, inspirou a pesquisa deste livro. O tema proposto é a predição de demanda intermitente e as ações recorrentes são iniciativas de redução do inventário.

A predição de demanda é um processo essencial para tomada de decisão em diversos seguimentos de negócio. É aplicável em atividades como planejamento de curto prazo, planejamento de estoques, predição de vendas, estimativas estratégicas de longo prazo, dentre outras. O trabalho de predição, no entanto, não é simples quando realizado para itens com natureza de consumo intermitente. Esta dificuldade pode resultar em falhas nos processos de aquisição e, em consequência, provocar problemas como a ruptura de estoque, ou o desperdício de materiais em excesso. Discrepâncias no processo de aquisição de produtos podem ocorrer na área hospitalar, de varejo, de vendas, de manutenção, e outros segmentos distintos como o automotivo, aeronáutico, marítimo e de mineração. Sendo este último a delimitação desta pesquisa.

As peças de reposição, são exemplos de itens com demandas normalmente classificadas como esporádica ou intermitente, formando séries temporais diferentes das convencionais, devido à presença de vários períodos com demanda zero. Em muitos casos, esses itens podem representar até $60 \%$ do valor total do estoque (JOHNSTON; BOYLAN; SHALE, 2003). Quanto maior o volume, preço unitário, criticidade e risco de obsolescência destes itens, maior será o possível impacto dos desvios de predição da demanda.

Não é simples predizer a demanda intermitente devido à sua natureza errática e as vezes irregular (TEUNTER; SANI, 2009). Os intervalos de demanda são pouco frequentes, contendo muitos períodos com demanda zero, juntamente com tamanhos variados.

Vários métodos para predição de demanda são discutidos na literatura. A primeira sugestão de que os métodos tradicionais de predição, como a Média Móvel Simples (MMS) e Suavização Exponencial Simples (SES) podem ser inadequados 
para itens de baixo ou baixíssimo giro, surgiu em 1972 com Croston. Ele demonstrou que estes métodos podem levar a decisões de estoque inadequadas e propôs um procedimento alternativo de predição, em que o intervalo entre demandas e o tamanho da demanda são atualizados separadamente, e somente em períodos com demanda positiva. Neste método, a mesma constante de suavização pode ser utilizada para as duas séries decompostas e o estimador consiste na divisão do tamanho da demanda pelo intervalo entre demandas então suavizados, visando obter predições melhores.

A abordagem de Croston (1972) é utilizada para predizer demanda intermitente, inclusive quando estão envolvidas grandes quantidades de itens, e está presente em programas computacionais como Forecast Pro, SAS e SAP/APO. Todavia, foi demonstrado um viés neste método por Syntetos e Boylan (2001) e outras opções foram propostas por Syntetos, Boylan (2005), Shale, Boylan e Johnston (2006) e Teunter, Syntetos e Babai (2011).

Apesar do desenvolvimento ao longo dos anos, muitas indústrias continuam utilizando abordagens tradicionais de predição, como Média Móvel Simples (MMS) e Suavização Exponencial Simples (SES). Embora existam modelos de classificação para apoiar uma escolha entre alguns métodos alternativos, a literatura não oferece diretrizes universais para selecionar o método mais adequado para séries temporais intermitentes (SYNTETOS; BOYLAN, 2005).

Com base no cenário de estudos aqui apresentado, o objetivo principal deste trabalho foi otimizar e analisar o processo de predição de demanda de peças de reposição com ênfase na demanda intermitente. Os modelos de predição foram testados e validados utilizando dados reais de demanda de peças de reposição de uma empresa do setor de mineração. 


\section{OBJETIVOS}

\subsection{Geral}

Comparar o desempenho dos métodos para predição de demanda, especialmente demanda intermitente, utilizando dados de demanda real e previamente analisados, por meio de várias abordagens.

\subsection{Específicos}

a) Realizar a predição de demandas de peças de reposição do setor de mineração por meio das abordagens de (i) Croston (1972); (ii) SBA (SYNTETOS; BOYLAN, 2005); (iii) SBJ (SHALE; BOYLAN; JOHNSTON, 2006); (iv) TSB (TEUNTER; SYNTETOS; BABAI, 2011);

b) Comparar os resultados obtidos utilizando esses métodos com os dados obtidos com Média Móvel Simples (MMS) e Suavização Exponencial Simples (SES), além das referências NAÏVE e ZERO;

c) Propor a otimização do processo de predição a partir de cada medida de erro especificada. 


\section{REVISÃO DE LITERATURA}

O propósito deste estudo é a contextualização do processo de predição de demanda intermitente e formas de se obter um melhor desempenho. Pretende-se contribuir para o melhor entendimento de alguns modelos aplicáveis e os possíveis benefícios de sua utilização.

\subsection{Predição da demanda intermitente}

Os estudos de predição de demanda intermitente começaram com a indústria da aviação, a fim de predizer o consumo de peças de reposição, uma vez que são de custo elevado, com intervalos de consumo aleatórios e possuem dificuldades de manuseio e movimentação.

A Suavização Exponencial Simples (SES) foi o primeiro método de predição aplicado a dados de natureza intermitente. O modelo consiste na atribuição de um fator de ponderação para cada valor da série temporal, de forma que os valores de demanda mais recentes recebem ponderações maiores (PELLEGRINI; FOGLIATTO, 2001).

Apesar de ser um método de predição considerado simples, é possível observar a utilização do método de SES em muitos trabalhos de pesquisa e em programas computacionais de predição utilizados na indústria. Este método apresenta um comportamento interessante, quando comparado com os métodos CRO (CROSTON, 1972) e SBA (SYNTETOS; BOYLAN, 2005) que são considerados de maior complexidade (KOURENTZES, 2014; WALLSTRÖM; SEGERSTEDT, 2010).

No entanto, a utilização da Suavização Exponencial Simples (SES) para predições de demandas intermitentes, quase sempre, produzem níveis inadequados de estoque, porque a reposição de quantidades constantes em intervalos fixos pode gerar níveis de estoque de até o dobro da quantidade realmente necessária (CROSTON, 1972).

Croston (1972) concluiu que métodos tradicionais, incluindo o SES, não tinham um bom desempenho em séries temporais intermitentes. Ele então desenvolveu outro método para superar essas dificuldades, utilizando estimativas separadas do tamanho da demanda e da frequência em que a demanda irá ocorrer, 
aplicando-se uma mesma constante de suavização $(\alpha)$. A predição final é então determinada pelo quociente das duas estimativas e representa a procura média por período. Quando a demanda ocorre em todos os períodos de revisão, tornando-se uma série contínua, o método de Croston passa a ser idêntico ao método de Suavização Exponencial Simples (SES).

A maioria dos trabalhos sobre predição de demanda intermitente faz referência ao artigo de Croston (1972). Este artigo seminal introduziu a ideia de separar tamanhos de demanda e intervalos de tempo para obtenção de predições melhores do que a tradicional Suavização Exponencial Simples (SES). De acordo com Croston (1972), esse modelo tem uma vantagem considerável de operar tanto para demandas intermitentes quanto para demandas frequentes, ou seja, o sistema se comporta da maneira padrão se uma demanda ocorrer ou não a cada intervalo de predição. O sistema proposto evita os erros inerentes, quando a suavização exponencial for aplicada a demandas intermitentes. Os níveis de estoque em excesso seriam evitados. O método é amplamente utilizado na indústria e incorporado em vários sistemas computacionais de predição (SYNTETOS; BOYLAN; CROSTON, 2005).

O método foi reivindicado para ser imparcial mas, apesar de sua superioridade teórica, os benefícios registrados na literatura foram modestos quando comparados com técnicas de predição mais simples, como a Suavização Exponencial Simples (SES) (WILLEMAIN et al., 1994).

Alguns autores como Sani e Kingsman (1997) sugeriram a ocorrência de perdas no desempenho do método de Croston. Foi demonstrado que o método possuía viés e, desde então, algumas modificações foram apresentadas na literatura, como em Syntetos e Boylan (2001). Os autores afirmaram que o método de Croston (1972) é, teoricamente, superior à suavização exponencial e pode fornecer benefícios tangíveis aos fabricantes na predição de demanda intermitente. Porém, relataram que os resultados práticos obtidos nas indústrias nem sempre correspondiam satisfatoriamente, em comparação com os resultados das simulações. Quando o modelo de Croston (1972) foi utilizado com dados reais, observou-se que métodos de predição mais simples pareciam fornecer resultados mais precisos e levar a um controle de estoque mais eficaz. Observaram em todas estas execuções de simulação que a tendência da constante de suavização era ter 
valores acima de 0,15 e concluíram que o método de Croston (1972) seria recomendado, portanto, apenas para valores baixos da constante de suavização $(\alpha)$.

Vale ressaltar o longo período, aproximadamente, 30 anos entre a publicação do método de Croston (1972) e a comprovação do viés por Syntetos e Boylan (2001). Avanços na implementação de banco de dados nas empresas, e a busca por melhores margens por meio da otimização de inventário, podem ter contribuído para o aumento de pesquisas nesta área nas duas últimas décadas.

Posteriormente foi desenvolvido o método de Aproximação de Syntetos e Boylan - Syntetos Boylan Approximation (SBA) que trouxe melhoria na proposta de Croston (1972), como confirmado em outros estudos destes mesmos autores (SYNTETOS; BOYLAN; CROSTON, 2005).

Por meio da análise de mais de 3.000 produtos do setor automotivo de demanda intermitente, Syntetos e Boylan (2005) demonstraram que o método SBA é mais acurado que o de Croston (1972). Outra comparação realizada por Syntetos e Boylan (2006) mostrou, novamente, um desempenho superior do modelo SBA, seguido pelo método da Média Móvel e de Croston (1972).

Neste método, que foi uma adaptação do método de Croston, foi inserido um corretor de viés na equação de predição. No entanto, estudos posteriores realizados por Teunter e Sani (2009) e Wallström e Segerstedt (2010) mostraram que, apesar desta modificação, ainda pode surgir viés quando a intermitência da série temporal em análise é baixa.

Uma modificação diferente, mais apropriada, denominada de modelo SBJ, foi então apresentada, considerando que os pedidos de itens de reposição chegam como um processo de Poisson (SHALE; BOYLAN; JOHNSTON, 2006).

O método SBJ não foi muito citado na literatura de predição de demanda intermitente ou poucas aplicações foram apresentadas. Syntetos e Boylan (2010), por exemplo, analisaram os procedimentos mais citados de estimação de demanda intermitente, com relação à variação de suas estimativas, mas não mencionaram o método SBJ. No entanto, Kourentzes (2014) incluiu este método em sua pesquisa comparativa.

Um novo procedimento de predição, denominado de TSB, que tratava da questão da obsolescência do inventário, foi apresentado em 2011 por Teunter, Syntetos e Babai (2011). Neste método, a predição é ajustada para baixo toda vez que for encontrada uma demanda zero, diferente de Croston (1972), que considera 
que a predição fica constante depois da demanda zero. Como resultado, o novo método foi considerado mais adequado para lidar com problemas de obsolescência. Este método também foi considerado estatisticamente imparcial para todos os pontos no tempo. O seu desempenho foi avaliado por meio de um estudo de simulação sobre dados gerados e apresentou resultados comparáveis aos obtidos com os outros métodos discutidos na literatura.

O método possui a característica de atualizar o valor da estimação de probabilidade de procura continuamente ao longo de diversos períodos. Isto permite modelar uma diminuição gradual de demanda, a ponto da demanda se tornar zero, para itens descontinuados. Se essa obsolescência não for modelada, será formado um excesso de estoque para estes itens (TEUNTER; SYNTETOS; BABAI, 2011).

O método TSB utiliza estimativas separadas e exponencialmente ajustadas do tamanho da demanda e da probabilidade de demanda. Cada etapa de suavização utiliza um parâmetro diferente. Teunter, Syntetos e Babai (2011) argumentam que o parâmetro de suavização da probabilidade de demanda deve ser menor que o parâmetro de suavização do tamanho da demanda. Estes autores relatam bom desempenho do método, mas alertam para a importância de escolher cuidadosamente os dois parâmetros. Como o método permite que o valor da predição se aproxime de zero em períodos contínuos sem demanda, isto o torna diferenciado em relação aos demais métodos, pois permite tomar decisões de gestão de estoques. Tornou-se, portanto, uma alternativa para a questão da obsolescência, que não tinha sido estudada até o momento.

O método ingênuo (Naïve) é um caso especial do método TSB e do método SES, quando o valor da (s) constante (s) de suavização é igual a 1 . O método ingênuo, apesar de sua simplicidade, é muito utilizado e apresenta um desempenho interessante em predições de séries temporais financeiras e econômicas (HYNDMAN; ATHANASAUPOLOS, 2018).

O método ingênuo é utilizado como método de referência (benchmarking) e considera que as predições futuras são iguais ao último valor observado. Já o método zero estima, para cada horizonte, uma predição de valor zero, e também é utilizado como referência.

A média móvel é uma técnica de predição que altera a influência dos valores passados de uma série na projeção futura. Consiste no cálculo da média dos valores 
mais recentes, em que, a cada período, o valor mais antigo é substituído pelo mais recente, assim modificando a média (MORETTIN; TOLOI, 2006).

O detalhamento das equações dos métodos está descrito na Seção 1.8.

\subsubsection{Classificação de demanda}

Syntetos e Boylan (2005) analisaram uma gama mais ampla de padrões intermitentes de demanda e apresentaram uma classificação para orientar a seleção dos métodos de predição. Estes autores concluíram que há categorias de demanda mais adequadas ao método original de Croston (1972) e há outras que funcionam melhor com a modificação de Syntetos e Boylan (2005).

Os itens de reposição foram classificados por Syntetos e Boylan (2005) em quatro quadrantes, levando-se em consideração dois eixos que se cruzam perpendicularmente. Um dos eixos é o intervalo médio entre demandas $(p)$ e o outro é quadrado do coeficiente de variação dos tamanhos de demanda $\left(c_{v}^{2}\right)$. Os pontos de divisão nos eixos ( $p=1,32$ e $\left.c_{v}^{2}=0,49\right)$ foram estabelecidos teoricamente e testados com 3.000 séries de demanda de autopeças. Foram comparados os métodos de Suavização Exponencial Simples (SES), Croston (CRO) e Aproximação de Syntetos e Boylan (SBA). Neste estudo, o método de Croston foi indicado para o quadrante de itens "Suave" $\left(p<1,32\right.$ e $\left.c_{v}^{2}<0,49\right)$ e o método SBA para os demais quadrantes. A demanda intermitente foi categorizada nos quadrantes Esporádico e Baixo Giro, conforme ilustrado na Figura 1.

Figura 1- Classificação de demanda segundo Syntetos e Boylan (2005).

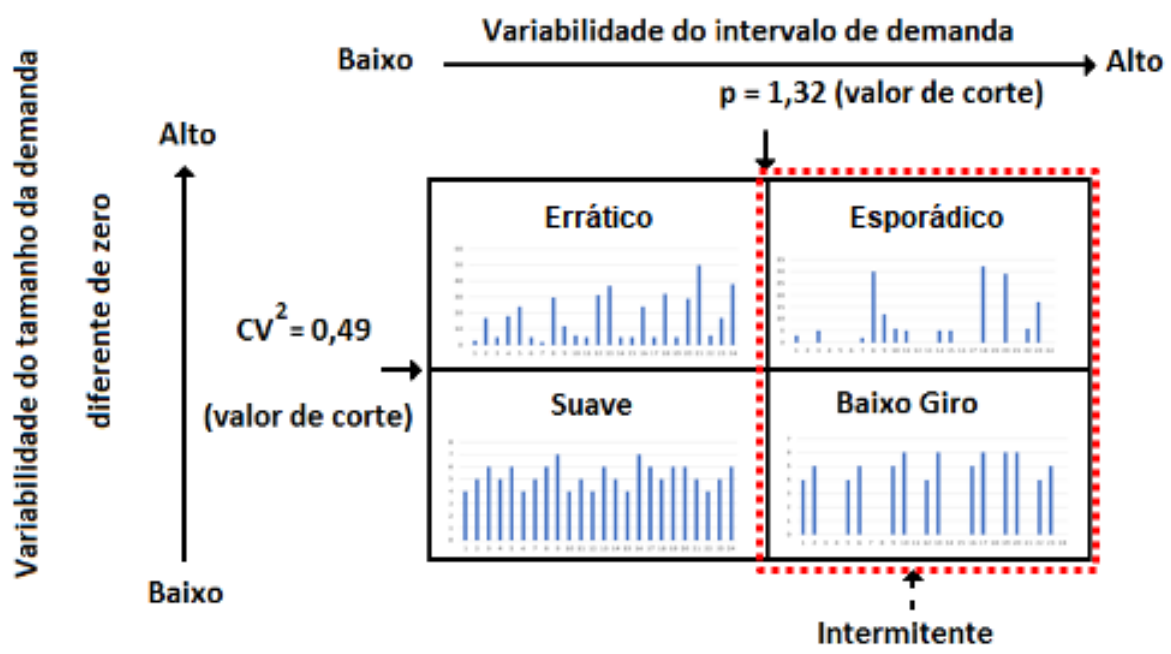

Fonte: SYNTETOS;BOYLAN., 2005. (Adaptado pelo autor). 
O detalhamento das equações para categorização de demanda está descrito na Seção 1.7.3.

\subsection{Medidas de acurácia}

Para comparar o desempenho de cada método de predição de demanda, um processo típico é a utilização de medidas de erros ou de acurácia operacional. Estas medidas podem ser utilizadas também como funções de perda no processo de otimização das predições (KOURENTZES, 2014).

Uma medida comum para controle de estoque é o Desvio Médio Absoluto (DMA), que consiste no módulo da diferença entre o valor real e o estimado (TUBINO, 2000):

$$
\bar{D}_{a b s}=\frac{1}{n} \sum_{i=1}^{n} y_{i}-\hat{y}_{i}
$$

Em que:

$\bar{D}_{a b s}=$ Desvio médio absoluto (DMA);

$y_{i}=$ Valor observado (ou real);

$\hat{y}_{i}=$ Valor predito (ou estimado);

$n=$ Número de observações.

Considerando a otimização de séries temporais convencionais, uma outra função de perda popular é o Desvio Quadrático Médio (DQM):

$$
\bar{D}_{\text {quad }}=\frac{1}{n} \sum_{i=1}^{n}\left(y_{i}-\hat{y}_{i}\right)^{2}
$$

Em que:

$$
\begin{array}{ll}
\bar{D}_{\text {quad }} & =\text { Desvio quadrático médio (DQM); } \\
y_{i} & =\text { Valor observado (ou real); } \\
\hat{y}_{i} & =\text { Valor predito (ou estimado); } \\
n & =\text { Número de observações. }
\end{array}
$$


Uma proposta diferente para determinar o desempenho dos itens com demanda intermitente é dada por Hyndman et al., (2006), denominado Erro Médio Absoluto Escalonado (EMAE) que, segundo o autor, é uma medida de erro mais adequada, pois nunca se obtém valores infinitos ou indefinidos. O cálculo do EMAE é realizado pela divisão do erro da predição que está sendo utilizada, pela média do erro do método ingênuo (NAÏVE). No modelo de predição do método ingênuo, a predição da demanda do próximo período é igual à demanda do período atual, ou seja, um período à frente. O valor do EMAE é calculado usando as Equações (3) e (4), em que $D_{E}$ é o erro escalonado:

$$
\begin{gathered}
D_{E}=\frac{D_{p}}{\left[\left(\frac{1}{n-1}\right) \cdot \sum_{i=2}^{n}\left(\left|y_{i}-y_{i-1}\right|\right)\right]} \\
\bar{E}_{a b s E}=\frac{1}{n} \sum_{i=1}^{n}\left|D_{E}\right|
\end{gathered}
$$

Em que:

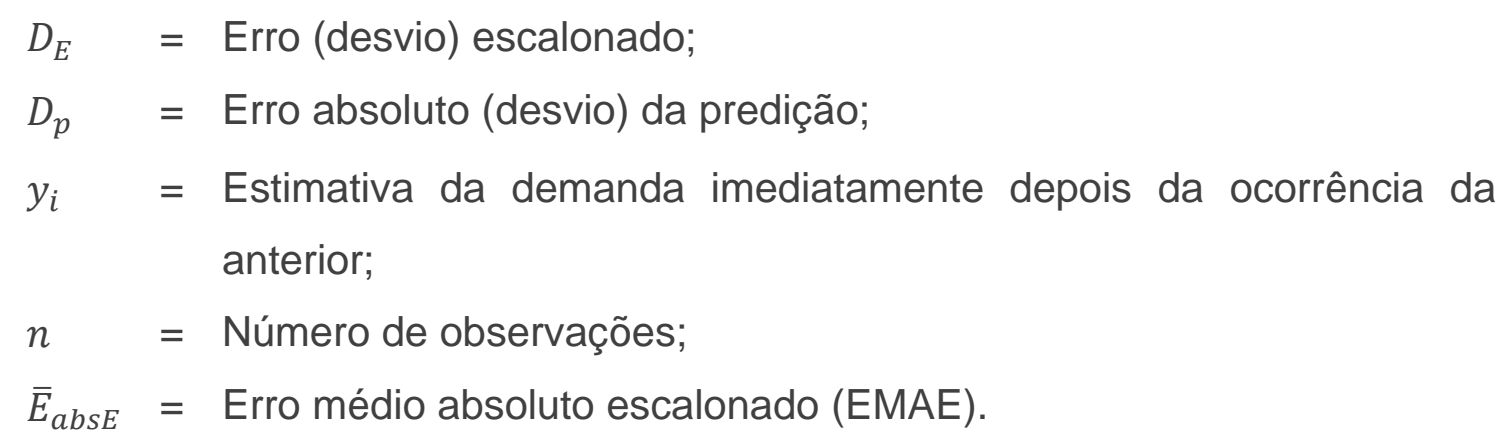

Estas são, portanto, algumas das medidas consideradas convencionais mais utilizadas: o Desvio Médio Absoluto (DMA), o Desvio Quadrático Médio (DQM) e o Erro Médio Absoluto Escalonado (EMAE).

A aplicabilidade dessas medidas de erro para séries temporais intermitentes, no entanto, tem sido contestada por alguns autores. Estas medidas tendem a polarizar as predições em favor da predição de demanda zero e não forneceriam boa aproximação para as decisões de inventário. Outras medidas para avaliação de desempenho foram sugeridas como: Períodos em Estoque (PEE), Período em Estoque Escalonado Absoluto Modificado (PEEEAm), Taxa do Quadrado da Média (TQM) e Taxa Absoluta Média (TAM). Estas são medidas de somatório acumulativo, 
que visam impedir a preferência pelas predições de demanda zero em uma série temporal muito intermitente (KOURENTZES, 2014).

Wällstrom e Segerstedt (2010) propuseram uma metodologia para determinar períodos em estoque (PEE), que consiste em se obter o número total de períodos em que uma unidade do item foi predita para estar dentro ou fora do estoque. Esta medida assume um estoque "fictício", em que o excesso ou a falta de peças é determinado, calculando o período em estoque usando a Equação (5):

$$
P_{E E}=-\sum_{i=1}^{n} \sum_{j=1}^{i}\left(y_{j}-\hat{y}_{j}\right)
$$

Em que:

$$
\begin{aligned}
& P_{E E}=\text { Períodos em estoque (PEE); } \\
& y_{j} \quad=\text { Valor observado (ou real); } \\
& \hat{y}_{j} \quad=\text { Valor predito (ou estimado); } \\
& n \quad=\quad \text { Número de observações. }
\end{aligned}
$$

Embora essa metodologia não seja imune em preferir as predições de demanda zero para uma série temporal muito intermitente, é mais robusta do que as medidas de erro convencionais, devido ao seu somatório acumulativo (KOURENTZES, 2014).

A medida PEE foi também considerada uma métrica de viés e não medida de erro, sendo aplicável como combinação a outras medidas (KOURENTZES, 2014; WALLSTRÖM; SEGERSTEDT, 2010).

O PEE originalmente proposto é uma medida dependente de escala. Outra opção para se agregar uma série e obter um erro independente de escala, é a divisão do módulo do período em estoque pela demanda média da amostra de cada item. O resultado é a medida do Período em Estoque Escalonado Absoluto Modificado (PEEEAm) para o período da predição e pode ser calculado usando a Equação (6):

$$
P_{\text {EEEAm }}=\frac{\left|-\sum_{i=1}^{h} \sum_{j=1}^{i}\left(y_{j}-\hat{y}_{j}\right)\right|}{\frac{1}{n} \sum_{i=1}^{n} y_{i}}
$$


Em que:

$$
\begin{array}{ll}
P_{\text {EEEAm }} & =\text { Período em estoque escalonado absoluto modificado; } \\
y_{j} & =\text { Demanda observada (ou real) na amostra teste; } \\
\hat{y}_{j} & =\text { Valor predito (ou estimado); } \\
h & =\text { Período de predição; } \\
y_{i} & =\text { Valor observado (ou real); } \\
n & =\text { Número de observações. }
\end{array}
$$

Uma vantagem do Período em Estoque Escalonado Absoluto Modificado (PEEEAm) é penalizar adequadamente as predições de demanda zero (KOURENTZES, 2014). O autor também salienta que o método de Croston e suas variantes fornecem uma "taxa de demanda" com unidade diferente da demanda real, e medir esta diferença não seria significativo. O DMA e o DQM seriam ineficientes neste aspecto. Embora o PEE supere isso, devido à sua base de erros acumulativos, essa propriedade das predições poderia ser utilizada para derivar novas metodologias para otimização através de uma taxa acumulativa do erro $\left(r_{i}\right)$ :

$$
r_{i}=\hat{y}_{i}-\frac{1}{n} \cdot \sum_{j=1}^{n} y_{j}
$$

Em que:

$$
\begin{aligned}
r_{i} & =\text { Taxa acumulativa do erro; } \\
\hat{y}_{i} & =\text { Valor predito (ou estimado) } \\
y_{j} & =\text { Valor observado (ou real); } \\
n & =\text { Número de observações. }
\end{aligned}
$$

A partir de $r_{i}$ é possível construir outras duas versões, denominadas de Taxa Média Absoluta (TAM) e Taxa do Quadrado da Média (TQM). O detalhe envolvendo a TAM e a TQM é ajustar as predições de demanda intermitente pela demanda média por período, ao invés da demanda realizada (KOURENTZES, 2014), Equações (8) e (9): 


$$
\begin{gathered}
\bar{T}_{a b s}=\sum_{i=1}^{n}\left|r_{i}\right| \\
\bar{T}_{\text {quad }}=\sum_{i=1}^{n} r_{i}^{2}
\end{gathered}
$$

Em que:

$\bar{T}_{a b s}=$ Taxa média absoluta (TAM);

$r_{i} \quad=$ Taxa acumulativa do erro;

$n=$ Número de observações;

$\bar{T}_{\text {quad }}=$ Taxa do quadrado da média (TQM).

Para estas medidas de erro, o momento da demanda diferente de zero não afeta os erros, enquanto a probabilidade e o tamanho da demanda são considerados. Estas duas medidas são opções às medidas de erros tradicionais, utilizadas como função de perda para otimizar os métodos de predição de séries com demanda intermitente (KOURENTZES, 2014).

Para reforçar o conceito de "taxa de demanda", descreve-se o exemplo a seguir, adaptado do conceito de Kourentzes (2014):

1. Supondo que a demanda não nula predita de um item seja 3 unidades e que o intervalo entre demanda preestabelecido seja de 6 meses. A predição de Croston, por exemplo, seria de 0,5 unidades/mês. Em vez de produzir uma predição de demanda e intervalo, obtém-se uma taxa de demanda que combina esses dois fatores. A interpretação em Kourentzes (2014) para esta predição, é que haverá uma necessidade de 0,5 unidades extras a cada período. Neste caso seria observada uma unidade completa de demanda em 2 meses, mas com tempo desconhecido.

2. Para um horizonte de predição de 8 meses, por exemplo, a demanda seria de 4 unidades, mas novamente com tempo indeterminado. Essas unidades poderiam estar distribuídas em dois meses, três, quatro ou em todos os meses. Também poderiam estar concentradas apenas no primeiro mês ou no oitavo mês. Este tempo é difícil de predizer, e, portanto, a demanda é distribuída ao longo dos períodos de intervalo esperados. 
3. Esta interpretação para as taxas TAM e TQM é diferente de medidas de erro como DMA e DQM, que calculam a diferença pontual entre a predição e a demanda real observada. A proposta de Kourentzes (2014) é que a interpretação da taxa, ao ser traduzida em uma decisão de inventário, garanta um estoque suficiente e superior ao da utilização de medidas convencionais para a demanda intermitente.

Para as medidas DMA, DQM, PEE, TAM e TQM também é possível uma versão escalonada, dividindo-se o valor da medida de erro pela média da amostra. $\bigcirc$ erro escalonado é um erro relativo, cujo objetivo é a remoção da escala, para que os erros de diferentes séries temporais que compõem o conjunto de dados, possam ser comparados. As medidas EMAE e PEEEAm já contemplam o escalonamento em suas equações.

\subsection{Otimização das predições}

Para ilustrar o processo de otimização de parâmetros a partir das funções de perda dos erros das predições, seguirá como exemplo o modelo da Suavização Exponencial Simples (SES), que constitui base para outros modelos. A busca será pelos parâmetros $\alpha$ (constante de suavização) e $l_{0}$ (predição inicial em $t=0$ ).

\subsubsection{Otimização da suavização exponencial}

No método SES, quanto menor a constante de suavização (parâmetro $\alpha$ ), mais suavizada fica a curva e menor o efeito de grande variação na demanda. Quanto maior o valor da constante de suavização $(\alpha)$, menor o tempo de reação da série a mudanças bruscas na demanda. Desta forma, antes de implementar um modelo, diferentes valores da constante $(\alpha)$ devem ser testados e ajustados de acordo com as medidas de erro da predição e tipo de série temporal. Uma opção para se encontrar os parâmetros é a otimização utilizando a função de perda da amostra (KOURENTZES, 2014).

Existem várias formas para obtenção dos parâmetros $\alpha$ (constante de suavização) e $l_{0}$ (predição inicial em $\mathrm{t}=0$ ) da Suavização Exponencial Simples (SES). Para o parâmetro $\alpha$ (constante de suavização), pode-se arbitrar um valor por experiência, utilizar a técnica de enumeração simples, ou utilizar métodos de otimização que permitam obter valores adequados para a função de perda do 
conjunto da amostra. Alguns trabalhos, como Croston (1972), Syntetos e Boylan (2005) e Teunter e Sani (2009) aconselham que este parâmetro de suavização tenha valores nos limites entre 0,05 e 0,3, por exemplo.

No modelo de enumeração simples, depois de calcular os valores de erro de predição para toda a sequência, o valor da constante de suavização é alterado em um pequeno incremento, e um novo cálculo é realizado. Este procedimento é repetido até que todos os valores possíveis de parâmetros sejam enumerados. Seleciona-se o valor de $\alpha$ que resultou no menor valor de erro. Contudo, trabalhos como de Petropoulos et al., (2013), relatam que a seleção de parâmetros otimizados permite obter resultados melhores e diminui a tendenciosidade dos métodos, quando comparados com os mesmos métodos com valores arbitrados.

Para o segundo parâmetro da suavização exponencial, a predição inicial em $\mathrm{t}=0\left(l_{0}\right)$ também existem várias recomendações na literatura para sua escolha. Por exemplo, o valor inicial $\left(l_{0}\right)$ pode ser definido como o primeiro elemento de uma sequência $\left(l_{0}=y_{1}\right)$, como a média da série observada ou a média de três, quatro ou mais elementos iniciais da sequência, com o objetivo de suavizar discrepâncias aleatórias. Porém, nenhum destes métodos pode garantir a ausência de erros perceptíveis nos primeiros estágios do algoritmo de suavização. Desta forma, para minimizar o impacto dos problemas associados com a escolha dos valores iniciais (principalmente para sequências curtas), utiliza-se o método de otimização para definir $l_{0}$ (valor inicial), que resultará no erro de predição mínimo.

Kourentzes (2014) demonstrou que a eficiência dos métodos, quando se recorre à utilização de parâmetros de suavização e valores iniciais otimizados, resultam em melhorias consideráveis na aplicação do modelo de Croston e seus derivados. $O$ processo de otimização consiste em minimizar a função de perda do erro, para se obter os valores ótimos dos parâmetros do modelo. Para melhor entendimento deste processo, segue o desenvolvimento algébrico de Sanjoy (2011), para o método de SES.

Equação de predição para Suavização Exponencial Simples (SES):

$$
\hat{y}_{t+h \mid t}=l_{t}
$$

Equação de Suavização (Seção 1.8.4): 


$$
l_{t}=\alpha y_{t}+(1-\alpha) l_{t-1}
$$

A otimização por meio de seleção dos parâmetros ótimos de inicialização, implica em determinar os parâmetros $\alpha$ e $l_{0}$, em que $l_{0}$ é um valor inicial a considerar para o período zero e $\alpha$ é o parâmetro de suavização. Então, segue que:

$$
\begin{gathered}
l_{1}=\alpha \cdot y_{1}+(1-\alpha) l_{0} \\
l_{2}=\alpha \cdot y_{2}+(1-\alpha) l_{1}=\alpha \cdot y_{2}+\alpha(1-\alpha) y_{1}+(1-\alpha)^{2} l_{0} \\
l_{3}=\alpha y_{3}+(1-\alpha) l_{2}=\sum_{j=0}^{2} \alpha(1-\alpha)^{j} y_{3-j}+(1-\alpha)^{3} l_{0} \\
l_{t}=\sum_{j=1}^{t-1} \alpha(1-\alpha)^{j} y_{t-j}+(1-\alpha)^{t} l_{0}
\end{gathered}
$$

Então, o modelo de predição resulta na Equação (16):

$$
\hat{y}_{t+h \mid t}=\sum_{j=1}^{t} \alpha(1-\alpha)^{t-j} y_{j}+(1-\alpha)^{t} l_{0}, \quad(0 \leq \alpha \geq 1)
$$

Em que:

$$
\begin{aligned}
\hat{y}_{t+h \mid t} & =\text { Predição para o período posterior a } t \\
y_{j} & =\text { Demanda observada; } \\
l_{0} & =\text { Valor inicial em } t=0 ; \\
\alpha & \left.=\text { Declividade da curva } y_{(t+1)}\right)(\text { constante de suavização entre } 0 \text { e } 1) .
\end{aligned}
$$

Para a etapa de minimização do erro, uma medida muito comum é o Desvio Quadrático Médio (DQM), no entanto, outras medidas podem ser utilizadas, como as descritas na Seção 1.4.

O DQM utiliza exponenciação e, consequentemente, possui boas propriedades matemáticas que facilitam o cálculo de sua derivada em comparação 
com o Desvio Médio Absoluto $\left(\bar{D}_{a b s}\right)$. Este é um requisito importante quando se utiliza um modelo de algoritmo com Gradiente Descendente. Porém, este benefício não é igualmente percebido em equações não-lineares, como as apresentadas a seguir:

$$
\begin{gathered}
D_{a b s}=y_{t}-\hat{y}_{t \mid t-1} \\
\bar{D}_{\text {quad }}=\frac{1}{T} \sum_{t=1}^{T}\left(y_{t}-\hat{y}_{t \mid t-1}\right)^{2}=\frac{1}{T} \sum_{t=1}^{T}\left(D_{a b s}\right)^{2}
\end{gathered}
$$

Em que:

$D_{a b s}=$ Desvio absoluto entre predição e demanda observada;

$\bar{D}_{\text {quad }}=$ Desvio quadrático médio;

$\hat{y}_{t \mid t-1}=$ Predição para o tempo $t$;

$y_{t} \quad=$ Demanda observada;

$T=$ Tempo total.

Combinando-se as Equações (16) e (18), em função de $\alpha$ e de $l_{0}$, tem-se:

$$
\bar{D}_{\text {quad }}=\frac{1}{T} \sum_{t=1}^{T}\left[\left(y_{t}-\sum_{j=1}^{t} \alpha(1-\alpha)^{t-j} y_{j}+(1-\alpha)^{t} l_{0}\right)\right]^{2}
$$

Minimizando a soma dos quadrados destes erros ao longo de toda a amostra, pode-se determinar o valor ideal do coeficiente $\alpha$ e o valor inicial $l_{0}$ para uma dada sequência.

No entanto, para uma procura automática para vários itens, séries longas e com mais parâmetros de otimização, será necessário um grande esforço computacional e a solução numérica por algoritmos passa a ser uma melhor opção. Em alguns algoritmos de pacotes de predição estima-se os parâmetros por meio da máxima verossimilhança. Para a Suavização Exponencial Simples, por exemplo, a abordagem numérica e por máxima verossimilhança são equivalentes.

Um exemplo para um item específico está ilustrado no gráfico da Figura 2, com comparação da soma dos quadrados de erros de predição usando um passo, 
em função do coeficiente $\alpha$ e, no gráfico da Figura 3 , em função de $l_{0}$, para uma sequência de teste.

Outros métodos não lineares são disponíveis para uma função multivariável, como Mínimo Quadrados; Descida de Gradiente; Método de Gauss-Newton; Método de Levenberg-Marquardt; Método de Powell e Método Quasi-Newton. Como escolha para ilustração deste trabalho, será descrito o Método de Powell. O método de Powell não requer cálculo de derivativos da função objetivo e pode demonstrar uma abordagem para otimização da predição.

Figura 2- Exemplo de busca de $\alpha$ para uma Suavização Exponencial Simples (SES) com $l_{0}$ definido.

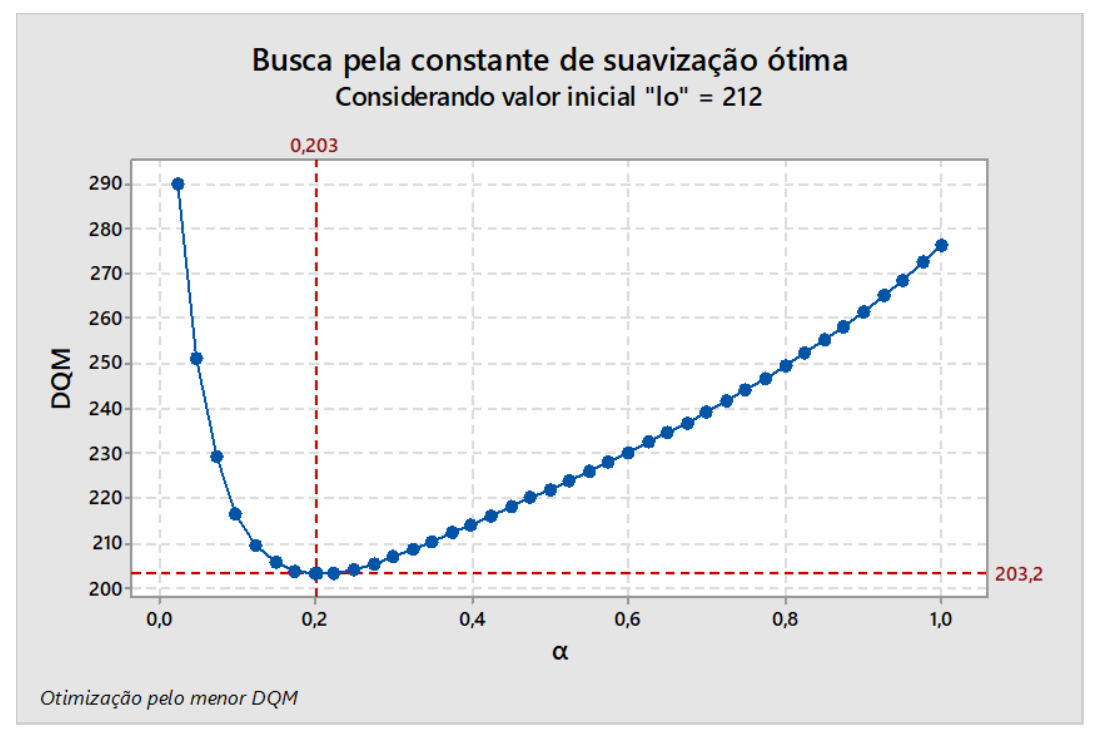

Fonte: O autor, 2020.

Figura 3- Exemplo busca de $l_{0}$ ótimo para uma Suavização Exponencial Simples (SES) $\operatorname{com} \alpha$ definido.

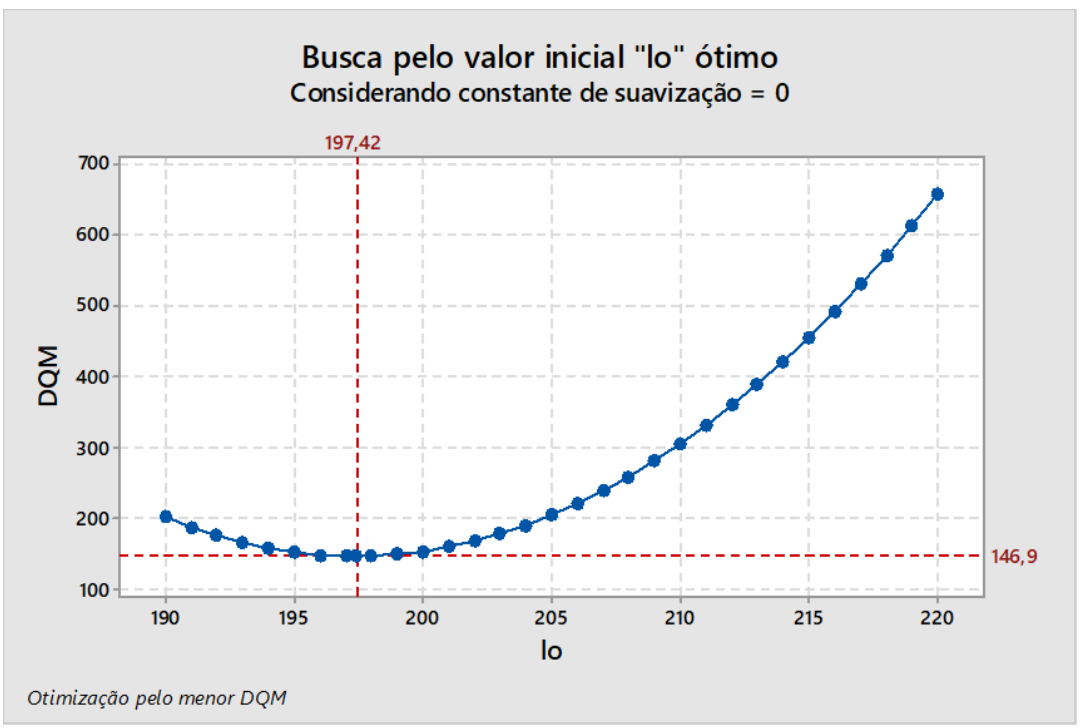

Fonte: O autor, 2020. 


\subsubsection{Método das Direções Conjugadas de Powell}

O método de Powell considera que toda função pode ser aproximada por uma função quadrática próxima do mínimo. Nesse método, a cada passo a função a ser minimizada é aproximada localmente por uma função quadrática (SILVA, 2003):

$$
f(x)=\frac{1}{2} \boldsymbol{x}^{T} \cdot \boldsymbol{Q} \cdot \boldsymbol{x}+\boldsymbol{b}^{T} \cdot \boldsymbol{x}+\boldsymbol{c}
$$

Em que:

$$
\begin{aligned}
& f=\text { Função objetivo a ser minimizada; } \\
& \boldsymbol{x}=\text { Vetor de variáveis independentes; } \\
& \boldsymbol{Q}=\text { Matriz constante de dimensões compatíveis ao problema; } \\
& \boldsymbol{b}=\text { Grandeza vetorial; } \\
& \boldsymbol{c}=\text { Grandeza escalar. }
\end{aligned}
$$

Seja um conjunto de direções, $s_{i}, i=1,2 \ldots$ de $Q$ direções conjugadas linearmente independentes, definido pela Equação (21):

$$
s_{i}^{T} \boldsymbol{Q} \cdot \boldsymbol{s}_{j}=0 \text { para } i \neq j
$$

Em que:

$\boldsymbol{Q}=$ Matriz constante de dimensões compatíveis ao problema;

$\boldsymbol{s}=$ Direção conjugada.

Considerando as premissas definidas pela Equação (21), pode ser mostrado que, se $f$ for minimizada ao longo de cada direção $s$ definida, então o mínimo de $f$ será encontrado no (ou antes do) $n$-ésimo passo, independentemente do ponto inicial, dado que erros de arredondamento não sejam acumulados, em $n$ é o número de variáveis. É importante que as direções sejam linearmente independentes, como definido na Equação (21), caso contrário não há convergência para o mínimo.

Powell sugere um método conveniente de gerar $Q$ direções conjugadas linearmente independentes. No entanto, às vezes esse método pode gerar direções linearmente dependentes quando, nesse caso, não converge para o mínimo. 
A estratégia de Powell é baseada na seguinte propriedade: se $x_{1}$ e $x_{2}$ são dois pontos e $S$ é uma direção especificada e, se $x_{1 s}$ corresponde ao ponto mínimo de $f$ na linha paralela a $s$ iniciando em $x_{1}$, e se $x_{2 s}$ é o ponto mínimo de $f$ na linha paralela a $S$ iniciando em $\boldsymbol{x}_{2}$, então $S$ e a direção $\left(x_{2 s}-x_{1 s}\right)$ serão $Q$ direções conjugadas (SILVA, 2003).

O algoritmo de Powell é descrito pelos passos a seguir (POWELL1, 1964, citado por SILVA, 2003):

1) Minimize $f$ ao longo das direções coordenadas (busca variada), iniciando em $x_{0}^{k}$ e gerando os pontos $x_{1}^{k}, \cdots, x_{n}^{k}$ em que $k$ é o número do ciclo.

2) Depois de encerrar a busca univariada, encontrar 0 índice $m$ correspondente à direção em que a função $f$ apresenta o maior decréscimo indo de $x_{m-1}^{k}$ para $x_{m}^{k}$.

3) Calcular a direção "padrão" (soma de todos os movimentos univariados) e determinar o valor de $\alpha$ que minimize $f$ tal que:

$$
x=x_{0}^{k}+\alpha \cdot s_{p}^{k}
$$

4) Se $|\alpha|<\left[\frac{f\left(x_{0}^{k}\right)-f\left(x_{0}^{k+1}\right)}{f\left(x_{m-1}^{k}\right)-f\left(x_{m}^{k}\right)}\right]^{\frac{1}{2}}$, então utilize as mesmas direções para a próxima busca univariada. Se a equação não for satisfeita, substituir a $m$-ésima direção pela direção padrão $\boldsymbol{s}_{p}^{k}$.

5) Comece a nova busca univariada com as direções obtidas no passo 4, e repita os passos 2, 3 e 4, até que a convergência seja atingida, ou seja:

$$
\left\|x_{k+1}-x_{k}\right\| \leq \varepsilon
$$

A Figura 4 ilustra o caminho seguido pelo método das direções conjugadas de Powell durante as iterações.

\footnotetext{
1 POWELL, M. J. . An efficient method for finding the minimum of a function of several variables
} without calculating derivatives. The Computer Journal, v. 7 (2), p. 155-162, 1964. 
Figura 4 - Evolução do processo de minimização pelo método de Powell.

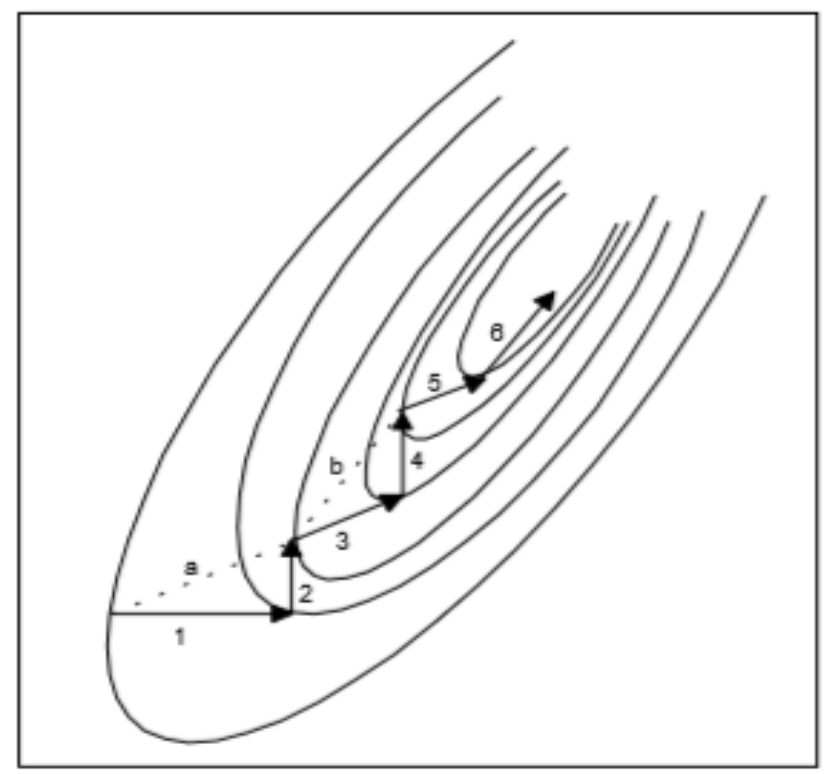

Fonte: HADDAD, 2001.

\subsection{Escolha do melhor modelo}

Depois de conhecer alguns métodos de predição, medidas de erro e processos de otimização, é usual buscar a avaliação de qual é o método mais adequado para a predição da demanda intermitente. A literatura, no entanto, traz uma avaliação de que este objetivo não é trivial e possui algumas variáveis importantes.

De acordo com Wallström e Segerstedt (2010), quando um erro de predição é mensurado, não é o método que é medido, mas a combinação de como este método responde a este erro. As relações entre diferentes erros variam de acordo com o método aplicado, o valor inicial e as constantes de suavizações utilizadas. Outras relações são as medidas de variância e de viés. Os autores descrevem também que, apesar do foco estar geralmente nos métodos de predição, estes podem estar relacionados a erros distorcidos, e por isto é relevante pesquisas que possam identificar quando um determinado erro pode ser usado ou não.

Estudos sobre a robustez dos métodos de predição, mostraram quão robustos são os métodos de predição para a demanda intermitente, considerando diferentes constantes de suavização, valores iniciais, dentre outros (WALLSTRÖM; SEGERSTEDT, 2010). Os autores concluíram que não existe, em geral, uma medida 
melhor, por duas razões principais. A primeira é a dimensão de um erro e a distorção de um erro em combinação com um método de predição. A segunda é que, sob certas circunstâncias, os erros favoreceram determinado tipo de viés. Por exemplo, o desvio médio absoluto (DMA) resultou em menor erro para um método de predição subestimado, utilizando-se a regressão logística quando os erros aumentam ou diminuem em relação às alterações das constantes de suavização. Por outro lado, Teunter e Duncan (2009) usaram a predição zero para descobrir como se comportam os diferentes erros.

As recomendações das pesquisas de Wallström e Segerstedt (2010), pesquisas correlatas de Teunter e Duncan (2009) e Kourentzes (2014) para a demanda intermitente estão descritas no Quadro 1.

Quadro 1- Quadro de recomendações de medidas de erro segundo Wallström e Segerstedt (2010) e outra pesquisas correlatas.

\begin{tabular}{|c|c|c|c|}
\hline Descrição & Medida & Recomendação & $\begin{array}{l}\text { Observações de Wallström e Segerstedt } \\
\text { (2010) e outras pesquisas correlatas }\end{array}$ \\
\hline DMA & Erro & $\begin{array}{l}\text { Inadequado, pois } \\
\text { distorce sob } \\
\text { circunstâncias de } \\
\text { maior intervalo } \\
\text { entre demandas } \\
\text { (demanda } \\
\text { intermitente). } \\
\text { Tendência de } \\
\text { predições zero } \\
\text { quando ocorre } \\
\text { otimização. }\end{array}$ & $\begin{array}{l}\text { Em Wallström \& Segerstedt (2010) o DMA } \\
\text { teve um comportamento distorcido para os } \\
\text { métodos. Para Croston e SBA, os itens } \\
\text { diminuíram DMA com o aumento da } \\
\text { constante de suavização, para as mesmas } \\
\text { variáveis significativas. } \\
\text { Para esta medida, os métodos de melhor } \\
\text { desempenho são os que tendem a } \\
\text { subestimar a demanda e, portanto, não é } \\
\text { considerada uma medida adequada para a } \\
\text { demanda intermitente. Desta forma, } \\
\text { concorda com a descoberta de Teunter e } \\
\text { Duncan (2009), que afirma que DMA } \\
\text { favorece a subestimação de métodos, e } \\
\text { que DQM também o faz, mas não no } \\
\text { mesmo grau. } \\
\text { Também concorda com a descrição de } \\
\text { Kourentzes (2014), de que esta medida } \\
\text { beneficia a predição ZERO de amostras } \\
\text { com demandas intermitentes. }\end{array}$ \\
\hline DQM & Erro & Estável suficiente & $\begin{array}{l}\text { DQM não mostrou qualquer } \\
\text { comportamento distorcido, como a } \\
\text { redução do erro com um aumento da }\end{array}$ \\
\hline
\end{tabular}




\begin{tabular}{|c|l|c|l|}
\hline & & $\begin{array}{l}\text { constante de suavização. DQM foi, } \\
\text { portanto, considerada uma medida } \\
\text { confiável (WALLSTRÖM; SEGERSTEDT, } \\
\text { 2010). }\end{array}$ \\
\hline PEE & Viés & $\begin{array}{c}\text { Adequado em } \\
\text { combinação com } \\
\text { outros erros de } \\
\text { polarização em vez } \\
\text { do uso de uma } \\
\text { única medida. }\end{array}$ & $\begin{array}{l}\text { PEE está correlacionado com DQM } \\
\text { mesmo sem os valores extremos. O PEE } \\
\text { foi a medida que forneceu o máximo de } \\
\text { informações sobre os erros de predição } \\
\text { adicionais, uma vez que acrescenta outra } \\
\text { dimensão, tempo, à medida do erro } \\
\text { (WALLSTRÖM; SEGERSTEDT, 2010). }\end{array}$ \\
\hline
\end{tabular}

Fonte: WALLSTRÖM; SEGERSTEDT (2010); TEUNTE; DUNCAN (2009); KOURENTZES (2014).

Quanto aos métodos testados por Wallström e Segerstedt (2010), nenhum deles se destacou como mais adequado para DQM e DMA. Mas, em pesquisas anteriores, os métodos SBA e Croston, provaram ser os mais estáveis e menos sensíveis à escolha da constante de suavização. O método de Croston também foi apropriado para demanda suave e para medida de erro por meio do DQM (SYNTETOS; BOYLAN, 2005).

Existem, portanto, diferenças entre os métodos de predição, entre diferentes valores para os mesmos métodos de predição, e entre diferentes constantes de suavização para o mesmo método e valor inicial. Em geral, as relações são mais estáveis dentro de um mesmo método do que entre os métodos. As medidas convencionais usadas são medidas de variação ou desvio e, portanto, não são semelhantes ao nível de serviço, impacto no inventário ou de custo. Desta forma, para avaliar um erro, existem pelo menos quatro dimensões de interesse: variação, tendência, serviço e impacto no estoque e custo (WALLSTRÖM; SEGERSTEDT, 2010).

Lee e Everett (1986) chegaram à conclusão de que no processo de manufatura, uma superestimação é preferida devido a menos configurações e, portanto, menor o custo. Porém, quando a quantidade em estoque é o maior foco, uma subestimação pode ser melhor se o estoque de segurança for baseado no desempenho da variação.

A escolha do método de predição não deve se basear apenas no erro de predição, mas também sobre as consequências para a organização do método escolhido. A escolha deve refletir as estratégias da organização, embora reconheça 
que é mais fácil escrever esse objetivo do que implementá-lo na prática. Em logística, existe uma tendência de sempre minimizar o custo em vez de aumentar o lucro. Às vezes, a minimização cria uma sub otimização, seja na própria organização ou na cadeia de suprimentos (WALLSTRÖM; SEGERSTEDT, 2010).

Segue na Figura 5, um resumo da evolução de algumas pesquisas referenciadas neste trabalho. 
Figura 5 - Resumo da revisão da literatura.

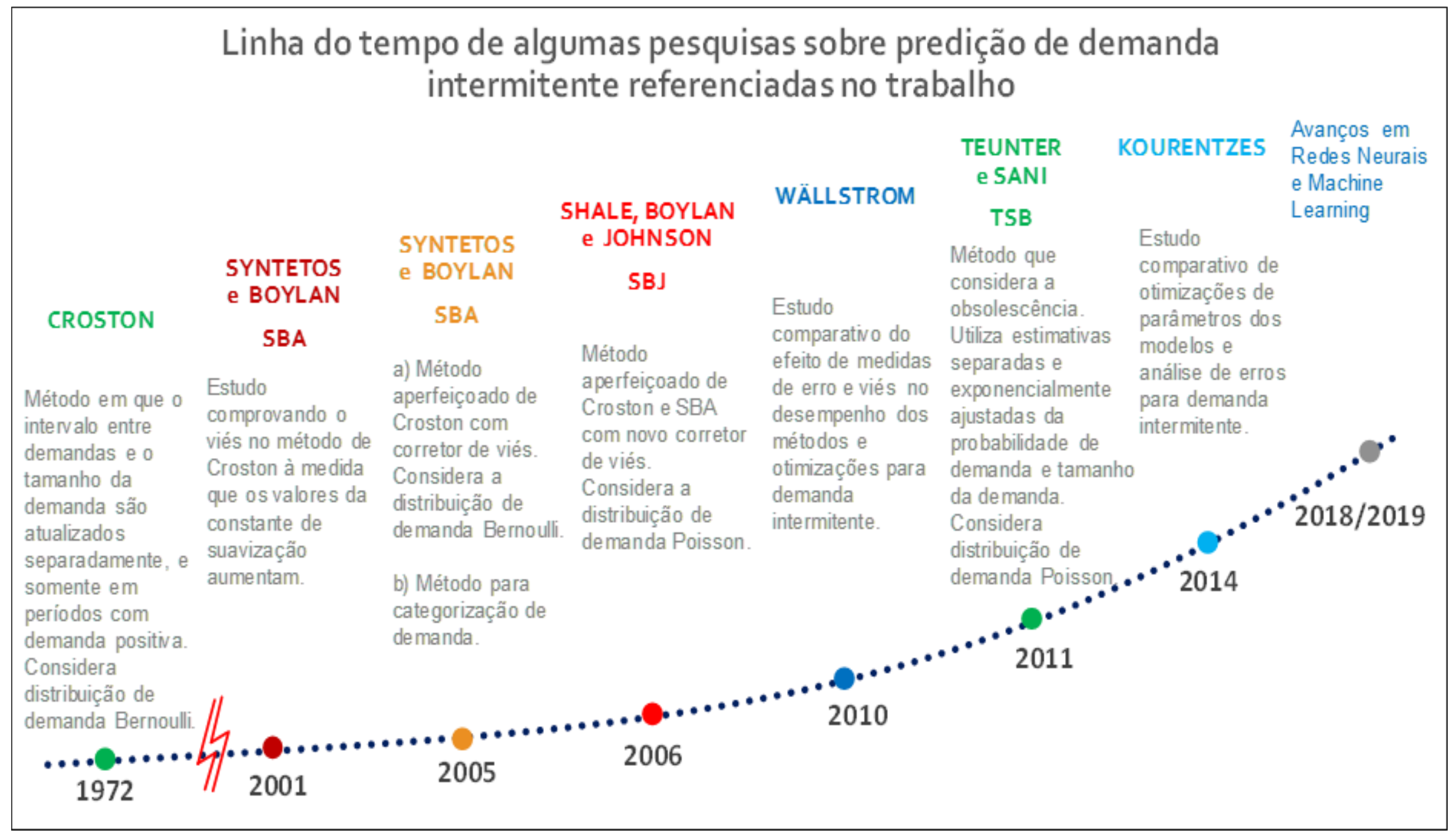

Fonte: O autor, 2020. 


\section{METODOLOGIA}

Este trabalho foi realizado em três etapas:

i. Abordagem descritiva, com o objetivo de descrever os fatos, analisá-lo, classificá-los e interpretá-los, sem interferência do pesquisador.

ii. Abordagem exploratória, visando proporcionar maior familiaridade com o problema em pauta.

iii. Abordagem prática, visando solucionar um problema a partir da observação de dados reais.

A abordagem da pesquisa pode ser categorizada como quantitativa, pois requer o uso de recursos matemáticos e técnicas estatísticas para análise do problema proposto.

Esta pesquisa trata da predição de demanda de itens relacionados ao setor de manutenção de equipamentos de mineração, embora seja aplicável a outros setores cuja demanda por peças, produtos e itens tenham comportamento de consumo majoritariamente intermitente.

A análise trata da classificação de itens com diversos comportamentos de consumo, porém concentrando as análises nos itens de natureza intermitente, utilizando os métodos apresentados nos objetivos específicos.

\subsection{Predição de demandas de peças de reposição}

A predição de demandas de peças de reposição do setor de mineração foi realizada por meio de simulação, utilizando as abordagens de Croston (1972); Syntetos e Boylan (2005); Shale, Boylan e Johnston (2006); e Teunter, Syntetos e Babai (2011). As análises e simulações seguiram os procedimentos descritos no organograma da Figura 6. 
Figura 6 - Organograma do processo de simulação e análise de predições.

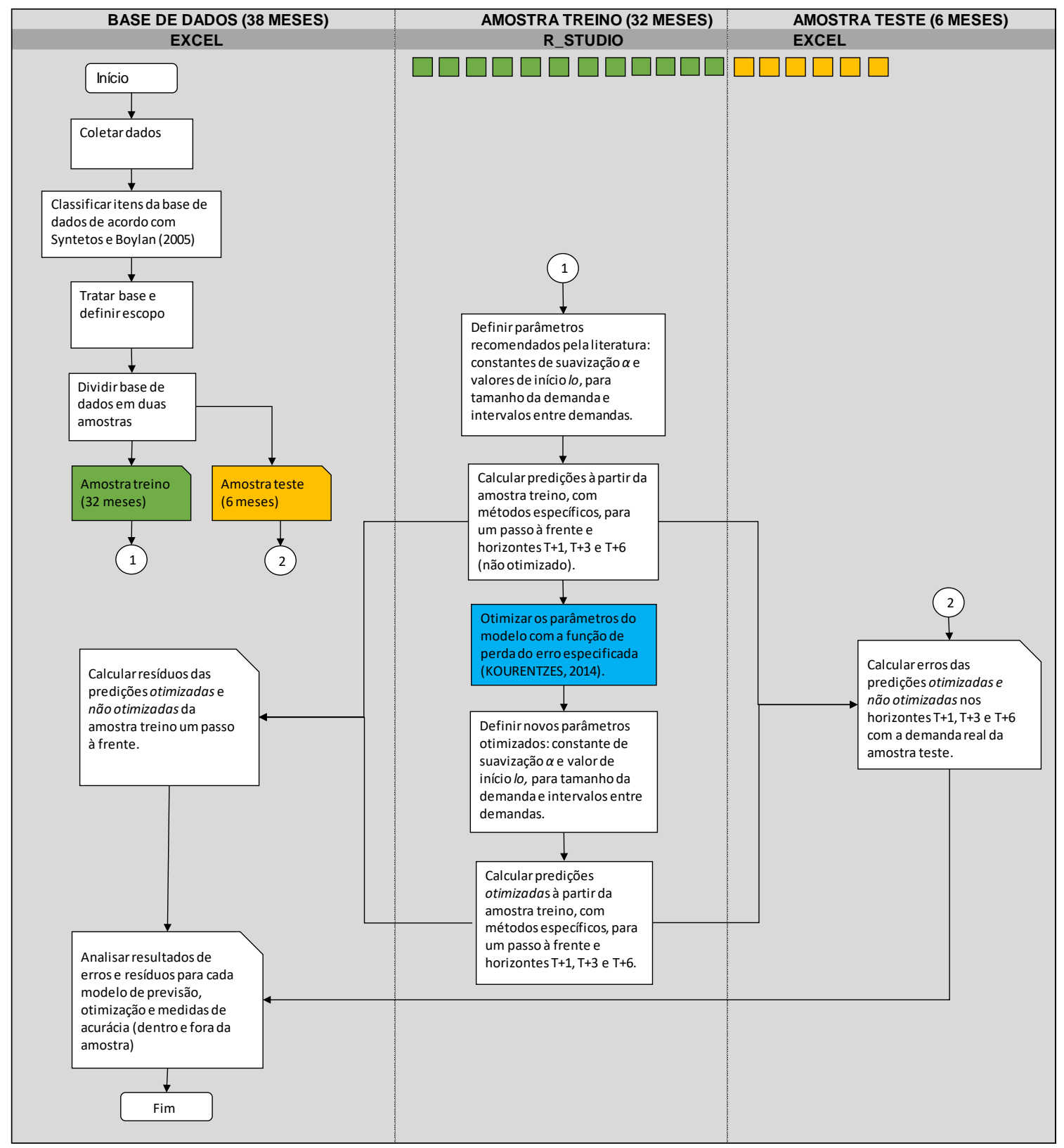

Fonte: O autor, 2020.

\subsubsection{Base de dados}

A base de dados para realização do trabalho foi fornecida a partir do sistema informatizado de uma empresa do seguimento de mineração. A base é constituída por um conjunto de dados mensais referente ao consumo de itens de Manutenção, Reparo e Operação (MRO). Por questões de confidencialidade, não foram fornecidas informações adicionais sobre os itens, sendo de interesse da pesquisa 
apenas a sua série histórica. Foi realizada uma coleta quantitativa a partir do levantamento de informações do sistema ERP, para uma amostra correspondente a 2.403 itens, seguindo os critérios descritos nesta Seção. A base coletada permitiu que os métodos de predição fossem aplicados, treinados e testados, sem comprometer os resultados.

A amostra utilizada corresponde a um período de 38 meses, de janeiro de 2016 a fevereiro de 2019, e a base de dados foi dividida em duas partes. A primeira constitui um conjunto denominado de amostra de treino, contendo uma série temporal de 32 meses. A segunda parte, um conjunto denominado de amostra de teste, contendo um conjunto de séries temporais de 6 meses.

O período da amostra foi dividido com uma aproximação de $80 \%$ para treino e 20\% para teste, de acordo com recomendação de Hyndman e Athanasaupolos (2018), em que é recorrente a utilização do valor de $20 \%$ da amostra total de dados para teste. Este percentual não é fixo e pode ser alterado em função do tamanho do conjunto de dados e da extensão da predição que se pretende realizar.

O período de demanda observada de 32 meses foi utilizado para predições de um passo à frente e suas otimizações, e o período posterior de 6 meses foi usado para aferição entre a predição e o consumo observado na amostra teste. O período de 6 meses para teste foi dividido em 3 horizontes: um mês, três meses e seis meses.

Outra etapa de relevância para o trabalho foi o processamento dos dados em um formato para análise. Em geral, as informações obtidas em formato bruto precisam ser entendidas e tratadas adequadamente antes de sua utilização. Para este procedimento, alguns cuidados foram necessários na seleção dos itens a serem utilizados.

O primeiro cuidado foi escolher a unidade do item de consumo, em que foi utilizada apenas a unidade "peça", excluindo-se os itens que não fossem peças de reposição como óleos (L), lubrificantes $(\mathrm{kg})$, serviços (ud.), dentre outros. Também foi utilizado o critério de não selecionar itens sem consumo nos últimos 12 meses e/ou nos primeiros 12 meses da amostra de treino. Este procedimento visou excluir itens obsoletos e itens novos com menor histórico para aplicação dos métodos.

A agregação temporal adotada foi a mensal, em que se considerou pouca perda nas séries temporais, pois a natureza dos itens é, em sua maioria, de alta intermitência, sem observar fatores como sazonalidade e tendência. 
O critério utilizado para os itens "elegíveis" foi a ocorrência de consumo maior ou igual a 2 eventos no período de 32 meses, excluindo-se os demais itens. Efetuouse uma segregação apenas de itens industriais, para que fossem avaliados quanto ao comportamento da demanda e aplicabilidade dos métodos de predição. Isto foi feito separando-se as plantas industriais dos outros setores da empresa, com atenção especial para evitar plantas e equipamentos paralisados temporariamente ou desativados. Por meio destes filtros, foram selecionados os 2.403 Unidades de Manutenção de Estoque - UME (Stock Keeping Unit - SKU) da amostra bruta. É importante destacar que, apesar de se utilizar estes filtros que reduziram a base de dados bruta, os benefícios da base tratada são mais importantes para os objetivos do trabalho. Foram elaboradas, posteriormente, tabelas de consumo mensal de itens estratificadas, como descrito a seguir:

- Período de 32 meses-Base de dados observados de consumo, utilizada para treino dos modelos com simulações de predição para um passo à frente e para horizontes $\mathrm{T}+1, \mathrm{~T}+3$ e $\mathrm{T}+6$ meses.

- Período de 6 meses-Base de dados observados de consumo, utilizada para teste e aferição dos resultados das predições nos horizontes $\mathrm{T}+1, \mathrm{~T}+3$ e $\mathrm{T}+$ 6 meses, usando medidas de erro.

\subsubsection{Tratamento de descontinuidade da base de dados}

A descontinuidade é observada quando faltam dados em determinados períodos. No caso deste trabalho, foi observada a falta de dados em um mês no período analisado. Para contornar este problema, o dado omisso foi obtido por meio de uma interpolação linear. Este procedimento foi realizado com critério conservador, para não "perturbar" os resultados e induzir o comportamento da amostra.

\subsubsection{Classificação da demanda de itens}

A classificação da demanda de itens foi realizada com base nas técnicas de intervalo de tempo entre demandas e tamanho da demanda diferente de zero, categorizando-a conforme os valores do quadrado do coeficiente de variação $\left(c_{v}^{2}\right)$ e 
no intervalo médio de demanda ( $p$ ) (SYNTETOS; BOYLAN, 2005), descrito na Seção 1.3.1., e ilustrado na Figura 7.

Figura 7- Exemplo ilustrativo de categorização de demanda de itens da amostra.

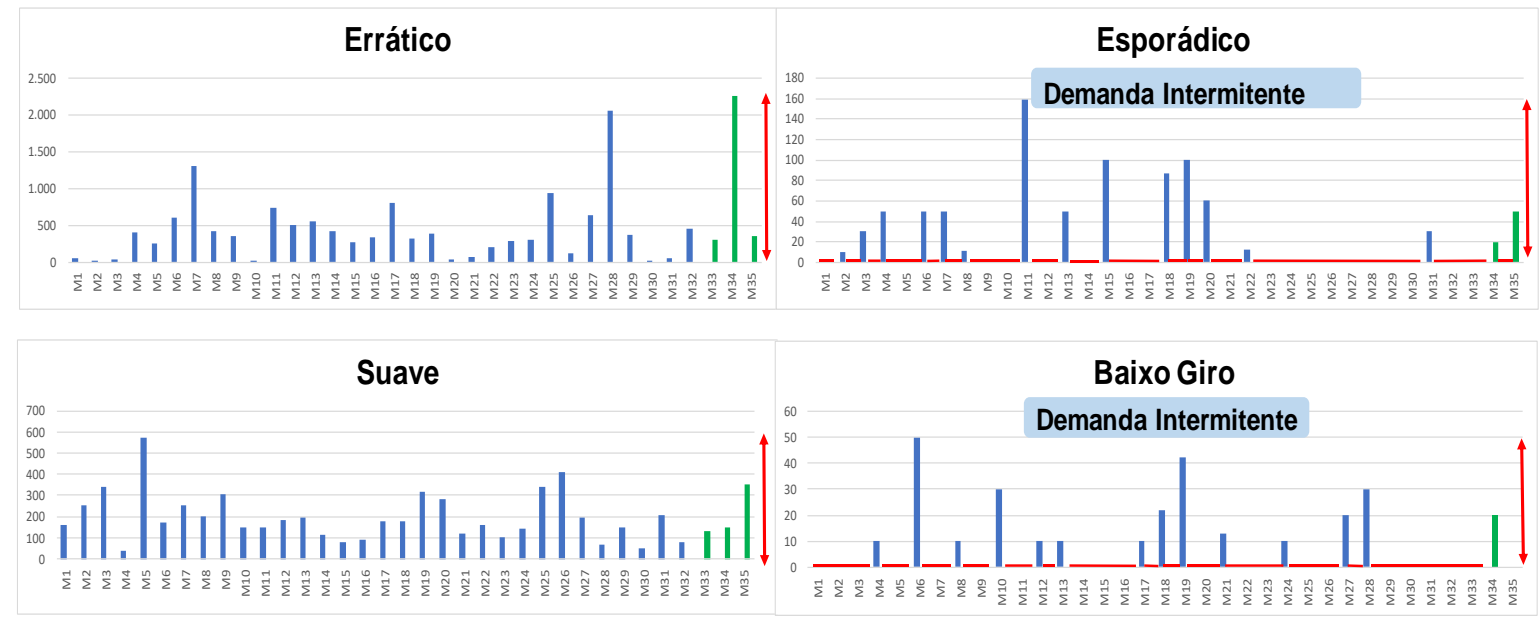

Fonte: O autor, 2020.

A média dos intervalos entre demandas $(p)$ foi obtida utilizando a Equação (22) e o quadrado do coeficiente de variação dos tamanhos de demanda $\left(c_{v}^{2}\right)$ utilizando a Equação (23).

$$
\begin{gathered}
p=\frac{1}{N} \sum_{i=1}^{N} q_{i} \\
c_{v}^{2}=\left(\frac{\sigma}{\mu}\right)^{2}
\end{gathered}
$$

Em que:

$p=$ Intervalo médio entre demandas;

$q_{i}=$ Intervalo desde a última demanda;

$N=$ Número de intervalos entre demandas;

$c_{v}=$ Coeficiente de variação da população;

$\sigma=$ Desvio padrão da população;

$\mu=$ Média da população.

Os pontos de divisão nos eixos (valores de corte) para a categorização de demanda foram $p=1,32$ e $c_{v}^{2}=0,49$, conforme Syntetos e Boylan (2005). 


\subsection{Modelos de predição}

A predição de demanda de peças foi realizada por meio de simulação, utilizando os métodos convencionais de predição de demanda Média Móvel Simples (MMS) e Suavização Exponencial Simples (SES). Foram utilizados também os estimadores de Croston (CROSTON, 1972), o método SBA (SYNTETOS; BOYLAN, 2005), o método SBJ (SHALE; BOYLAN; JOHNSTON, 2006) e o método TSB (TEUNTER; SYNTETOS; BABAI, 2011).

Para comparação e referência dos resultados de predições, ou por ser parte integrante de algumas medidas de erro (por exemplo, o erro médio absoluto escalonado - EMAE), o método "ingênuo" (NAÏVE) e o método de predição "zero" também foram considerados.

Segue detalhamento dos métodos utilizados:

\subsubsection{Método de Predição Zero}

É um método em que se estima, para cada horizonte, uma predição de valor zero:

$$
\hat{y}_{t}=0
$$

Em que $\hat{y}_{t}$ é a predição de demanda para o período $t$.

\subsubsection{Método Ingênuo (Naïve)}

O método ingênuo é utilizado como método de referência (benchmarking) e considera que as predições futuras são iguais ao último valor observado:

$$
\hat{y}_{t}=y_{t-1}
$$

Em que:

$\hat{y}_{t}=$ Predição de demanda para o período $t$;

$y_{t-1}=$ Demanda observada no período anterior a $t$. 
O método da Média Móvel Simples (MMS) consiste em calcular a média aritmética de uma série de $n$ observações:

$$
\hat{y}_{t}=\frac{1}{n} \sum_{i=1}^{n} y_{t-i}
$$

Em que:

$\hat{y}_{t} \quad=$ Predição de demanda para o períodot;

$y_{t-i}=$ Demanda observada no período $t-i$;

$n \quad=\quad$ Número de períodos $t$.

1.8.4 Método da Suavização Exponencial Simples (SES)

A estimação da demanda pelo método SES é feita utilizando as expressões (MAKRIDAKIS; WHEELRIGHT; HYNDMAN, 1998):

$$
\begin{gathered}
\hat{y}_{t}=\hat{y}_{t-1}+\alpha\left(y_{t-1}-\hat{y}_{t-1}\right) \\
\hat{y}_{t}=\alpha \cdot y_{t-1}+(1-\alpha) \hat{y}_{t-1}
\end{gathered}
$$

Em que:

$\hat{y}_{t} \quad=$ Predição da demanda para o períodot;

$\hat{y}_{t-1}=$ Predição da demanda para o período anterior a $t$;

$y_{t-1}=$ Demanda observada para o período anterior at;

$\alpha=$ Declividade da curva de $\hat{y}_{t}$ (constante entre 0 e 1 ).

\subsubsection{Método de Croston (CRO)}

Neste modelo, o autor assume que a demanda ocorre como um processo de Bernoulli e o intervalo entre demandas segue uma distribuição geométrica. Desta forma, a estimativa de demanda pode ser dada por:

$$
y_{t}=x_{t} \cdot z_{t}
$$




$$
x_{t}=\left\{\begin{array}{rrr}
1, & \text { com prob }\left(\frac{1}{p}\right) \text { quando a demanda ocorre } \\
0, & \text { ou } & \text { com } \operatorname{prob}\left(1-\frac{1}{p}\right)
\end{array}\right\}
$$

O parâmetro $\mathrm{z}_{\mathrm{t}}$ segue uma distribuição normal com média $\mu$ e variância $\sigma^{2}$ :

$$
\mathrm{z}_{\mathrm{t}} \sim \mathrm{N}\left(\mu, \sigma^{2}\right)
$$

De acordo com o método de Croston, todos os estimadores são atualizados apenas quando ocorrer uma demanda.

Se $y_{t}=0$, tem-se:

$$
\hat{p}_{t}=\hat{p}_{t-1} ; \quad \hat{z}_{t}=\hat{z}_{t-1} ; \quad q=q+1
$$

Se $y_{t} \neq 0$, tem-se:

$$
\hat{p}_{t}=\hat{p}_{t-1} \alpha\left(\mathrm{q}-\hat{p}_{t-1}\right) ; \quad \hat{z}_{t}=\hat{z}_{t-1}+\alpha\left(\mathrm{y}_{\mathrm{t}}-\hat{z}_{t-1}\right) ; \quad \mathrm{q}=1
$$

Em que:

$q$ = Intervalo desde a última demanda;

$z_{t}=$ Tamanho da demanda;

$p=$ Intervalo entre demandas;

$p_{t}=$ Representa a ocorrência de demanda;

$\hat{p}_{t}=$ Intervalo entre demanda, suavizado exponencialmente, atualizado apenas se a demanda ocorrer no período $t$;

$\hat{z}_{t}=$ Tamanho da demanda exponencialmente suavizado, atualizado somente se a demanda ocorrer no período $t$.

De acordo com o procedimento de estimativa em que $0 \leq \alpha \leq 1$ é uma constante de suavização, $\hat{y}_{t}$ é a demanda predita no período $t$, dada pela Equação (34): 


$$
\hat{y}_{t}=\frac{\hat{z}_{t}}{\hat{p}_{t}}
$$

\subsubsection{Método de Aproximação de Syntetos e Boylan (SBA)}

Com o intuito de aperfeiçoar o método de Croston (1972), foi apresentado por Syntetos e Boylan (2005) o estimador definido como Aproximação de Boylan e Syntetos (SBA), descrito na Equação (35).

$$
\hat{y}_{t}=\left(1-\frac{\alpha}{2}\right)\left(\frac{\hat{z}_{t}}{\hat{p}_{t}}\right)
$$

\subsubsection{Método de Shale, Boylan e Johnston (SBJ)}

Shale, Boylan e Johnston (2006) mostraram que, se a quantidade de pedidos de um determinado item seguir um processo de Poisson, um estimador mais apropriado é dado pela Equação (36), que define o método SBJ.

$$
\hat{y}_{t}=\left(1-\frac{\alpha}{2-\alpha}\right)\left(\frac{\hat{z}_{t}}{\hat{p}_{t}}\right)
$$

\subsubsection{Método de Teunter, Syntetos e Babai (TSB)}

O método TSB utiliza estimativas separadas e exponencialmente ajustadas do tamanho da demanda e da probabilidade de demanda. A estimativa da probabilidade de ocorrência é atualizada a cada período. A estimativa do tamanho da demanda é atualizada apenas no final dos períodos com demanda positiva, de acordo com as equações (37), (38) e (39).

Se $y_{t}=0$ :

$$
\hat{p}_{t}=\hat{p}_{t-1}+\beta\left(0-\hat{p}_{t-1}\right) ; \quad \hat{z}_{t}=\hat{z}_{t-1}
$$


Se $y_{t}>0$ :

$$
\begin{gathered}
\hat{p}_{t}=\hat{p}_{t-1}+\beta\left(1-\hat{p}_{t-1}\right) ; \quad \hat{z}_{t}=\hat{z}_{t-1}+\alpha\left(\mathrm{z}_{\mathrm{t}}-\hat{z}_{t-1}\right) \\
\hat{y}_{t}=\hat{p}_{t} \cdot \hat{z}_{t}
\end{gathered}
$$

Para as otimizações dos modelos, foi utilizado o programa específico tsintermittent, que faz parte da plataforma de programação $R$-Studio, e o Microsoft Excel 2016. Um exemplo de aplicação destas sub-rotinas é ilustrado na Figura 8.

Figura 8 - Exemplo de aplicação de modelos de predição usando R-tsintermittent.
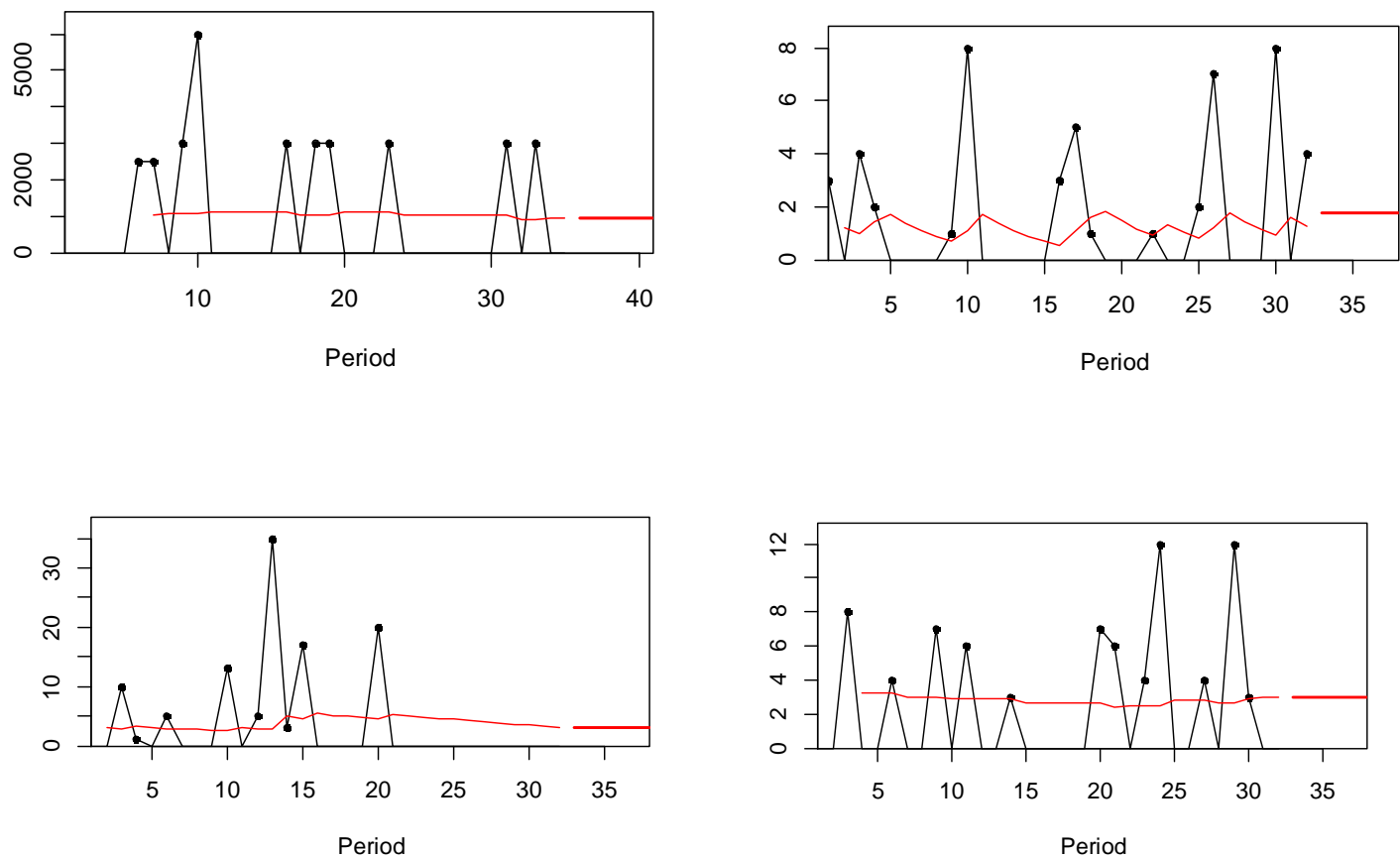

Fonte: O autor, 2020.

\subsection{Avaliação de desempenho}

Para a avaliação de desempenho foram utilizadas as versões escalonadas das medidas de acurácia descritas na Seção 1.4., obtidas pela divisão do valor da medida de erro pela média da amostra. O erro escalonado é um erro relativo, cujo objetivo é a remoção da escala, para que os erros de diferentes séries temporais possam ser comparados. 
As medidas de erros utilizadas para comparar o desempenho de cada método de predição de demanda e para minimização das funções de perda, foram:

O Desvio Médio Absoluto Escalonado (DMAE), que consiste no módulo da diferença entre o valor real e o estimado, dividindo-se pela média da amostra:

$$
\bar{D}_{a b s E}=\frac{1}{n}\left(\frac{\sum_{t=1}^{n}\left|y_{t}-\hat{y}_{t}\right|}{\frac{1}{n} \sum_{i=1}^{n} y_{i}}\right)
$$

Em que:

$$
\begin{array}{ll}
\bar{D}_{a b s E} & =\text { Desvio médio absoluto escalonado (DMAE); } \\
y_{t} & =\text { Valor observado (ou real) para o período } t ; \\
\hat{y}_{t} & =\text { Valor predito (ou estimado) para o período } t ; \\
n & =\text { Número de observações; } \\
y_{i} & =\text { Valor observado (ou real). }
\end{array}
$$

O Desvio Quadrático Médio Escalonado (DQME), que consiste no quadrado da diferença entre o valor real e o estimado, dividindo-se pela média da amostra:

$$
\bar{D}_{\text {quadE }}=\frac{1}{n}\left(\frac{\sum_{i=1}^{n}\left(y_{t}-\hat{y}_{t}\right)}{\frac{1}{n} \sum_{i=1}^{n} y_{i}}\right)^{2}
$$

Em que:

$$
\begin{array}{ll}
\bar{D}_{\text {quadE }} & =\text { Desvio quadrático médio escalonado (DQME); } \\
y_{t} & =\text { Valor observado (ou real) para o período } t ; \\
\hat{y}_{t} & =\text { Valor predito (ou estimado) para o período } t ; \\
n & =\text { Número de observações; } \\
y_{i} & =\text { Valor observado (ou real). }
\end{array}
$$

O Erro Médio Absoluto Escalonado (EMAE), obtido pela divisão do erro da predição pela média do erro do método ingênuo (NAÏVE), usando as Equações (3) e (4) da Seção 1.4. 
Medidas de erro relacionadas ao inventário também foram utilizadas: Período em Estoque Escalonado (PEEE), Período em Estoque Escalonado Absoluto Modificado (PEEEAm), Taxa Absoluta Média Escalonada (TAME) e Taxa do Quadrado da Média Escalonada (TQME). Estas são medidas de somatório acumulativo, que visam impedir a preferência pelas predições de demanda zero em uma série temporal muito intermitente.

Uma versão escalonada foi obtida dividindo-se o PEE pela média da amostra:

$$
P_{E E E}=\frac{P_{E E}}{\frac{1}{n} \cdot \sum_{i=1}^{n} y_{i}}
$$

Em que:

$$
\begin{array}{ll}
P_{E E E} & =\text { Períodos em estoque escalonado (PEEE); } \\
P_{E E} & =\text { Períodos em estoque (PEE); } \\
y_{i} & =\text { Valor observado (ou real); } \\
n & =\text { Número de observações. }
\end{array}
$$

Esta medida foi considerada como uma métrica de viés e não como medida de erro, sendo aplicável em combinação a outras medidas (KOURENTZES, 2014; WALLSTRÖM; SEGERSTEDT, 2010).

O Período em Estoque Escalonado Absoluto Modificado (PEEEAm) foi obtido pela divisão do módulo do período em estoque pela demanda média da amostra de cada item, conforme Equação (6) da Seção 1.4.

Outras metodologias para otimização são derivadas da propriedade da taxa acumulativa do erro $\left(r_{i}\right)$, calculada pela Equação (7) da Seção 1.4. Duas versões escalonadas foram determinadas dividindo-se as taxas médias absolutas e quadráticas pela média da amostra, conforme Equações (43) e (44):

$$
\bar{T}_{a b s E}=\left(\frac{\sum_{i=1}^{n}\left|r_{i}\right|}{\frac{1}{n} \sum_{i=1}^{n} y_{i}}\right)
$$




$$
\bar{T}_{\text {quadE }}=\left(\frac{\sum_{i=1}^{n} r_{i}^{2}}{\frac{1}{n} \sum_{i=1}^{n} y_{i}}\right)
$$

Em que:

$$
\begin{array}{ll}
\bar{T}_{a b s E} & =\text { Taxa média absoluta escalonada (TAME); } \\
r_{i} & =\text { Taxa acumulativa do erro; } \\
n & =\text { Número de observações; } \\
y_{i} & =\text { Valor observado (ou real); } \\
\bar{T}_{\text {quadE }} & =\text { Taxa do quadrado da média escalonada (TQME). }
\end{array}
$$

\subsection{Otimização dos modelos}

Para uma solução algébrica de otimização, através da obtenção de $\alpha$ e $l_{0}$ ótimos, chegou-se ao desdobramento da Equação (45) para qualquer série temporal deste trabalho com função de perda SES:

$$
\mathrm{DQM}=\frac{1}{32} \sum_{t=1}^{32}\left\{y_{\mathrm{t}}-\sum_{j=1}^{32} \alpha(1-\alpha)^{32-\mathrm{j}} \cdot y_{\mathrm{j}}+(1-\alpha)^{32} \cdot l_{0}\right\}^{2}
$$

O problema descreve a otimização ou procura por um valor mínimo de uma função multivariável, que implica no cálculo do erro dentro do intervalo de todas as combinações possíveis de constante de suavização e valores iniciais de predição. Como não é prático resolver, algebricamente, este problema de minimização, principalmente para outros modelos além de SES, que possuem mais parâmetros variáveis, optou-se por um algoritmo numérico específico, que conseguisse reduzir os cálculos e esforço computacional.

Para otimizar os parâmetros $\alpha$ (suavização exponencial) e $l_{0}$ (valor inicial em $t=0$ ) optou-se pela sub-rotina específica tsintermittent, que faz parte da plataforma de programação $R$-Studio, para encontrar os menores valores de erro das funções de perda especificadas no programa, que são DMA, DQM, TAM e TQM. A vantagem deste programa está na possibilidade de realizar as predições simultâneas para uma quantidade maior de itens, aspecto importante em comparação com a sub-rotina solver disponibilizado no Excel, por exemplo. 
O R-Studio também disponibiliza bibliotecas específicas que atendem à proposta de trabalho. Apesar de cada biblioteca poder utilizar metodologias diferentes de otimização, optou-se pela tsintermittent, que está associada a artigos de pesquisas recentes. A versão utilizada da biblioteca tsintermittent foi desenvolvida por Nikola os Kourentzes e Fotios Petropoulos em 2016 e possui funções para análise de predição de demanda intermitente. Neste trabalho, foram utilizadas as funções dos modelos SES, CROSTON, SBA, SBJ e TSB.

Também foi implementada a otimização dos parâmetros da constante de suavização e valores iniciais de demanda e intervalo, conforme Kourentzes (2014). Na metodologia utilizada, os valores iniciais e constante de suavização dos modelos foram otimizados por meio da minimização de cada uma das funções de perda DMA, DQM, TAM e TQM.

\subsubsection{Análise de erros dos modelos}

Para análise dos erros dos modelos foram utilizadas as seguintes medidas: Desvio Médio Absoluto Escalonado (DMAE), Desvio Quadrático Médio Escalonado (DQME), Erro Médio Absoluto Escalonado (EMAE), Período em Estoque Escalonado Absoluto Modificado (PEEEAm), Taxa do Quadrado da Média Escalonada (TQME), e Taxa Média Absoluta Escalonada (TAME). O Período em Estoque Escalonado (PEEE) foi utilizado para combinação de resultados, por se tratar de uma medida de viés, e não medida de erro(KOURENTZES, 2014; WALLSTRÖM; SEGERSTEDT, 2010).

Por causa da sua praticidade na visualização de resultados, e por possuir funções que permitem simular modelos mais simples de predição, o programa Excel 2016 foi escolhido para estimação dos erros. Os modelos com base em Média Móvel, Ingênuo (Naïve) e predição zero, não estavam presentes plataforma $R$-Studio utilizada. Então, a sub-rotina Solver do Excel 2016 também contribuiu para aferir os resultados das simulações, alguns a totalidade e outros por amostragem.

$A$ validação dos modelos de predição de erros um passo à frente, foi realizada para todos os períodos da amostra de treino. A validação dos modelos de predição de erros de teste foi realizada para três horizontes temporais: mensal, trimestral e semestral. 


\subsubsection{Análise dos resultados}

A análise dos resultados foi realizada comparando-se os resultados obtidos com a otimização com aqueles obtidos com base no desempenho dos métodos de predições, para cada medida de erro. A forma de apresentação escolhida foi uma classificação de posições, tomando como referência os erros (amostra de teste) e os resíduos (amostra de treino) encontrados, usando-se o critério de quanto menor a posição na classificação, melhor o desempenho da otimização ou do modelo.

Para itens com demanda de alto giro, em menor quantidade na amostra (380 itens), foi realizada uma comparação para aferir e identificar possíveis padrões de comportamento de erros, similares ou inversos à demanda intermitente.

Por fim, foram analisados os efeitos da otimização nos parâmetros $\alpha$ de cada modelo e a relação entre eles. Para isto, a partir das simulações, foram comparados os resultados das variações das médias das constantes de suavização $\alpha$, com o auxílio do programa Minitab. 


\section{RESULTADOS E DISCUSSÃO}

Neste capítulo são apresentados e analisados os resultados deste trabalho, obtidos de acordo com a metodologia apresentada.

\subsection{Resultados das predições de demanda}

De acordo com a base de dados, primeiramente é apresentada a classificação das séries da amostra utilizada (SYNTETOS; BOYLAN, 2005), conforme ilustrada na Figura 9.

Figura 9 - Classificação da demanda da amostra.

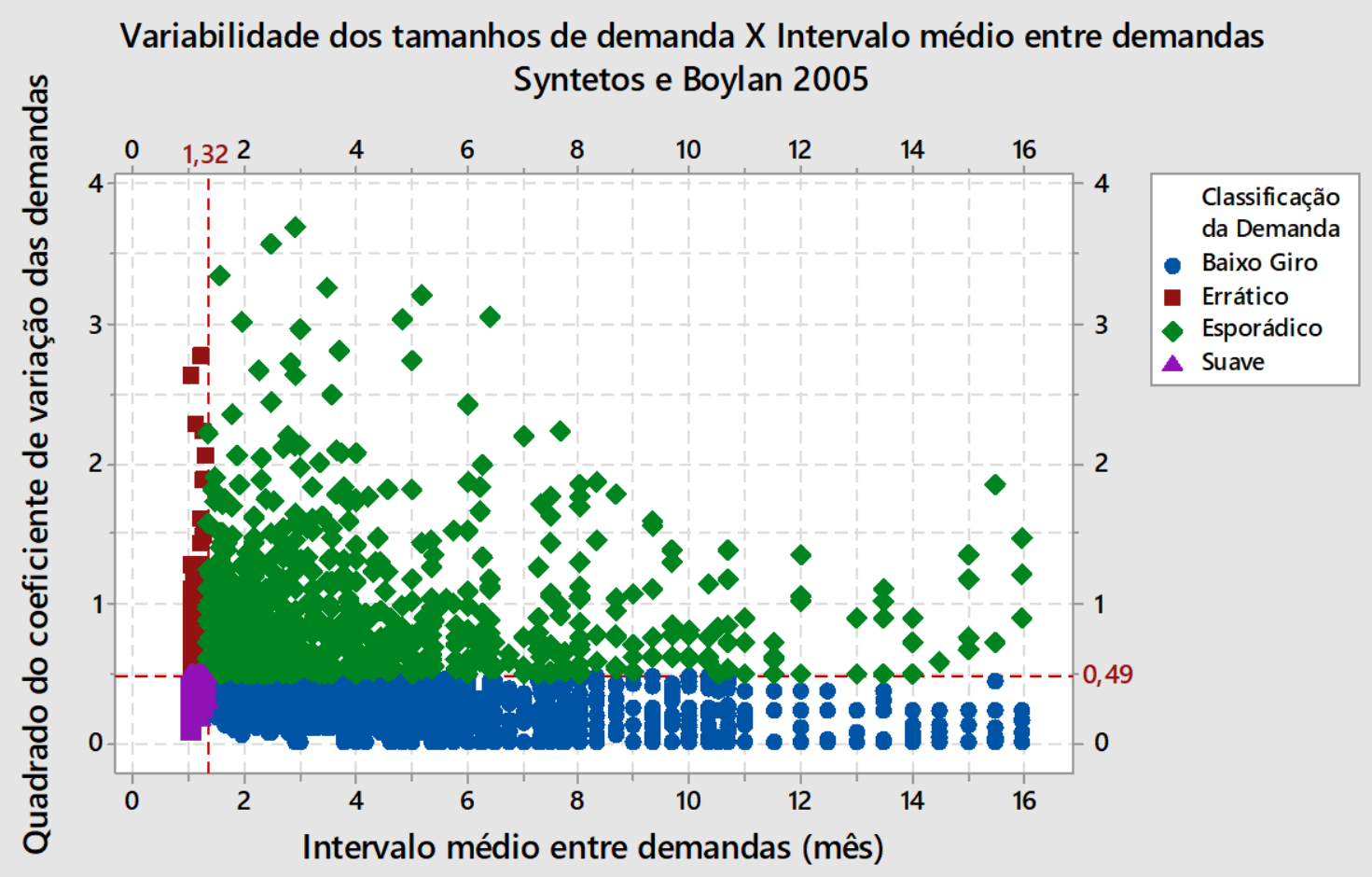

Fonte: O autor, 2020.

Observou-se que $84 \%$ dos itens, que correspondem a 2.023 Unidades de Manutenção de Estoque - UME (Stock Keeping Unit - SKU), possuíam demandas classificadas como de baixo giro ou esporádicas, o que configura um cenário majoritariamente formado por séries intermitentes. Apesar de não estar sendo avaliados valores de peças de reposição e sim a quantidade, este resultado sinaliza 
uma possível conformidade com o fato de que esses itens podem representar até $60 \%$ do valor total do estoque (JOHNSTON; BOYLAN; SHALE, 2003), o que reforça a necessidade de pesquisas ligadas a este tipo de demanda.

Para os resultados das predições, optou-se pela avaliação de desempenho utilizando as medidas de erro: Desvio Médio Absoluto Escalonado (DMAE), Desvio Quadrático Médio Escalonado (DQME), Erro Médio Absoluto Escalonado (EMAE), Período em Estoque Escalonado Absoluto modificado (PEEEAm), Taxa do Quadrado da Média Escalonada (TQME) e a Taxa Absoluta Média Escalonada (TAME).

Foram analisados os resultados das otimizações dos parâmetros de suavização pela minimização das medidas de erro DMA, DQM, TAM e TQM usando a sub-rotina tsinttermitent disponível no $R$-Studio. Os resultados dos modelos também foram comparados com os obtidos utilizando parâmetros convencionais indicados na literatura. Para as suavizações exponenciais utilizou-se a constante de suavização $\alpha=0,1$; para TSB utilizou-se $\beta=0,2$; além de valores iniciais do intervalo entre demandas com método ingênuo (naïve) e média.

Para melhor visualização dos resultados, foram calculados os resíduos da amostra de "treino" (32 meses) e dos erros das amostras de "teste" para 1 mês, 3 meses e 6 meses. Os métodos envolvidos na análise foram: MMS (de 3 meses), SES, CROSTON, SBA, SBJ, TSB e para referência ZERO e NAÏVE.

As análises trazem a posição na classificação de erros das otimizações e modelos de predição, considerando dois grupos de medidas associadas por proximidade no resultado. Quanto menor a posição na classificação, melhor o desempenho da otimização, que estão destacados em negrito. A seguir apresentamse os resultados das simulações para séries de demanda intermitente em relação ao primeiro grupo de medidas.

1.11.1 Resultado do Primeiro Grupo de medidas de erro para demanda intermitente

Para esta análise foi considerada a classificação da amostra de treino, com um passo à frente, e da amostra de teste com três horizontes à frente: um, três e seis meses. A classificação foi realizada para a média dos erros de 2.023 séries de itens intermitentes da amostra. Neste primeiro grupo, foram consideradas as médias 
de erro DMAE, EMAE e PEEEAm, associadas por proximidade de resultados na classificação de otimização e desempenho dos modelos (Tabela 1).

Tabela 1 - Classificação das medidas de erros DMAE, EMAE e PEEEAm, para séries intermitentes (Primeiro Grupo), usando otimização.

\begin{tabular}{|c|c|c|c|c|c|}
\hline \multirow{2}{*}{$\begin{array}{c}\text { Tipo de } \\
\text { Otimização }\end{array}$} & Treino & \multicolumn{4}{|c|}{ Teste } \\
\cline { 2 - 6 } & Classificação & T+1 & T+3 & T+6 & Classificação \\
\cline { 2 - 6 } & 2,33 & 1,00 & 1,00 & 1,00 & 1,00 \\
DMA & 1,67 & 2,00 & 2,00 & 2,00 & 2,00 \\
DQM & 2,67 & 3,00 & 3,00 & 3,00 & 3,00 \\
TAM & 3,67 & 4,00 & 4,33 & 4,00 & 4,11 \\
TQM & 6,00 & 6,00 & 6,00 & 6,00 & 6,00 \\
$\alpha=0,1 ; p_{t}:$ naïve & 4,67 & 5,00 & 4,67 & 5,00 & 4,89 \\
$\alpha=0,1 ; p_{\mathrm{t}}$ : média & \multicolumn{5}{|c|}{} \\
\hline
\end{tabular}

Fonte: O autor, 2020.

O primeiro grupo de medidas apresentou melhor benefício para a otimização DMA na amostra de teste. Este resultado era esperado, uma vez que a função de perda utilizada tem origem na sua própria medida de erro (DMA). Para ilustrar o padrão de comportamento dos erros das otimizações no primeiro grupo, segue um exemplo para o horizonte de predição de 6 meses (Figura 10).

É possível avaliar também o benefício das otimizações em relação à utilização de parâmetros convencionais descritos na literatura. Mesmo a otimização de pior resultado (TQM) superou a utilização das constantes de suavização, recomendadas nas fontes de pesquisa $(\alpha=0,1 ; \beta=0,2$ para TSB, e o início dos intervalos entre demandas com método ingênuo (naïve) ou média destes intervalos). 
Figura 10 - Comportamento de medidas de erro nas otimizações do Primeiro Grupo para um horizonte de predição de 6 meses, usando desvios absolutos.
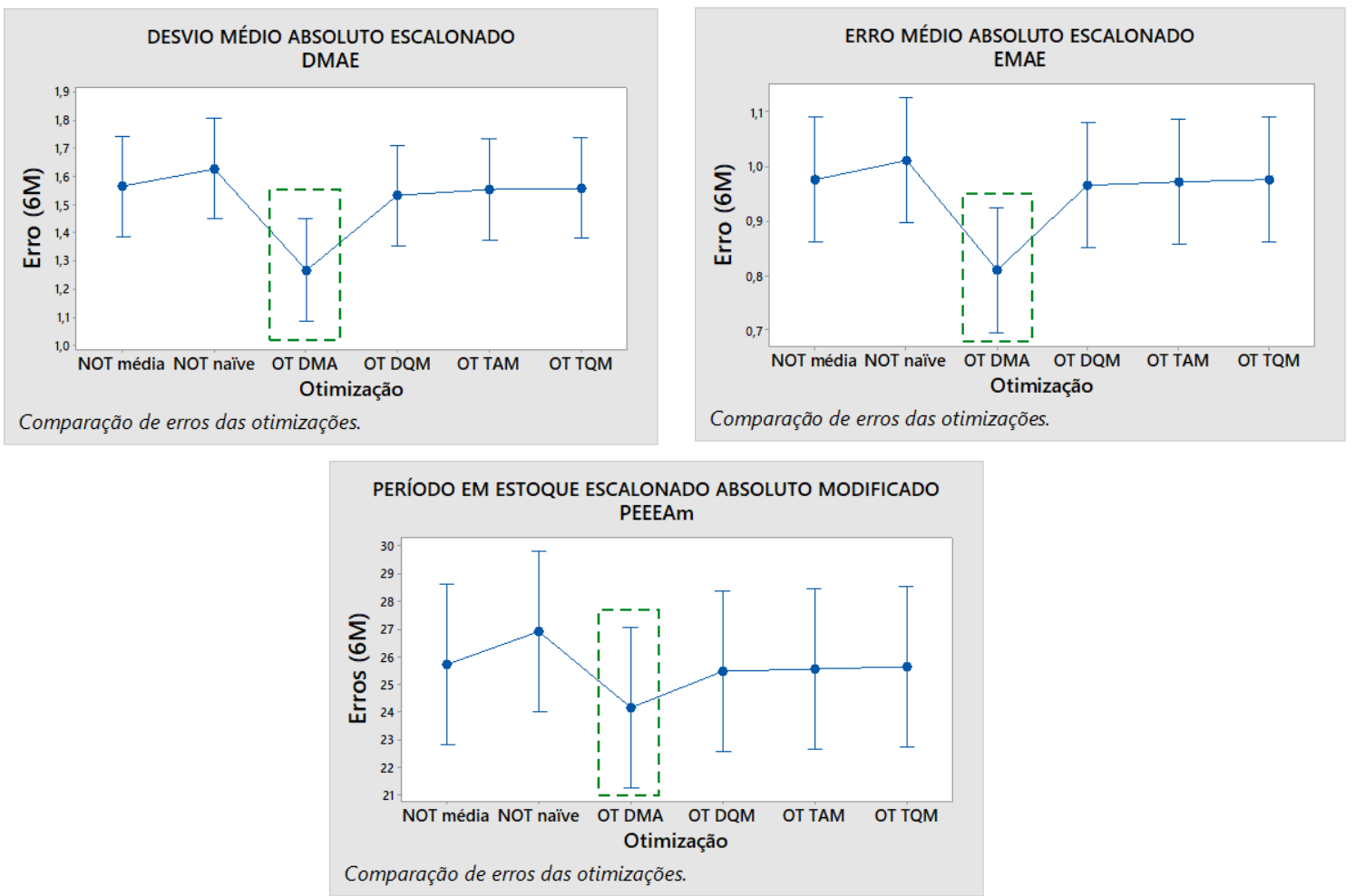

Fonte: O autor, 2020.

Os resultados da classificação das medidas de erros para séries de dados intermitentes, usando modelos de predição, encontram-se na Tabela 2.

Tabela 2 - Classificação das medidas de erros DMAE, EMAE e PEEEAm, para séries intermitentes (Primeiro Grupo), usando modelos de predição.

\begin{tabular}{|c|c|c|c|c|c|}
\hline \multirow{2}{*}{$\begin{array}{c}\text { Método de } \\
\text { Predição }\end{array}$} & Treino & \multicolumn{4}{|c|}{ Amostra } \\
\cline { 2 - 6 } & Classificação & T+1 & T+3 & T+6 & Classificação \\
\cline { 2 - 6 } & 5,33 & 7,00 & 7,00 & 7,00 & 7,00 \\
MMS3 & 5,00 & 4,00 & 4,00 & 4,33 & 4,11 \\
SBA & 6,33 & 6,00 & 6,00 & 6,00 & 6,00 \\
CROSTON & 4,67 & 3,00 & 3,00 & 3,00 & 3,00 \\
SBJ & 3,00 & 5,00 & 5,00 & 4,67 & 4,89 \\
SES & 3,33 & $\mathbf{1 , 0 0}$ & $\mathbf{1 , 0 0}$ & $\mathbf{1 , 0 0}$ & $\mathbf{1 , 0 0}$ \\
ZERO & 5,67 & 8,00 & 8,00 & 8,00 & 8,00 \\
NAÏVE & $\mathbf{2 , 6 7}$ & 2,00 & 2,00 & 2,00 & 2,00 \\
TSB & \multicolumn{4}{|c}{} \\
\hline
\end{tabular}

Fonte: $\mathrm{O}$ autor, 2020. 
As comparações de erros de predição para o horizonte de 6 meses para demanda intermitente (Primeiro Grupo) usando modelos, encontram-se na Figura 11.

Figura 11- Comparação dos erros nas predições do Primeiro Grupo de medidas para um horizonte de predição de 6 meses, usando os modelos.

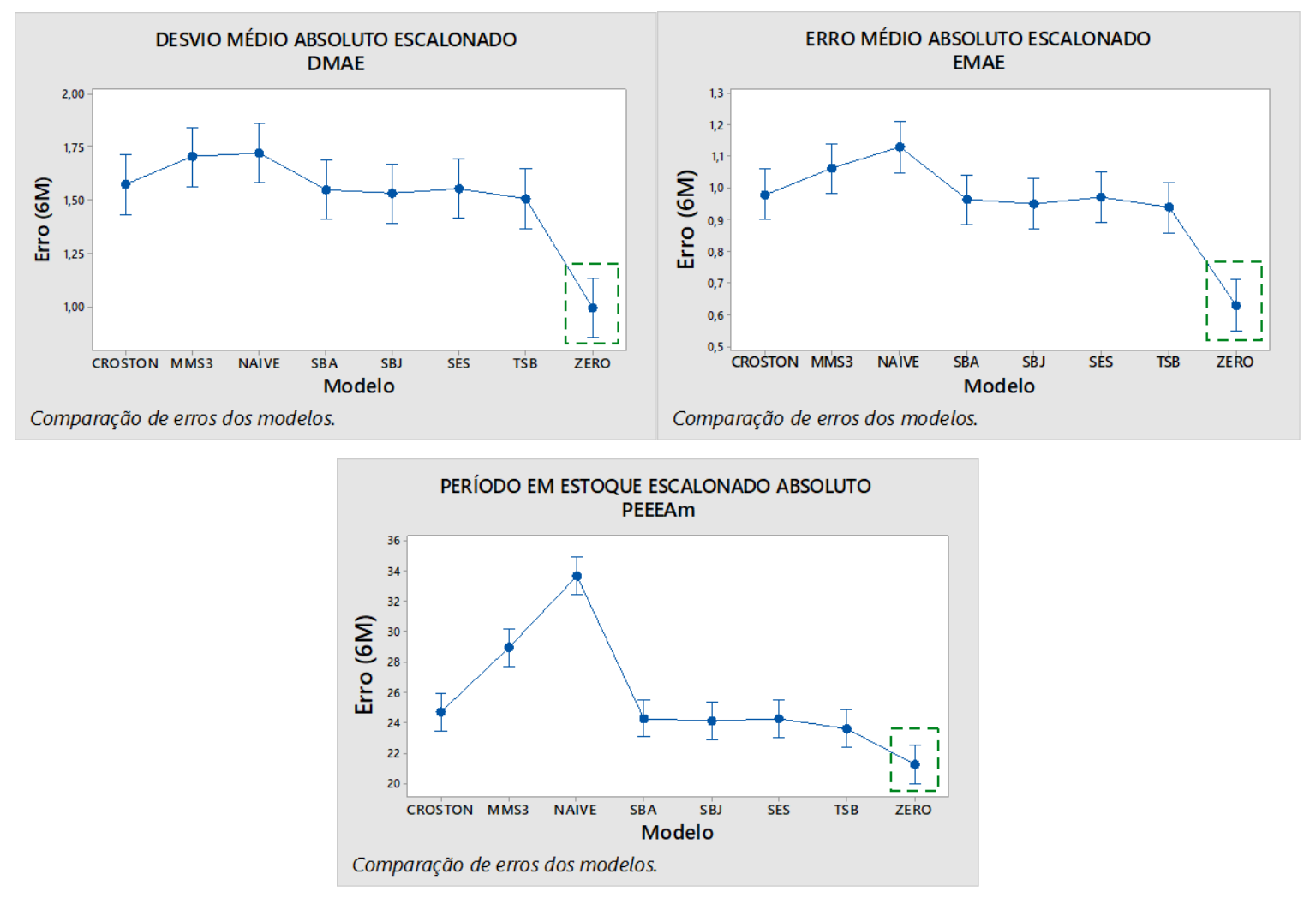

Fonte: O autor, 2020.

Observa-se que o método ZERO apresentou o melhor resultado de desempenho na amostra teste. Ao analisar este modelo (primeira colocação na classificação da amostra teste), verifica-se o melhor resultado em todos os cenários, quando comparado pelos desvios DMAE, EMAE e PEEEAm. Este resultado sugere uma semelhança com o descrito por Wällstrom e Segerstedt (2010), que destacam que em certas circunstâncias o DMA tende a subestimar a demanda, preferindo a predição zero. Portanto, a predição ficará mais distorcida, e um intervalo maior entre demanda tornará o DMA menos confiável. O intervalo maior entre demandas é o que define parcialmente a demanda intermitente.

O DMA não seria considerado adequado para uso em séries com demanda intermitente, o que estaria coincidindo também com as descobertas de Teunter e Duncan (2009), ao afirmarem que o DMA favorece a subestimação de métodos e 
que o DQM também o faz, mas não no mesmo grau. Também está de acordo com a descrição de Kourentzes (2014), que afirma que esta medida beneficia a predição zero de amostras com demandas intermitentes. Todos os métodos, exceto o método ZERO, tentam obter a correta predição por período, mas são punidos ao fazê-lo no cálculo de DMA.

As medidas de erro pelo DMA e EMAE são afetadas pelos mesmos benefícios da predição zero, o que pode ser evidenciado pelo resultado encontrado. Nas duas medidas, uma predição sendo zero todo o tempo seria a preferida, mesmo que esta predição seja inútil para o processo de manufatura. Isto é porque os valores dessas medidas de erro são absolutos, e procura-se a mediana de seus valores reais e não a média. A mediana de séries temporais de demanda intermitente é frequentemente igual a zero.

Os resultados de PEEEAm apresentaram a mesma tendência de resultados para a amostra teste, sendo por isto integrado ao Primeiro Grupo. Esta medida também é apresentada em valor absoluto. Neste caso, houve uma discordância com descrito por Kourentzes (2014), que seria uma vantagem desta medida a penalização adequada das predições de demanda zero.

Foi observado também que o método CROSTON mostrou desempenho inferior em relação a seus sucessores, SBA e SBJ. Isto era esperado, conforme pesquisas de Syntetos e Boylan (2005) e de Shale, Boylan e Johnston (2006), para demanda intermitente. O método TSB ficou na segunda posição entre todos os modelos, e os métodos MMS3 e NAÏVE apresentaram os maiores erros.

1.11.2 Resultado do Segundo Grupo de medidas de erro para demanda intermitente

No Segundo Grupo de medidas de erro, associados por proximidade nos resultados na classificação de otimização e desempenho dos modelos, foram consideradas as medidas de desvio DQME, TAME e TQME. Mesmo o DQME sendo pontual (não uma medida de taxa acumulada), seus resultados se aproximaram aos das medidas TAME e TQME, e por isto foi associado a este grupo. Os resultados estão simplificados na forma de classificação por posição, sendo a menor posição na classificação equivalente ao melhor desempenho, na Tabela 3. 
Tabela 3 - Classificação das medidas de erros DQME, TAME e TQME, para séries intermitentes (Segundo Grupo), usando otimização.

\begin{tabular}{|c|c|c|c|c|c|}
\hline \multirow{2}{*}{$\begin{array}{c}\text { Tipo de } \\
\text { Otimização }\end{array}$} & Treino & \multicolumn{4}{|c|}{ Amostra } \\
\cline { 2 - 6 } & Classificação & T+1 & T+3 & T+6 & Classificação \\
\hline \multirow{yyyyyy}{*}{ DMA } & 5,33 & 6,00 & 6,00 & 6,00 & 6,00 \\
DQM & $\mathbf{2 , 6 7}$ & 4,33 & 4,67 & 4,67 & 4,56 \\
TAM & $\mathbf{2 , 6 7}$ & $\mathbf{1 , 0 0}$ & $\mathbf{1 , 0 0}$ & $\mathbf{1 , 0 0}$ & $\mathbf{1 , 0 0}$ \\
TQM & $\mathbf{1 , 6 7}$ & $\mathbf{2 , 0 0}$ & $\mathbf{2 , 0 0}$ & $\mathbf{2 , 0 0}$ & $\mathbf{2 , 0 0}$ \\
$\alpha=0,1 ;$ pt: naïve & 5,67 & 4,67 & 4,33 & 4,33 & 4,44 \\
$\alpha=0,1 ;$ pt: média & 3,00 & 3,00 & 3,00 & 3,00 & 3,00 \\
\hline
\end{tabular}

Fonte: O autor, 2020.

Neste segundo grupo, os melhores desempenhos foram obtidos com a otimização das taxas TAM e TQM, tanto na amostra de treino quanto na de teste. Este resultado era esperado, porque as funções de perda destas otimizações têm origem nas próprias medidas de erros alternativas (KOURENTZES, 2014). Um exemplo do padrão de comportamento dos erros das otimizações deste Segundo Grupo é apresentado na Figura 12, para o horizonte de predição de 6 meses, usando otimização.

Uma diferença do Segundo Grupo em relação ao Primeiro Grupo é a inversão do benefício do DMA que, no Segundo Grupo, apresenta pior desempenho. Neste caso, a otimização por DMA não supera, nem mesmo, a utilização dos parâmetros convencionais citados na literatura. 
Figura 12 - Comportamento de medidas de erro nas otimizações do Segundo Grupo para um horizonte de predição de 6 meses, usando desvios quadráticos e/ou acumulativos.
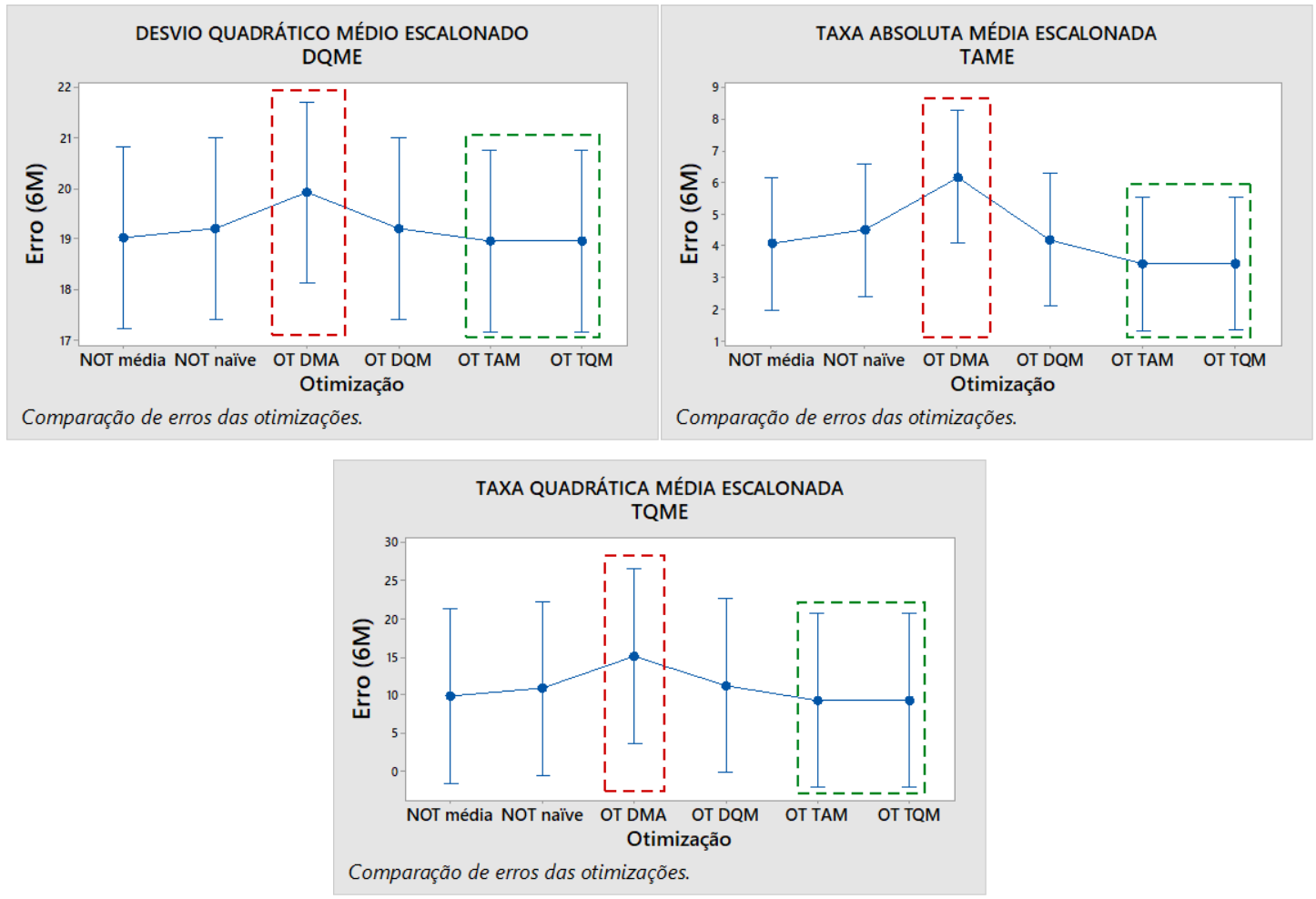

Fonte: O autor, 2020.

Os resultados da classificação das medidas de erros para séries de dados intermitentes, no Segundo Grupo, usando modelos de predição, encontram-se na Tabela 4 e a comparação de erros de predição para o horizonte de 6 meses é apresentada na Figura 13.

Observa-se na Tabela 4, que os melhores resultados de desempenho para a amostra teste do Segundo Grupo, foram para os métodos SBA e SBJ. Este foi o resultado consolidado das opções de otimização que, individualmente, poderiam apresentar desempenho diferentes. Os métodos NAÏVE, MMS3 e ZERO apresentaram os piores desempenhos neste $2^{\circ}$ grupo.

A mesma tendência observada na Tabela 4 é confirmada na Figura 13, para as três medidas de erro DQME, TAME e TQME, em todas as otimizações. Observase também que o método CROSTON apresenta resultados inferiores aos dos seus métodos sucessores SBA e SBJ. Os resultados detalhados de cada simulação de otimização dos modelos de predição estão nos Apêndices A e B. 
Tabela 4 - Classificação das medidas de erros DQME, TAME e TQME, para séries intermitentes (Segundo Grupo), usando modelos de predição.

\begin{tabular}{|c|c|c|c|c|c|}
\hline \multirow{2}{*}{$\begin{array}{c}\text { Método de } \\
\text { Predição }\end{array}$} & \multicolumn{5}{|c|}{ Amostra } \\
\cline { 2 - 6 } & Treino & \multicolumn{4}{|c|}{ Teste } \\
\cline { 2 - 6 } & Classificação & $\mathbf{T + 1}$ & $\mathbf{T + 3}$ & $\mathbf{T + 6}$ & Classificação \\
\hline MMS3 & $\mathbf{6 , 6 7}$ & $\mathbf{7 , 0 0}$ & $\mathbf{7 , 0 0}$ & 7,00 & $\mathbf{7 , 0 0}$ \\
SBA & $\mathbf{1 , 3 3}$ & $\mathbf{1 , 3 3}$ & $\mathbf{1 , 6 7}$ & $\mathbf{2 , 0 0}$ & $\mathbf{1 , 6 7}$ \\
CROSTON & 3,33 & 3,33 & 3,33 & 3,67 & 3,44 \\
SBJ & $\mathbf{2 , 6 7}$ & $\mathbf{2 , 3 3}$ & $\mathbf{2 , 0 0}$ & $\mathbf{2 , 0 0}$ & $\mathbf{2 , 1 1}$ \\
SES & 4,67 & 4,00 & 4,00 & 4,00 & 4,00 \\
ZERO & 6,33 & 6,00 & 6,00 & 6,00 & 6,00 \\
NAÏVE & 8,00 & 8,00 & 8,00 & 8,00 & 8,00 \\
TSB & 3,00 & 4,00 & 4,00 & 3,33 & 3,78 \\
\hline
\end{tabular}

Fonte: O autor, 2020.

Figura 13 - Comparação dos erros nas predições do Segundo Grupo de medidas para um horizonte de predição de 6 meses, usando os modelos.
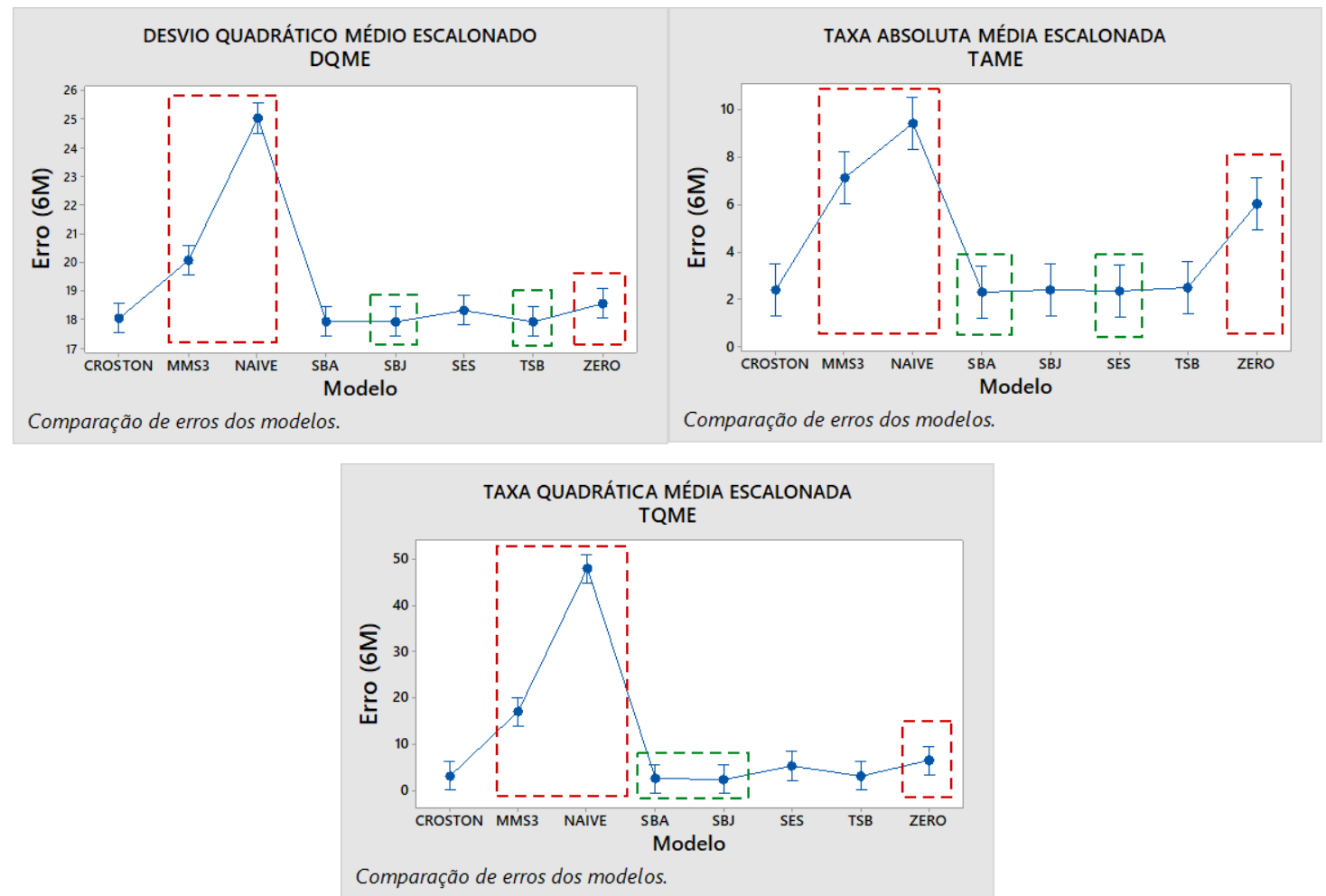

Fonte: O autor, 2020.

Como consolidação destas análises, sugere-se que a escolha das medidas de erro pode influenciar no desempenho das otimizações e dos modelos. Os dois 
agrupamentos definidos para simplificação do resultado da pesquisa, descrevem padrões de comportamentos distintos quanto aos resultados da otimização e consequentemente quanto ao desempenho de cada método.

\subsection{Comparação dos resultados da otimização de demandas}

1.12.1 Comparação da otimização para demanda de alto giro

A validação dos resultados anteriormente apresentados foi realizada por meio de comparação para enquadrar-se no foco da pesquisa. Este procedimento foi realizado avaliando-se uma parcela menor de itens com demandas não intermitentes da amostra (380 itens), por meio de novas simulações de predição para séries de demanda com alto giro (suaves e erráticas).

Foi determinado o desempenho das predições para os métodos, cujos resultados estão contidos na Tabela 5 e uma comparação dos erros de predição para o horizonte de 6 meses é apresentada na Figura 14.

Tabela 5 - Classificação das medidas de erros DMAE, EMAE e PEEEAm, para séries de alto giro - suaves e erráticas (Primeiro Grupo), usando modelos de predição.

\begin{tabular}{|c|c|c|c|c|c|}
\hline \multirow{2}{*}{$\begin{array}{c}\text { Método de } \\
\text { Predição }\end{array}$} & \multicolumn{5}{|c|}{ Amostra } \\
\cline { 2 - 6 } & Treino & \multicolumn{4}{|c|}{ Teste } \\
\cline { 2 - 6 } & Classificação & $\mathbf{T + 1}$ & $\mathbf{T + 3}$ & $\mathbf{T + 6}$ & Classificação \\
\hline MMS3 & 4,67 & $\mathbf{1 , 0 0}$ & $\mathbf{1 , 2 5}$ & $\mathbf{1 , 7 5}$ & $\mathbf{1 , 4 2}$ \\
SBA & 4,33 & 5,67 & 5,25 & 5,25 & 5,42 \\
CROSTON & 5,00 & 7,00 & 6,25 & 6,25 & 6,50 \\
SBJ & 4,67 & 4,67 & 4,25 & 4,25 & 4,42 \\
SES & $\mathbf{1 , 6 7}$ & 3,00 & 3,25 & 3,00 & 3,17 \\
ZERO & 8,00 & 8,00 & 8,00 & 8,00 & $\mathbf{8 , 0 0}$ \\
NAÏVE & 5,00 & 4,67 & 5,50 & 5,50 & 4,92 \\
TSB & $\mathbf{2 , 6 7}$ & $\mathbf{2 , 0 0}$ & $\mathbf{2 , 2 5}$ & $\mathbf{2 , 0 0}$ & $\mathbf{2 , 1 7}$ \\
\hline
\end{tabular}

Fonte: O autor, 2020. 
Figura 14- Comparação dos erros nas predições do Primeiro Grupo de medidas para um horizonte de predição de 6 meses para demanda de alto giro, usando os modelos.
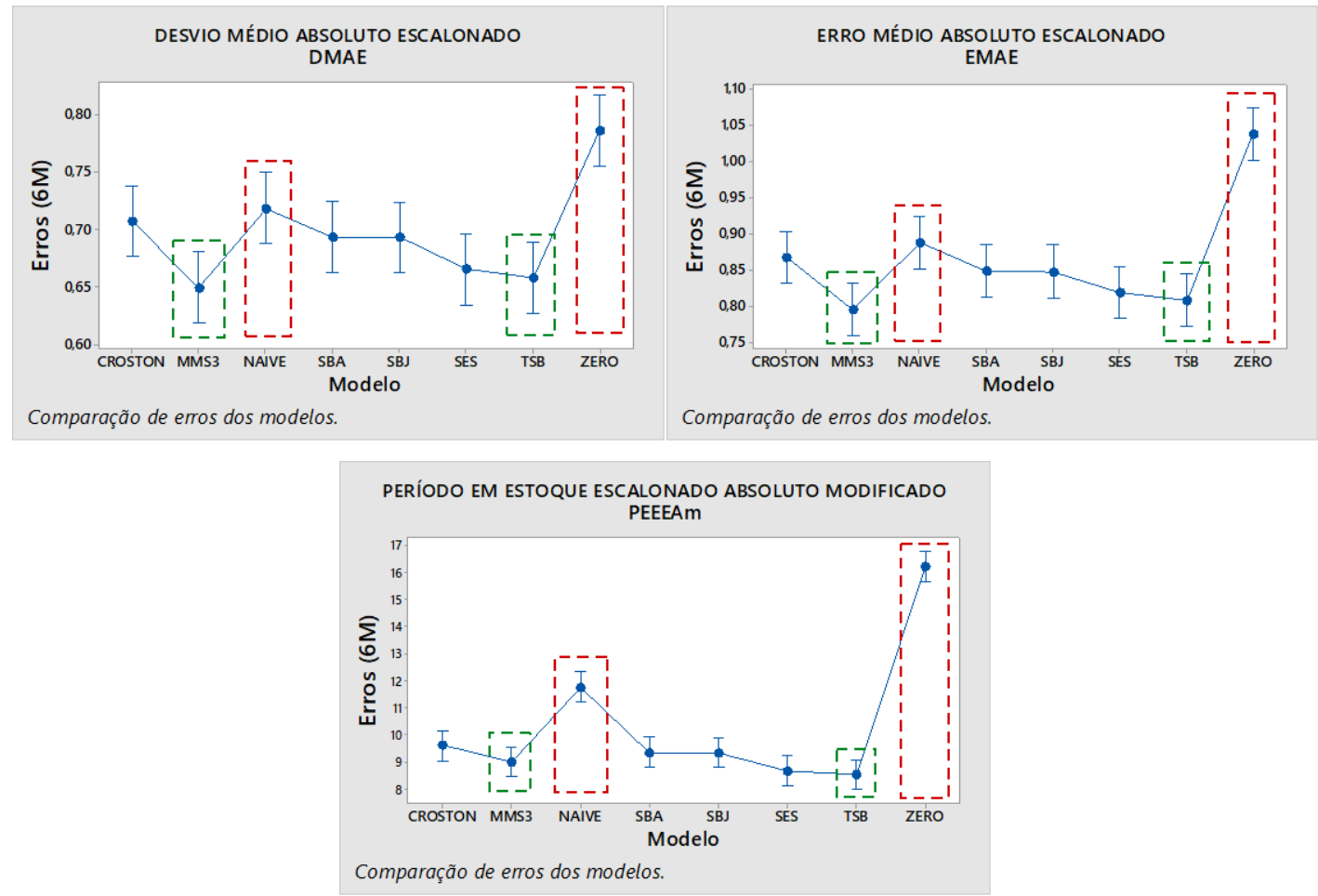

Fonte: O autor, 2020.

Os mesmos grupos de medidas foram utilizados, e para o primeiro grupo, os métodos MMS3 e TSB apresentaram melhor desempenho. Ao contrário dos itens de demanda intermitente, observa-se que o método ZERO apresentou o pior desempenho, ficando na oitava posição em todas as combinações de medidas de erro e otimizações da amostra de treino e de teste. Este resultado era esperado, uma vez que a maior frequência de demanda, implica em maior erro quando a predição é zero, para todos os cenários. Ao contrário, o método MMS3 (de 3 meses) é beneficiado e, apesar de simples, pode ser usual em séries não intermitentes para este grupo de medidas.

Para o Segundo Grupo da série de demanda de alto giro, foi determinado o desempenho dos modelos de predição usando os métodos apresentados, cujos resultados estão contidos na Tabela 6 e uma comparação dos erros de predição para o horizonte de 6 meses é apresentada na Figura 15.

Observa-se que, para itens com demanda de alto giro, os métodos destacados foram os de SES, CROSTON e SBA. O método de SES apresentou o melhor resultado e o método SBA ficou na segunda colocação para itens com 
demanda de alto giro, enquanto havia sido classificado na primeira posição para itens com demanda intermitente.

Tabela 6 - Classificação das medidas de erros DQME, TAME e TQME, para séries de alto giro - suaves e erráticas (Segundo Grupo), usando modelos de predição.

\begin{tabular}{|c|c|c|c|c|c|}
\hline \multirow{2}{*}{$\begin{array}{c}\text { Método de } \\
\text { Predição }\end{array}$} & \multicolumn{5}{|c|}{ Amostra } \\
\cline { 2 - 6 } & Treino & \multicolumn{4}{|c|}{ Teste } \\
\cline { 2 - 6 } & Classificação & $\mathbf{T + 1}$ & $\mathbf{T + 3}$ & $\mathbf{T + 6}$ & Classificação \\
\hline MMS3 & $\mathbf{6 , 0 0}$ & 5,00 & 6,00 & 6,00 & 5,67 \\
SBA & 3,00 & 3,33 & 3,00 & $\mathbf{2 , 3 3}$ & $\mathbf{2 , 8 9}$ \\
CROSTON & $\mathbf{2 , 6 7}$ & $\mathbf{3 , 0 0}$ & $\mathbf{2 , 6 7}$ & 3,00 & $\mathbf{2 , 8 9}$ \\
SBJ & $\mathbf{4 , 0 0}$ & $\mathbf{3 , 6 7}$ & $\mathbf{3 , 3 3}$ & $\mathbf{3 , 6 7}$ & $\mathbf{3 , 5 6}$ \\
SES & $\mathbf{2 , 3 3}$ & $\mathbf{2 , 3 3}$ & $\mathbf{2 , 3 3}$ & $\mathbf{2 , 3 3}$ & $\mathbf{2 , 3 3}$ \\
ZERO & $\mathbf{3 , 0 0}$ & $\mathbf{8 , 0 0}$ & $\mathbf{8 , 0 0}$ & $\mathbf{8 , 0 0}$ & $\mathbf{8 , 0 0}$ \\
NAÏVE & 7,00 & 7,00 & 7,00 & 7,00 & $\mathbf{7 , 0 0}$ \\
TSB & 3,00 & 3,67 & 3,67 & 3,67 & 3,67 \\
\hline
\end{tabular}

Fonte: O autor, 2020.

Figura 15- Comparação dos erros nas predições do Segundo Grupo de medidas para um horizonte de predição de 6 meses para demanda de alto giro, usando os modelos.

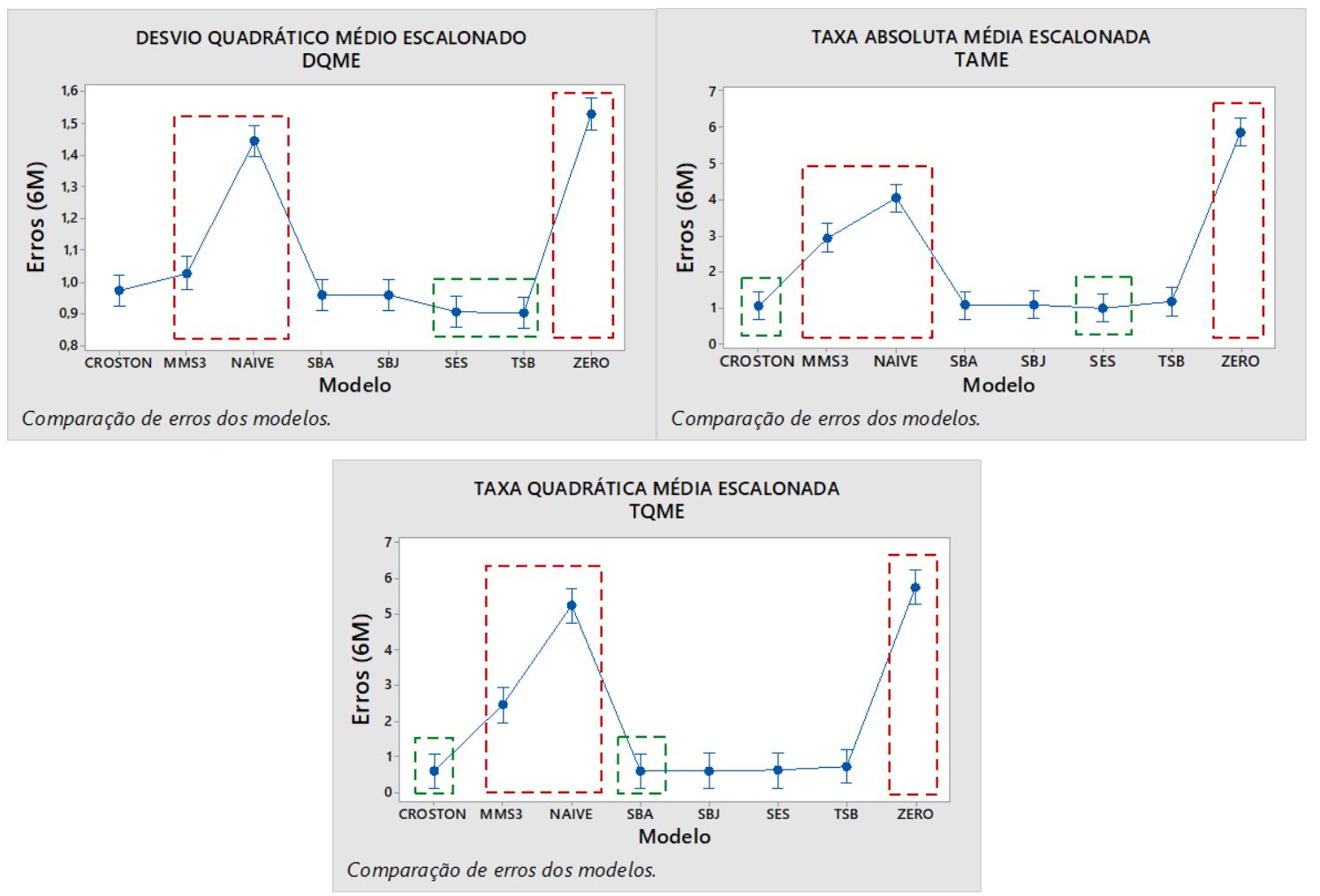

Fonte: O autor, 2020. 
O método de CROSTON apresentou o mesmo benefício do SBA, o que sugere a aplicação deste método para itens de natureza suave. Este fato pode indicar a concordância com o resultado apresentado em Syntetos e Boylan (2005), que recomendaram o método de CROSTON para séries temporais suaves e SBA para as demais.

O método TSB destacou-se por apresentar a melhor flexibilidade geral, ficando bem posicionado, tanto para itens com demanda intermitente quanto para itens com demanda de alto giro, nas diferentes configurações dos grupos. Mesmo não alcançando a primeira colocação, demonstrou adaptabilidade aos grupos de medidas e natureza da amostra. Como descrito anteriormente, este método é interessante para objetivos de inventário, pois trata a obsolescência. O método possui a característica de atualizar o valor da estimação de probabilidade de procura continuamente ao longo de diversos períodos. Isto permite o método TSB predizer uma diminuição gradual de demanda, a ponto da demanda se tornar zero para itens descontinuados, evitando-se excessos no inventário. O detalhamento de cada simulação de otimização dos modelos de predição dos itens com demanda de alto giro, encontram-se nos Apêndices C e D.

1.13 Proposta de otimização do processo de predição a partir de cada medida de erro especificada

\subsubsection{Sugestões}

Os resultados obtidos neste livro sugerem que, conforme as medidas de erros sejam escolhidas, o comportamento dos processos de otimização e do desempenho dos modelos se modificam. Desta forma, para cada aplicação, cabe a medida de erro e modelo de predição mais adequado ao trabalho.

Os resultados também sugerem uma concordância com Wallström e Segerstedt (2010), os quais relataram que, quando um erro de predição é mensurado, não é o método que é medido, mas a combinação de como este método responde ao erro. As relações entre diferentes erros podem variar de acordo com o método aplicado, o valor inicial, as constantes de suavização, as medidas de variância e viés. Também há concordância com Lee e Everett (1986), que chegaram à conclusão de que, em uma situação de manufatura, uma superestimação é 
preferida, devido à necessidade de menos configurações e, portanto, menor custo. Quando os objetivos de inventário são considerados, uma subestimação pode ser melhor, se o estoque de segurança for baseado no desempenho da variação. As sugestões, a partir das evidências encontradas neste trabalho, são resumidas no Quadro 2.

Quadro 2 - Resumo de sugestões para demanda intermitente.

\begin{tabular}{|c|c|c|c|c|c|}
\hline \multirow{2}{*}{$\begin{array}{c}\text { Natureza da } \\
\text { demanda }\end{array}$} & \multirow[b]{2}{*}{ Objetivo } & \multicolumn{4}{|c|}{ Sugestões para demanda intermitente } \\
\hline & & $\begin{array}{l}\text { Medidas } \\
\text { de erro }\end{array}$ & Otimização & Método & Observação \\
\hline \multirow[t]{2}{*}{ Intermitente } & $\begin{array}{c}\text { Controle de } \\
\text { inventário } \\
\text { combinado } \\
\text { ao } \\
\text { cronograma } \\
\text { de } \\
\text { produção }\end{array}$ & $\begin{array}{l}\text { DQME } \\
\text { TAME } \\
\text { TQME }\end{array}$ & $\begin{array}{l}\text { TAM } \\
\text { TQM }\end{array}$ & $\begin{array}{l}\text { SBA } \\
\text { SBJ }\end{array}$ & $\begin{array}{l}\text { Reduz a } \\
\text { tendência de } \\
\text { predições zero, } \\
\text { quando ocorre } \\
\text { otimização. } \\
\text { Necessário o } \\
\text { acompanhamento } \\
\text { posterior de itens } \\
\text { obsoletos. }\end{array}$ \\
\hline & $\begin{array}{c}\text { Ajuste } \\
\text { automático } \\
\text { de predição } \\
\text { para itens } \\
\text { obsoletos }\end{array}$ & $\begin{array}{l}\text { DQME } \\
\text { TAME } \\
\text { TQME }\end{array}$ & $\begin{array}{l}\text { TAM } \\
\text { TQM }\end{array}$ & TSB & $\begin{array}{l}\text { Predição se } \\
\text { ajusta } \\
\text { automaticamente } \\
\text { para itens } \\
\text { obsoletos até a } \\
\text { completa } \\
\text { descontinuidade. }\end{array}$ \\
\hline
\end{tabular}

Fonte: O autor, 2020

Para os itens com demanda de alto giro não foram apresentadas sugestões, pois existem outros modelos apropriados para esta natureza de demanda que não foram discutidos nesta pesquisa. A comparação dos resultados ocorreu apenas para validar o comportamento dos erros da demanda intermitente. É importante destacar que as sugestões acompanham a amostra estudada, e para novos conjuntos de dados fazem-se necessárias análises específicas.

\subsubsection{Análise de parâmetros de suavização para demanda intermitente}

Posteriormente, foram avaliados os efeitos das otimizações nas médias dos parâmetros de suavização, para analisar o efeito da otimização sobre estas 
constantes. Os resultados destas avaliações estão apresentados nos gráficos das Figuras 16, 17, 18 e 19.

Figura 16 - Otimização usando o DMA (média, $\left.\alpha \_1, \alpha \_2 ; \beta \_2-T S B\right)$.

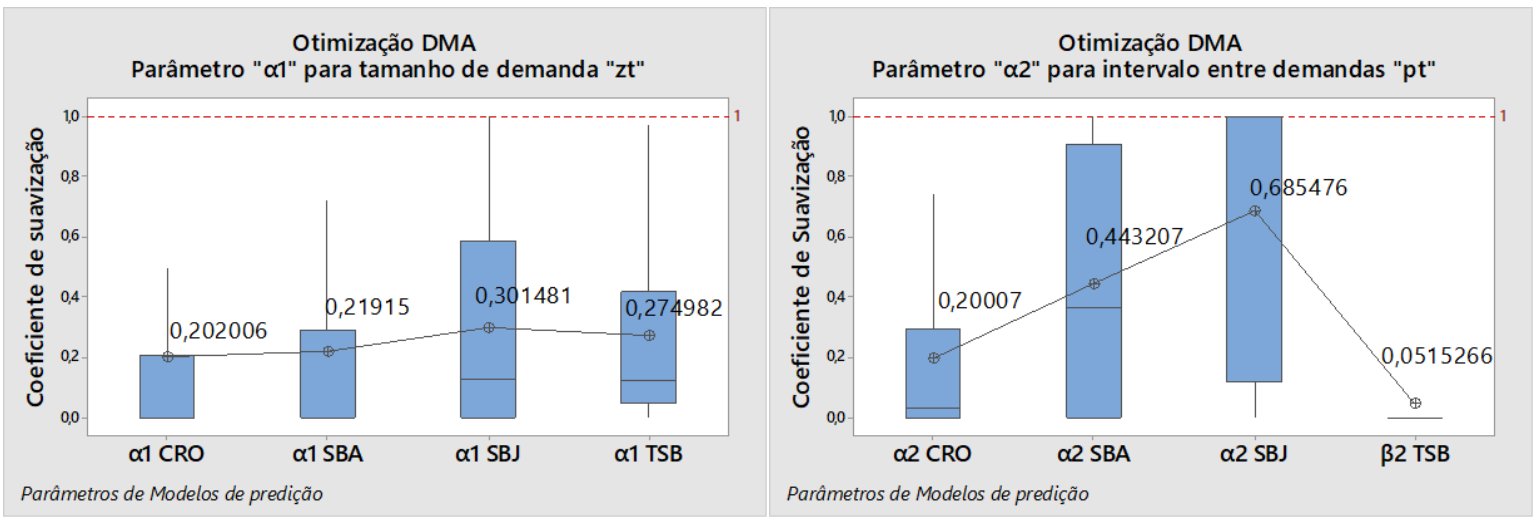

Fonte: O autor, 2020

Figura 17 - Otimização usando o DQM (média, a_1, a_2; $\beta$ _2-TSB).

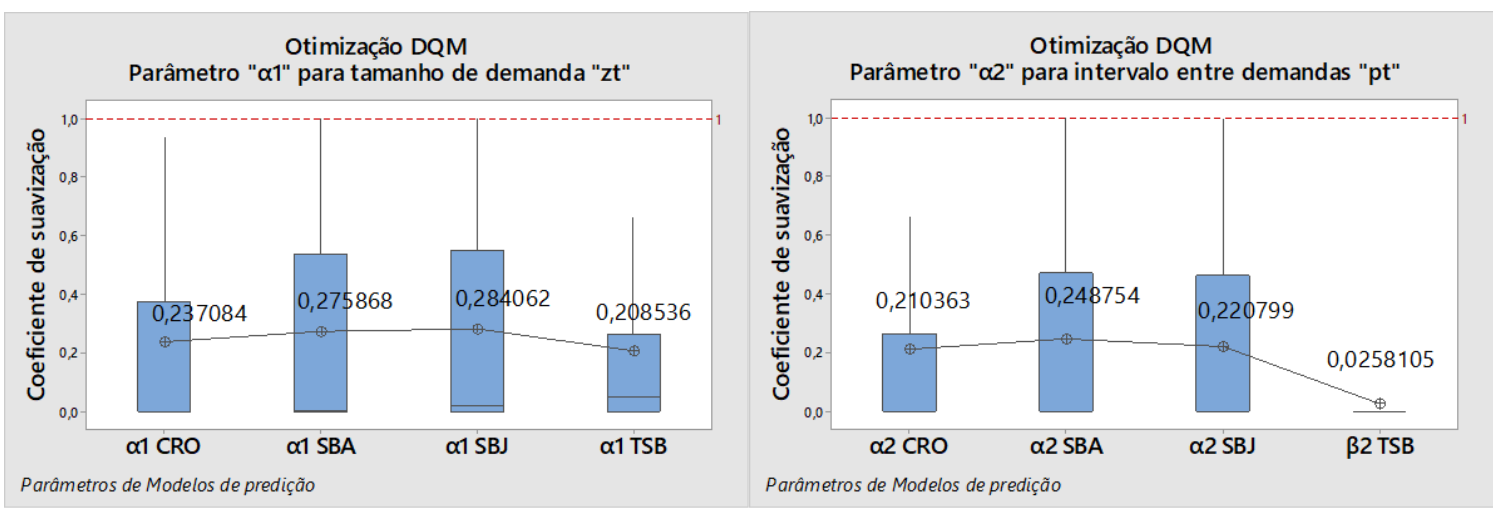

Fonte: O autor, 2020.

Figura 18 - Otimização usando o TAM (média, $\left.\alpha \_1, \alpha \_2 ; \beta \_2-T S B\right)$.

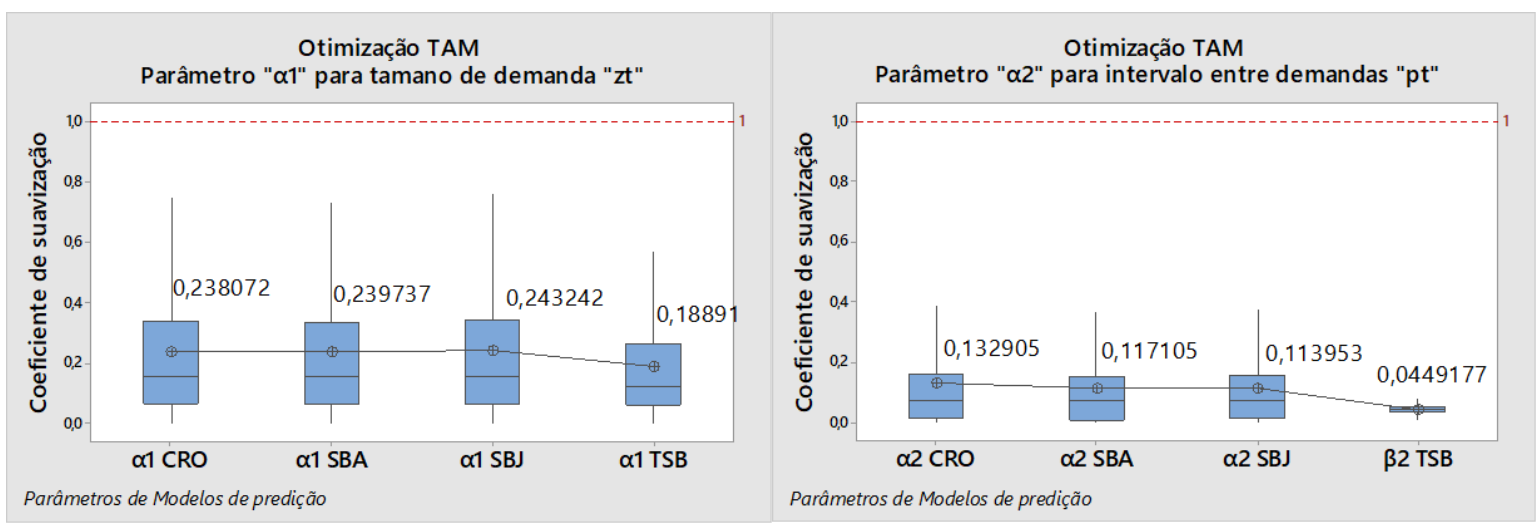

Fonte: O autor, 2020. 
Figura 19 - Otimização usando o TQM (média, $\left.\alpha \_1, \alpha \_2 ; \beta \_2-T S B\right)$.

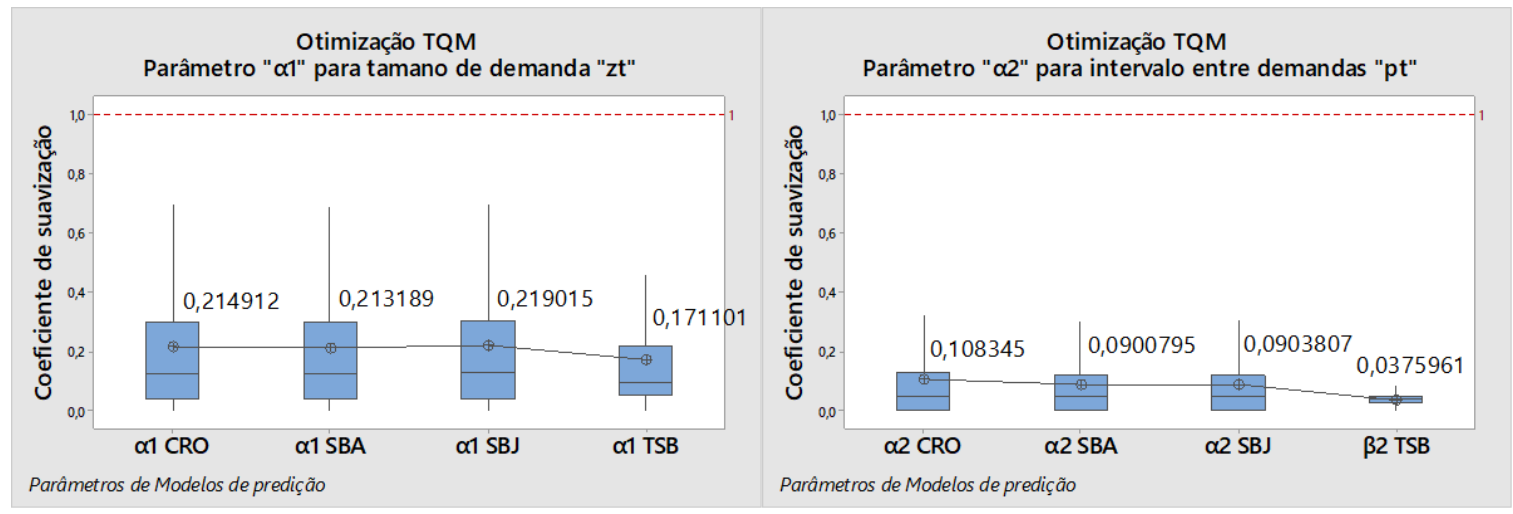

Fonte: O autor, 2020.

Esta análise foi importante para identificar o comportamento irregular das constantes de suavização para a otimização por meio do DMA. A principal variação entre o DMA e as demais otimizações, ocorre para o parâmetro $\alpha_{2}$, a constante de suavização do intervalo entre demandas. Os valores destes parâmetros são bem menores e nivelados, para as otimizações por meio de TAM e TQM. Para o parâmetro $\alpha_{1}$, a constante de suavização do tamanho da demanda diferente de zero, os valores resultantes das otimizações por meio da TAM e TQM também são mais regulares. Um menor valor da constante de suavização combinado com um menor valor inicial, aumenta a possibilidade de menor erro para predições de demanda intermitente (WALLSTRÖM; SEGERSTEDT, 2010).

Estes resultados estão em acordo com Kourentzes (2014), que também afirma o benefício das otimizações em relação à utilização de parâmetros Ad hoc. O objetivo de encontrar uma constante de suavização adequada é reduzir o tamanho do estoque de segurança afetado pelo erro de predição. 


\section{CONCLUSÕES}

A predição de itens com demanda intermitente próximas ao valor real é um dos objetivos para uma boa gestão de inventário. Com este objetivo, algumas técnicas de predição descritas na literatura foram testadas e avaliadas quanto ao desempenho. Para isto, foi utilizada uma amostragem real de peças de reposição do setor de manutenção na indústria de mineração. Procurou-se mensurar e identificar o padrão de comportamento dos erros, a partir de dados reais, conforme medidas e métodos utilizados. Uma análise das otimizações e seu efeito nos parâmetros das funções de perda também foi realizada.

Os resultados encontrados sugerem que a escolha da medida de erro interfere no resultado de desempenho, tanto das otimizações quanto dos modelos. Esta constatação foi considerada com base na avaliação da classificação dos erros em dois grupos distintos de medidas. Estes grupos foram definidos por semelhança de resultado na classificação e padrões de comportamento dos erros.

Como o objetivo do trabalho foi a avaliação da predição de peças de reposição com demanda intermitente, em que não faz sentido predições zero, recomenda-se as medidas de acurácia do segundo grupo (TAME, TQME ou DQME). O melhor benefício ocorreu para as otimizações TAM e TQM e, consequentemente, com tendência à escolha dos modelos SBA e SBJ, os quais entende-se que sejam mais adequados ao processo de controle de inventário combinado ao cronograma de produção.

Para o controle automático de itens obsoletos do inventário sugere-se o método TSB, com resultados intermediários, porém mais regulares nos diversos cenários. Para os itens de natureza suave, com maior giro de demanda, analisados para comparação, os métodos de SES, CROSTON e SBA apresentaram melhor benefício. Destaca-se que estes resultados estão relacionados apenas à natureza da amostra da pesquisa, e novos estudos são necessários para outros conjuntos de dados.

Com base nas análises, concluiu-se que não se pode afirmar quais medidas, modelos e tipos de otimização são melhores, sem que antes seja definido o objetivo, porque quando um erro de predição é mensurado, não é o método que é medido, mas a combinação de como este método responde à medida de erro. Portanto, a 
definição do objetivo da predição é o primeiro passo antes da escolha da medida de erro adequada para posterior seleção do modelo e da técnica de otimização.

Os resultados desta pesquisa sugerem que o tema abordado ainda necessita ser explorado, haja vista que os sistemas de predição disponíveis nos programas (ou plataformas) computacionais empresariais não se aplicam diretamente à demanda intermitente e, quando existem, podem não ser bem compreendidos e aplicados. Como resultado, espera-se contribuir para melhor gestão de demanda intermitente, tanto para processos de manufatura quanto para objetivos específicos de inventário.

Uma limitação deste trabalho foi a análise do reflexo no nível de serviço e o custo de inventário, haja vista que, pela indisponibilidade de dados de inventário e seus custos, optou-se pela abordagem da acurácia de predições.

\subsection{Sugestões para trabalhos futuros}

Para novas pesquisas, sugere-se procurar por métodos que detectem outras situações em que medidas de erros de predição não sejam adequadas para um determinado método.

Buscar formas de medir a sensibilidade dos erros em relação às alterações das constantes de suavização e valores iniciais também seriam interessantes.

Aplicações de redes neurais e aprendizado de máquina (machine learning) poderiam ser úteis para viabilizar modelos com constantes de suavização dinâmicas ao longo das séries, que tornariam viáveis em técnicas como agregação, geração empírica de distribuições de dados (bootstrapping), sistema Neuro-Fuzzy, dentre outras.

Para avaliação de desempenho de modelos e/ou técnicas de predição, sugerem-se pesquisas considerando indicadores de desempenho de nível de serviço e custo de inventário. 


\section{REFERÊNCIAS}

CROSTON, J. D. Forecasting and stock control for intermittent demands. Journal of the Operational Research Society, v. 23, n. 3, p. 289-303, 1972.

HADDAD, M. Estudo comparativo entre sínteses de controle robusto paramétrico. 2001. 104 f. Dissertação (Mestrado em Engenharia Elétrica) - Instituto Militar de Engenharia, Rio de Janeiro, 2001.

HYNDMAN, R. J.; ATHANASAUPOLOS, G. Forecasting: principles and pratice. Disponível em: https://www.otexts.org/fpp2. Acesso em:Nov. 2019.

HYNDMAN, R. J.; KOEHLER, A. B. Another look at measures of forecast accuracy. International Journal of Forecasting, v. 22, n. 4, p. 679-688, 2006.

JOHNSTON, F. R.; BOYLAN, J. E.; SHALE, E. A. An examination of the size of orders from customers, their characterisation and the implications for inventory control of slow moving items. Journal of the Operational Research Society, v. 54, n. 8, p. 833-837, 2003.

KOURENTZES, N. On intermittent demand model optimisation and selection. International Journal of Production Economics, v. 156, p. 180-190, 2014.

LEE, T. S.; ADAM, E. E. Forecasting error evaluation in Material Requirements Planning (MRP) production-inventory systems. Management Science, v. 32, n. 9, p. 1186-1205, 1986.

MAKRIDAKIS, S.; WHEELRIGHT, S. C.; HYNDMAN, R. J. Forecasting:methods and aplications. 3. ed. New York: Jonh Wiley, 1998.

MORETTIN, P. A.; TOLOI, C. M. C. Análise de séries temporais. 2. ed. São Paulo: Edgard Blücher, 2006.

PELLEGRINI, F. R.; FOGLIATTO, F. S. Passos para implantação de sistemas de previsão de demanda: técnicas e estudo de caso. Production, v. 11, n. 1, p. 43-64, 2001.

PETROPOULOS, F.; NIKOLOPOULOS, K. Optimizing theta model for monthly data. In: ICAART 2013 - PROCEEDINGS OF THE FIFTHINTERNATIONAL CONFERENCE ON AGENTS AND ARTIFICIAL INTELLIGENCE, 1., 2013, Barcelona. Anais [...]. Barcelona, p. 190-195.

SANI, B.; KINGSMAN, B. G. Selecting the best periodic inventory controldemand forecasting methods for low demand items. Journal of the Operational Research Society, v. 48, n. 7, p. 700-713, 1997.

SANJOY, K. P. Determination of exponential smoothing constant to minimize mean square error and mean absolute deviation. Global Journal of Research in Engineering, v. 11, n. 3, p. 30-34, 2011.

SHALE, E. A.; BOYLAN, J. E.; JOHNSTON, F. R. Forecasting for intermittent demand: the estimation of an unbiased average. Journal of the Operational Research Society, v. 57, n. 5, p. 588-592, 2006.

SILVA, E. C. N. PMR 5215 - Otimização Aplicada ao Projeto de Sistemas Mecânicos.São Paulo: Departamento de Engenharia Mecatrônica e Sistemas Mecânicos, 
Escola Politécnica da USP, 2003.Apostila.

SYNTETOS, A. A.; BOYLAN, J. E. On the bias of intermittent demand estimates. International Journal of Production Economics, v. 71, n. 1-3, p. 457-466, 2001.

SYNTETOS, A. A.; BOYLAN, J. E. The accuracy of intermittent demand estimates. International Journal of Forecasting, v. 21, n. 2, p. 303-314, 2005.

SYNTETOS, A. A.; BOYLAN, J. E. On the stock control performance of intermittent demand estimators. International Journal of Production Economics, v. 103, n. 1, p. 36-47, 2006.

SYNTETOS, A. A.; BOYLAN, J. E. On the variance of intermittent demand estimates. International Journal of Production Economics, v. 128, n. 2, p. 546-555, 2010.

SYNTETOS, A. A.; BOYLAN, J. E.; CROSTON, J. D. On the categorization of demand patterns. Journal of the Operational Research Society, v. 56, n. 5, p. 495-503, 2005.

TEUNTER, R. H.; DUNCAN, L. Forecasting intermittent demand: A comparative study. Journal of the Operational Research Society, v. 60, n. 3, p. 321-329, 2009.

TEUNTER, R. H.; SYNTETOS, A. A.; BABAI, M. Z. Intermittent demand: Linking forecasting to inventory obsolescence. European Journal of Operational Research, v. 214, n. 3, p. 606-615, 2011.

TEUNTER, R.; SANI, B. On the bias of Croston's forecasting method. European Journal of Operational Research, v. 194, n. 1, p. 177-183, 2009.

TUBINO, D. F. Manual de planejamento e controle da produção. 2. ed. São Paulo: Atlas, 2000.

WALLSTRÖM, P.; SEGERSTEDT, A. Evaluation of forecasting error measurements and techniques for intermittent demand. International Journal of Production Economics, v. 128 , n. 2, p. 625-636, 2010.

WILLEMAIN, T. R. et al. Forecasting intermittent demand in manufacturing: a comparative evaluation of Croston's method. International Journal of Forecasting, v. 10, n. 4, p. 529 538, 1994. 
APÊNDICE A - Classificação de erros de otimizações (demanda intermitente).

\begin{tabular}{|c|c|c|c|c|c|c|}
\hline \multirow{2}{*}{$\begin{array}{l}\text { DMAE } \\
\text { MMS3 }\end{array}$} & \multicolumn{6}{|c|}{ DESVIO MÉDIO ABSOLUTO ESCALONADO } \\
\hline & Treino & & & Teste & & \\
\hline Otimização & $\begin{array}{c}\text { a } 1 \text { passo } \\
32 \mathrm{p} .\end{array}$ & $\begin{array}{c}\text { Ranking } \\
\text { treino }\end{array}$ & $T+1$ & $T+3$ & $\mathrm{~T}+6$ & $\begin{array}{l}\text { Ranking } \\
\text { teste }\end{array}$ \\
\hline OT DMA & 1,560 & * & 1,691 & 1,706 & 1,703 & * \\
\hline OT DQM & 1,560 & * & 1,691 & 1,706 & 1,703 & * \\
\hline ОT TAM & 1,560 & * & 1,691 & 1,706 & 1,703 & * \\
\hline OT TQM & 1,560 & * & 1,691 & 1,706 & 1,703 & * \\
\hline NOT naïve & 1,560 & * & 1,691 & 1,706 & 1,703 & * \\
\hline NOT média & 1,560 & * & 1,691 & 1,706 & 1,703 & * \\
\hline SBA & Treino & & & Teste & & \\
\hline Otimização & $\begin{array}{c}\text { a } 1 \text { passo } \\
32 \mathrm{p} .\end{array}$ & $\begin{array}{c}\text { Ranking } \\
\text { treino }\end{array}$ & $\mathrm{T}+1$ & $T+3$ & $\mathrm{~T}+6$ & $\begin{array}{c}\text { Ranking } \\
\text { teste }\end{array}$ \\
\hline OT DMA & 1,000 & 1,00 & 1,127 & 1,144 & 1,108 & 1,00 \\
\hline OT DQM & 1,395 & 2,00 & 1,527 & 1,545 & 1,527 & 2,00 \\
\hline От TAM & 1,480 & 3,00 & 1,627 & 1,636 & 1,615 & 3,00 \\
\hline От TQM & 1,491 & 4,00 & 1,635 & 1,644 & 1,624 & 4,33 \\
\hline NOT naïve & 1,993 & 6,00 & 1,806 & 1,805 & 1,791 & 6,00 \\
\hline NOT média & 1,533 & 5,00 & 1,635 & 1,641 & 1,624 & 4,67 \\
\hline CROSTON & Treino & & & Teste & & \\
\hline Otimização & $\begin{array}{c}\text { a } 1 \text { passo } \\
32 \mathrm{p} .\end{array}$ & $\begin{array}{c}\text { Ranking } \\
\text { treino }\end{array}$ & $\mathrm{T}+1$ & $\mathrm{~T}+3$ & $\mathrm{~T}+6$ & $\begin{array}{c}\text { Ranking } \\
\text { teste }\end{array}$ \\
\hline OT DMA & 1,008 & 1,00 & 1,142 & 1,158 & 1,122 & 1,00 \\
\hline OT DQM & 1,405 & 2,00 & 1,569 & 1,587 & 1,571 & 2,00 \\
\hline OT TAM & 1,482 & 3,00 & 1,631 & 1,642 & 1,625 & 3,00 \\
\hline От TQM & 1,492 & 4,00 & 1,638 & 1,651 & 1,634 & 4,00 \\
\hline NOT naïve & 2,053 & 6,00 & 1,851 & 1,849 & 1,836 & 6,00 \\
\hline NOT média & 1,567 & 5,00 & 1,670 & 1,676 & 1,660 & 5,00 \\
\hline SBJ & Treino & & & Teste & & \\
\hline Otimização & $\begin{array}{c}\text { a } 1 \text { passo } \\
32 \mathrm{p} .\end{array}$ & $\begin{array}{c}\text { Ranking } \\
\text { treino }\end{array}$ & $\mathrm{T}+1$ & $\mathrm{~T}+3$ & $T+6$ & $\begin{array}{c}\text { Ranking } \\
\text { teste }\end{array}$ \\
\hline ОT DMA & 0,952 & 1,00 & 1,053 & 1,069 & 1,027 & 1,00 \\
\hline OT DQM & 1,387 & 2,00 & 1,510 & 1,527 & 1,507 & 2,00 \\
\hline ОТ TAM & 1,480 & 3,00 & 1,624 & 1,632 & 1,612 & 3,00 \\
\hline От TQM & 1,491 & 4,00 & 1,635 & 1,643 & 1,622 & 4,67 \\
\hline NOT naîve & 1,990 & 6,00 & 1,804 & 1,803 & 1,789 & 6,00 \\
\hline NOT média & 1,531 & 5,00 & 1,633 & 1,639 & 1,622 & 4,33 \\
\hline SES & Treino & & & Teste & & \\
\hline Otimização & $\begin{array}{c}\text { a } 1 \text { passo } \\
32 \mathrm{p} .\end{array}$ & $\begin{array}{c}\text { Ranking } \\
\text { treino }\end{array}$ & $T+1$ & $\mathrm{~T}+3$ & $\mathrm{~T}+6$ & $\begin{array}{c}\text { Ranking } \\
\text { teste }\end{array}$ \\
\hline OT DMA & 1,143 & 1,00 & 1,369 & 1,384 & 1,357 & 1,00 \\
\hline OT DQM & 1,473 & 2,00 & 1,625 & 1,637 & 1,622 & 6,00 \\
\hline ОТ ТАM & 1,509 & 4,00 & 1,592 & 1,600 & 1,580 & 2,00 \\
\hline От TQM & 1,526 & 6,00 & 1,598 & 1,606 & 1,586 & 3,67 \\
\hline NOT naïve & 1,495 & 3,00 & 1,593 & 1,606 & 1,589 & 4,00 \\
\hline NOT média & 1,521 & 5,00 & 1,592 & 1,608 & 1,591 & 4,33 \\
\hline ZERO & Treino & & & Teste & & \\
\hline Otimização & $\begin{array}{c}\text { a } 1 \text { passo } \\
32 \mathrm{p} .\end{array}$ & $\begin{array}{c}\text { Ranking } \\
\text { treino }\end{array}$ & $\mathrm{T}+1$ & $\mathrm{~T}+3$ & $\mathrm{~T}+6$ & $\begin{array}{c}\text { Ranking } \\
\text { teste }\end{array}$ \\
\hline ОT DMA & 1,000 & * & 1,018 & 1,039 & 0,992 & * \\
\hline OT DQM & 1,000 & * & 1,018 & 1,039 & 0,992 & * \\
\hline От TAM & 1,000 & * & 1,018 & 1,039 & 0,992 & * \\
\hline ОT TQM & 1,000 & * & 1,018 & 1,039 & 0,992 & * \\
\hline NOT naïve & 1,000 & * & 1,018 & 1,039 & 0,992 & * \\
\hline NOT média & 1,000 & * & 1,018 & 1,039 & 0,992 & * \\
\hline NAIVE & Treino & & & Teste & & \\
\hline Otimização & $\begin{array}{c}\text { a } 1 \text { passo } \\
32 \mathrm{p} .\end{array}$ & $\begin{array}{c}\text { Ranking } \\
\text { treino }\end{array}$ & $T_{+}$ & $\mathrm{T}+3$ & $T+6$ & $\begin{array}{c}\text { Ranking } \\
\text { teste }\end{array}$ \\
\hline OT DMA & 1,629 & * & 1,751 & 1,744 & 1,723 & * \\
\hline OT DQM & 1,629 & * & 1,751 & 1,744 & 1,723 & * \\
\hline ОT TAM & 1,629 & * & 1,751 & 1,744 & 1,723 & * \\
\hline OT TQM & 1,629 & * & 1,751 & 1,744 & 1,723 & * \\
\hline NOT naïve & 1,629 & * & 1,751 & 1,744 & 1,723 & * \\
\hline NOT média & 1,629 & * & 1,751 & 1,744 & 1,723 & * \\
\hline TSB & Treino & & & Teste & & \\
\hline Otimização & $\begin{array}{c}\text { a } 1 \text { passo } \\
32 \mathrm{p} .\end{array}$ & $\begin{array}{c}\text { Ranking } \\
\text { treino }\end{array}$ & $\mathrm{T}+1$ & $\mathrm{~T}+3$ & $\mathrm{~T}+6$ & $\begin{array}{c}\text { Ranking } \\
\text { teste }\end{array}$ \\
\hline OT DMA & 1,052 & 1,00 & 1,133 & 1,148 & 1,107 & 1,00 \\
\hline OT DQM & 1,474 & 2,00 & 1,616 & 1,627 & 1,607 & 6,00 \\
\hline ОТ TAM & 1,511 & 3,00 & 1,580 & 1,588 & 1,569 & 2,00 \\
\hline От TQM & 1,526 & 6,00 & 1,596 & 1,604 & 1,583 & 4,33 \\
\hline NOT naïve & 1,522 & 5,00 & 1,587 & 1,592 & 1,585 & 3,67 \\
\hline NOT média & 1,512 & 4,00 & 1,587 & 1,592 & 1,585 & 4,00 \\
\hline
\end{tabular}

\begin{tabular}{|c|c|c|c|c|c|c|}
\hline \multirow{3}{*}{$\begin{array}{l}\text { EMAE } \\
\text { MMS3 } \\
\text { Otimização }\end{array}$} & \multicolumn{6}{|c|}{ ERRO MÉDIO ABSOLUTO ESCALONADO } \\
\hline & Treino & & & Teste & & \\
\hline & $\begin{array}{c}\text { a } 1 \text { passo } \\
32 \mathrm{p} .\end{array}$ & $\begin{array}{c}\text { Ranking } \\
\text { treino }\end{array}$ & $T+1$ & $T+3$ & $T+6$ & $\begin{array}{c}\text { Ranking } \\
\text { teste }\end{array}$ \\
\hline OT DMA & 0,962 & * & 1,048 & 1,062 & 1,061 & * \\
\hline OT DQM & 0,962 & * & 1,048 & 1,062 & 1,061 & * \\
\hline OT TAM & 0,962 & * & 1,048 & 1,062 & 1,061 & * \\
\hline OT TQM & 0,962 & * & 1,048 & 1,062 & 1,061 & * \\
\hline NOT naïve & 0,962 & * & 1,048 & 1,062 & 1,061 & * \\
\hline NOT média & 0,962 & * & 1,048 & 1,062 & 1,061 & * \\
\hline SBA & Treino & & & Teste & & \\
\hline Otimização & $\begin{array}{c}\text { a } 1 \text { passo } \\
32 \mathrm{p} .\end{array}$ & $\begin{array}{l}\text { Ranking } \\
\text { treino }\end{array}$ & $T+1$ & $\mathrm{~T}+3$ & $\mathrm{~T}+6$ & $\begin{array}{c}\text { Ranking } \\
\text { teste }\end{array}$ \\
\hline OT DMA & 0,643 & 1,00 & 0,705 & 0,723 & 0,702 & 1,00 \\
\hline OT DQM & 0,874 & 2,00 & 0,951 & 0,967 & 0,959 & 2,00 \\
\hline OT TAM & 0,931 & 3,00 & 1,001 & 1,013 & 1,002 & 3,00 \\
\hline OT TQM & 0,936 & 4,00 & 1,006 & 1,018 & 1,007 & 5,00 \\
\hline NOT naïve & 1,238 & 6,00 & 1,099 & 1,105 & 1,097 & 6,00 \\
\hline NOT média & 0,963 & 5,00 & 1,004 & 1,014 & 1,005 & 4,00 \\
\hline CROSTON & Treino & & & Teste & & \\
\hline Otimização & $\begin{array}{c}\text { a } 1 \text { passo } \\
32 \mathrm{p} .\end{array}$ & $\begin{array}{l}\text { Ranking } \\
\text { treino }\end{array}$ & $T+1$ & $T+3$ & $T+6$ & $\begin{array}{c}\text { Ranking } \\
\text { teste }\end{array}$ \\
\hline OT DMA & 0,648 & 1,00 & 0,715 & 0,733 & 0,712 & 1,00 \\
\hline OT DQM & 0,880 & 2,00 & 0,980 & 0,997 & 0,990 & 2,00 \\
\hline OT TAM & 0,932 & 3,00 & 1,002 & 1,015 & 1,007 & 3,00 \\
\hline ОT TQM & 0,937 & 4,00 & 1,007 & 1,022 & 1,014 & 4,00 \\
\hline NOT naïve & 1,274 & 6,00 & 1,126 & 1,131 & 1,125 & 6,00 \\
\hline NOT média & 0,984 & 5,00 & 1,026 & 1,035 & 1,027 & 5,00 \\
\hline SBJ & Treino & & & Teste & & \\
\hline Otimização & $\begin{array}{c}\text { a } 1 \text { passo } \\
32 \mathrm{p} .\end{array}$ & $\begin{array}{l}\text { Ranking } \\
\text { treino }\end{array}$ & $T+1$ & $T+3$ & $\mathrm{~T}+6$ & $\begin{array}{c}\text { Ranking } \\
\text { teste }\end{array}$ \\
\hline OT DMA & 0,617 & 1,00 & 0,659 & 0,677 & 0,653 & 1,00 \\
\hline OT DQM & 0,870 & 2,00 & 0,943 & 0,959 & 0,949 & 2,00 \\
\hline OT TAM & 0,931 & 3,00 & 0,999 & 1,010 & 1,000 & 3,00 \\
\hline OT TQM & 0,936 & 4,00 & 1,006 & 1,016 & 1,006 & 5,00 \\
\hline NOT naïve & 1,236 & 6,00 & 1,098 & 1,103 & 1,096 & 6,00 \\
\hline NOT média & 0,962 & 5,00 & 1,003 & 1,013 & 1,004 & 4,00 \\
\hline SES & Treino & & & Teste & & \\
\hline Otimização & $\begin{array}{c}\text { a } 1 \text { passo } \\
32 \mathrm{p} .\end{array}$ & $\begin{array}{l}\text { Ranking } \\
\text { treino }\end{array}$ & $T+1$ & $T+3$ & $T+6$ & $\begin{array}{c}\text { Ranking } \\
\text { teste }\end{array}$ \\
\hline OT DMA & 0,726 & 1,00 & 0,890 & 0,906 & 0,892 & 1,00 \\
\hline OT DQM & 0,911 & 2,00 & 1,001 & 1,016 & 1,010 & 6,00 \\
\hline OT TAM & 0,942 & 4,00 & 0,979 & 0,989 & 0,979 & 2,67 \\
\hline OT TQM & 0,952 & 6,00 & 0,982 & 0,992 & 0,982 & 4,33 \\
\hline NOT naïve & 0,942 & 3,00 & 0,978 & 0,991 & 0,982 & 3,33 \\
\hline NOT média & 0,947 & 5,00 & 0,976 & 0,991 & 0,982 & 3,67 \\
\hline ZERO & Treino & & & Teste & & \\
\hline Otimização & $\begin{array}{c}\text { a } 1 \text { passo } \\
32 \mathrm{p} .\end{array}$ & $\begin{array}{l}\text { Ranking } \\
\text { treino }\end{array}$ & $T+1$ & $T+3$ & $T+6$ & $\begin{array}{c}\text { Ranking } \\
\text { teste }\end{array}$ \\
\hline OT DMA & 0,649 & * & 0,636 & 0,656 & 0,627 & * \\
\hline OT DQM & 0,649 & * & 0,636 & 0,656 & 0,627 & * \\
\hline OT TAM & 0,649 & * & 0,636 & 0,656 & 0,627 & * \\
\hline OT TQM & 0,649 & * & 0,636 & 0,656 & 0,627 & * \\
\hline NOT naïve & 0,649 & * & 0,636 & 0,656 & 0,627 & * \\
\hline NOT média & 0,649 & * & 0,636 & 0,656 & 0,627 & * \\
\hline NAVE & Treino & & & Teste & & \\
\hline Otimização & $\begin{array}{c}\text { a } 1 \text { passo } \\
32 \mathrm{p} .\end{array}$ & $\begin{array}{l}\text { Ranking } \\
\text { treino }\end{array}$ & $T+1$ & $\mathrm{~T}+3$ & $T+6$ & $\begin{array}{c}\text { Ranking } \\
\text { teste }\end{array}$ \\
\hline OT DMA & 1,000 & * & 1,142 & 1,142 & 1,131 & * \\
\hline OT DQM & 1,000 & * & 1,142 & 1,142 & 1,131 & * \\
\hline OT TAM & 1,000 & * & 1,142 & 1,142 & 1,131 & * \\
\hline OT TQM & 1,000 & * & 1,142 & 1,142 & 1,131 & * \\
\hline NOT naïve & 1,000 & * & 1,142 & 1,142 & 1,131 & * \\
\hline NOT média & 1,000 & * & 1,142 & 1,142 & 1,131 & * \\
\hline TSB & Treino & & & Teste & & \\
\hline Otimização & $\begin{array}{c}\text { a } 1 \text { passo } \\
32 \mathrm{p} .\end{array}$ & $\begin{array}{l}\text { Ranking } \\
\text { treino }\end{array}$ & II & 3 & 6 & $\begin{array}{c}\text { Ranking } \\
\text { teste }\end{array}$ \\
\hline OT DMA & 0,667 & 1,00 & 0,712 & 0,727 & 0,703 & 1,00 \\
\hline OT DQM & 0,911 & 2,00 & 1,002 & 1,015 & 1,005 & 6,00 \\
\hline OT TAM & 0,943 & 4,00 & 0,973 & 0,983 & 0,974 & 2,67 \\
\hline OT TQM & 0,951 & 6,00 & 0,982 & 0,992 & 0,982 & 5,00 \\
\hline NOT naïve & 0,951 & 5,00 & 0,976 & 0,983 & 0,980 & 3,67 \\
\hline NOT média & 0,939 & 3,00 & 0,976 & 0,983 & 0,980 & 2,67 \\
\hline
\end{tabular}


DQME DESVIO QUADRÁTICO MÉDIO ESCALONADO

\begin{tabular}{|c|c|c|c|c|c|c|}
\hline MMS3 & Treino & & & este & & \\
\hline Otimização & $\begin{array}{c}\text { a } 1 \text { passo } \\
32 \mathrm{p} .\end{array}$ & $\begin{array}{c}\text { Ranking } \\
\text { treino }\end{array}$ & $T+1$ & $T+3$ & $T+6$ & $\begin{array}{c}\text { Ranking } \\
\text { teste }\end{array}$ \\
\hline OT DMA & 9,175 & t & 25,088 & 20,735 & 20,077 & t \\
\hline OT DQM & 9,175 & * & 25,088 & 20,735 & 20,077 & * \\
\hline ОТ ТАМ & 9,175 & * & 25,088 & 20,735 & 20,077 & * \\
\hline ОT TQM & 9,175 & * & 25,088 & 20,735 & 20,077 & * \\
\hline NOT naïve & 9,175 & * & 25,088 & 20,735 & 20,077 & * \\
\hline NOT média & 9,175 & * & 25,088 & 20,735 & 20,077 & * \\
\hline SBA & Treino & & & te & & \\
\hline Otimização & $\begin{array}{c}\text { a } 1 \text { passo } \\
32 \mathrm{p} .\end{array}$ & $\begin{array}{c}\text { Ranking } \\
\text { treino }\end{array}$ & $T+1$ & $T+3$ & $\mathrm{~T}+6$ & $\begin{array}{c}\text { Ranking } \\
\text { teste }\end{array}$ \\
\hline OT DMA & 6,765 & 5,00 & 23,471 & 19,2 & 18 & 6,00 \\
\hline OT DQM & 5,957 & 1,00 & 22 & 18 & & 4,00 \\
\hline OT TAM & 6,385 & 3,00 & 22,681 & 18, & 17 & 1,67 \\
\hline OT TQM & 6,350 & 2,00 & 22,648 & 18,4 & 17 & 1,33 \\
\hline NOT naïve & 10,076 & 6,00 & 23, & 18 & & 5,00 \\
\hline NOT média & 6,597 & 4,00 & 22,751 & 18,482 & 17,678 & 3,00 \\
\hline CROSTON & Treino & & & Teste & & \\
\hline Otimização & $\begin{array}{c}\text { a } 1 \text { passo } \\
32 \mathrm{p} .\end{array}$ & $\begin{array}{c}\text { Ranking } \\
\text { treino }\end{array}$ & $T+1$ & $\mathrm{~T}+3$ & $\mathrm{~T}+6$ & $\begin{array}{c}\text { Ranking } \\
\text { teste }\end{array}$ \\
\hline OT DMA & 6,787 & 5,00 & 23,744 & 194 & 18 & 6,00 \\
\hline OT DQM & 5,982 & 1,00 & 23 & 4 & & 4,67 \\
\hline ОТ TAM & 6,393 & 3,00 & 22,595 & 18,432 & 17, & 1,00 \\
\hline ОT TQM & 6,355 & 2,00 & 22,6 & 18,4 & & 2,00 \\
\hline NOT naïve & 10,540 & 6,00 & 23 & 18 & & 4,33 \\
\hline NOT média & 6,646 & 4,00 & 22,7 & 18,4 & & 3,00 \\
\hline SBJ & Treino & & & & & \\
\hline Otimização & $\begin{array}{c}\text { a } 1 \text { passo } \\
32 \mathrm{p} .\end{array}$ & $\begin{array}{c}\text { Ranking } \\
\text { treino }\end{array}$ & $T+1$ & $T+3$ & $T+6$ & $\begin{array}{c}\text { Ranking } \\
\text { teste }\end{array}$ \\
\hline OT DMA & 6,877 & 5,00 & 23 , & 2 & 9 & 6,00 \\
\hline OT DQM & 5,960 & 1,00 & 22 & 18 & & 4,00 \\
\hline OT TAM & 6,387 & 3,00 & 22,6 & 18 & & 1,00 \\
\hline ОT TQM & 6,352 & 2,00 & 22 & 18 & & 2,00 \\
\hline NOT naïve & 10,053 & 6,00 & 23 & 18 & & 5,00 \\
\hline NOT média & 6,594 & 4,00 & 22,750 & 18,482 & 17,6 & 3,00 \\
\hline SES & Treino & & & & & \\
\hline O & $\begin{array}{c}\text { a } 1 \text { passo } \\
32 \mathrm{p} .\end{array}$ & $\begin{array}{c}\text { Ranking } \\
\text { treino }\end{array}$ & $T+1$ & $T+3$ & & $\begin{array}{r}\text { Ran } \\
\text { tes }\end{array}$ \\
\hline OT DMA & 8,524 & 6,00 & 26,786 & 2 & 2 & 6,00 \\
\hline OT DQM & 6,670 & 1,00 & 22, & 18,6 & & 5,00 \\
\hline ОТ TAM & 7,016 & 3,00 & 22,618 & 18,3 & 56 & 1,67 \\
\hline OT TQM & 7,003 & 2,00 & 22 , & 18 & & 2,33 \\
\hline NOT naïve & 7,968 & 5,00 & 22,623 & 18,4 & & 3,67 \\
\hline NOT média & 7,171 & 4,00 & 22,605 & 18,4 & 17 & 2,33 \\
\hline ERO & Treino & & & Teste & & \\
\hline Otimização & $\begin{array}{c}\text { a } 1 \text { passo } \\
32 \mathrm{p} .\end{array}$ & $\begin{array}{c}\text { Ranking } \\
\text { treino }\end{array}$ & $T+1$ & $T$ & $T_{+}$ & $\begin{array}{c}\text { Ranking } \\
\text { teste }\end{array}$ \\
\hline OT DMA & 7,689 & * & 23,6 & 10 & & * \\
\hline OT DQM & 7,689 & * & 23,6 & 1 & & * \\
\hline OT TAM & 7,689 & * & 23,662 & 19 & 18 & * \\
\hline OT TQM & 7,689 & * & 23,662 & 19,4 & & * \\
\hline NOT naïve & 7,689 & * & & & & * \\
\hline NOT média & 7,689 & * & 23 & 19,4 & & * \\
\hline NAIVE & Treino & & & Teste & & \\
\hline Otimização & $\begin{array}{c}\text { a } 1 \text { passo } \\
32 \mathrm{p} .\end{array}$ & $\begin{array}{c}\text { Ranking } \\
\text { treino }\end{array}$ & & & & $\begin{array}{c}\text { Ranking } \\
\text { teste }\end{array}$ \\
\hline OT DMA & 13,554 & * & & & & * \\
\hline OT DQM & 13,554 & * & 30,696 & 25 , & 25 & * \\
\hline OT TAM & 13,554 & * & 30,696 & 25,934 & & * \\
\hline OT TQM & 13,554 & * & 30,696 & 25 & & * \\
\hline NOT naïve & 13,554 & * & 30,696 & 25,934 & 25,042 & * \\
\hline NOT média & 13,554 & * & 30,696 & 25,934 & 25,042 & * \\
\hline TSB & Treino & & & Tede & & \\
\hline Otimização & $\begin{array}{c}\text { a } 1 \text { passo } \\
32 \mathrm{p} .\end{array}$ & $\begin{array}{c}\text { Ranking } \\
\text { treino }\end{array}$ & $\mathrm{T}$ & $T_{+}$ & $\mathrm{T}+$ & $\begin{array}{c}\text { Ranking } \\
\text { teste }\end{array}$ \\
\hline OT DMA & 7,565 & 4,00 & 23,7 & , & 68 & 6,00 \\
\hline OT DQM & 6,657 & 1,00 & 23, & 18,774 & 17 & 5,00 \\
\hline ОТ TAM & 7,034 & 3,00 & 22,5 & 18,360 & & 1,00 \\
\hline ОT TQM & 6,993 & 2,00 & 22,611 & 18,390 & 17,568 & 2,00 \\
\hline NOT naïve & 8,136 & 6,00 & 22,985 & 18,673 & 17,897 & 3,33 \\
\hline NOT média & 7,609 & 5,00 & 22,985 & 18,673 & 17,897 & 3,67 \\
\hline
\end{tabular}




\begin{tabular}{|c|c|c|c|c|c|c|}
\hline MMS3 & Treino & & & este & & \\
\hline Otimização & $\begin{array}{c}\text { a } 1 \text { passo } \\
32 \mathrm{p} .\end{array}$ & $\begin{array}{c}\text { Ranking } \\
\text { treino }\end{array}$ & $T+1$ & $T+3$ & $T+6$ & $\begin{array}{c}\text { Ranking } \\
\text { teste }\end{array}$ \\
\hline OT DMA & 27,562 & * & 1,189 & 3,562 & 7,119 & * \\
\hline OT DQM & 27,562 & * & 1,189 & 3,562 & 7,119 & * \\
\hline OT TAM & 27,562 & * & 1,189 & 3,562 & 7,119 & * \\
\hline OT TQM & 27,562 & * & 1,189 & 3,562 & 7,119 & * \\
\hline NOT naïve & 27,562 & * & 1,189 & 3,562 & 7,119 & * \\
\hline NOT média & 27,562 & * & 1,189 & 3,562 & 7,119 & * \\
\hline SBA & Treino & & & Teste & & \\
\hline Otimização & $\begin{array}{c}\text { a } 1 \text { passo } \\
32 \mathrm{p} .\end{array}$ & $\begin{array}{c}\text { Ranking } \\
\text { treino }\end{array}$ & $\mathrm{T}+1$ & $T+3$ & $T+6$ & $\begin{array}{c}\text { Ranking } \\
\text { teste }\end{array}$ \\
\hline OT DMA & 24,113 & 6,00 & 0,799 & 2 & , & 6,00 \\
\hline OT DQM & 12,993 & 4,00 & 0,409 & 1,251 & 2,558 & 4,00 \\
\hline OT TAM & 5,693 & 1,00 & 0,130 & 0,457 & 1,078 & 1,00 \\
\hline OT TQM & 5,700 & 2,00 & 0,135 & 0,474 & 1,108 & 2,00 \\
\hline NOT naïve & 22,294 & 5,00 & 0,433 & 1,325 & 2,731 & 5,00 \\
\hline NOT média & 10,022 & 3,00 & 0,244 & 0,763 & 1,616 & 3,00 \\
\hline CROSTON & Treino & & & te & & \\
\hline Otimização & $\begin{array}{c}\text { a } 1 \text { passo } \\
32 \mathrm{p} .\end{array}$ & $\begin{array}{c}\text { Ranking } \\
\text { treino }\end{array}$ & $T+1$ & $T+3$ & $T+6$ & $\begin{array}{c}\text { Ranking } \\
\text { teste }\end{array}$ \\
\hline OT DMA & 24,126 & 6,00 & 0,8 & 2,439 & 4,8 & 6,00 \\
\hline OT DQM & 12,725 & 4,00 & 0,397 & 1,216 & 2,495 & 4,00 \\
\hline OT TAM & 5,691 & 1,00 & 0,137 & 0,480 & 1,124 & 1,00 \\
\hline OT TQM & 5,714 & 2,00 & 0,143 & 0,497 & 1,157 & 2,00 \\
\hline NOT naïve & 23,824 & 5,00 & 0,463 & 1,416 & 2,917 & 5,00 \\
\hline NOT média & 10,270 & 3,00 & 0,259 & 0,807 & 1,710 & 3,00 \\
\hline
\end{tabular}

\begin{tabular}{lcccccc} 
SBJ & \multicolumn{2}{c}{ Treino } & \multicolumn{5}{c}{ Teste } \\
Otimização & $\begin{array}{c}\text { a } 1 \text { passo } \\
32 \mathrm{p} \text {. }\end{array}$ & $\begin{array}{c}\text { Ranking } \\
\text { treino }\end{array}$ & $\mathrm{T}+1$ & $\mathrm{~T}+3$ & $\mathrm{~T}+6$ & $\begin{array}{c}\text { Ranking } \\
\text { teste }\end{array}$ \\
OT DMA & 26,590 & 6,00 & 0,883 & 2,656 & 5,302 & 6,00 \\
OT DQM & 13,148 & 4,00 & 0,406 & 1,240 & 2,534 & 4,00 \\
OT TAM & 5,707 & 1,00 & 0,130 & 0,456 & 1,071 & 1,00 \\
OT TQM & 5,712 & 2,00 & 0,136 & 0,473 & 1,105 & 2,00 \\
NOT naïve & 22,217 & 5,00 & 0,431 & 1,321 & 2,723 & 5,00 \\
NOT média & 10,013 & 3,00 & 0,243 & 0,761 & 1,612 & 3,00
\end{tabular}

\begin{tabular}{lcccccc} 
SES & \multicolumn{2}{c}{ Treino } & \multicolumn{5}{c}{ Teste } \\
Otimização & $\begin{array}{c}\text { 1 passo } \\
32 \mathrm{p} .\end{array}$ & Ranking & $\mathrm{t}+1$ & $\mathrm{~T}+3$ & $\mathrm{~T}+6$ & $\begin{array}{c}\text { Ranking } \\
\text { teste }\end{array}$ \\
OT DMA & 29,587 & 6,00 & 1,123 & 3,373 & 6,738 & 6,00 \\
OT DQM & 14,331 & 5,00 & 0,192 & 0,657 & 1,496 & 3,00 \\
OT TAM & 7,980 & 1,00 & 0,104 & 0,360 & 0,865 & 2,00 \\
OT TQM & 8,371 & 2,00 & 0,098 & 0,349 & 0,850 & 1,00 \\
NOT naïve & 12,976 & 4,00 & 0,319 & 0,976 & 1,999 & 4,00 \\
NOT média & 11,820 & 3,00 & 0,319 & 0,977 & 2,002 & 5,00
\end{tabular}

\begin{tabular}{|c|c|c|c|c|c|c|}
\hline ZERO & Treino & & & Teste & & \\
\hline Otimização & $\begin{array}{c}\text { a } 1 \text { passo } \\
32 \mathrm{p} .\end{array}$ & $\begin{array}{c}\text { Ranking } \\
\text { treino }\end{array}$ & $\mathrm{T}+1$ & $T+3$ & $T+6$ & $\begin{array}{c}\text { Ranking } \\
\text { teste }\end{array}$ \\
\hline OT DMA & 31,993 & * & 1,001 & 3,008 & 6,006 & * \\
\hline OT DQM & 31,993 & * & 1,001 & 3,008 & 6,006 & * \\
\hline ОТ TAM & 31,993 & * & 1,001 & 3,008 & 6,006 & * \\
\hline OT TQM & 31,993 & * & 1,001 & 3,008 & 6,006 & * \\
\hline NOT naïve & 31,993 & * & 1,001 & 3,008 & 6,006 & * \\
\hline NOT média & 31,993 & * & 1,001 & 3,008 & 6,006 & * \\
\hline NAIVE & Treino & & & este & & \\
\hline Otimização & $\begin{array}{c}\text { a } 1 \text { passo } \\
32 \mathrm{p} .\end{array}$ & $\begin{array}{c}\text { Ranking } \\
\text { treino }\end{array}$ & $T+1$ & $\mathrm{~T}+3$ & $T+6$ & $\begin{array}{c}\text { Ranking } \\
\text { teste }\end{array}$ \\
\hline OT DMA & 43,222 & * & 1,575 & 4,724 & 9,434 & * \\
\hline OT DQM & 43,222 & * & 1,575 & 4,724 & 9,434 & * \\
\hline ОТ TAM & 43,222 & * & 1,575 & 4,724 & 9,434 & * \\
\hline OT TQM & 43,222 & * & 1,575 & 4,724 & 9,434 & * \\
\hline NOT naïve & 43,222 & * & 1,575 & 4,724 & 9,434 & * \\
\hline NOT média & 43,222 & * & 1,575 & 4,724 & 9,434 & * \\
\hline TSB & Treino & & & Teste & & \\
\hline Otimização & $\begin{array}{c}\text { a } 1 \text { passo } \\
32 \mathrm{p} .\end{array}$ & $\begin{array}{c}\text { Ranking } \\
\text { treino }\end{array}$ & $\mathrm{T}+1$ & $T+3$ & $T+6$ & $\begin{array}{c}\text { Ranking } \\
\text { teste }\end{array}$ \\
\hline OT DMA & 26,403 & 6,00 & 0,855 & 2,5 & & 6,00 \\
\hline OT DQM & 13,459 & 3,00 & 0,297 & 0,933 & 1,970 & 3,00 \\
\hline OT TAM & 6,522 & 1,00 & 0,097 & 0,340 & 0,824 & 1,67 \\
\hline OT TQM & 6,864 & 2,00 & 0,091 & 0,334 & 0,829 & 1,33 \\
\hline NOT naïve & 14,861 & 4,00 & 0,519 & 1,562 & 3,144 & 4,00 \\
\hline NOT média & 15,693 & 5,00 & 0,519 & 1,562 & 3,145 & 5,00 \\
\hline
\end{tabular}

TQME TAXA DO QUADRADO DA MÉDIA ESCALONADA

\begin{tabular}{|c|c|c|c|c|c|c|}
\hline MMS3 & Treino & & & Teste & & \\
\hline Otimização & $\begin{array}{c}\text { a } 1 \text { passo } 32 \\
\text { p. }\end{array}$ & $\begin{array}{c}\text { Ranking } \\
\text { treino }\end{array}$ & $T+1$ & $T+3$ & $T+6$ & $\begin{array}{c}\text { Ranking } \\
\text { teste }\end{array}$ \\
\hline OT DMA & 51,735 & * & 2,805 & 8,418 & 16,940 & * \\
\hline OT DQM & 51,735 & * & 2,805 & 8,418 & 16,940 & * \\
\hline ОТ ТАM & 51,735 & * & 2,805 & 8,418 & 16,940 & * \\
\hline От TQM & 51,735 & * & 2,805 & 8,418 & 16,940 & * \\
\hline NOT naïve & 51,735 & * & 2,805 & 8,418 & 16,940 & * \\
\hline NOT média & 51,735 & * & 2,805 & 8,418 & 16,940 & * \\
\hline SBA & Treino & & & Teste & & \\
\hline Otimização & $\begin{array}{c}\text { a } 1 \text { passo } 32 \\
\text { p. }\end{array}$ & $\begin{array}{c}\text { Ranking } \\
\text { treino }\end{array}$ & $T+1$ & $T+3$ & $T+6$ & $\begin{array}{c}\text { Ranking } \\
\text { teste }\end{array}$ \\
\hline OT DMA & 31,405 & 5,00 & 0,831 & 2,554 & 5,249 & 6,00 \\
\hline OT DQM & 13,906 & 4,00 & 0,596 & 1,836 & 3,814 & 5,00 \\
\hline OT TAM & 5,229 & 2,00 & 0,065 & 0,248 & 0,658 & 1,00 \\
\hline OT TQM & 5,038 & 1,00 & 0,067 & 0,254 & 0,671 & 2,00 \\
\hline NOT naïve & 82,971 & 6,00 & 0,539 & 1,661 & 3,489 & 4,00 \\
\hline NOT média & 9,187 & 3,00 & 0,125 & 0,421 & 0,995 & 3,00 \\
\hline CROSTON & Treino & & & Teste & & \\
\hline Otimização & $\begin{array}{c}\text { a } 1 \text { passo } 32 \\
\text { p. }\end{array}$ & $\begin{array}{c}\text { Ranking } \\
\text { treino }\end{array}$ & $T+1$ & $T+3$ & $T+6$ & $\begin{array}{c}\text { Ranking } \\
\text { teste }\end{array}$ \\
\hline OT DMA & 31,290 & 5,00 & 1,032 & 3,157 & 6,461 & 6,00 \\
\hline OT DQM & 13,643 & 4,00 & 0,823 & 2,504 & 5,145 & 5,00 \\
\hline ОТ TAM & 5,230 & 2,00 & 0,120 & 0,407 & 0,973 & 1,00 \\
\hline ОТ TQM & 5,059 & 1,00 & 0,150 & 0,498 & 1,158 & 3,00 \\
\hline NOT naïve & 94,273 & 6,00 & 0,622 & 1,909 & 3,988 & 4,00 \\
\hline NOT média & 9,511 & 3,00 & 0,143 & 0,475 & 1,103 & 2,00 \\
\hline
\end{tabular}

\begin{tabular}{lcccccc} 
SBJ & \multicolumn{2}{c}{ Treino } & & \multicolumn{3}{c}{ Teste } \\
Otimização & $\begin{array}{c}\text { 1 passo 32 } \\
\text { p. }\end{array}$ & $\begin{array}{c}\text { Ranking } \\
\text { treino }\end{array}$ & $\mathrm{T}+1$ & $\mathrm{~T}+3$ & $\mathrm{~T}+6$ & $\begin{array}{c}\text { Ranking } \\
\text { teste }\end{array}$ \\
OT DMA & 36,433 & 5,00 & 0,874 & 2,685 & 5,509 & 6,00 \\
OT DQM & 14,237 & 4,00 & 0,501 & 1,548 & 3,229 & 4,00 \\
OT TAM & 5,245 & 2,00 & 0,050 & 0,203 & 0,567 & 1,00 \\
OT TQM & 5,041 & 1,00 & 0,059 & 0,229 & 0,618 & 2,00 \\
NOT naïve & 82,402 & 6,00 & 0,535 & 1,648 & 3,464 & 5,00 \\
NOT média & 9,176 & 3,00 & 0,124 & 0,419 & 0,991 & 3,00
\end{tabular}

\begin{tabular}{lcccccc} 
SES & \multicolumn{2}{c}{ Treino } & \multicolumn{5}{c}{ Teste } \\
Otimização & $\begin{array}{c}\text { a } 1 \text { passo } 32 \\
\text { p. }\end{array}$ & $\begin{array}{c}\text { Ranking } \\
\text { treino }\end{array}$ & $\mathrm{T}+1$ & $\mathrm{~T}+3$ & $\mathrm{~T}+6$ & $\begin{array}{c}\text { Ranking } \\
\text { teste }\end{array}$ \\
OT DMA & 72,567 & 6,00 & 4,109 & 12,390 & 24,928 & 6,00 \\
OT DQM & 19,843 & 4,00 & 0,514 & 1,569 & 3,265 & 5,00 \\
OT TAM & 12,532 & 1,00 & 0,039 & 0,161 & 0,470 & 2,00 \\
OT TQM & 12,720 & 2,00 & 0,034 & 0,149 & 0,444 & 1,00 \\
NOT naïve & 21,527 & 5,00 & 0,180 & 0,577 & 1,285 & 3,00 \\
NOT média & 14,265 & 3,00 & 0,181 & 0,583 & 1,295 & 4,00
\end{tabular}

\begin{tabular}{|c|c|c|c|c|c|c|}
\hline ZERO & Treino & & & Teste & & \\
\hline Otimização & $\begin{array}{c}\text { a } 1 \text { passo } 32 \\
\text { p. }\end{array}$ & $\begin{array}{c}\text { Ranking } \\
\text { treino }\end{array}$ & $T+1$ & $T+3$ & $T+6$ & $\begin{array}{l}\text { Ranking } \\
\text { teste }\end{array}$ \\
\hline OT DMA & 51,459 & * & 1,022 & 3,130 & 6,393 & * \\
\hline OT DQM & 51,459 & * & 1,022 & 3,130 & 6,393 & * \\
\hline ОТ TAM & 51,459 & * & 1,022 & 3,130 & 6,393 & * \\
\hline OT TQM & 51,459 & * & 1,022 & 3,130 & 6,393 & * \\
\hline NOT naïve & 51,459 & * & 1,022 & 3,130 & 6,393 & * \\
\hline NOT média & 51,459 & * & 1,022 & 3,130 & 6,393 & * \\
\hline NAIVE & Treino & & & Teste & & \\
\hline Otimização & $\begin{array}{c}\text { a } 1 \text { passo } 32 \\
\text { p. }\end{array}$ & $\begin{array}{c}\text { Ranking } \\
\text { treino }\end{array}$ & $T+1$ & $T+3$ & $\mathrm{~T}+6$ & $\begin{array}{c}\text { Ranking } \\
\text { teste }\end{array}$ \\
\hline OT DMA & 191,139 & * & 7,986 & 23,946 & 47,935 & * \\
\hline OT DQM & 191,139 & * & 7,986 & 23,946 & 47,935 & * \\
\hline ОТ TAM & 191,139 & * & 7,986 & 23,946 & 47,935 & * \\
\hline OT TQM & 191,139 & * & 7,986 & 23,946 & 47,935 & * \\
\hline NOT naïve & 191,139 & * & 7,986 & 23,946 & 47,935 & * \\
\hline NOT média & 191,139 & * & 7,986 & 23,946 & 47,935 & * \\
\hline TSB & Treino & & & Teste & & \\
\hline Otimização & $\begin{array}{c}\text { a } 1 \text { passo } 32 \\
\text { p. }\end{array}$ & $\begin{array}{c}\text { Ranking } \\
\text { treino }\end{array}$ & T & $T+3$ & T & $\begin{array}{c}\text { Ranking } \\
\text { teste }\end{array}$ \\
\hline OT DMA & 37,968 & 5,00 & 1,193 & 3,642 & 7,407 & 6,00 \\
\hline OT DQM & 14,108 & 2,00 & 0,545 & 1,669 & 3,458 & 3,67 \\
\hline ОТ TAM & 50,085 & 6,00 & 0,040 & 0,165 & 0,479 & 2,00 \\
\hline OT TQM & 7,428 & 1,00 & 0,034 & 0,151 & 0,454 & 1,00 \\
\hline NOT naïve & 19,043 & 4,00 & 0,550 & 1,676 & 3,455 & 3,67 \\
\hline NOT média & 16,912 & 3,00 & 0,551 & 1,677 & 3,457 & 4,67 \\
\hline
\end{tabular}




\begin{tabular}{|c|c|c|c|c|c|c|}
\hline MS3 & Treino & \multicolumn{5}{|c|}{ Teste } \\
\hline Otimização & $\begin{array}{c}\text { a } 1 \text { passo } \\
32 \mathrm{p} .\end{array}$ & $\begin{array}{c}\text { Ranking } \\
\text { treino }\end{array}$ & $\mathrm{T}+1$ & $T+3$ & $\mathrm{~T}+6$ & $\begin{array}{c}\text { Rankin } \\
\text { teste }\end{array}$ \\
\hline OT DMA & $-2,898$ & * & 0,136 & 0,643 & 3,034 & \\
\hline OT DQM & $-2,898$ & * & 0,136 & & & \\
\hline ОТ TAM & $-2,898$ & * & 0,136 & 0,643 & & \\
\hline ОT TQM & $-2,898$ & * & 0,136 & 0,643 & 3,034 & \\
\hline NOT naïve & $-2,898$ & * & 0,136 & 0,643 & 3,034 & \\
\hline NOT média & $-2,898$ & * & 0,136 & 0,643 & 3, & \\
\hline BA & Treino & \multicolumn{5}{|c|}{ Teste } \\
\hline Otimização & $\begin{array}{c}\text { a } 1 \text { passo } \\
32 \mathrm{p} .\end{array}$ & $\begin{array}{c}\text { Ranking } \\
\text { treino }\end{array}$ & $\mathrm{T}+1$ & $T$ & & . \\
\hline OT DMA & $-277,918$ & 5,00 & $-0,736$ & -4 & -1 & 5,00 \\
\hline DT DQM & 20,865 & 1,00 & -0 & & & 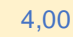 \\
\hline ОТ ТАM & 38,518 & 2,00 & 0,010 & -0 , & & 6 \\
\hline От TQM & 48,017 & 4,00 & 0,024 & -0 & & ,3 \\
\hline NOT naïve & 350,590 & 6,00 & 0,206 & 1,0 & & 50 \\
\hline 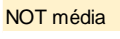 & & & & & & \\
\hline Ros & Treino & \multicolumn{5}{|c|}{ Teste } \\
\hline timiz & $\begin{array}{c}\text { a } 1 \text { passo } \\
32 \mathrm{p} .\end{array}$ & $\begin{array}{c}\text { Ranking } \\
\text { treino }\end{array}$ & $\mathrm{T}+1$ & - & & \\
\hline T DMA & $-277,906$ & 5,00 & $-0,722$ & $-4,508$ & -14 & 6,0 \\
\hline OT DQM & 21,653 & 1,00 & $-0,026$ & -0 , & & 2,0 \\
\hline ОТ TAM & 38,973 & 2,00 & 0,027 & -0 , & & 1,6 \\
\hline От TQM & 47,720 & 3,00 & 0,039 & 0, & 0 & 2,6 \\
\hline NOT naïve & 388,999 & 6,00 & 0,270 & 1, & & 50 \\
\hline NOT média & 62,493 & 4,00 & 0,073 & 0,265 & & 3 \\
\hline SBJ & Treino & \multicolumn{5}{|c|}{ Teste } \\
\hline Otimização & $\begin{array}{c}\text { a } 1 \text { passo } \\
32 \mathrm{p} .\end{array}$ & $\begin{array}{c}\text { Ranking } \\
\text { treino }\end{array}$ & +1 & $T+3$ & $\mathrm{~T}$ & tes \\
\hline DT DMA & $-317,455$ & 5,00 & $-0,857$ & $-5,316$ & -17 & 6,0 \\
\hline OT DQM & 17,114 & 1,00 & $-0,110$ & $-0,835$ & & 1 \\
\hline ОТ TAM & 37,906 & 2,00 & 0,006 & $-0,139$ & & 1,0 \\
\hline от TQM & 47,829 & 4,00 & 0,022 & $-0,044$ & 0,630 & 2,3 \\
\hline OT naïve & 348,569 & 6,00 & 0,202 & 1,038 & 4,417 & \\
\hline$T_{1}$ & & 300 & 010 & $-0,080$ & 0.504 & \\
\hline
\end{tabular}

\begin{tabular}{|c|c|c|c|c|c|c|}
\hline SES & Treino & & & Teste & & \\
\hline Otimização & $\begin{array}{c}\text { a } 1 \text { passo } \\
32 \mathrm{p} .\end{array}$ & $\begin{array}{c}\text { Ranking } \\
\text { treino }\end{array}$ & $T+1$ & $T+3$ & $\mathrm{~T}+6$ & $\begin{array}{c}\text { Ranking } \\
\text { teste }\end{array}$ \\
\hline OT DMA & $-250,679$ & 6,00 & $-0,414$ & $-2,659$ & $-8,522$ & 6,00 \\
\hline OT DQM & 3,307 & 2,00 & 0,039 & 0,060 & 0,993 & 3,67 \\
\hline OT TAM & 8,374 & 3,00 & $-0,028$ & $-0,342$ & $-0,413$ & 4,33 \\
\hline OT TQM & 24,011 & 5,00 & $-0,019$ & $-0,287$ & $-0,220$ & 3,33 \\
\hline NOT naïve & $-23,879$ & 4,00 & $-0,005$ & $-0,204$ & 0,072 & 2,00 \\
\hline NOT média & 1,565 & 1,00 & $-0,001$ & $-0,181$ & 0,150 & 1,67 \\
\hline ZERO & Treino & & & Teste & & \\
\hline Otimização & $\begin{array}{c}\text { a } 1 \text { passo } \\
32 \mathrm{p} .\end{array}$ & $\begin{array}{c}\text { Ranking } \\
\text { treino }\end{array}$ & $T+1$ & $T+3$ & $T+6$ & $\begin{array}{c}\text { Ranking } \\
\text { teste }\end{array}$ \\
\hline OT DMA & $-529,579$ & * & $-1,018$ & $-6,285$ & $-21,215$ & * \\
\hline OT DQM & $-529,579$ & * & $-1,018$ & $-6,285$ & $-21,215$ & * \\
\hline OT TAM & $-529,579$ & * & $-1,018$ & $-6,285$ & $-21,215$ & * \\
\hline OT TQM & $-529,579$ & * & $-1,018$ & $-6,285$ & $-21,215$ & * \\
\hline NOT naïve & $-529,579$ & * & $-1,018$ & $-6,285$ & $-21,215$ & * \\
\hline NOT média & $-529,579$ & * & $-1,018$ & $-6,285$ & $-21,215$ & * \\
\hline NAIVE & Treino & & & Teste & & \\
\hline Otimização & $\begin{array}{c}\text { a } 1 \text { passo } \\
32 \mathrm{p} .\end{array}$ & $\begin{array}{c}\text { Ranking } \\
\text { treino }\end{array}$ & $T+1$ & $T+3$ & $\mathrm{~T}+6$ & $\begin{array}{c}\text { Ranking } \\
\text { teste }\end{array}$ \\
\hline OT DMA & $-3,493$ & * & 0,082 & 0,318 & 1,896 & * \\
\hline OT DQM & $-3,493$ & * & 0,082 & 0,318 & 1,896 & * \\
\hline OT TAM & $-3,493$ & * & 0,082 & 0,318 & 1,896 & * \\
\hline OT TQM & $-3,493$ & * & 0,082 & 0,318 & 1,896 & * \\
\hline NOT naïve & $-3,493$ & * & 0,082 & 0,318 & 1,896 & * \\
\hline NOT média & $-3,493$ & * & 0,082 & 0,318 & 1,896 & * \\
\hline TSB & Treino & & & Teste & & \\
\hline Otimização & $\begin{array}{c}\text { a } 1 \text { passo } \\
32 \mathrm{p} .\end{array}$ & $\begin{array}{c}\text { Ranking } \\
\text { treino }\end{array}$ & $\mathrm{T}+1$ & $T+3$ & $T+6$ & $\begin{array}{c}\text { Ranking } \\
\text { teste }\end{array}$ \\
\hline OT DMA & $-381,101$ & 6,00 & $-0,745$ & $-4,643$ & $-15,467$ & 6,00 \\
\hline OT DQM & 7,039 & 2,00 & 0,028 & $-0,006$ & 0,764 & 3,33 \\
\hline OT TAM & 1,899 & 1,00 & $-0,043$ & $-0,435$ & $-0,737$ & 4,67 \\
\hline OT TQM & 17,061 & 3,00 & $-0,024$ & $-0,317$ & $-0,325$ & 3,33 \\
\hline NOT naïve & $-27,535$ & 5,00 & $-0,010$ & $-0,233$ & $-0,032$ & 2,33 \\
\hline NOT média & $-22,835$ & 4,00 & $-0,010$ & $-0,233$ & $-0,031$ & 1,33 \\
\hline
\end{tabular}

PEEEAm PERÍODO EM ESTOQUE ESCALONADO ABSOLUTO MODIFICADO

\begin{tabular}{|c|c|c|c|c|c|c|}
\hline MMS3 & Treino & & & Teste & & \\
\hline Otimização & $\begin{array}{c}\text { a } 1 \text { passo } \\
32 \mathrm{p} .\end{array}$ & $\begin{array}{c}\text { Ranking } \\
\text { treino }\end{array}$ & $\mathrm{T}+1$ & $\mathrm{~T}+3$ & $\mathrm{~T}+6$ & $\begin{array}{l}\text { Ranking } \\
\text { teste }\end{array}$ \\
\hline OT DMA & 67,449 & * & 1,691 & 8,886 & 28,956 & * \\
\hline OT DQM & 67,449 & * & 1,691 & 8,886 & 28,956 & * \\
\hline ОТ TAM & 67,449 & * & 1,691 & 8,886 & 28,956 & * \\
\hline ОT TQM & 67,449 & * & 1,691 & 8,886 & 28,956 & * \\
\hline NOT naïve & 67,449 & * & 1,691 & 8,886 & 28,956 & * \\
\hline NOT média & 67,449 & * & 1,691 & 8,886 & 28,956 & * \\
\hline
\end{tabular}

$\begin{array}{lccccccc}\text { SBA } & \begin{array}{c}\text { Treino } \\ \text { a 1 passo } \\ \text { Otimização }\end{array} & \begin{array}{c}\text { Ranking } \\ \text { 32 } \mathrm{p}\end{array} & \begin{array}{c}\text { treino } \\ \mathrm{T}+1\end{array} & \mathrm{~T}+3 & \mathrm{~T}+6 & \begin{array}{c}\text { Ranking } \\ \text { teste }\end{array} \\ \text { OT DMA } & 285,271 & 5,00 & 1,127 & 6,362 & 20,750 & 1,00 \\ \text { OT DQM } & 79,581 & 1,00 & 1,527 & 7,734 & 23,566 & 2,00 \\ \text { OT TAM } & 131,418 & 3,00 & 1,627 & 8,083 & 24,429 & 3,00 \\ \text { OT TQM } & 130,770 & 2,00 & 1,635 & 8,123 & 24,532 & 4,00 \\ \text { NOT naïv } & 499,495 & 6,00 & 1,806 & 9,040 & 27,692 & 6,00 \\ \text { NOT média } & 194,054 & 4,00 & 1,635 & 8,129 & 24,614 & 5,00\end{array}$

$\begin{array}{lccccccc}\text { CROSTON } & \begin{array}{c}\text { Treino } \\ \text { O1 passo } \\ \text { Otimização }\end{array} & \begin{array}{c}\text { Ranking } \\ \text { treino }\end{array} & \begin{array}{c}\text { T }+1 \\ \text { treste }\end{array} & \mathrm{T}+3 & \mathrm{~T}+6 & \begin{array}{c}\text { Ranking } \\ \text { teste }\end{array} \\ \text { OT DMA } & 292,589 & 5,00 & 1,142 & 6,486 & 21,150 & 1,00 \\ \text { OT DQM } & 81,303 & 1,00 & 1,569 & 7,904 & 24,220 & 2,00 \\ \text { OT TAM } & 131,739 & 3,00 & 1,631 & 8,095 & 24,510 & 3,00 \\ \text { OT TQM } & 130,578 & 2,00 & 1,638 & 8,143 & 24,680 & 4,00 \\ \text { NOT naïve } & 527,086 & 6,00 & 1,851 & 9,255 & 28,391 & 6,00 \\ \text { NOT média } & 199,952 & 4,00 & 1,670 & 8,286 & 25,115 & 5,00\end{array}$

$\begin{array}{lccccccc}\text { SBJ } & \begin{array}{c}\text { Treino } \\ \text { a 1 passo } \\ \text { Otimização }\end{array} & \begin{array}{c}\text { Ranking } \\ \text { treino }\end{array} & \begin{array}{c}\text { T+1 } \\ \text { Teste }\end{array} & \mathrm{T}+3 & \mathrm{~T}+6 & \begin{array}{c}\text { Ranking } \\ \text { teste }\end{array} \\ \text { OT DMA } & 318,778 & 5,00 & 1,053 & 6,134 & 20,296 & 1,00 \\ \text { OT DQM } & 75,338 & 1,00 & 1,510 & 7,617 & 23,140 & 2,00 \\ \text { OT TAM } & 131,681 & 3,00 & 1,624 & 8,059 & 24,350 & 3,00 \\ \text { OT TQM } & 130,398 & 2,00 & 1,635 & 8,119 & 24,519 & 4,33 \\ \text { NOT naïv } & 498,071 & 6,00 & 1,804 & 9,029 & 27,656 & 6,00 \\ \text { NOT média } & 193,782 & 4,00 & 1,633 & 8,121 & 24,589 & 4,67\end{array}$

\begin{tabular}{|c|c|c|c|c|c|c|}
\hline SES & Treino & & & este & & \\
\hline Otimização & $\begin{array}{c}\text { a } 1 \text { passo } \\
32 \mathrm{p} .\end{array}$ & $\begin{array}{c}\text { Ranking } \\
\text { treino }\end{array}$ & $T+1$ & $T+3$ & $T+6$ & $\begin{array}{c}\text { Ranking } \\
\text { teste }\end{array}$ \\
\hline OT DMA & 277,751 & 5,00 & 1,369 & 0, & & 2,67 \\
\hline OT DQM & 93,3 & 2,00 & 1,6 & 8,0 & & 5,67 \\
\hline OT TAM & 124, & 3.00 & 1,5 & 7,8 & 23 & 2,67 \\
\hline OT TQM & 127 & 4,00 & 1,5 & 7,909 & 23 & 4,67 \\
\hline NOT naïve & 320,406 & 6,00 & 1,5 & 7,889 & 23,634 & 3,33 \\
\hline JOT média & 28,567 & 1,00 & 1,592 & 7,881 & 23,624 & 2,00 \\
\hline
\end{tabular}

\begin{tabular}{|c|c|c|c|c|c|c|}
\hline ZERO & Treino & & & Teste & & \\
\hline Otimização & $\begin{array}{c}\text { a } 1 \text { passo } \\
32 \mathrm{p} .\end{array}$ & $\begin{array}{c}\text { Ranking } \\
\text { treino }\end{array}$ & $T+1$ & $T+3$ & $T+6$ & $\begin{array}{c}\text { Ranking } \\
\text { teste }\end{array}$ \\
\hline ОT DMA & 529,579 & * & 1,018 & 6,285 & 21,215 & 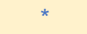 \\
\hline OT DQM & 529,579 & * & 1,018 & 6,285 & 21,215 & * \\
\hline ОТ TAM & 529,579 & * & 1,018 & 6,285 & 21,215 & * \\
\hline ОT TQM & 529,579 & * & 1,018 & 6,285 & 21,215 & * \\
\hline NOT naïve & 529,579 & * & 1,018 & 6,285 & 21,215 & * \\
\hline NOT média & 529,579 & * & 1,018 & 6,285 & 21,215 & * \\
\hline
\end{tabular}

\begin{tabular}{|c|c|c|c|c|c|c|}
\hline NAIVE & Treino & & & Teste & & \\
\hline Otimização & $\begin{array}{c}\text { a } 1 \text { passo } \\
32 \mathrm{p} .\end{array}$ & $\begin{array}{c}\text { Ranking } \\
\text { treino }\end{array}$ & $T+1$ & $T+3$ & $T+6$ & $\begin{array}{c}\text { Ranking } \\
\text { teste }\end{array}$ \\
\hline OT DMA & 44,607 & * & 1,751 & 9,953 & 33,625 & * \\
\hline OT DQM & 44,607 & * & 1,751 & 9,953 & 33,625 & * \\
\hline OT TAM & 44,607 & * & 1,751 & 9,953 & 33,625 & * \\
\hline OT TQM & 44,607 & * & 1,751 & 9,953 & 33,625 & * \\
\hline NOT naïve & 44,607 & * & 1,751 & 9,953 & 33,625 & * \\
\hline NOT média & 44,607 & * & 1,751 & 9,953 & 33,625 & * \\
\hline TSB & Treino & & & Teste & & \\
\hline Otimização & $\begin{array}{c}\text { a } 1 \text { passo } \\
32 \mathrm{p} .\end{array}$ & $\begin{array}{c}\text { Ranking } \\
\text { treino }\end{array}$ & $T+1$ & $T+3$ & $T+6$ & $\begin{array}{c}\text { Ranking } \\
\text { teste }\end{array}$ \\
\hline OT DMA & 391,301 & 6,00 & 1,133 & 6,542 & 21,449 & 1,00 \\
\hline OT DQM & 75,079 & 1,00 & 1,616 & 8,044 & 24,481 & 6,00 \\
\hline OT TAM & 123,991 & 2,00 & 1,580 & 7,839 & 23,586 & 2,00 \\
\hline OT TQM & 125,009 & 3,00 & 1,596 & 7,921 & 23,850 & 4,33 \\
\hline NOT naïve & 231,558 & 5,00 & 1,587 & 7,907 & 23,979 & 4,33 \\
\hline DT média & 15 & 00 & , & 907 & 年 & 333 \\
\hline
\end{tabular}




\section{APÊNDICE B - Classificação de erros dos modelos (demanda intermitente)}

\section{OTIMIZAÇÃO POR DMA}

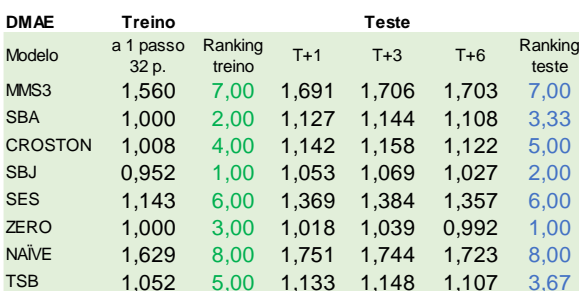

$\begin{array}{lcccccc}\text { DQME } & \begin{array}{c}\text { Treino } \\ \text { a 1 passo } \\ \text { Modelo }\end{array} & \begin{array}{c}\text { Ranking } \\ \text { treino }\end{array} & \begin{array}{c}\mathrm{T}+1 \\ \text { Teste }\end{array} & \mathrm{T}+3 & \mathrm{~T}+6 & \begin{array}{c}\text { Ranking } \\ \text { teste }\end{array} \\ \text { MMS3 } & 9,175 & 7,00 & 25,088 & 20,735 & 20,077 & 6,00 \\ \text { SBA } & 6,765 & 1,00 & 23,471 & 19,269 & 18,383 & 1,00 \\ \text { CROSTON } & 6,787 & 2,00 & 23,744 & 19,494 & 18,608 & 4,33 \\ \text { SBJ } & 6,877 & 3,00 & 23,529 & 19,322 & 18,419 & 2,00 \\ \text { SES } & 8,524 & 6,00 & 26,786 & 22,555 & 21,630 & 7,00 \\ \text { ZERO } & 7,689 & 5,00 & 23,662 & 19,471 & 18,557 & 3,00 \\ \text { NAIINE } & 13,554 & 8,00 & 30,696 & 25,934 & 25,042 & 8,00 \\ \text { TSB } & 7,565 & 4,00 & 23,734 & 19,594 & 18,668 & 4,67\end{array}$

\begin{tabular}{|c|c|c|c|c|c|c|}
\hline EMAE & Treino & \multicolumn{5}{|c|}{ Teste } \\
\hline Modelo & $\begin{array}{c}\text { a } 1 \text { passo } \\
32 \mathrm{p} .\end{array}$ & $\begin{array}{c}\text { Ranking } \\
\text { treino }\end{array}$ & $T+1$ & +3 & $T+6$ & $\begin{array}{c}\text { Ranking } \\
\text { teste }\end{array}$ \\
\hline MMS3 & 0,962 & 7,00 & 1,048 & 1,062 & 061 & 7,00 \\
\hline SBA & 0,643 & 2,00 & 0,705 & 0,723 & 0,702 & 3,00 \\
\hline CROSTON & 0,648 & 3,00 & 0,715 & 0,733 & 0,712 & 5,00 \\
\hline SBJ & 0,617 & 1,00 & 0,659 & 0,677 & 0,653 & 2,00 \\
\hline SES & 0,726 & 6,00 & 0,890 & 0,906 & 0,892 & 6,00 \\
\hline ZERO & 0,649 & 4,00 & 0,636 & 0,656 & 0,627 & 1,00 \\
\hline NAIIVE & 1,000 & 8,00 & 1,142 & 1,142 & 1,131 & 8,00 \\
\hline TSB & 0,667 & 5,00 & 0,712 & 0,727 & 0,703 & 4,00 \\
\hline PEEE & Treino & \multicolumn{5}{|c|}{ Teste } \\
\hline Modelo & $\begin{array}{c}\text { a } 1 \text { passo } \\
32 \mathrm{p} .\end{array}$ & $\begin{array}{c}\text { Ranking } \\
\text { treino }\end{array}$ & $T_{+}$ & +3 & $T+6$ & $\begin{array}{c}\text { Ranking } \\
\text { teste }\end{array}$ \\
\hline MMS3 & $-2,898$ & 1,00 & 0,136 & 0,643 & 3,034 & 2,00 \\
\hline SBA & $-277,918$ & 5,00 & $-0,736$ & $-4,595$ & $-15,297$ & 5,00 \\
\hline CROSTON & $-277,906$ & 4,00 & $-0,722$ & $-4,508$ & $-14,993$ & 4,00 \\
\hline SBJ & $-317,455$ & 6,00 & $-0,857$ & $-5,316$ & $-17,821$ & 7,00 \\
\hline ES & $-250,679$ & 3,00 & $-0,414$ & $-2,659$ & $-8,522$ & 3,00 \\
\hline ZERO & $-529,579$ & 8,00 & $-1,018$ & $-6,285$ & $-21,215$ & 8,00 \\
\hline NAIIIVE & $-3,493$ & 2,00 & 0,082 & 0,318 & 1,896 & 1,00 \\
\hline TSB & $-381,101$ & 7,00 & $-0,745$ & $-4,643$ & $-15,467$ & 6,00 \\
\hline
\end{tabular}

\begin{tabular}{|c|c|c|c|c|c|c|}
\hline AME & Treino & & & Teste & & \\
\hline Modelo & $\begin{array}{c}\text { a } 1 \text { passo } \\
32 \mathrm{p} .\end{array}$ & $\begin{array}{c}\text { Ranking } \\
\text { treino }\end{array}$ & $T+1$ & +3 & +6 & $\begin{array}{c}\text { Ranking } \\
\text { teste }\end{array}$ \\
\hline MMS3 & 27,562 & 5,00 & 1,189 & 3,562 & 7,119 & 7,00 \\
\hline BA & 24,113 & 1,00 & 0,799 & & & 1,00 \\
\hline ROSTON & 24,126 & 2,00 & 0,810 & 2,439 & 874 & 2,00 \\
\hline SBJ & 26,590 & 4,00 & 0,883 & 2,656 & 302 & 4,00 \\
\hline SES & 29,587 & 6,00 & 1,123 & 3,373 & ,738 & 6,00 \\
\hline ERO & 31,993 & 7,00 & 1,001 & 3,008 &, 006 & 5,00 \\
\hline AIIVE & 43,222 & 8,00 & 1,575 & 4,724 & ,434 & 8,00 \\
\hline TSB & 26,403 & 3,00 & 0,855 & 2,575 & 151 & 3,00 \\
\hline TQME & Treino & & & e & & \\
\hline Modelo & $\begin{array}{c}\text { a } 1 \text { passo } \\
32 \mathrm{p} .\end{array}$ & $\begin{array}{l}\text { Ranking } \\
\text { treino }\end{array}$ & & $T+3$ & & $\begin{array}{c}\text { Ranking } \\
\text { teste }\end{array}$ \\
\hline MS3 & 51,735 & 6,00 & 2,805 & 8,4 & 16 & 6,00 \\
\hline BA & 31,405 & 2,00 & 0,831 & 2 & & 1,00 \\
\hline CROSTON & 31,290 & 1,00 & 1,032 & 3,157 & 6,461 & 4,00 \\
\hline SBJ & 36,433 & 3,00 & 0,874 & 2,685 & 5,509 & 2,00 \\
\hline SES & 72,567 & 7,00 & 4,109 & 12,390 & 24,928 & 7,00 \\
\hline ZERO & 51,459 & 5,00 & 1,022 & 3,130 & 6,393 & 3,00 \\
\hline NAIIVE & 191,139 & 8,00 & 7,986 & 23,946 & 47,935 & 8,00 \\
\hline TSB & 37,968 & 4,00 & 1,193 & 3,642 & 7,407 & 5,00 \\
\hline PEEEA & T & & & Teste & & \\
\hline Modelo & $\begin{array}{c}\text { a } 1 \text { passo } \\
32 \mathrm{p} .\end{array}$ & $\begin{array}{c}\text { Ranking } \\
\text { treino }\end{array}$ & $T+1$ & $T+3$ & $T+6$ & $\begin{array}{c}\text { Ranking } \\
\text { teste }\end{array}$ \\
\hline MMS3 & 67,449 & 2,00 & 1,691 & 8,8 & 28, & 7,00 \\
\hline SBA & 285,271 & 4,00 & 1,127 & 6,362 & 20,750 & 2,67 \\
\hline CROSTON & 292,589 & 5,00 & 1,142 & 6,486 & 21,150 & 4,00 \\
\hline SBJ & 318,778 & 6,00 & 1,053 & 6,134 & 20,296 & 1,33 \\
\hline SES & 277,751 & 3,00 & 1,369 & 7,835 & 26,078 & 6,00 \\
\hline ZERO & 529,579 & 8,00 & 1,018 & 6,285 & 21,215 & 2,33 \\
\hline NAIIVE & 44,607 & 1,00 & 1,751 & 9,953 & 33,625 & 8,00 \\
\hline
\end{tabular}

\section{OTIMIZAÇÃO POR DQM}

\begin{tabular}{|c|c|c|c|c|c|c|}
\hline MAE & Treino & & & Teste & & \\
\hline Modelo & $\begin{array}{c}\text { a } 1 \text { passo } \\
32 \mathrm{p} .\end{array}$ & $\begin{array}{c}\text { Ranking } \\
\text { treino }\end{array}$ & $\mathrm{T}$ & $T+3$ & $T+6$ & $\begin{array}{c}\text { Ranking } \\
\text { teste }\end{array}$ \\
\hline MS3 & 1,560 & 7,00 & 1 & 1,706 & 3 & \\
\hline $3 A$ & 1, & 3,00 & & & & ,00 \\
\hline ROS & 1,405 & 00 & 1,569 & 1,587 & 1,571 & 00 \\
\hline 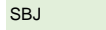 & $1,3 \varepsilon$ &, 00 & 1,510 & 1,5 & 1,5 &, 00 \\
\hline ES & 1,4 & 00 & 1,625 & 1,637 & 1,622 & 6,00 \\
\hline ERO & 1,000 & 1,00 & 1,018 & 1,039 & 0,992 & 1,00 \\
\hline QIIVE & & 8,0 & 1,751 & 1,744 & 1,723 & 8,00 \\
\hline 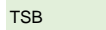 & 1,474 & 6,00 & 1,616 & 1,627 & 1,607 & 5,00 \\
\hline
\end{tabular}

$\begin{array}{lcccccc}\text { DQME } & \begin{array}{c}\text { Treino } \\ \text { M 1 passo } \\ \text { Modelo }\end{array} & \begin{array}{c}\text { Ranking } \\ \text { treino }\end{array} & \mathrm{T}+1 & \mathrm{~T}+3 & \mathrm{~T}+6 & \begin{array}{c}\text { Ranking } \\ \text { teste }\end{array} \\ \text { MMS3 } & 9,175 & 7,00 & 25,088 & 20,735 & 20,077 & 7,00 \\ \text { SBA } & 5,957 & 1,00 & 22,985 & 18,880 & 18,057 & 3,67 \\ \text { CROSTON } & 5,982 & 3,00 & 23,164 & 19,024 & 18,239 & 5,00 \\ \text { SBJ } & 5,960 & 2,00 & 22,949 & 18,789 & 17,941 & 2,67 \\ \text { SES } & 6,670 & 5,00 & 22,902 & 18,673 & 17,911 & 1,00 \\ \text { ZERO } & 7,689 & 6,00 & 23,662 & 19,471 & 18,557 & 6,00 \\ \text { NAIIVE } & 13,554 & 8,00 & 30,696 & 25,934 & 25,042 & 8,00 \\ \text { TSB } & 6,657 & 4,00 & 23,018 & 18,774 & 17,927 & 2,67\end{array}$

\begin{tabular}{|c|c|c|c|c|c|c|}
\hline IAE & in & & & Teste & & \\
\hline Modelo & $\begin{array}{c}\text { a } 1 \text { passo } \\
32 \mathrm{p} .\end{array}$ & $\begin{array}{c}\text { Ranking } \\
\text { treino }\end{array}$ & $T+1$ & $T+3$ & $T+6$ & $\begin{array}{c}\text { Ranking } \\
\text { teste }\end{array}$ \\
\hline AMS3 & 0,962 & 7,00 & 1,048 & $1,0 \epsilon$ & ,061 & 7,00 \\
\hline BA & & & & & & \\
\hline Ros & & & & & & \\
\hline 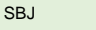 & & & & & & \\
\hline ES & & & 1, & & & 7 \\
\hline ERO & 649 & & 0,6 & 0, & 0,627 & 1,00 \\
\hline VAIIVE & 1,000 & 0 & 1,142 & 1,142 & 1,131 & 8,00 \\
\hline ISB & 0,911 & 5,00 & 1,002 & 1,015 & 1,005 & 5,33 \\
\hline
\end{tabular}

$\begin{array}{lcccccc}\text { PEEE } & \begin{array}{c}\text { Treino } \\ \text { M 1 passo } \\ \text { Modelo }\end{array} & \begin{array}{c}\text { Ranking } \\ \text { treino }\end{array} & \mathrm{T}+1 & \mathrm{~T}+3 & \mathrm{~T}+6 & \begin{array}{c}\text { Ranking } \\ \text { teste }\end{array} \\ \text { MMS3 } & -2,898 & 1,00 & 0,136 & 0,643 & 3,034 & 6,33 \\ \text { SBA } & 20,865 & 6,00 & -0,087 & -0,698 & -1,660 & 5,00 \\ \text { CROSTON } & 21,653 & 7,00 & -0,026 & -0,330 & -0,370 & 2,00 \\ \text { SBJ } & 17,114 & 5,00 & -0,110 & -0,835 & -2,140 & 6,33 \\ \text { SES } & 3,307 & 2,00 & 0,039 & 0,060 & 0,993 & 2,67 \\ \text { ZERO } & -529,579 & 8,00 & -1,018 & -6,285 & -21,215 & 8,00 \\ \text { NAIVE } & -3,493 & 3,00 & 0,082 & 0,318 & 1,896 & 4,00 \\ \text { TSB } & 7,039 & 4,00 & 0,028 & -0,006 & 0,764 & 1,67\end{array}$

$\begin{array}{lcccccc}\text { TAME } & \begin{array}{c}\text { Treino } \\ \text { a 1 passo } \\ \text { Modelo }\end{array} & \begin{array}{c}\text { Ranking } \\ \text { 32 } \mathrm{p} .\end{array} & \begin{array}{c}\mathrm{T}+1 \\ \text { treino }\end{array} & \begin{array}{c}\text { Teste } \\ \mathrm{T}+3\end{array} & \mathrm{~T}+6 & \begin{array}{c}\text { Ranking } \\ \text { teste }\end{array} \\ \text { MMS3 } & 27,562 & 6,00 & 1,189 & 3,562 & 7,119 & 7,00 \\ \text { SBA } & 12,993 & 2,00 & 0,409 & 1,251 & 2,558 & 5,00 \\ \text { CROSTON } & 12,725 & 1,00 & 0,397 & 1,216 & 2,495 & 3,00 \\ \text { SBJ } & 13,148 & 3,00 & 0,406 & 1,240 & 2,534 & 4,00 \\ \text { SES } & 14,331 & 5,00 & 0,192 & 0,657 & 1,496 & 1,00 \\ \text { ZERO } & 31,993 & 7,00 & 1,001 & 3,008 & 6,006 & 6,00 \\ \text { NAïV } & 43,222 & 8,00 & 1,575 & 4,724 & 9,434 & 8,00 \\ \text { TSB } & 13,459 & 4,00 & 0,297 & 0,933 & 1,970 & 2,00\end{array}$

\begin{tabular}{lcccccc} 
TQME & \multicolumn{1}{c}{ Treino } & \multicolumn{5}{c}{ Teste } \\
Modelo & $\begin{array}{c}\text { 1 passo } \\
32 \mathrm{p} .\end{array}$ & $\begin{array}{c}\text { Ranking } \\
\text { treino }\end{array}$ & $\mathrm{T}+1$ & $\mathrm{~T}+3$ & $\mathrm{~T}+6$ & $\begin{array}{c}\text { Ranking } \\
\text { teste }\end{array}$ \\
MMS3 & 51,735 & 7,00 & 2,805 & 8,418 & 16,940 & 7,00 \\
SBA & 13,906 & 2,00 & 0,596 & 1,836 & 3,814 & 4,00 \\
CROSTON & 13,643 & 1,00 & 0,823 & 2,504 & 5,145 & 5,00 \\
SBJ & 14,237 & 4,00 & 0,501 & 1,548 & 3,229 & 1,00 \\
SES & 19,843 & 5,00 & 0,514 & 1,569 & 3,265 & 2,00 \\
ZERO & 51,459 & 6,00 & 1,022 & 3,130 & 6,393 & 6,00 \\
NAIIVE & 191,139 & 8,00 & 7,986 & 23,946 & 47,935 & 8,00 \\
TSB & 14,108 & 3,00 & 0,545 & 1,669 & 3,458 & 3,00
\end{tabular}

\begin{tabular}{|c|c|c|c|c|c|c|}
\hline EEAm & Treino & & & este & & \\
\hline ode & $\begin{array}{c}\text { a } 1 \text { passo } \\
32 \mathrm{p} .\end{array}$ & $\begin{array}{c}\text { Ranking } \\
\text { treino }\end{array}$ & & & & $\begin{array}{c}\text { Ranking } \\
\text { teste }\end{array}$ \\
\hline MS3 & 67,449 & 2,00 & & & & \\
\hline 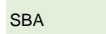 & & & & & & \\
\hline RO & 3 & 00 & 69 & 7,904 & & \\
\hline 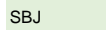 & 8 & 00 & 1,510 & 7,617 & 40 & 00 \\
\hline ES & & & 1,625 & 8,086 & 24, & 6,00 \\
\hline ERO & 29,579 & 8,00 & 1,018 & 6,285 & 21,215 & 1,00 \\
\hline AIIVE &, 607 & 1,00 & 1,751 & 9,953 & & 8,00 \\
\hline & 75,079 & 3,00 & 1,616 & 8,044 & 24,481 & 5,00 \\
\hline
\end{tabular}




\section{OTIMIZAÇÃO POR TAM}

\begin{tabular}{|c|c|c|c|c|c|c|}
\hline DMAE & Treino & & & Teste & & \\
\hline Modelo & $\begin{array}{c}\text { a } 1 \text { passo } \\
32 \mathrm{p} .\end{array}$ & $\begin{array}{c}\text { Ranking } \\
\text { treino }\end{array}$ & $T+1$ & $T+3$ & +6 & $\begin{array}{c}\text { Ranking } \\
\text { teste }\end{array}$ \\
\hline MMS3 & 1,560 & 7,00 & 1,691 & 1,706 & 1,703 & 7,00 \\
\hline SBA & 1,480 & 3,00 & 1,627 & 1,636 & 1,615 & 5,00 \\
\hline CROSTON & 1,482 & 4,00 & 1,631 & 1,642 & 1,625 & 6,00 \\
\hline SBJ & 1,480 & 2,00 & 1,624 & 1,632 & 1,612 & 4,00 \\
\hline SES & 1,509 & 5,00 & 1,592 & 1,600 & 1,580 & 3,00 \\
\hline ZERO & 1,000 & 1,00 & 1,018 & 1,039 & 0,992 & 1,00 \\
\hline NAIIVE & 1,629 & 8,00 & 1,751 & 1,744 & 1,723 & 8,00 \\
\hline TSB & 1,511 & 6,00 & 1,580 & 1,588 & 1,569 & 2,00 \\
\hline
\end{tabular}

\begin{tabular}{|c|c|c|c|c|c|c|}
\hline ME & ino & & & Teste & & \\
\hline Modelo & $\begin{array}{c}\text { a } 1 \text { passo } \\
32 \mathrm{p} .\end{array}$ & $\begin{array}{l}\text { Ranking } \\
\text { treino }\end{array}$ & $T+1$ & $T+3$ & $T+6$ & $\begin{array}{r}\text { Ran } \\
\text { tes }\end{array}$ \\
\hline 1S3 & 9,175 & 7,00 & 25,088 & 20,735 & 20,077 & \\
\hline A & 6 & 1,00 & & & & 4,67 \\
\hline os & 6 & 00 & 22 , & 18 & 17 & 3,67 \\
\hline & 6,387 & 00 & 22 , & 18, & 17 & ,33 \\
\hline & 6 & 0 & 22,618 & 18,367 & 17 &, $0 C$ \\
\hline & 7,689 & 0 & 23,662 & 19,471 & 18 &, 0 \\
\hline & 35 & 00 & 30,696 & 25,934 & 25,042 & 0 \\
\hline & & & & 8.360 & 5 & \\
\hline
\end{tabular}

\begin{tabular}{|c|c|c|c|c|c|c|}
\hline EMAE & reino & & & Teste & & \\
\hline Modelo & $\begin{array}{c}\text { a } 1 \text { passo } \\
32 \mathrm{p} .\end{array}$ & $\begin{array}{c}\text { Ranking } \\
\text { treino }\end{array}$ & $T+1$ & $T+3$ & $T+6$ & $\begin{array}{l}\text { Ranking } \\
\text { teste }\end{array}$ \\
\hline MMS3 & 0,962 & 7,00 & 1,048 & 1,062 & 1,061 & 7,00 \\
\hline SBA & 0,931 & 3,00 & 1,001 & 1,013 & 1,002 & 5,00 \\
\hline CROSTON & 0,932 & 4,00 & 1,002 & 1,015 & 1,007 & 6,00 \\
\hline SBJ & 0,931 & 2,00 & 0,999 & 1,010 & 1,000 & 4,00 \\
\hline SES & 0,942 & 5,00 & 0,979 & 0,989 & 0,979 & 3,00 \\
\hline ZERO & 0,649 & 1,00 & 0,636 & 0,656 & 0,627 & 1,00 \\
\hline NAIIVE & 1,000 & 8,00 & 1,142 & 1,142 & 1,131 & 8,00 \\
\hline SB & 943 & 00 & 0.973 & 0983 & 0.97 & 200 \\
\hline
\end{tabular}

\begin{tabular}{|c|c|c|c|c|c|c|}
\hline PEEE & Treino & & & Teste & & \\
\hline Modelo & $\begin{array}{c}\text { a } 1 \text { passo } \\
32 \mathrm{p} .\end{array}$ & $\begin{array}{c}\text { Ranking } \\
\text { treino }\end{array}$ & $T+1$ & $T+3$ & $T+6$ & $\begin{array}{l}\text { Ranking } \\
\text { teste }\end{array}$ \\
\hline MMS3 & $-2,898$ & 2,00 & 0,136 & 0,643 & 3,034 & 7,00 \\
\hline SBA & 38,518 & 6,00 & 0,010 & $-0,115$ & 0,383 & 2,00 \\
\hline CROSTON & 38,973 & 7,00 & 0,027 & $-0,012$ & 0,741 & 3,00 \\
\hline SBJ & 37,906 & 5,00 & 0,006 & $-0,139$ & 0,297 & 1,67 \\
\hline SES & 8,374 & 4,00 & $-0,028$ & $-0,342$ & $-0,413$ & 4,00 \\
\hline ZERO & $-529,579$ & 8,00 & $-1,018$ & $-6,285$ & $-21,215$ & 8,00 \\
\hline NAIIVE & $-3,493$ & 3,00 & 0,082 & 0,318 & 1,896 & 5,33 \\
\hline TSB & 1,899 & 1,00 & $-0,043$ & $-0,435$ & $-0,737$ & 5,00 \\
\hline TAME & Treino & & & Teste & & \\
\hline Modelo & $\begin{array}{c}\text { a } 1 \text { passo } \\
32 \mathrm{p} .\end{array}$ & $\begin{array}{c}\text { Ranking } \\
\text { treino }\end{array}$ & $T+1$ & & $T+6$ & $\begin{array}{c}\text { Ranking } \\
\text { teste }\end{array}$ \\
\hline MMS3 & 27,562 & 6,00 & 1,189 & 3,562 & 7,119 & 7,00 \\
\hline SBA & 5,693 & 2,00 & 0,130 & 0,457 & 1,078 & 4,00 \\
\hline CROSTON & 5,691 & 1,00 & 0,137 & 0,480 & 1,124 & 5,00 \\
\hline SBJ & 5,707 & 3,00 & 0,130 & 0,456 & 1,071 & 3,00 \\
\hline SES & 7,980 & 5,00 & 0,104 & 0,360 & 0,865 & 2,00 \\
\hline ZERO & 31,993 & 7,00 & 1,001 & 3,008 & 6,006 & 6,00 \\
\hline NAIIVE & 43,222 & 8,00 & 1,575 & 4,724 & 9,434 & 8,00 \\
\hline TSB & 6,522 & 4,00 & 0,097 & 0,340 & 0,824 & 1,00 \\
\hline
\end{tabular}

\begin{tabular}{|c|c|c|c|c|c|c|}
\hline TQME & Treino & & & Teste & & \\
\hline Modelo & $\begin{array}{c}\text { a } 1 \text { passo } \\
32 \mathrm{p} .\end{array}$ & $\begin{array}{c}\text { Ranking } \\
\text { treino }\end{array}$ & $\mathrm{T}+1$ & $T+3$ & $T+6$ & $\begin{array}{c}\text { Ranking } \\
\text { teste }\end{array}$ \\
\hline MMS3 & 51,735 & 7,00 & 2,805 & 8,418 & 16,940 & 7,00 \\
\hline SBA & 5,229 & 1,00 & 0,065 & 0,248 & 0,658 & 4,00 \\
\hline CROSTON & 5,230 & 2,00 & 0,120 & 0,407 & 0,973 & 5,00 \\
\hline SBJ & 5,245 & 3,00 & 0,050 & 0,203 & 0,567 & 3,00 \\
\hline SES & 12,532 & 5,00 & 0,039 & 0,161 & 0,470 & 1,00 \\
\hline ZERO & 51,459 & 6,00 & 1,022 & 3,130 & 6,393 & 6,00 \\
\hline NAIIVE & 191,139 & 8,00 & 7,986 & 23,946 & 47,935 & 8,00 \\
\hline TSB & 7,219 & 4,00 & 0,040 & 0,165 & 0,479 & 2,00 \\
\hline
\end{tabular}

$\begin{array}{lcccccc}\text { PEEEAm } & \begin{array}{c}\text { Treino } \\ \text { a 1 passo } \\ \text { Modelo }\end{array} & \begin{array}{c}\text { Ranking } \\ \text { treino }\end{array} & \begin{array}{c}\mathrm{T}+1 \\ \text { Meste }\end{array} & \mathrm{T}+3 & \mathrm{~T}+6 & \begin{array}{c}\text { Ranking } \\ \text { teste }\end{array} \\ \text { MMS3 } & 67,449 & 2,00 & 1,691 & 8,886 & 28,956 & 7,00 \\ \text { SBA } & 131,418 & 5,00 & 1,627 & 8,083 & 24,429 & 5,00 \\ \text { CROSTON } & 131,739 & 7,00 & 1,631 & 8,095 & 24,510 & 6,00 \\ \text { SBJ } & 131,681 & 6,00 & 1,624 & 8,059 & 24,350 & 4,00 \\ \text { SES } & 124,329 & 4,00 & 1,592 & 7,883 & 23,746 & 3,00 \\ \text { ZERO } & 529,579 & 8,00 & 1,018 & 6,285 & 21,215 & 1,00 \\ \text { NAIIVE } & 44,607 & 1,00 & 1,751 & 9,953 & 33,625 & 8,00 \\ \text { TSB } & 123,991 & 3,00 & 1,580 & 7,839 & 23,586 & 2,00\end{array}$

\section{OTIMIZAÇÃO POR TQM}

$\begin{array}{lcccccc}\text { DMAE } & \begin{array}{c}\text { Treino } \\ \text { a 1 passo } \\ \text { Modelo }\end{array} & \begin{array}{c}\text { Ranking } \\ \text { treino }\end{array} & \mathrm{T}+1 & \mathrm{~T}+3 & \mathrm{~T}+6 & \begin{array}{c}\text { Ranking } \\ \text { teste }\end{array} \\ \text { MMS3 } & 1,560 & 7,00 & 1,691 & 1,706 & 1,703 & 7,33 \\ \text { SBA } & 1,491 & 3,00 & 1,635 & 1,644 & 1,624 & 5,00 \\ \text { CROSTON } & 1,492 & 4,00 & 1,638 & 1,651 & 1,634 & 6,33 \\ \text { SBJ } & 1,491 & 2,00 & 1,635 & 1,643 & 1,622 & 4,67 \\ \text { SES } & 1,526 & 6,00 & 1,598 & 1,606 & 1,586 & 3,33 \\ \text { ZERO } & 1,000 & 1,00 & 1,018 & 0,996 & 0,992 & 1,00 \\ \text { NAIIVE } & 1,629 & 8,00 & 1,751 & 1,577 & 1,723 & 6,00 \\ \text { TSB } & 1,526 & 5,00 & 1,596 & 1,604 & 1,583 & 2,33\end{array}$

\begin{tabular}{lcccccc} 
DQME & \multicolumn{2}{c}{ Treino } & \multicolumn{5}{c}{ Teste } \\
Modelo & $\begin{array}{c}\text { a 1 passo } \\
32 \mathrm{p} .\end{array}$ & $\begin{array}{c}\text { Ranking } \\
\text { treino }\end{array}$ & $\mathrm{T}+1$ & $\mathrm{~T}+3$ & $\mathrm{~T}+6$ & $\begin{array}{c}\text { Ranking } \\
\text { teste }\end{array}$ \\
MMS3 & 9,175 & 7,00 & 25,088 & 20,735 & 20,077 & 7,00 \\
SBA & 6,350 & 1,00 & 22,648 & 18,452 & 17,643 & 4,33 \\
CROSTON & 6,355 & 3,00 & 22,603 & 18,451 & 17,690 & 3,33 \\
SBJ & 6,352 & 2,00 & 22,659 & 18,446 & 17,627 & 3,67 \\
SES & 7,003 & 5,00 & 22,623 & 18,374 & 17,551 & 1,67 \\
ZERO & 7,689 & 6,00 & 23,662 & 19,471 & 18,557 & 6,00 \\
NAIIVE & 13,554 & 8,00 & 30,696 & 25,934 & 25,042 & 8,00 \\
TSB & 6,993 & 4,00 & 22,611 & 18,390 & 17,568 & 2,00
\end{tabular}

\begin{tabular}{lcccccc} 
EMAE & Treino & \multicolumn{5}{c}{ Teste } \\
Modelo & $\begin{array}{c}\text { a 1 passo } \\
32 \mathrm{p} .\end{array}$ & $\begin{array}{c}\text { Ranking } \\
\text { treino }\end{array}$ & $\mathrm{T}+1$ & $\mathrm{~T}+3$ & $\mathrm{~T}+6$ & $\begin{array}{c}\text { Ranking } \\
\text { teste }\end{array}$ \\
MMS3 & 0,962 & 7,00 & 1,048 & 1,062 & 1,061 & 7,00 \\
SBA & 0,936 & 3,00 & 1,006 & 1,018 & 1,007 & 5,00 \\
CROSTON & 0,937 & 4,00 & 1,007 & 1,022 & 1,014 & 6,00 \\
SBJ & 0,936 & 2,00 & 1,006 & 1,016 & 1,006 & 4,00 \\
SES & 0,952 & 6,00 & 0,982 & 0,992 & 0,982 & 2,67 \\
ZERO & 0,649 & 1,00 & 0,636 & 0,711 & 0,627 & 1,00 \\
NAIIVE & 1,000 & 8,00 & 1,142 & 1,095 & 1,131 & 8,00 \\
TSB & 0,951 & 5,00 & 0,982 & 0,992 & 0,982 & 2,33
\end{tabular}

\begin{tabular}{lcccccc} 
PEEE & \multicolumn{2}{c}{ Treino } & \multicolumn{5}{c}{ Teste } \\
Modelo & $\begin{array}{c}\text { a 1 passo } \\
\text { Ranking }\end{array}$ & $\begin{array}{c}\text { Ran } \\
\text { treino }\end{array}$ & $\mathrm{T}+1$ & $\mathrm{~T}+3$ & $\mathrm{~T}+6$ & $\begin{array}{c}\text { Ranking } \\
\text { teste }\end{array}$ \\
MMS3 & $-2,898$ & 1,00 & 0,136 & 0,643 & 3,034 & 7,00 \\
SBA & 48,017 & 7,00 & 0,024 & $-0,031$ & 0,675 & 3,00 \\
CROSTON & 47,720 & 5,00 & 0,039 & 0,060 & 0,996 & 4,33 \\
SBJ & 47,829 & 6,00 & 0,022 & $-0,044$ & 0,630 & 2,33 \\
SES & 24,011 & 4,00 & $-0,019$ & $-0,287$ & $-0,220$ & 2,00 \\
ZERO & $-529,579$ & 8,00 & $-1,018$ & $-6,285$ & $-21,215$ & 8,00 \\
NAIVE & $-3,493$ & 2,00 & 0,082 & 0,318 & 1,896 & 6,00 \\
TSB & 17,061 & 3,00 & $-0,024$ & $-0,317$ & $-0,325$ & 3,33
\end{tabular}

\begin{tabular}{lcccccc} 
TAME & \multicolumn{2}{c}{ Treino } & \multicolumn{5}{c}{ Teste } \\
Modelo & $\begin{array}{c}\text { a 1 passo } \\
\text { 32 p. }\end{array}$ & $\begin{array}{c}\text { Ranking } \\
\text { treino }\end{array}$ & $\mathrm{T}+1$ & $\mathrm{~T}+3$ & $\mathrm{~T}+6$ & $\begin{array}{c}\text { Ranking } \\
\text { teste }\end{array}$ \\
MMS3 & 27,562 & 6,00 & 1,189 & 3,562 & 7,119 & 7,00 \\
SBA & 5,700 & 1,00 & 0,135 & 0,474 & 1,108 & 3,67 \\
CROSTON & 5,714 & 3,00 & 0,143 & 0,497 & 1,157 & 5,00 \\
SBJ & 5,712 & 2,00 & 0,136 & 0,473 & 1,105 & 3,33 \\
SES & 8,371 & 5,00 & 0,098 & 0,349 & 0,850 & 2,00 \\
ZERO & 31,993 & 7,00 & 1,001 & 3,008 & 6,006 & 6,00 \\
NAIIVE & 43,222 & 8,00 & 1,575 & 4,724 & 9,434 & 8,00 \\
TSB & 6,864 & 4,00 & 0,091 & 0,334 & 0,829 & 1,00
\end{tabular}

$\begin{array}{lcccccc}\text { TQME } & \begin{array}{c}\text { Treino } \\ \text { a 1 passo } \\ \text { Modelo }\end{array} & \begin{array}{c}\text { Ranking } \\ \text { treino }\end{array} & \begin{array}{c}\text { T+1 } \\ \text { Meste }\end{array} & \mathrm{T}+3 & \mathrm{~T}+6 & \begin{array}{c}\text { Ranking } \\ \text { teste }\end{array} \\ \text { MMS3 } & 51,735 & 7,00 & 2,805 & 8,418 & 16,940 & 7,00 \\ \text { SBA } & 5,038 & 1,00 & 0,067 & 0,254 & 0,671 & 4,00 \\ \text { CROSTON } & 5,059 & 3,00 & 0,150 & 0,498 & 1,158 & 5,00 \\ \text { SBJ } & 5,041 & 2,00 & 0,059 & 0,229 & 0,618 & 3,00 \\ \text { SES } & 12,720 & 5,00 & 0,034 & 0,149 & 0,444 & 1,33 \\ \text { ZERO } & 51,459 & 6,00 & 1,022 & 3,130 & 6,393 & 6,00 \\ \text { NAIIVE } & 191,139 & 8,00 & 7,986 & 23,946 & 47,935 & 8,00 \\ \text { TSB } & 7,428 & 4,00 & 0,034 & 0,151 & 0,454 & 1,67\end{array}$

\begin{tabular}{lcccccc} 
PEEEAm & \multicolumn{1}{c}{ Treino } & \multicolumn{5}{c}{ Teste } \\
Modelo & $\begin{array}{c}\text { a 1 passo } \\
32 \mathrm{p} .\end{array}$ & $\begin{array}{c}\text { Ranking } \\
\text { treino }\end{array}$ & $\mathrm{T}+1$ & $\mathrm{~T}+3$ & $\mathrm{~T}+6$ & $\begin{array}{c}\text { Ranking } \\
\text { teste }\end{array}$ \\
MMS3 & 67,449 & 2,00 & 1,691 & 8,886 & 28,956 & 7,00 \\
SBA & 130,770 & 7,00 & 1,635 & 8,123 & 24,532 & 4,67 \\
CROSTON & 130,578 & 6,00 & 1,638 & 8,143 & 24,680 & 6,00 \\
SBJ & 130,398 & 5,00 & 1,635 & 8,119 & 24,519 & 4,33 \\
SES & 127,562 & 4,00 & 1,598 & 7,909 & 23,808 & 2,33 \\
ZERO & 529,579 & 8,00 & 1,018 & 6,021 & 21,215 & 1,00 \\
NAIIVE & 44,607 & 1,00 & 1,751 & 8,938 & 33,625 & 8,00 \\
TSB & 125,009 & 3,00 & 1,596 & 7,921 & 23,850 & 2,67
\end{tabular}




\section{NÃO OTIMIZADO (INÍCIO Pt = NAIVE)}

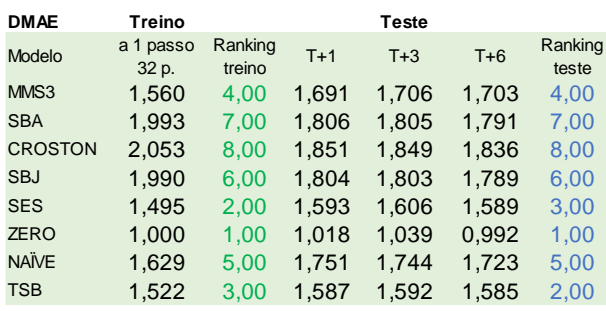

$\begin{array}{lcccccc}\text { DQME } & \begin{array}{c}\text { Treino } \\ \text { a 1 passo } \\ \text { Modelo }\end{array} & \begin{array}{c}\text { Ranking } \\ \text { treino }\end{array} & \mathrm{T}+1 & \mathrm{~T}+3 & \mathrm{~T}+6 & \begin{array}{c}\text { Ranking } \\ \text { teste }\end{array} \\ \text { MMS3 } & 9,175 & 4,00 & 25,088 & 20,735 & 20,077 & 7,00 \\ \text { SBA } & 10,076 & 6,00 & 23,258 & 18,915 & 18,145 & 4,00 \\ \text { CROSTON } & 10,540 & 7,00 & 23,345 & 18,995 & 18,232 & 5,00 \\ \text { SBJ } & 10,053 & 5,00 & 23,254 & 18,912 & 18,140 & 3,00 \\ \text { SES } & 7,968 & 2,00 & 22,623 & 18,411 & 17,606 & 1,00 \\ \text { ZERO } & 7,689 & 1,00 & 23,662 & 19,471 & 18,557 & 6,00 \\ \text { NAïV } & 13,554 & 8,00 & 30,696 & 25,934 & 25,042 & 8,00 \\ \text { TSB } & 8,136 & 3,00 & 22,985 & 18,673 & 17,897 & 2,00\end{array}$

$\begin{array}{lcccccc}\text { EMAE } & \begin{array}{c}\text { Treino } \\ \text { a 1 passo } \\ \text { Modelo } \mathrm{p}\end{array} & \begin{array}{c}\text { Ranking } \\ \text { treino }\end{array} & \mathrm{T}+1 & \mathrm{~T}+3 & \mathrm{~T}+6 & \begin{array}{c}\text { Ranking } \\ \text { teste }\end{array} \\ \text { MMS3 } & 0,962 & 4,00 & 1,048 & 1,062 & 1,061 & 4,00 \\ \text { SBA } & 1,238 & 7,00 & 1,099 & 1,105 & 1,097 & 6,00 \\ \text { CROSTON } & 1,274 & 8,00 & 1,126 & 1,131 & 1,125 & 7,00 \\ \text { SBJ } & 1,236 & 6,00 & 1,098 & 1,103 & 1,096 & 5,00 \\ \text { SES } & 0,942 & 2,00 & 0,978 & 0,991 & 0,982 & 3,00 \\ \text { ZERO } & 0,649 & 1,00 & 0,636 & 0,656 & 0,627 & 1,00 \\ \text { NAïV } & 1,000 & 5,00 & 1,142 & 1,142 & 1,131 & 8,00 \\ \text { TSB } & 0,951 & 3,00 & 0,976 & 0,983 & 0,980 & 2,00\end{array}$

\begin{tabular}{lcccccc} 
PEEE & Treino & \multicolumn{5}{c}{ Teste } \\
Modelo & $\begin{array}{c}\text { a 1 passo } \\
32 \mathrm{p} .\end{array}$ & $\begin{array}{c}\text { Ranking } \\
\text { treino }\end{array}$ & $\mathrm{T}+1$ & $\mathrm{~T}+3$ & $\mathrm{~T}+6$ & $\begin{array}{c}\text { Ranking } \\
\text { teste }\end{array}$ \\
MMS3 & $-2,898$ & 1,00 & 0,136 & 0,643 & 3,034 & 4,00 \\
SBA & 350,590 & 6,00 & 0,206 & 1,058 & 4,489 & 6,00 \\
CROSTON & 388,999 & 7,00 & 0,270 & 1,445 & 5,841 & 7,00 \\
SBJ & 348,569 & 5,00 & 0,202 & 1,038 & 4,417 & 5,00 \\
SES & $-23,879$ & 3,00 & $-0,005$ & $-0,204$ & 0,072 & 1,33 \\
ZERO & $-529,579$ & 8,00 & $-1,018$ & $-6,285$ & $-21,215$ & 8,00 \\
NAIVE & $-3,493$ & 2,00 & 0,082 & 0,318 & 1,896 & 3,00 \\
TSB & $-27,535$ & 4,00 & $-0,010$ & $-0,233$ & $-0,032$ & 1,67
\end{tabular}

\begin{tabular}{|c|c|c|c|c|c|c|}
\hline TAME & Treino & & & Teste & & \\
\hline Modelo & $\begin{array}{c}\text { a } 1 \text { passo } \\
32 \mathrm{p} .\end{array}$ & $\begin{array}{l}\text { Ranking } \\
\text { treino }\end{array}$ & $T+1$ & $T+3$ & & $\begin{array}{c}\text { Rankin } \\
\text { teste }\end{array}$ \\
\hline MMS3 & 27,562 & 6,00 & 1,189 & 3,562 & 7,119 & 7,00 \\
\hline SBA & 22,294 & 4,00 & 0,433 & 1,325 & 2,731 & 3,00 \\
\hline CROSTON & 23,824 & 5,00 & 0,463 & 1,416 & 2,917 & 4,00 \\
\hline SBJ & 22,217 & 3,00 & 0,431 & 1,321 & 2,723 & 2,00 \\
\hline SES & 12,976 & 1,00 & 0,319 & 0,976 & 1,999 & 1,00 \\
\hline ZERO & 31,993 & 7,00 & 1,001 & 3,008 & 6,006 & 6,00 \\
\hline NAIIVE & 43,222 & 8,00 & 1,575 & 4,724 & 9,434 & 8,00 \\
\hline TSB & 14,861 & 2,00 & 0,519 & 1,562 & 3,144 & 5,0 \\
\hline
\end{tabular}

$\begin{array}{lcccccc}\text { TQME } & \begin{array}{c}\text { Treino } \\ \text { a 1 passo } \\ \text { Modelo }\end{array} & \begin{array}{c}\text { Ranking } \\ \text { treino }\end{array} & \mathrm{T}+1 & \mathrm{~T}+3 & \mathrm{~T}+6 & \begin{array}{c}\text { Ranking } \\ \text { teste }\end{array} \\ \text { MMS3 } & 51,735 & 4,00 & 2,805 & 8,418 & 16,940 & 7,00 \\ \text { SBA } & 82,971 & 6,00 & 0,539 & 1,661 & 3,489 & 3,33 \\ \text { CROSTON } & 94,273 & 7,00 & 0,622 & 1,909 & 3,988 & 5,00 \\ \text { SBJ } & 82,402 & 5,00 & 0,535 & 1,648 & 3,464 & 2,33 \\ \text { SES } & 21,527 & 2,00 & 0,180 & 0,577 & 1,285 & 1,00 \\ \text { ZERO } & 51,459 & 3,00 & 1,022 & 3,130 & 6,393 & 6,00 \\ \text { NAïV } & 191,139 & 8,00 & 7,986 & 23,946 & 47,935 & 8,00 \\ \text { TSB } & 19,043 & 1,00 & 0,550 & 1,676 & 3,455 & 3,33\end{array}$

$\begin{array}{lcccccc}\text { PEEEAm } & \begin{array}{c}\text { Treino } \\ \text { a 1 passo } \\ \text { Modelo }\end{array} & \begin{array}{c}\text { Ranking } \\ \text { treino }\end{array} & \mathrm{T}+1 & \mathrm{~T}+3 & \mathrm{~T}+6 & \begin{array}{c}\text { Ranking } \\ \text { teste }\end{array} \\ \text { MMS3 } & 67,449 & 2,00 & 1,691 & 8,886 & 28,956 & 5,00 \\ \text { SBA } & 499,495 & 6,00 & 1,806 & 9,040 & 27,692 & 6,00 \\ \text { CROSTON } & 527,086 & 7,00 & 1,851 & 9,255 & 28,391 & 7,00 \\ \text { SBJ } & 498,071 & 5,00 & 1,804 & 9,029 & 27,656 & 5,00 \\ \text { SES } & 320,406 & 4,00 & 1,593 & 7,889 & 23,634 & 2,33 \\ \text { ZERO } & 529,579 & 8,00 & 1,018 & 6,285 & 21,215 & 1,00 \\ \text { NAïV } & 44,607 & 1,00 & 1,751 & 9,953 & 33,625 & 7,00 \\ \text { TSB } & 231,558 & 3,00 & 1,587 & 7,907 & 23,979 & 2,67\end{array}$

NÃO OTIMIZADO (INÍCIO Pt = MÉDIA )

\begin{tabular}{|c|c|c|c|c|c|c|}
\hline DMAE & Treino & & & Teste & & \\
\hline Modelo & $\begin{array}{c}\text { a } 1 \text { passo } \\
32 \mathrm{p} .\end{array}$ & $\begin{array}{c}\text { Ranking } \\
\text { treino }\end{array}$ & $T+1$ & $T+3$ & $T+6$ & $\begin{array}{r}\text { Rank } \\
\text { tes }\end{array}$ \\
\hline MMS3 & 1,560 & 6,00 & 1,691 & 1,706 & 1,703 & 70 \\
\hline SBA & 1,533 & 5,00 & 1,635 & 1,641 & 1,624 & 0 \\
\hline CROSTON & 1,567 & 7,00 & 1,670 & 1,676 & 1,660 & . \\
\hline SBJ & 1,531 & 4,00 & 1,633 & 1,639 & 1,622 & 0 \\
\hline SES & 1,521 & 3,00 & 1,592 & 1,608 & 1,591 & $3, \mathrm{C}$ \\
\hline ZERO & 1,000 & 1,00 & 1,018 & 1,039 & 0,992 & 1,0 \\
\hline NAIIVE & 1,629 & 8,00 & 1,751 & 1,744 & 1,723 & 8, \\
\hline TSB & 1,512 & 2,00 & 1,587 & 1,592 & 1,585 & \\
\hline
\end{tabular}

\begin{tabular}{lcccccc} 
DQME & \multicolumn{1}{c}{ Treino } & \multicolumn{5}{c}{ Teste } \\
Modelo & $\begin{array}{c}\text { 1 passo } \\
32 \mathrm{p} .\end{array}$ & $\begin{array}{c}\text { Ranking } \\
\text { treino }\end{array}$ & $\mathrm{T}+1$ & $\mathrm{~T}+3$ & $\mathrm{~T}+6$ & $\begin{array}{c}\text { Ranking } \\
\text { teste }\end{array}$ \\
MMS3 & 9,175 & 7,00 & 25,088 & 20,735 & 20,077 & 7,00 \\
SBA & 6,597 & 2,00 & 22,751 & 18,482 & 17,678 & 3,00 \\
CROSTON & 6,646 & 3,00 & 22,768 & 18,495 & 17,697 & 4,00 \\
SBJ & 6,594 & 1,00 & 22,750 & 18,482 & 17,677 & 2,00 \\
SES & 7,171 & 4,00 & 22,605 & 18,409 & 17,600 & 1,00 \\
ZERO & 7,689 & 6,00 & 23,662 & 19,471 & 18,557 & 6,00 \\
NAIVE & 13,554 & 8,00 & 30,696 & 25,934 & 25,042 & 8,00 \\
TSB & 7,609 & 5,00 & 22,985 & 18,673 & 17,897 & 5,00
\end{tabular}

\begin{tabular}{lcccccc} 
EMAE & Treino & \multicolumn{5}{c}{ Teste } \\
Modelo & $\begin{array}{c}\text { a 1 passo } \\
\text { 32 p. }\end{array}$ & $\begin{array}{c}\text { Ranking } \\
\text { treino }\end{array}$ & $\mathrm{T}+1$ & $\mathrm{~T}+3$ & $\mathrm{~T}+6$ & $\begin{array}{c}\text { Ranking } \\
\text { teste }\end{array}$ \\
MMS3 & 0,962 & 5,00 & 1,048 & 1,062 & 1,061 & 7,00 \\
SBA & 0,963 & 6,00 & 1,004 & 1,014 & 1,005 & 5,00 \\
CROSTON & 0,984 & 7,00 & 1,026 & 1,035 & 1,027 & 6,00 \\
SBJ & 0,962 & 4,00 & 1,003 & 1,013 & 1,004 & 4,00 \\
SES & 0,947 & 3,00 & 0,976 & 0,991 & 0,982 & 3,00 \\
ZERO & 0,649 & 1,00 & 0,636 & 0,656 & 0,627 & 1,00 \\
NAIVE & 1,000 & 8,00 & 1,142 & 1,142 & 1,131 & 8,00 \\
TSB & 0,939 & 2,00 & 0,976 & 0,983 & 0,980 & 2,00
\end{tabular}

\begin{tabular}{lcccccc} 
PEEE & \multicolumn{2}{c}{ Treino } & \multicolumn{5}{c}{ Teste } \\
Modelo & $\begin{array}{c}\text { 1 passo } \\
32 \mathrm{p} .\end{array}$ & $\begin{array}{c}\text { Ranking } \\
\text { treino }\end{array}$ & $\mathrm{T}+1$ & $\mathrm{~T}+3$ & $\mathrm{~T}+6$ & $\begin{array}{c}\text { Ranking } \\
\text { teste }\end{array}$ \\
MMS3 & $-2,898$ & 2,00 & 0,136 & 0,643 & 3,034 & 7,00 \\
SBA & 40,409 & 6,00 & 0,019 & $-0,063$ & 0,564 & 3,00 \\
CROSTON & 62,493 & 7,00 & 0,073 & 0,265 & 1,710 & 5,00 \\
SBJ & 39,247 & 5,00 & 0,016 & $-0,080$ & 0,504 & 2,67 \\
SES & 1,565 & 1,00 & $-0,001$ & $-0,181$ & 0,150 & 2,00 \\
ZERO & $-529,579$ & 8,00 & $-1,018$ & $-6,285$ & $-21,215$ & 8,00 \\
NAIVE & $-3,493$ & 3,00 & 0,082 & 0,318 & 1,896 & 6,00 \\
TSB & $-22,835$ & 4,00 & $-0,010$ & $-0,233$ & $-0,031$ & 2,33
\end{tabular}

$\begin{array}{lcccccc}\text { TAME } & \begin{array}{c}\text { Treino } \\ \text { a 1 passo }\end{array} & \begin{array}{c}\text { Ranking } \\ \text { Modelo } \\ \text { treino }\end{array} & \begin{array}{c}\mathrm{T}+1 \\ \mathrm{p} .\end{array} & \mathrm{T}+3 & \mathrm{~T}+6 & \begin{array}{c}\text { Ranking } \\ \text { teste }\end{array} \\ \text { MMS3 } & 27,562 & 6,00 & 1,189 & 3,562 & 7,119 & 7,00 \\ \text { SBA } & 10,022 & 2,00 & 0,244 & 0,763 & 1,616 & 2,00 \\ \text { CROSTON } & 10,270 & 3,00 & 0,259 & 0,807 & 1,710 & 3,00 \\ \text { SBJ } & 10,013 & 1,00 & 0,243 & 0,761 & 1,612 & 1,00 \\ \text { SES } & 11,820 & 4,00 & 0,319 & 0,977 & 2,002 & 4,00 \\ \text { ZERO } & 31,993 & 7,00 & 1,001 & 3,008 & 6,006 & 6,00 \\ \text { NAIIVE } & 43,222 & 8,00 & 1,575 & 4,724 & 9,434 & 8,00 \\ \text { TSB } & 15,693 & 5,00 & 0,519 & 1,562 & 3,145 & 5,00\end{array}$

\begin{tabular}{lcccccc} 
TQME & Treino & \multicolumn{5}{c}{ Teste } \\
Modelo & $\begin{array}{c}\text { a 1 passo } \\
32 \mathrm{p} .\end{array}$ & $\begin{array}{c}\text { Ranking } \\
\text { treino }\end{array}$ & $\mathrm{T}+1$ & $\mathrm{~T}+3$ & $\mathrm{~T}+6$ & $\begin{array}{c}\text { Ranking } \\
\text { teste }\end{array}$ \\
MMS3 & 51,735 & 7,00 & 2,805 & 8,418 & 16,940 & 7,00 \\
SBA & 9,187 & 2,00 & 0,125 & 0,421 & 0,995 & 2,00 \\
CROSTON & 9,511 & 3,00 & 0,143 & 0,475 & 1,103 & 3,00 \\
SBJ & 9,176 & 1,00 & 0,124 & 0,419 & 0,991 & 1,00 \\
SES & 14,265 & 4,00 & 0,181 & 0,583 & 1,295 & 4,00 \\
ZERO & 51,459 & 6,00 & 1,022 & 3,130 & 6,393 & 6,00 \\
NAIIVE & 191,139 & 8,00 & 7,986 & 23,946 & 47,935 & 8,00 \\
TSB & 16,912 & 5,00 & 0,551 & 1,677 & 3,457 & 5,00
\end{tabular}

\begin{tabular}{|c|c|c|c|c|c|c|}
\hline EEEAm & Treino & & & Teste & & \\
\hline Modelo & $\begin{array}{c}\text { a } 1 \text { passo } \\
32 \mathrm{p} .\end{array}$ & $\begin{array}{c}\text { Ranking } \\
\text { treino }\end{array}$ & $T+1$ & $\mathrm{~T}+3$ & $T+6$ & $\begin{array}{c}\text { Ranking } \\
\text { teste }\end{array}$ \\
\hline MMS3 & 67,449 & 3,00 & 1,691 & 8,886 & 28,956 & 7,00 \\
\hline SBA & 194,054 & 6,00 & 1,635 & 8,129 & 24,614 & 5,00 \\
\hline CROSTON & 199,952 & 7,00 & 1,670 & 8,286 & 25,115 & 6,00 \\
\hline SBJ & 193,782 & 5,00 & 1,633 & 8,121 & 24,589 & 4,00 \\
\hline SES & 28,567 & 1,00 & 1,592 & 7,881 & 23,624 & 2,33 \\
\hline ZERO & 529,579 & 8,00 & 1,018 & 6,285 & 21,215 & 1,00 \\
\hline NAIIVE & 44,607 & 2,00 & 1,751 & 9,953 & 33,625 & 8,00 \\
\hline TSB & 154,241 & 4,00 & 1,587 & 7,907 & 23,979 & 2,67 \\
\hline
\end{tabular}




\section{APÊNDICE C - Classificação de erros de otimizações (demanda alto giro).}

\section{DMAE DESVIO MÉDIO ABSOLUTO ESCALONADO}

\begin{tabular}{lcccccc} 
MMS3 & \multicolumn{2}{c}{ Treino } & \multicolumn{3}{c}{ Teste } \\
Otimização & $\begin{array}{c}\text { 1 1 passo Ranking } \\
\text { 32 p. }\end{array}$ & $\begin{array}{c}\text { treino } \\
\text { Tr1 }\end{array}$ & T+3 & T+6 & $\begin{array}{c}\text { Ranking } \\
\text { teste }\end{array}$ \\
OT DMA & 0,7068 & 3,50 & 0,5604 & 0,6186 & 0,6495 & 3,50 \\
OT DQM & 0,7068 & 3,50 & 0,5604 & 0,6186 & 0,6495 & 3,50 \\
OT TAM & 0,7068 & 3,50 & 0,5604 & 0,6186 & 0,6495 & 3,50 \\
OT TQM & 0,7068 & 3,50 & 0,5604 & 0,6186 & 0,6495 & 3,50 \\
NOT naive & 0,7068 & 3,50 & 0,5604 & 0,6186 & 0,6495 & 3,50 \\
NOT média & 0,7068 & 3,50 & 0,5604 & 0,6186 & 0,6495 & 3,50
\end{tabular}

\begin{tabular}{lllccc} 
SBA & Treino & \multicolumn{3}{c}{ Teste } \\
Otimização & $\begin{array}{c}\text { a 1 passo Ranking } \\
32 \mathrm{p} \text {. }\end{array} \quad \mathrm{treino}$ & $\mathrm{T}+1$ & $\mathrm{~T}+3$ & $\mathrm{~T}+6$ & $\begin{array}{c}\text { Ranking } \\
\text { teste }\end{array}$
\end{tabular} $\begin{array}{lllllll}\text { OT DMA } & 0,6000 & 1,00 & 0,5393 & 0,5809 & 0,6096 & 1,00\end{array}$ $\begin{array}{llllllll}\text { OT DQM } & 0,6250 & 2,00 & 0,6187 & 0,6546 & 0,6834 & 2,00\end{array}$ $\begin{array}{llllllll}\text { OT TAM } & 0,6794 & 4,00 & 0,6838 & 0,7102 & 0,7355 & 5,33\end{array}$ $\begin{array}{llllllll}\text { OT TQM } & 0,6777 & 3,00 & 0,6842 & 0,7085 & 0,7364 & 5,67\end{array}$ $\begin{array}{llllllll}\text { NOT naive } & 0,7001 & 6,00 & 0,6458 & 0,6711 & 0,6995 & 4,00\end{array}$

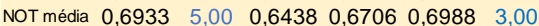

CROSTON Treino Teste

Otimização $\begin{gathered}\text { a } 1 \text { passo Ranking } \\ 32 \mathrm{p} .\end{gathered} \quad \begin{gathered}\text { treino } \\ \mathrm{T}+1\end{gathered} \quad \mathrm{~T}+3 \quad \mathrm{~T}+6 \begin{gathered}\begin{array}{c}\text { Ranking } \\ \text { teste }\end{array} \\ 0.609\end{gathered}$ $\begin{array}{lllllll}\text { OT DMA } & 0,6090 & 1,00 & 0,5594 & 0,5998 & 0,6240 & 1,00\end{array}$ $\begin{array}{llllllll}\text { OT DQM } & 0,6349 & 2,00 & 0,6306 & 0,6692 & 0,6994 & 2,00\end{array}$ $\begin{array}{llllllll}\text { От TAM } & 0,6817 & 4,00 & 0,6876 & 0,7123 & 0,7408 & 5,00\end{array}$ $\begin{array}{llllllll}\text { От TQM } & 0,6808 & 3,00 & 0,6918 & 0,7157 & 0,7426 & 6,00\end{array}$ $\begin{array}{llllllll}\text { NOT naïve } & 0,7123 & 6,00 & 0,6715 & 0,6922 & 0,7195 & 4,00\end{array}$ NOT média $0,7046 \quad 5,00 \quad 0,6691 \quad 0,6918 \quad 0,7189 \quad 3,00$

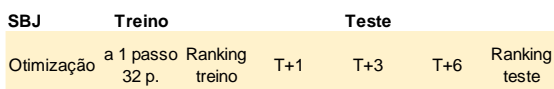

$\begin{array}{llllllll}\text { OT DMA } & 0,5988 & 1,00 & 0,5407 & 0,5838 & 0,6095 & 1,00\end{array}$ $\begin{array}{llllllll}\text { OT DQM } & 0,6251 & 2,00 & 0,6139 & 0,6501 & 0,6793 & 2,00\end{array}$ $\begin{array}{lllllllll}\text { OT TAM } & 0,6807 & 4,00 & 0,6792 & 0,7091 & 0,7364 & 5,00\end{array}$ $\begin{array}{lllllll}\text { От TQM } & 0,6781 & 3,00 & 0,6835 & 0,7099 & 0,7380 & 6,00\end{array}$ NOT naiive $\quad 0,6995 \quad 6,00 \quad 0,64450,6700 \quad 0,6985 \quad 4,00$ $\begin{array}{lllllll}\text { NOT média } & 0,6928 & 5,00 & 0,6425 & 0,6696 & 0,6978 & 3,00\end{array}$

\begin{tabular}{|c|c|c|}
\hline & Treino & Teste \\
\hline
\end{tabular}

$\begin{array}{lllllllllll}\text { OT DMA } & 0,6031 & 1,00 & 0,5241 & 0,5667 & 0,5924 & 1,00\end{array}$ $\begin{array}{lllllll}\text { OT DQM } & 0,6314 & 2,00 & 0,5960 & 0,6253 & 0,6515 & 2,00\end{array}$ $\begin{array}{llllllll}\text { Oт TAM } & 0,6722 & 4,00 & 0,6692 & 0,6890 & 0,7168 & 5,00\end{array}$ $\begin{array}{lllllllll}\text { От TQM } & 0,6730 & 5,00 & 0,6717 & 0,6909 & 0,7183 & 6,00\end{array}$ $\begin{array}{lllllll}\text { NOT naiive } & 0,6779 & 6,00 & 0,6106 & 0,6287 & 0,6571 & 4,00\end{array}$ $\begin{array}{lllllll}\text { NOT média } & 0,6605 & 3,00 & 0,6104 & 0,6286 & 0,6568 & 3,00\end{array}$

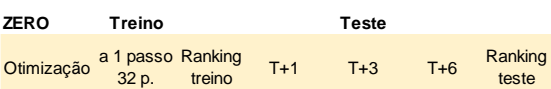

$\begin{array}{lllllll}\text { OT DMA } & 1,0000 & 3,50 & 0,6938 & 0,7638 & 0,7857 & 3,50\end{array}$ $\begin{array}{lllllll}\text { OT DQM } & 1,0000 & 3,50 & 0,6938 & 0,7638 & 0,7857 & 3,50\end{array}$ $\begin{array}{llllllll}\text { Oт TAM } & 1,0000 & 3,50 & 0,6938 & 0,7638 & 0,7857 & 3,50\end{array}$ $\begin{array}{llllllll}\text { OT TQM } & 1,0000 & 3,50 & 0,6938 & 0,7638 & 0,7857 & 3,50\end{array}$ $\begin{array}{lllllll}\text { NOT naive } & 1,0000 & 3,50 & 0,6938 & 0,7638 & 0,7857 & 3,50\end{array}$ $\begin{array}{lllllll}\text { NOT média } & 1,0000 & 3,50 & 0,6938 & 0,7638 & 0,7857 & 3,50\end{array}$

$\begin{array}{llcccc}\text { NAIVE } & \text { Treino } & \text { Teste } & & \\ \text { Otimização a 1 passo Ranking } & \mathrm{T} & \mathrm{t}+1 & \mathrm{~T}+3 & \mathrm{~T}+6 & \begin{array}{c}\text { Ranking } \\ \text { teste }\end{array}\end{array}$

$\begin{array}{llllllll}\text { OT DMA } & 0,8317 & 3,50 & 0,6337 & 0,6914 & 0,7185 & 3,50\end{array}$ $\begin{array}{llllllll}\text { OT DQM } & 0,8317 & 3,50 & 0,6337 & 0,6914 & 0,7185 & 3,50\end{array}$ $\begin{array}{lllllllll}\text { OT TAM } & 0,8317 & 3,50 & 0,6337 & 0,6914 & 0,7185 & 3,50\end{array}$ $\begin{array}{llllllll}\text { OT TQM } & 0,8317 & 3,50 & 0,6337 & 0,6914 & 0,7185 & 3,50\end{array}$ $\begin{array}{llllllll}\text { NOT naïv } & 0,8317 & 3,50 & 0,6337 & 0,6914 & 0,7185 & 3,50\end{array}$ $\begin{array}{lllllll}\text { NOT média } & 0,8317 & 3,50 & 0,6337 & 0,6914 & 0,7185 & 3,50\end{array}$

$\begin{array}{llcccc}\text { TSB } & \text { Treino } & \text { Teste } & & \\ \text { Otimização a 1 passo Ranking } & & & & & \\ 32 \mathrm{p} \text { treino } & \mathrm{T}+1 & \mathrm{~T}+3 & \mathrm{~T}+6 & \begin{array}{c}\text { Ranking } \\ \text { teste }\end{array}\end{array}$

$\begin{array}{lllllll}\text { OT DMA } & 0,6022 & 1,00 & 0,5366 & 0,5739 & 0,5997 & 1,00\end{array}$ $\begin{array}{lllllll}\text { Oт DQM } & 0,6271 & 2,00 & 0,5943 & 0,6278 & 0,6573 & 4,00\end{array}$ $\begin{array}{llllllll}\text { OT TAM } & 0,6771 & 4,00 & 0,6692 & 0,6919 & 0,7178 & 5,33\end{array}$ $\begin{array}{lllllll}\text { OT TQM } & 0,6760 & 3,00 & 0,6705 & 0,6911 & 0,7179 & 5,67\end{array}$ $\begin{array}{lllllll}\text { NOT naïve } & 0,6915 & 6,00 & 0,5786 & 0,5980 & 0,6265 & 3,00\end{array}$ $\begin{array}{lllllll}\text { NOT média } & 0,6868 & 5,00 & 0,5786 & 0,5980 & 0,6265 & 2,00\end{array}$

\section{EMAE ERRO MÉDIO ABSOLUTO ESCALONADO}

\begin{tabular}{lcccccc} 
MMS3 & \multicolumn{2}{c}{ Treino } & \multicolumn{2}{c}{ Teste } \\
Otimização & $\begin{array}{c}\text { 1 1 passo } \\
32 \mathrm{p} \text {. }\end{array}$ & $\begin{array}{c}\text { Ranking } \\
\text { treino }\end{array}$ & $\mathrm{T}+1$ & $\mathrm{~T}+3$ & $\mathrm{~T}+6$ & $\begin{array}{c}\text { Ranking } \\
\text { teste }\end{array}$ \\
OT DMA & 0,8531 & 3,50 & 0,6921 & 0,7541 & 0,7956 & 3,50 \\
OT DQM & 0,8531 & 3,50 & 0,6921 & 0,7541 & 0,7956 & 3,50 \\
OT TAM & 0,8531 & 3,50 & 0,6921 & 0,7541 & 0,7956 & 3,50 \\
OT TQM & 0,8531 & 3,50 & 0,6921 & 0,7541 & 0,7956 & 3,50 \\
NOT naïe & 0,8531 & 3,50 & 0,6921 & 0,7541 & 0,7956 & 3,50 \\
NOT média & 0,8531 & 3,50 & 0,6921 & 0,7541 & 0,7956 & 3,50
\end{tabular}

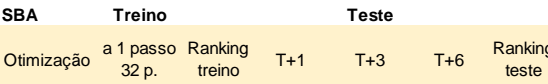

$\begin{array}{lllllll}\text { Oт DMA } & 0,7334 & 1,00 & 0,6654 & 0,7091 & 0,7506 & 1,00\end{array}$

$\begin{array}{llllllll}\text { OT DQM } & 0,7601 & 2,00 & 0,7562 & 0,7933 & 0,8344 & 2,00\end{array}$

$\begin{array}{llllllllll}\text { От TAM } & 0,8262 & 4,00 & 0,8396 & 0,8617 & 0,8985 & 6,00\end{array}$

$\begin{array}{lllllllll}\text { OT TQM } & 0,8238 & 3,00 & 0,8381 & 0,8578 & 0,8981 & 5,00\end{array}$

$\begin{array}{llllllll}\text { NOT naîve } & 0,8530 & 6,00 & 0,7906 & 0,8119 & 0,8539 & 4,00\end{array}$

$\begin{array}{lllllll}\text { NOT média } & 0,8451 & 5,00 & 0,7884 & 0,8112 & 0,8529 & 3,00\end{array}$

CROSTON Trein

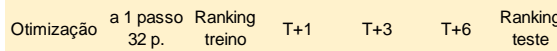

$\begin{array}{lllllll}\text { Oт DMA } & 0,7455 & 1,00 & 0,6931 & 0,7350 & 0,7713 & 1,00\end{array}$

$\begin{array}{llllllll}\text { OT DQM } & 0,7731 & 2,00 & 0,7740 & 0,8130 & 0,8552 & 2,00\end{array}$

$\begin{array}{lllllllll}\text { от тAM } & 0,8295 & 4,00 & 0,8458 & 0,8651 & 0,9068 & 5,00\end{array}$

$\begin{array}{llllllll}\text { Oт TQM } & 0,8284 & 3,00 & 0,8505 & 0,8693 & 0,9094 & 6,00\end{array}$

$\begin{array}{lllllll}\text { NOT naive } & 0,8668 & 6,00 & 0,8239 & 0,8393 & 0,8799 & 4,00\end{array}$

$\begin{array}{lllllll}\text { NOT média } & 0,8578 & 5,00 & 0,8214 & 0,8385 & 0,8790 & 3,00\end{array}$

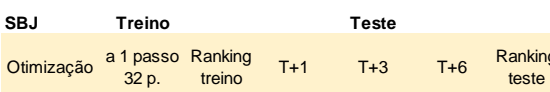

$\begin{array}{lllllll}\text { Oт DMA } & 0,7320 & 1,00 & 0,6670 & 0,7137 & 0,7510 & 1,00\end{array}$

$\begin{array}{llllllll}\text { OT DQM } & 0,7605 & 2,00 & 0,7513 & 0,7888 & 0,8300 & 2,00\end{array}$

$\begin{array}{lllllllll}\text { OT TAM } & 0,8277 & 4,00 & 0,8329 & 0,8591 & 0,8991 & 5,00\end{array}$

$\begin{array}{llllllll}\text { От TQM } & 0,8246 & 3,00 & 0,8393 & 0,8617 & 0,9018 & 6,00\end{array}$

$\begin{array}{llllllll}\text { NOT naïe } & 0,8523 & 6,00 & 0,7889 & 0,8105 & 0,8525 & 4,00\end{array}$

$\begin{array}{llllllll}\text { NOT média } & 0,8446 & 5,00 & 0,7867 & 0,8098 & 0,8516 & 3,00\end{array}$

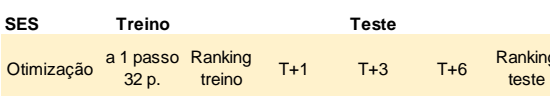

$\begin{array}{llllllll}\text { OT DMA } & 0,7340 & 1,00 & 0,6485 & 0,6939 & 0,7321 & 1,00\end{array}$

$\begin{array}{llllllll}\text { OT DQM } & 0,7655 & 2,00 & 0,7329 & 0,7599 & 0,7975 & 2,00\end{array}$

$\begin{array}{llllllllll}\text { от тAM } & 0,8166 & 4,00 & 0,8271 & 0,8389 & 0,8804 & 5,00\end{array}$

$\begin{array}{llllllll}\text { OT TQM } & 0,8175 & 5,00 & 0,8301 & 0,8411 & 0,8822 & 6,00\end{array}$

$\begin{array}{llllllll}\text { NOT naive } & 0,8256 & 6,00 & 0,7558 & 0,7678 & 0,8096 & 4,00\end{array}$

$\begin{array}{lllllll}\text { NOT média } & 0,8019 & 3,00 & 0,7547 & 0,7674 & 0,8089 & 3,00\end{array}$

ZERO Treino Teste

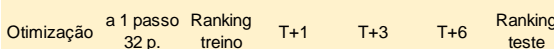

$\begin{array}{lllllll}\text { OT DMA } & 1,3204 & 3,50 & 0,9303 & 1,0041 & 1,0379 & 3,50\end{array}$

$\begin{array}{llllllll}\text { OT DQM } & 1,3204 & 3,50 & 0,9303 & 1,0041 & 1,0379 & 3,50\end{array}$

$\begin{array}{lllllllll}\text { OT TAM } & 1,3204 & 3,50 & 0,9303 & 1,0041 & 1,0379 & 3,50\end{array}$

$\begin{array}{llllllll}\text { OT TQM } & 1,3204 & 3,50 & 0,9303 & 1,0041 & 1,0379 & 3,50\end{array}$

NOT naïe $\begin{array}{lllllll}1,3204 & 3,50 & 0,9303 & 1,0041 & 1,0379 & 3,50\end{array}$

NOT média $\begin{array}{lllllll}1,3204 & 3,50 & 0,9303 & 1,0041 & 1,0379 & 3,50\end{array}$

$\begin{array}{llcccc}\text { NAIVE } & \text { Treino } & \text { Teste } & \\ \text { Otimização } \begin{array}{c}\text { a } 1 \text { passo } \\ 32 \mathrm{p} .\end{array} & \begin{array}{c}\text { Ranking } \\ \text { treino }\end{array} & \mathrm{T}+1 & \mathrm{~T}+3 & \mathrm{~T}+6 & \begin{array}{c}\text { Ranking } \\ \text { teste }\end{array}\end{array}$

$\begin{array}{lllllll}\text { Oт DMA } & 1,0000 & 3,50 & 0,7899 & 0,8495 & 0,8871 & 3,50\end{array}$

$\begin{array}{llllllll}\text { OT DQM } & 1,0000 & 3,50 & 0,7899 & 0,8495 & 0,8871 & 3,50\end{array}$

$\begin{array}{llllllll}\text { От TAM } & 1,0000 & 3,50 & 0,7899 & 0,8495 & 0,8871 & 3,50\end{array}$

$\begin{array}{llllllll}\text { OT TQM } & 1,0000 & 3,50 & 0,7899 & 0,8495 & 0,8871 & 3,50\end{array}$

$\begin{array}{lllllll}\text { NOT naive } & 1,0000 & 3,50 & 0,7899 & 0,8495 & 0,8871 & 3,50\end{array}$

$\begin{array}{lllllll}\text { NOT média } & 1,0000 & 3,50 & 0,7899 & 0,8495 & 0,8871 & 3,50\end{array}$

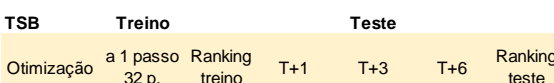

$\begin{array}{llllllll}\text { OT DMA } & 0,7339 & 1,00 & 0,6605 & 0,7002 & 0,7381 & 1,00\end{array}$

$\begin{array}{llllllll}\text { OT DQM } & 0,7616 & 2,00 & 0,7272 & 0,7605 & 0,8032 & 4,00\end{array}$

$\begin{array}{llllllll}\text { OT TAM } & 0,8237 & 4,00 & 0,8244 & 0,8415 & 0,8805 & 5,67\end{array}$

$\begin{array}{lllllllll}\text { OT TQM } & 0,8214 & 3,00 & 0,8255 & 0,8395 & 0,8796 & 5,33\end{array}$

$\begin{array}{llllllll}\text { NOT naive } & 0,8429 & 6,00 & 0,7201 & 0,7331 & 0,7748 & 3,00\end{array}$

$\begin{array}{lllllll}\text { NOT média } & 0,8376 & 5,00 & 0,7201 & 0,7330 & 0,7748 & 2,00\end{array}$ 


$\begin{array}{lccccccc}\text { MMS3 } & \begin{array}{c}\text { Treino } \\ \text { Otimização }\end{array} & \begin{array}{c}\text { 1 passo } \\ 32 \mathrm{p} .\end{array} & \begin{array}{c}\text { Ranking } \\ \text { treino }\end{array} & \begin{array}{c}\text { Teste } \\ \text { T+1 }\end{array} & \mathrm{T}+3 & \mathrm{~T}+6 & \begin{array}{c}\text { Ranking } \\ \text { teste }\end{array} \\ \text { OT DMA } & 1,0033 & 3,50 & 0,6456 & 0,9079 & 1,0281 & 3,50 \\ \text { OT DQM } & 1,0033 & 3,50 & 0,6456 & 0,9079 & 1,0281 & 3,50 \\ \text { OT TAM } & 1,0033 & 3,50 & 0,6456 & 0,9079 & 1,0281 & 3,50 \\ \text { OT TQM } & 1,0033 & 3,50 & 0,6456 & 0,9079 & 1,0281 & 3,50 \\ \text { NOT naïve } & 1,0033 & 3,50 & 0,6456 & 0,9079 & 1,0281 & 3,50 \\ \text { NOT média } & 1,0033 & 3,50 & 0,6456 & 0,9079 & 1,0281 & 3,50\end{array}$

$\begin{array}{lccccccc}\text { SBA } & \begin{array}{c}\text { Treino } \\ \text { Otimização }\end{array} & \begin{array}{c}\text { 1 passo } \\ 32 \mathrm{p} .\end{array} & \begin{array}{c}\text { Ranking } \\ \text { treino }\end{array} & \mathrm{T}+1 & \mathrm{~T}+3 & \mathrm{~T}+6 & \begin{array}{r}\text { Rankin } \\ \text { teste }\end{array} \\ \text { OT DMA } & 0,8512 & 2,00 & 0,5595 & 0,7733 & 0,8715 & 1,00 \\ \text { OT DQM } & 0,7771 & 1,00 & 0,7634 & 0,9733 & 1,0829 & 6,00 \\ \text { OT TAM } & 0,8662 & 4,00 & 0,7173 & 0,8810 & 0,9731 & 5,00 \\ \text { OT TQM } & 0,8580 & 3,00 & 0,7109 & 0,8741 & 0,9709 & 4,00 \\ \text { NOT naïve } & 0,9404 & 6,00 & 0,6646 & 0,8272 & 0,9243 & 2,67 \\ \text { NOT média } & 0,9233 & 5,00 & 0,6610 & 0,8266 & 0,9244 & 2,33\end{array}$

$\begin{array}{lllllll}0,9233 & 5,00 & 0,6610 & 0,8266 & 0,9244 & 2,33\end{array}$

$\begin{array}{lccccccc}\text { CROSTON } & \begin{array}{c}\text { Treino } \\ \text { Otimização } \\ \text { a 1 passo } \\ 32 \mathrm{p} \text {. }\end{array} & \begin{array}{c}\text { Ranking } \\ \text { treino }\end{array} & \mathrm{T}+1 & \mathrm{~T}+3 & \mathrm{~T}+6 & \begin{array}{c}\text { Ranking } \\ \text { teste }\end{array} \\ \text { OT DMA } & 0,8522 & 2,00 & 0,5697 & 0,7843 & 0,8775 & 1,00 \\ \text { OT DQM } & 0,7849 & 1,00 & 0,7792 & 0,9915 & 1,1006 & 6,00 \\ \text { OT TAM } & 0,8694 & 4,00 & 0,7184 & 0,8823 & 0,9814 & 4,00 \\ \text { OT TQM } & 0,8634 & 3,00 & 0,7318 & 0,8878 & 0,9838 & 5,00 \\ \text { NOT naïv } & 0,9559 & 6,00 & 0,6934 & 0,8495 & 0,9447 & 2,67 \\ \text { NOT média } & 0,9349 & 5,00 & 0,6896 & 0,8489 & 0,9447 & 2,33\end{array}$

$\begin{array}{lccccccc}\text { SBJ } & \begin{array}{c}\text { Treino } \\ \text { Otimização }\end{array} \begin{array}{c}\text { a 1 passo } \\ 32 \mathrm{p} .\end{array} & \begin{array}{c}\text { Ranking } \\ \text { treino }\end{array} & \mathrm{T}+1 & \mathrm{~T}+3 & \mathrm{~T}+6 & \begin{array}{c}\text { Ranking } \\ \text { teste }\end{array} \\ \text { OT DMA } & 0,8547 & 2,00 & 0,5562 & 0,7743 & 0,8752 & 1,00 \\ \text { OT DQM } & 0,7808 & 1,00 & 0,7600 & 0,9675 & 1,0768 & 6,00 \\ \text { OT TAM } & 0,8702 & 4,00 & 0,7157 & 0,8830 & 0,9767 & 4,67 \\ \text { OT TQM } & 0,8588 & 3,00 & 0,7157 & 0,8760 & 0,9754 & 4,33 \\ \text { NOT naïve } & 0,9397 & 6,00 & 0,6632 & 0,8261 & 0,9234 & 2,67 \\ \text { NOT média } & 0,9228 & 5,00 & 0,6596 & 0,8255 & 0,9235 & 2,33\end{array}$

$\begin{array}{lccccccc}\text { SES } & \begin{array}{c}\text { Treino } \\ \text { Otimização }\end{array} \begin{array}{c}\text { a 1 passo } \\ 32 \mathrm{p} .\end{array} & \begin{array}{c}\text { Ranking } \\ \text { treino }\end{array} & \mathrm{T}+1 & \mathrm{~T}+3 & \mathrm{~T}+6 & \begin{array}{c}\text { Ranking } \\ \text { teste }\end{array} \\ \text { OT DMA } & 0,8358 & 5,00 & 0,5505 & 0,7620 & 0,8540 & 2,33 \\ \text { OT DQM } & 0,7707 & 1,00 & 0,7535 & 0,9374 & 1,0392 & 6,00 \\ \text { OT TAM } & 0,8354 & 3,00 & 0,6838 & 0,8353 & 0,9284 & 4,00 \\ \text { OT TQM } & 0,8355 & 4,00 & 0,6858 & 0,8368 & 0,9299 & 5,00 \\ \text { NOT naïve } & 0,9047 & 6,00 & 0,5859 & 0,7448 & 0,8439 & 1,67 \\ \text { NOT média } & 0,8268 & 2,00 & 0,5826 & 0,7451 & 0,8448 & 2,00\end{array}$

\begin{tabular}{|c|c|c|c|c|c|c|}
\hline ZERO & Treino & & & Teste & & \\
\hline Otimização & $\begin{array}{c}\text { a } 1 \text { passo } \\
32 \mathrm{p} .\end{array}$ & $\begin{array}{c}\text { Ranking } \\
\text { treino }\end{array}$ & $T+1$ & $T+3$ & $\mathrm{~T}+6$ & $\begin{array}{c}\text { Ranking } \\
\text { teste }\end{array}$ \\
\hline OT DMA & 1,8025 & 3,50 & 1,1078 & 1,3938 & 1,5285 & 3,50 \\
\hline OT DQM & 1,8025 & 3,50 & 1,1078 & 1,3938 & 1,5285 & 3,50 \\
\hline ОТ TAM & 1,8025 & 3,50 & 1,1078 & 1,3938 & 1,5285 & 3,50 \\
\hline OT TQM & 1,8025 & 3,50 & 1,1078 & 1,3938 & 1,5285 & 3,50 \\
\hline NOT naïve & 1,8025 & 3,50 & 1,1078 & 1,3938 & 1,5285 & 3,50 \\
\hline NOT média & 1,8025 & 3,50 & 1,1078 & 1,3938 & 1,5285 & 3,50 \\
\hline NAIVE & Treino & & & Teste & & \\
\hline Otimização & $\begin{array}{c}\text { a } 1 \text { passo } \\
32 \mathrm{p} .\end{array}$ & $\begin{array}{c}\text { Ranking } \\
\text { treino }\end{array}$ & T & $T$ & & $\begin{array}{c}\text { Ranking } \\
\text { teste }\end{array}$ \\
\hline OT DMA & 1,4726 & 3,50 & 1,0262 & 1,3131 & 1,4438 & 3,50 \\
\hline OT DQM & 1,4726 & 3,50 & 1,0262 & 1,3131 & 1,4438 & 3,50 \\
\hline ОТ TAM & 1,4726 & 3,50 & 1,0262 & 1,3131 & 1,4438 & 3,50 \\
\hline OT TQM & 1,4726 & 3,50 & 1,0262 & 1,3131 & 1,4438 & 3,50 \\
\hline NOT naïve & 1,4726 & 3,50 & 1,0262 & 1,3131 & 1,4438 & 3,50 \\
\hline NOT média & 1,4726 & 3,50 & 1,0262 & 1,3131 & 1,4438 & 3,50 \\
\hline TSB & Treino & & & Teste & & \\
\hline Otimização & $\begin{array}{c}\text { a } 1 \text { passo } \\
32 \mathrm{p} .\end{array}$ & $\begin{array}{c}\text { Ranking } \\
\text { treino }\end{array}$ & $T$ & $T$ & 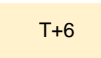 & $\begin{array}{c}\text { Ranking } \\
\text { teste }\end{array}$ \\
\hline OT DMA & 0,8505 & 2,00 & 0,5591 & 0,7612 & 0,8560 & 2,33 \\
\hline OT DQM & 0,7834 & 1,00 & 0,7458 & 0,9440 & 1,0515 & 6,00 \\
\hline OT TAM & 0,8629 & 4,00 & 0,6757 & 0,8367 & 0,9327 & 4,33 \\
\hline OT TQM & 0,8559 & 3,00 & 0,6815 & 0,8383 & 0,9322 & 4,67 \\
\hline NOT naïve & 0,9232 & 6,00 & 0,5613 & 0,7216 & 0,8203 & 2,33 \\
\hline NOT média & 0,9095 & 5,00 & 0,5612 & 0,7216 & 0,8203 & 1,33 \\
\hline
\end{tabular}


TAME TAXA ABSOLUTA MÉDIA ESCALONADA

\begin{tabular}{|c|c|c|c|c|c|c|}
\hline MMS3 & Treino & & & Teste & & \\
\hline $\begin{array}{c}\text { Otimizaçã } \\
0\end{array}$ & $\begin{array}{c}\text { a } 1 \text { passo } \\
32 \mathrm{p} .\end{array}$ & $\begin{array}{l}\text { Ranking } \\
\text { treino }\end{array}$ & $\mathrm{T}+1$ & $T+3$ & $T+6$ & $\begin{array}{c}\text { Ranking } \\
\text { teste }\end{array}$ \\
\hline OT DMA & 11,0338 & 3,50 & 0,4983 & 1,4852 & 2,9347 & 3,50 \\
\hline OT DQM & 11,0338 & 3,50 & 0,4983 & 1,4852 & 2,9347 & 3,50 \\
\hline OT TAM & 11,0338 & 3,50 & 0,4983 & 1,4852 & 2,9347 & 3,50 \\
\hline OT TQM & 11,0338 & 3,50 & 0,4983 & 1,4852 & 2,9347 & 3,50 \\
\hline NOT naïve & 11,0338 & 3,50 & 0,4983 & 1,4852 & 2,9347 & 3,50 \\
\hline NOT média & 11,0338 & 3,50 & 0,4983 & 1,4852 & 2,9347 & 3,50 \\
\hline
\end{tabular}

SBA

\begin{tabular}{ccccccc} 
SBA & Treino & \multicolumn{5}{c}{ Teste } \\
Otimizaçã & a 1 passo & $\begin{array}{c}\text { Ranking } \\
\text { treino }\end{array}$ & $\mathrm{T}+1$ & $\mathrm{~T}+3$ & $\mathrm{~T}+6$ & $\begin{array}{c}\text { Ranking } \\
\text { teste }\end{array}$
\end{tabular}

$\begin{array}{cccccccc}\text { O } & 32 \mathrm{p} . & \text { treino } & & & & & \text { teste } \\ \text { OT DMA } & 9,7320 & 6,00 & 0,3437 & 1,0158 & 1,9893 & 6,00\end{array}$

$\begin{array}{lllllll}\text { OT DQM } & 6,9407 & 5,00 & 0,2789 & 0,8278 & 1,6323 & 5,00\end{array}$

$\begin{array}{llllllll}\text { OT TAM } & 3,1036 & 2,00 & 0,0877 & 0,2799 & 0,6032 & 2,00\end{array}$

$\begin{array}{lllllll}\text { OT TQM } & 3,0308 & 1,00 & 0,0843 & 0,2670 & 0,5794 & 1,00\end{array}$

$\begin{array}{lllllll}\text { NOT naive } \quad 5,3897 & 4,00 & 0,1383 & 0,4112 & 0,8098 & 3,00\end{array}$

$\begin{array}{lllllll}\text { NOT média } & 5,3303 & 3,00 & 0,1394 & 0,4153 & 0,8177 & 4,00\end{array}$ \begin{tabular}{ccccccc} 
CROSTON & Treino & \multicolumn{5}{c}{ Teste } \\
Otimizaçã & $\begin{array}{c}\text { a } 1 \text { passo } \\
0\end{array}$ & $\begin{array}{c}\text { Ranking } \\
\text { treino }\end{array}$ & $\mathrm{T}+1$ & $\mathrm{~T}+3$ & $\mathrm{~T}+6$ & $\begin{array}{c}\text { Ranking } \\
\text { teste }\end{array}$
\end{tabular} $\begin{array}{lllllll}\text { OT DMA } & 9,2890 & 6,00 & 0,3197 & 0,9461 & 1,8514 & 6,00\end{array}$ $\begin{array}{llllllll}\text { OT DQM } & 6,8612 & 5,00 & 0,2677 & 0,7968 & 1,5773 & 5,00\end{array}$ $\begin{array}{lllllll}\text { OT TAM } \quad 3,2067 & 2,00 & 0,0901 & 0,2868 & 0,6218 & 2,00\end{array}$ $\begin{array}{llllllll}\text { OT TQM } & 3,1450 & 1,00 & 0,0814 & 0,2622 & 0,5751 & 1,00\end{array}$ $\begin{array}{lllllll}\text { NOT naïve } & 5,3356 & 4,00 & 0,1385 & 0,4152 & 0,8271 & 3,00\end{array}$ NOT média $\begin{array}{llllll}5,1723 & 3,00 & 0,1395 & 0,4185 & 0,8342 & 4,00\end{array}$

\begin{tabular}{|c|c|c|c|c|c|c|}
\hline SBJ & Treino & & & Teste & & \\
\hline $\begin{array}{c}\text { Otimizaçã } \\
0\end{array}$ & $\begin{array}{c}\text { a } 1 \text { passo } \\
32 \mathrm{p} .\end{array}$ & $\begin{array}{c}\text { Ranking } \\
\text { treino }\end{array}$ & $T+1$ & $T+3$ & $\mathrm{~T}+6$ & $\begin{array}{l}\text { Ranking } \\
\text { teste }\end{array}$ \\
\hline OT DMA & 9,9371 & 6,00 & 0,3522 & 1,0412 & 2,0390 & 6,00 \\
\hline OT DQM & 7,0402 & 5,00 & 0,2808 & 0,8321 & 1,6378 & 5,00 \\
\hline ОТ TAM & 3,1176 & 1,00 & 0,0878 & 0,2792 & 0,5998 & 2,00 \\
\hline От TQM & 3,1216 & 2,00 & 0,0851 & 0,2704 & 0,5882 & 1,00 \\
\hline NOT naïve & 5,4035 & 4,00 & 0,1387 & 0,4121 & 0,8110 & 3,00 \\
\hline NOT média & 5,3495 & 3,00 & 0,1397 & 0,4162 & 0,8190 & 4,00 \\
\hline
\end{tabular}
NOT média $5,3495 \quad 3,00 \quad 0,1397 \quad 0,41620,8190 \quad 4,00$ \begin{tabular}{lllcccc} 
SES & Treino & \multicolumn{3}{c}{ Teste } \\
Otimizaçã a 1 passo & Ranking & $T+1$ & $T+3$ & $T+6$ & Ranking
\end{tabular} $\begin{array}{ccccccc}\circ & 32 \mathrm{p} . & \text { treino } & & & & \\ \text { OT DMA } & 9,1087 & 6,00 & 0,3254 & 0,9651 & 1,8921 & 6,00\end{array}$ $\begin{array}{lllllll}\text { OT DQM } & 6,4847 & 5,00 & 0,2378 & 0,7190 & 1,4419 & 5,00\end{array}$ $\begin{array}{llllllll}\text { OT TAM } & 3,1546 & 1,00 & 0,0366 & 0,1203 & 0,2922 & 1,33\end{array}$ $\begin{array}{lllllll}\text { OT TQM } & 3,1678 & 2,00 & 0,0364 & 0,1214 & 0,2945 & 1,67\end{array}$ NOT naïve $\begin{array}{llllll}5,4887 & 4,00 & 0,1818 & 0,5300 & 1,0180 & 3,00\end{array}$ NOT média $5,1478 \quad 3,00 \quad 0,1856 \quad 0,5414 \quad 1,0434 \quad 4,00$

\begin{tabular}{lclcccc} 
ZERO & Treino & \multicolumn{3}{c}{ Teste } \\
Otimizaçã & a 1 passo & Ranking & $\mathrm{T}+1$ & $\mathrm{~T}+3$ & $\mathrm{~T}+6$ & Ranking
\end{tabular} $\begin{array}{cccccccc}\circ & 32 \mathrm{p} . & \text { treino } & T+1 & T+3 & T+6 & \text { teste } \\ \text { OT DMA } & 33,0354 & 3,50 & 0,9907 & 2,9595 & 5,8682 & 3,50\end{array}$ $\begin{array}{lllllll}\text { OT DMA } & 33,0354 & 3,50 & 0,9907 & 2,9595 & 5,8682 & 3,50 \\ \text { OT DQM } & 33,0354 & 3,50 & 0,9907 & 2,9595 & 5,8682 & 3,50\end{array}$ $\begin{array}{lllllll}\text { OT TAM } & 33,0354 & 3,50 & 0,9907 & 2,9595 & 5,8682 & 3,50\end{array}$ $\begin{array}{llllllll}\text { OT TQM } & 33,0354 & 3,50 & 0,9907 & 2,9595 & 5,8682 & 3,50\end{array}$ $\begin{array}{lllllll}\text { NOT naive } 33,0354 & 3,50 & 0,9907 & 2,9595 & 5,8682 & 3,50\end{array}$ NOT média 33,0354 $3,50 \quad 0,9907 \quad 2,9595 \quad 5,8682 \quad 3,50$

$\begin{array}{ccccccc}\begin{array}{c}\text { NAIVE } \\ \text { Otimizaçã }\end{array} & \begin{array}{c}\text { Treino } \\ \text { a passo } \\ \text { o }\end{array} & \begin{array}{c}\text { Ranking } \\ \text { treino }\end{array} & \mathrm{T}+1 & \begin{array}{c}\text { Teste } \\ \text { t+3 }\end{array} & \mathrm{T}+6 & \begin{array}{c}\text { Ranking } \\ \text { teste }\end{array} \\ \text { OT DMA } & 19,3904 & 3,50 & 0,6851 & 2,0401 & 4,0350 & 3,50 \\ \text { OT DQM } & 19,3904 & 3,50 & 0,6851 & 2,0401 & 4,0350 & 3,50 \\ \text { OT TAM } & 19,3904 & 3,50 & 0,6851 & 2,0401 & 4,0350 & 3,50 \\ \text { OT TQM } & 19,3904 & 3,50 & 0,6851 & 2,0401 & 4,0350 & 3,50 \\ \text { NOT naïve } & 19,3904 & 3,50 & 0,6851 & 2,0401 & 4,0350 & 3,50 \\ \text { NOT média 19,3904 } & 3,50 & 0,6851 & 2,0401 & 4,0350 & 3,50\end{array}$

$\begin{array}{lccccccc}\text { TSB } & \begin{array}{c}\text { Treino } \\ \text { Otimizaçã } \\ \text { a 1 passo } \\ \quad\end{array} & \begin{array}{c}\text { Ranking } \\ \text { treino }\end{array} & \begin{array}{c}\text { Teste } \\ \text { tre }\end{array} & \mathrm{T}+3 & \mathrm{~T}+6 & \begin{array}{c}\text { Ranking } \\ \text { teste }\end{array} \\ \text { OT DMA } & 9,3954 & 6,00 & 0,3398 & 1,0027 & 1,9578 & 6,00 \\ \text { OT DQM } & 6,6459 & 5,00 & 0,2884 & 0,8562 & 1,6809 & 5,00 \\ \text { OT TAM } & 2,9398 & 2,00 & 0,0608 & 0,1935 & 0,4298 & 2,00 \\ \text { OT TQM } & 2,8793 & 1,00 & 0,0557 & 0,1804 & 0,4072 & 1,00 \\ \text { NOT naïve } & 5,8324 & 4,00 & 0,2243 & 0,6544 & 1,2619 & 3,00 \\ \text { NOT média } & 5,8003 & 3,00 & 0,2244 & 0,6545 & 1,2621 & 4,00\end{array}$

TQME TAXA DO QUADRADO DA MÉDIA ESCALONADA

\begin{tabular}{lccccccc} 
MMS3 & $\begin{array}{c}\text { Treino } \\
\text { Otimização }\end{array} \begin{array}{c}\text { 1 passo 32 } \\
\text { p. }\end{array}$ & $\begin{array}{c}\text { Ranking } \\
\text { treino }\end{array}$ & \multicolumn{3}{c}{ Teste } \\
OT DMA & 7,3822 & 3,50 & 0,4277 & 1,2595 & 2,4536 & 3,50 \\
OT DQM & 7,3822 & 3,50 & 0,4277 & 1,2595 & 2,4536 & 3,50 \\
OT TAM & 7,3822 & 3,50 & 0,4277 & 1,2595 & 2,4536 & 3,50 \\
OT TQM & 7,3822 & 3,50 & 0,4277 & 1,2595 & 2,4536 & 3,50 \\
NOT naïv & 7,3822 & 3,50 & 0,4277 & 1,2595 & 2,4536 & 3,50 \\
NOT média & 7,3822 & 3,50 & 0,4277 & 1,2595 & 2,4536 & 3,50
\end{tabular}

\begin{tabular}{|c|c|c|c|c|c|c|}
\hline SBA & Treino & & & Teste & & \\
\hline Otimização & $\begin{array}{c}\text { a } 1 \text { passo } 32 \\
\text { p. }\end{array}$ & $\begin{array}{c}\text { Ranking } \\
\text { treino }\end{array}$ & $T+1$ & $T+3$ & $T+6$ & $\begin{array}{c}\text { Ranking } \\
\text { teste }\end{array}$ \\
\hline OT DMA & 5,6062 & 6,00 & 0,1950 & 0,5690 & 1,0863 & 5,00 \\
\hline OT DQM & 3,0948 & 5,00 & 0,3233 & 0,9591 & 1,8871 & 6,00 \\
\hline OT TAM & 0,9081 & 2,00 & 0,0176 & 0,0577 & 0,1293 & 2,00 \\
\hline OT TQM & 0,8645 & 1,00 & 0,0144 & 0,0476 & 0,1098 & 1,00 \\
\hline NOT naïe & 2,0456 & 4,00 & 0,0307 & 0,0915 & 0,1826 & 3,00 \\
\hline NOT média & 1,9112 & 3,00 & 0,0312 & 0,0929 & 0,1854 & 4,00 \\
\hline
\end{tabular}

\begin{tabular}{|c|c|c|c|c|c|c|}
\hline CROSTON & Treino & & & Teste & & \\
\hline Otimizaç & $\begin{array}{c}\text { a } 1 \text { passo } 32 \\
\text { p. }\end{array}$ & $\begin{array}{l}\text { Ranking } \\
\text { treino }\end{array}$ & $\mathrm{T}+1$ & $T+3$ & $T+6$ & $\begin{array}{c}\text { Ranking } \\
\text { teste }\end{array}$ \\
\hline OT DMA & 5,3006 & 6,00 & 0,1800 & 0,5264 & 1,0059 & 5,00 \\
\hline OT DQM & 3,0644 & 5,00 & 0,3260 & 0,9683 & 1,9093 & 6,00 \\
\hline OT TAM & 0,9692 & 2,00 & 0,0207 & 0,0667 & 0,1494 & 2,00 \\
\hline OT TQM & 0,9523 & 1,00 & 0,0162 & 0,0538 & 0,1244 & 1,00 \\
\hline NOT naïve & 2,1687 & 4,00 & 0,0328 & 0,0989 & 0,2019 & 3,00 \\
\hline NOT média & 1,9328 & 3,00 & 0,0333 & 0,1002 & 0,2048 & 4,00 \\
\hline
\end{tabular}

NOT média $\quad \begin{array}{lllllll}1,9328 & 3,00 & 0,0333 & 0,1002 & 0,2048 & 4,00\end{array}$

\begin{tabular}{|c|c|c|c|c|c|c|}
\hline SBJ & Treino & & & Teste & & \\
\hline Otimização & $\begin{array}{c}\text { a } 1 \text { passo } 32 \\
\text { p. }\end{array}$ & $\begin{array}{c}\text { Ranking } \\
\text { treino }\end{array}$ & $\mathrm{T}+1$ & $T+3$ & $T+6$ & $\begin{array}{c}\text { Ranking } \\
\text { teste }\end{array}$ \\
\hline ОT DMA & 5,8770 & 6,00 & 0,2025 & 0,5913 & 1,1304 & 5,00 \\
\hline OT DQM & 3,1799 & 5,00 & 0,3262 & 0,9668 & 1,9010 & 6,00 \\
\hline ОТ TAM & 0,9247 & 1,00 & 0,0173 & 0,0573 & 0,1297 & 2,00 \\
\hline От TQM & 0,9302 & 2,00 & 0,0167 & 0,0543 & 0,1238 & 1,00 \\
\hline NOT naïve & 2,0444 & 4,00 & 0,0308 & 0,0916 & 0,1825 & 3,00 \\
\hline NOT média & 1,9151 & 3,00 & 0,0312 & 0,0929 & 0,1852 & 4,00 \\
\hline
\end{tabular}

\begin{tabular}{|c|c|c|c|c|c|c|}
\hline SES & Treino & & & Teste & & \\
\hline Otimização & $\begin{array}{c}\text { a } 1 \text { passo } 32 \\
\text { p. }\end{array}$ & $\begin{array}{c}\text { Ranking } \\
\text { treino }\end{array}$ & $\mathrm{T}+1$ & $T+3$ & $T+6$ & $\begin{array}{c}\text { Ranking } \\
\text { teste }\end{array}$ \\
\hline OT DMA & 5,7297 & 6,00 & 0,2070 & 0,6025 & 1,1473 & 5,00 \\
\hline OT DQM & 3,0610 & 5,00 & 0,3366 & 0,9931 & 1,9405 & 6,00 \\
\hline ОТ TAM & 1,4024 & 2,00 & 0,0025 & 0,0091 & 0,0253 & 1,00 \\
\hline OT TQM & 1,4001 & 1,00 & 0,0026 & 0,0096 & 0,0264 & 2,00 \\
\hline NOT naïve & 2,2023 & 4,00 & 0,0560 & 0,1574 & 0,2902 & 3,00 \\
\hline NOT média & 2,0391 & 3,00 & 0,0584 & 0,1645 & 0,3043 & 4,00 \\
\hline
\end{tabular}
\begin{tabular}{lclcccc} 
ZERO & Treino & \multicolumn{3}{c}{ Teste } & \\
Otimização a 1 passo 32 & Ranking & $T+1$ & $T+3$ & $T+6$ & Ranking
\end{tabular} $\begin{array}{lcccccc}\text { Otimização } & \begin{array}{c}\text { 1 passo 32 } \\ \text { p. }\end{array} & \begin{array}{c}\text { Ranking } \\ \text { treino }\end{array} & \mathrm{T}+1 & \mathrm{~T}+3 & \mathrm{~T}+6 & \begin{array}{c}\text { Ranking } \\ \text { teste }\end{array} \\ \text { OT DMA } & 37,4282 & 3,50 & 0,9821 & 2,9253 & 5,7627 & 3,50\end{array}$ $\begin{array}{lllllll}\text { OT DMA } & 37,4282 & 3,50 & 0,9821 & 2,9253 & 5,7627 & 3,50 \\ \text { OT DQM } & 37,4282 & 3,50 & 0,9821 & 2,9253 & 5,7627 & 3,50\end{array}$ $\begin{array}{lllllll}\text { OT TAM } \quad 37,4282 & 3,50 & 0,9821 & 2,9253 & 5,7627 & 3,50\end{array}$ $\begin{array}{lllllll}\text { Oт TQM } & 37,4282 & 3,50 & 0,9821 & 2,9253 & 5,7627 & 3,50\end{array}$ $\begin{array}{lllllll}\text { NOT naive } \quad 37,4282 & 3,50 & 0,9821 & 2,9253 & 5,7627 & 3,50\end{array}$

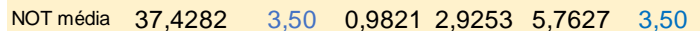

\begin{tabular}{|c|c|c|c|c|c|c|}
\hline NAIVE & Treino & & & Teste & & \\
\hline timização & $\begin{array}{c}\text { a } 1 \text { passo } 32 \\
\text { p. }\end{array}$ & $\begin{array}{c}\text { Ranking } \\
\text { treino }\end{array}$ & $T+1$ & $T+3$ & & $\begin{array}{c}\text { Ranking } \\
\text { teste }\end{array}$ \\
\hline OT DMA & 22,8296 & 3,50 & 0,8950 & 2,6565 & 5,2383 & 3,50 \\
\hline OT DQM & 22,82 & 3,50 & 0,8 & 5 & 3 &, 50 \\
\hline T TAM & 22,8296 & 3,50 & 0,8 & 2,6565 & 5, & 3,50 \\
\hline OT TQM & 22,8296 & 3,50 & 0,8 & 2,6565 & 5,2383 & 3,50 \\
\hline NOT naïve & 22,8296 & 3,50 & 0,8 & 2,6565 & 5,2 & 3,50 \\
\hline VOT média & 22,8296 & 3,50 & 0,8950 & 2,6565 & 5,2383 & 3,50 \\
\hline
\end{tabular}
$\begin{array}{lcccccc}\text { TSB } & \begin{array}{c}\text { Treino } \\ \text { O 1 passo 32 } \\ \text { p. }\end{array} & \begin{array}{c}\text { Ranking } \\ \text { treino }\end{array} & \mathrm{T}+1 & \mathrm{~T}+3 & \mathrm{~T}+6 & \begin{array}{c}\text { Ranking } \\ \text { teste }\end{array}\end{array}$ $\begin{array}{lcccccc} & \text { p. } & \text { treino } & & & & \text { teste } \\ \text { OT DMA } & 5,4571 & 5,00 & 0,2071 & 0,6030 & 1,1474 & 5,00\end{array}$ $\begin{array}{lllllll}\text { OT DQM } & 3,0759 & 4,00 & 0,3523 & 1,0403 & 2,0348 & 6,00\end{array}$ $\begin{array}{lllllll}\text { От тАM } & 144,0439 & 6,00 & 0,0104 & 0,0325 & 0,0716 & 2,00\end{array}$ $\begin{array}{lllllll}\text { OT TQM } & 0,8924 & 1,00 & 0,0079 & 0,0253 & 0,0573 & 1,00\end{array}$ $\begin{array}{lllllll}\text { NOT naive } & 2,4114 & 3,00 & 0,0971 & 0,2754 & 0,5106 & 3,00\end{array}$ $\begin{array}{lllllll}\text { NOT média } & 2,3201 & 2,00 & 0,0972 & 0,2756 & 0,5109 & 4,00\end{array}$ 


\begin{tabular}{|c|c|c|c|c|c|c|}
\hline MMS3 & Treino & & & Teste & & \\
\hline Otimização & $\begin{array}{c}\text { a } 1 \text { passo } 32 \\
\text { p. }\end{array}$ & $\begin{array}{c}\text { Ranking } \\
\text { treino }\end{array}$ & $\mathrm{T}+1$ & $T+3$ & $\mathrm{~T}+6$ & $\begin{array}{c}\text { Ranking } \\
\text { teste }\end{array}$ \\
\hline OT DMA & $-3,1038$ & 6,00 & 0,1817 & 0,6402 & 2,1589 & 6,00 \\
\hline OT DQM & $-3,1038$ & 6,00 & 0,1817 & 0,6402 & 2,1589 & 6,00 \\
\hline ОT TAM & $-3,1038$ & 6,00 & 0,1817 & 0,6402 & 2,1589 & 6,00 \\
\hline OT TQM & $-3,1038$ & 6,00 & 0,1817 & 0,6402 & 2,1589 & 6,00 \\
\hline NOT naïve & $-3,1038$ & 6,00 & 0,1817 & 0,6402 & 2,1589 & 6,00 \\
\hline NOT média & $-3,1038$ & 6,00 & 0,1817 & 0,6402 & 2,1589 & 6,00 \\
\hline
\end{tabular}

\begin{tabular}{|c|c|c|c|c|c|c|}
\hline $3 A$ & Treino & & & Teste & & \\
\hline ção & $\begin{array}{c}\text { a } 1 \text { passo } 32 \\
\text { p. }\end{array}$ & $\begin{array}{c}\text { Ranking } \\
\text { treino }\end{array}$ & $T+1$ & $T+3$ & $T+6$ & \\
\hline T DMA & $-120,2883$ & 6,00 & 0,0841 & 0,0545 & 0,1090 & \\
\hline T DQM & $-41,6348$ & 5,00 & 0,2517 & 1,0603 & & \\
\hline T TAM & $-21,0172$ & 2,00 & 0,3082 & 1,3 & 4 & \\
\hline T TQM & $-15,4721$ & 1,00 & 0,3 & & & \\
\hline DT naïve & $-25,1645$ & 3,00 & 0,2 & 1, & & \\
\hline DT média & $-38,6950$ & 4, & 0 , & 1 , & & \\
\hline ROSTON & Treino & & & Teste & & \\
\hline o & $\begin{array}{c}\text { a } 1 \text { passo } 32 \\
\text { p. }\end{array}$ & $\begin{array}{c}\text { Ranking } \\
\text { treino }\end{array}$ & $T+1$ & $T+3$ & & \\
\hline T DMA & $-96,9476$ & 6,00 & 0 & & & \\
\hline T DQM & $-25,8553$ & 5,00 & 0,2 & & & \\
\hline T TAM & $-17,4427$ & 4,00 & 0,3 & 1, & 08 & \\
\hline T TQM & $-11,8228$ & 2,00 & 0,3249 & 1,4996 & 5,1665 & \\
\hline TT naïve & 0,5514 & 1,00 & 0,3 & & & \\
\hline DT média & -136912 & 300 & 0,3104 & 1,4127 & 4,8625 & \\
\hline
\end{tabular}

\begin{tabular}{|c|c|c|c|c|c|c|}
\hline SBJ & Treino & & & Teste & & \\
\hline Otimização & $\begin{array}{c}\text { a } 1 \text { passo } 32 \\
\text { p. }\end{array}$ & $\begin{array}{c}\text { Ranking } \\
\text { treino }\end{array}$ & $T+1$ & $T+3$ & $T+6$ & $\begin{array}{c}\text { Ranking } \\
\text { teste }\end{array}$ \\
\hline OT DMA & $-124,3908$ & 6,00 & 0,0789 & 0,0236 & 0,0005 & 1,00 \\
\hline OT DQM & $-44,4372$ & 5,00 & 0,2507 & 1,0540 & 3,6072 & 2,00 \\
\hline OT TAM & $-23,3587$ & 2,00 & 0,3094 & 1,4065 & 4,8408 & 5,00 \\
\hline OT TQM & $-16,2428$ & 1,00 & 0,3139 & 1,4336 & 4,9358 & 6,00 \\
\hline NOT naïve & $-26,5180$ & 3,00 & 0,2579 & 1,0974 & 3,7589 & 4,00 \\
\hline NOT média & $-40,0110$ & 4,00 & 0,2576 & 1,0956 & 3,7525 & 3,00 \\
\hline
\end{tabular}

\begin{tabular}{|c|c|c|c|c|c|c|}
\hline SES & Treino & & & Teste & & \\
\hline Otimização & $\begin{array}{c}\text { a } 1 \text { passo } 32 \\
\text { p. }\end{array}$ & $\begin{array}{c}\text { Ranking } \\
\text { treino }\end{array}$ & $T+1$ & $T+3$ & 6 & $\begin{array}{c}\text { Ranking } \\
\text { teste }\end{array}$ \\
\hline OT DMA & $-60,3134$ & 6,00 & 0,1002 & 0,1514 & 0,4479 & 1,00 \\
\hline OT DQM & 3,4694 & 1,00 & 0,2396 & & &, 00 \\
\hline OT TAM & 8,9494 & 4,00 & 0,2927 & 1,3064 & 4,4903 & 5,00 \\
\hline OT TQM & 10,9289 & 5,00 & 0,2960 & 1,3 & 34 & 6,00 \\
\hline NOT naïve & $-8,4 \mathrm{C}$ & 3,00 & 0,24 & & & 3,00 \\
\hline NOT média & $-5,7163$ & 2,00 & 0,2427 & 1,00 & 91 & 4,00 \\
\hline ZERo & Treino & & & & & \\
\hline Otimize & $\begin{array}{c}\text { a } 1 \text { passo } 32 \\
\text { p. }\end{array}$ & $\begin{array}{c}\text { Ranking } \\
\text { treino }\end{array}$ & $T+1$ & & & $\begin{array}{c}\text { Ranking } \\
\text { teste }\end{array}$ \\
\hline OT DMA & $-547,6016$ & 6,00 & $-0,6938$ & $-4,6129$ & $-16,2270$ & 6,00 \\
\hline OT DQM & $-547,6016$ & 6,00 & $-0,6938$ & $-4,6129$ & $-16,2270$ & 6,00 \\
\hline OT TAM & $-547,6016$ & 6,00 & $-0,6938$ & $-4,6129$ & $-16,2270$ & 6,00 \\
\hline OT TQM & $-547,6016$ & 6,00 & $-0,6938$ & $-4,6129$ & $-16,2270$ & 6,00 \\
\hline NOT naïve & $-547,6016$ & 6,00 & $-0,6938$ & $-4,6129$ & $-16,2270$ & 6,00 \\
\hline NOT média & $-547,6016$ & 6,00 & $-0,6938$ & $-4,6129$ & $-16,2270$ & 6,00 \\
\hline
\end{tabular}

\begin{tabular}{|c|c|c|c|c|c|c|}
\hline NAIVE & Treino & & & Teste & & \\
\hline Otimização & $\begin{array}{c}\text { a } 1 \text { passo } 32 \\
\text { p. }\end{array}$ & $\begin{array}{l}\text { Ranking } \\
\text { treino }\end{array}$ & $T+1$ & $T+3$ & $T+6$ & $\begin{array}{c}\text { Ranking } \\
\text { teste }\end{array}$ \\
\hline OT DMA & $-0,3813$ & 6,00 & 0,1634 & 0,5301 & 1,7736 & 6,00 \\
\hline ОT DQM & $-0,3813$ & 6,00 & 0,1634 & 0,5301 & 1,7736 & 6,00 \\
\hline ОТ TAM & $-0,3813$ & 6,00 & 0,1634 & 0,5301 & 1,7736 & 6,00 \\
\hline OT TQM & $-0,3813$ & 6,00 & 0,1634 & 0,5301 & 1,7736 & 6,00 \\
\hline NOT naïve & $-0,3813$ & 6,00 & 0,1634 & 0,5301 & 1,7736 & 6,00 \\
\hline NOT média & $-0,3813$ & 6,00 & 0,1634 & 0,5301 & 1,7736 & 6,00 \\
\hline$S B$ & Treino & & & Teste & & \\
\hline Otimização & $\begin{array}{c}\text { a } 1 \text { passo } 32 \\
\text { p. }\end{array}$ & $\begin{array}{l}\text { Ranking } \\
\text { treino }\end{array}$ & $T+1$ & $T+$ & & $\begin{array}{c}\text { Ranking } \\
\text { teste }\end{array}$ \\
\hline OT DMA & $-101,3376$ & 6,00 & 0,1055 & 0,1833 & 0,5 & 1,00 \\
\hline OT DQM & $-36,5010$ & 5,00 & 0,2483 & 1,0396 & 3,5567 & 4,00 \\
\hline OT TAM & $-24,6908$ & 4,00 & 0,3017 & 1,3602 & 4,6789 & 5,00 \\
\hline OT TQM & $-15,5339$ & 2,00 & 0,3028 & 1,3669 & 4,7021 & 6,00 \\
\hline NOT naïve & $-12,5005$ & 1,00 & 0,2038 & 0,7727 & 2,6224 & 3,00 \\
\hline NOT média & $-20,8907$ & 3,00 & 0,2037 & 0,7724 & 2,6214 & 2,00 \\
\hline
\end{tabular}

\begin{tabular}{|c|c|c|c|c|c|c|}
\hline MMS3 & Treino & & & Teste & & \\
\hline Otimização & $\begin{array}{c}\text { a } 1 \text { passo } 32 \\
\text { p. }\end{array}$ & $\begin{array}{l}\text { Ranking } \\
\text { treino }\end{array}$ & $\mathrm{T}+1$ & $T+3$ & $T+6$ & $\begin{array}{c}\text { Ranking } \\
\text { teste }\end{array}$ \\
\hline OT DMA & 25,5926 & 3,50 & 0,5604 & 2,8298 & 8,9994 & 3,50 \\
\hline OT DQM & 25,5926 & 3,50 & 0,5604 & 2,8298 & 8,9994 & 3,50 \\
\hline ОТ TAM & 25,5926 & 3,50 & 0,5604 & 2,8298 & 8,9994 & 3,50 \\
\hline OT TQM & 25,5926 & 3,50 & 0,5604 & 2,8298 & 8,9994 & 3,50 \\
\hline NOT naïve & 25,5926 & 3,50 & 0,5604 & 2,8298 & 8,9994 & 3,50 \\
\hline NOT média & 25,5926 & 3,50 & 0,5604 & 2,8298 & 8,9994 & 3,50 \\
\hline
\end{tabular}

$\begin{array}{lcccccc}\text { SBA } & \begin{array}{c}\text { Treino } \\ \text { Otimização }\end{array} \begin{array}{c}\text { 1 passo 32 } \\ \text { p. }\end{array} & \begin{array}{c}\text { Ranking } \\ \text { treino }\end{array} & \begin{array}{c}\text { T+1 } \\ \text { Oteste }\end{array} & \mathrm{T}+3 & \mathrm{~T}+6 & \begin{array}{c}\text { Ranking } \\ \text { teste }\end{array} \\ \text { OT DMA } & 123,9482 & 4,00 & 0,5393 & 2,6711 & 8,0561 & 1,00 \\ \text { OT DQM } & 52,5616 & 1,00 & 0,6187 & 3,0132 & 9,1777 & 2,00 \\ \text { OT TAM } & 72,5456 & 3,00 & 0,6838 & 3,3422 & 10,1926 & 5,67 \\ \text { OT TQM } & 71,0003 & 2,00 & 0,6842 & 3,3267 & 10,1677 & 5,33 \\ \text { NOT naïv } & 135,0345 & 6,00 & 0,6458 & 3,0974 & 9,2756 & 3,67 \\ \text { NOT média } & 128,0454 & 5,00 & 0,6438 & 3,0905 & 9,2929 & 3,33\end{array}$

\begin{tabular}{|c|c|c|c|c|c|c|}
\hline ROSTON & Treino & & & Teste & & \\
\hline Otimização & $\begin{array}{c}\text { a } 1 \text { passo } 32 \\
\text { p. }\end{array}$ & $\begin{array}{c}\text { Ranking } \\
\text { treino }\end{array}$ & $\mathrm{T}+1$ & $T+3$ & & $\begin{array}{r}\text { Rank } \\
\text { test }\end{array}$ \\
\hline OT DMA & 109,3143 & 4,00 & 0,5594 & 2,7688 & 8,2857 & 1,00 \\
\hline OT DQM & 52,6267 & 1,00 & 0,6306 & 3,0871 & 9,4489 & 2,0 \\
\hline OT TAM & 72,9597 & 3,00 & 0,6876 & 3,3510 & 10,2455 & 5,0 \\
\hline OT TQM & 70,4120 & 2,00 & 0,6918 & 3,3702 & 10,3686 & 6,0 \\
\hline VOT naïve & 135,1593 & 6,00 & 0,6715 & 3,2078 & 9,6568 & 3,6 \\
\hline NOT média & 126,2225 & 5,00 & 0,6691 & 3,2010 & 9,6682 & 3,3 \\
\hline
\end{tabular}

\begin{tabular}{|c|c|c|c|c|c|c|}
\hline SBJ & Treino & & & Teste & & \\
\hline Otimização & $\begin{array}{c}\text { a } 1 \text { passo } 32 \\
\text { p. }\end{array}$ & $\begin{array}{c}\text { Ranking } \\
\text { treino }\end{array}$ & $\mathrm{T}+1$ & $\mathrm{~T}+3$ & $T+6$ & $\begin{array}{c}\text { Ranking } \\
\text { teste }\end{array}$ \\
\hline OT DMA & 127,8629 & 4,00 & 0,5407 & 2,6502 & 8,0368 & 1,00 \\
\hline OT DQM & 54,3694 & 1,00 & 0,6139 & 2,9675 & 9,0549 & 2,00 \\
\hline ОТ TAM & 73,3848 & 3,00 & 0,6792 & 3,3406 & 10,2030 & 5,00 \\
\hline От TQM & 70,3888 & 2,00 & 0,6835 & 3,3461 & 10,2538 & 6,00 \\
\hline NOT naïve & 135,1054 & 6,00 & 0,6445 & 3,0920 & 9,2568 & 3,67 \\
\hline NOT média & 128,2595 & 5,00 & 0,6425 & 3,0850 & 9,2745 & 3,33 \\
\hline
\end{tabular}

\begin{tabular}{|c|c|c|c|c|c|c|}
\hline SES & Treino & & & Teste & & \\
\hline Otimização & $\begin{array}{c}\text { a } 1 \text { passo } 32 \\
\text { p. }\end{array}$ & $\begin{array}{c}\text { Ranking } \\
\text { treino }\end{array}$ & $T+1$ & $T+3$ & $T+6$ & $\begin{array}{c}\text { Ranking } \\
\text { teste }\end{array}$ \\
\hline DT DMA & 80,7439 & 5,00 & 0,5241 & 6,9951 & 7,5572 & 2,67 \\
\hline DT DQM & 38,9213 & 2,00 & 0,5960 & 2,8199 & 8,3168 & 1,67 \\
\hline ОТ TAM & 54,6030 & 3,00 & 0,6692 & 3,2104 & 9,7342 & 4,67 \\
\hline OT TQM & 54,7477 & 4,00 & 0,6717 & 3,2215 & 9,7654 & 5,67 \\
\hline NOT naïve & 135,4664 & 6,00 & 0,61 & 2,8249 & 8,3523 & 3,33 \\
\hline NOT média & 17,4919 & 1,00 & 0,6104 & 2,8236 & 8,3781 & 3,00 \\
\hline ERO & Treino & & & rese & & \\
\hline Otimização & $\begin{array}{c}\text { a } 1 \text { passo } 32 \\
\text { p. }\end{array}$ & $\begin{array}{c}\text { Ranking } \\
\text { treino }\end{array}$ & $T+$ & $T+3$ & $T+6$ & $\begin{array}{c}\text { Ranking } \\
\text { teste }\end{array}$ \\
\hline OT DMA & 547,6016 & 3,50 & 0,6938 & 4,6129 & 16,2270 & 3,50 \\
\hline OT DQM & 547,6016 & 3,50 & 0,6938 & 4,6129 & 16,2270 & 3,50 \\
\hline OT TAM & 547,6016 & 3,50 & 0,6938 & 4,6129 & 16,2270 & 3,50 \\
\hline OT TQM & 547,6016 & 3,50 & 0,6938 & 4,6129 & 16,2270 & 3,50 \\
\hline NOT naïve & 547,6016 & 3,50 & 0,6938 & 4,6129 & 16,2270 & 3,50 \\
\hline NOT média & 547,6016 & 3,50 & 0,6938 & 4,6129 & 16,2270 & 3,50 \\
\hline
\end{tabular}

$\begin{array}{lcccccc}\text { NAIVE } & \begin{array}{c}\text { Treino } \\ \text { Otimização }\end{array} \begin{array}{c}\text { passo } 32 \\ \text { p. }\end{array} & \begin{array}{c}\text { Ranking } \\ \text { treino }\end{array} & \begin{array}{c}\text { T+1 } \\ \text { Oteste }\end{array} & \mathrm{T}+3 & \mathrm{~T}+6 & \begin{array}{c}\text { Ranking } \\ \text { teste }\end{array} \\ \text { OT DMA } & 19,0340 & 3,50 & 0,6337 & 3,5306 & 11,7720 & 3,50 \\ \text { OT DQM } & 19,0340 & 3,50 & 0,6337 & 3,5306 & 11,7720 & 3,50 \\ \text { OT TAM } & 19,0340 & 3,50 & 0,6337 & 3,5306 & 11,7720 & 3,50 \\ \text { OT TQM } & 19,0340 & 3,50 & 0,6337 & 3,5306 & 11,7720 & 3,50 \\ \text { NOT naïv } & 19,0340 & 3,50 & 0,6337 & 3,5306 & 11,7720 & 3,50 \\ \text { NOT média } & 19,0340 & 3,50 & 0,6337 & 3,5306 & 11,7720 & 3,50\end{array}$

\begin{tabular}{|c|c|c|c|c|c|c|}
\hline TSB & Treino & & & Teste & & \\
\hline Otimização & $\begin{array}{c}\text { a } 1 \text { passo } 32 \\
\text { p. }\end{array}$ & $\begin{array}{l}\text { Ranking } \\
\text { treino }\end{array}$ & $T+1$ & $T+3$ & $T+6$ & $\begin{array}{l}\text { Ranking } \\
\text { teste }\end{array}$ \\
\hline OT DMA & 109,3882 & 4,00 & 0,5366 & 2,6226 & 7,7677 & 1,67 \\
\hline OT DQM & 47,8243 & 1,00 & 0,5943 & 2,8289 & 8,5160 & 4,00 \\
\hline OT TAM & 72,2890 & 3,00 & 0,6692 & 3,2242 & 9,7838 & 5,33 \\
\hline OT TQM & 66,5142 & 2,00 & 0,6705 & 3,2263 & 9,7665 & 5,67 \\
\hline NOT naïve & 132,4047 & 6,00 & 0,5786 & 2,6473 & 7,7212 & 2,67 \\
\hline NOT média & 126,9662 & 5,00 & 0,5786 & 2,6472 & 7,7208 & 1,67 \\
\hline
\end{tabular}




\section{APÊNDICE D - Classificação de erros dos modelos (demanda alto giro).}

\section{OTIMIZAÇÃO POR DMA}

\begin{tabular}{|c|c|c|c|c|c|c|}
\hline DMAE & Treino & & & Teste & & \\
\hline Mod & $\begin{array}{c}\text { a } 1 \text { passo } 32 \\
\text { p. }\end{array}$ & $\begin{array}{c}\text { Ranking } \\
\text { treino }\end{array}$ & $T+1$ & $\mathrm{~T}+3$ & $T+6$ & $\begin{array}{c}\text { Ranking } \\
\text { teste }\end{array}$ \\
\hline MS3 & 0,7068 & 6,00 & 0,5 & 0,6186 & 0,6495 & \\
\hline (1) & 0 &, 00 & & & & 33 \\
\hline ROS & ,6 & ,00 & 0 & u, & 10 & 00 \\
\hline BJ & $\rho_{2}$ & & & & & 3,67 \\
\hline ES & I & 4,00 & 0,5241 & 0,5667 & 0,5 & 1,00 \\
\hline ZERO & 1,0000 & 8,00 & 0,6938 & 0,7638 & 0,7 & 8,00 \\
\hline AIVE & 0,8317 & 7,00 & & 0,6 & 0,7 & 7,00 \\
\hline SB & 0,6022 & 3,00 & 0,5366 & 0,5739 & 0,5997 & 2,00 \\
\hline
\end{tabular}

\begin{tabular}{|c|c|c|c|c|c|c|}
\hline DQME & Treino & & & Teste & & \\
\hline Mod & $\begin{array}{c}\text { a } 1 \text { passo } 32 \\
\text { p. }\end{array}$ & $\begin{array}{l}\text { Ranking } \\
\text { treino }\end{array}$ & $T-$ & $T+3$ & & $\begin{array}{c}\text { Ranking } \\
\text { teste }\end{array}$ \\
\hline MS & 1,0033 & 6,00 & 0 & 0 & 1 & 6,00 \\
\hline$B A$ & 0,8 & 3,00 & & & & \\
\hline ROS & 0,8 & 00 & & 0,7 & & 5,00 \\
\hline BJ & 0,8 & & & 0,7 & & 3,33 \\
\hline SES & 0,8 & & 0,5 & 0,7 & & 1,33 \\
\hline ZERO & 1,8025 & 8,00 & 1,1078 & 1,3938 & 1,5285 & 8,00 \\
\hline NAIVE & 1,4726 & 7,00 & 1,0262 & 1,3131 & & 7,00 \\
\hline SB & 0,8505 & 2,00 & 0,5591 & 0,7612 & 0,8560 & 2,0 \\
\hline
\end{tabular}

\begin{tabular}{|c|c|c|c|c|c|c|}
\hline EMAE & Treino & & & este & & \\
\hline Modelo & $\begin{array}{c}\text { a } 1 \text { passo } 32 \\
\text { p. }\end{array}$ & $\begin{array}{c}\text { Ranking } \\
\text { treino }\end{array}$ & $T+1$ & $T+3$ & $T+6$ & $\begin{array}{c}\text { Ranking } \\
\text { teste }\end{array}$ \\
\hline MMS3 & 0,8531 & 6,00 & 0,6921 & 0,7541 & 0,7956 & 5,67 \\
\hline SBA & & 2,00 & & & & 3 \\
\hline CROSTON & & & & & & 33 \\
\hline SBJ & & & & & & 0 \\
\hline SES & & & & & & 0 \\
\hline EEF & 4 & 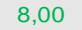 & & 1,0 & 1, &, 00 \\
\hline & P &, 00 & 0 & & & po \\
\hline TSB & 0,7339 & 3,00 & 0,6605 & 0,7002 & 0,7381 & 2,00 \\
\hline
\end{tabular}

\begin{tabular}{|c|c|c|c|c|c|c|}
\hline EEE & Treino & & & Teste & & \\
\hline Mode & $\begin{array}{c}\text { a } 1 \text { passo } 32 \\
\text { p. }\end{array}$ & $\begin{array}{c}\text { Ranking } \\
\text { treino }\end{array}$ & & $T+3$ & & teste \\
\hline MS3 & $-3,1038$ & 1,00 & 0,1817 & 2 & & \\
\hline$B A$ & -12 & & & & & \\
\hline ROS & & & & & & \\
\hline $3 \mathrm{~J}$ & -12 & 6,00 & & & & 0 \\
\hline ES & -60 , & 3,00 & 0,1 & 0,1 & 0,4479 & 3,00 \\
\hline RO & $-547,6016$ & 8,00 & $-0,6938$ & $-4,6129$ & $-16,2270$ & 8,00 \\
\hline AIVE & $-0,3813$ & 2,00 & 0,1634 & 0,5301 & 1,7736 & 6,00 \\
\hline & & & & & 550 & \\
\hline
\end{tabular}

$\begin{array}{lccccrc}\text { TAM } & \begin{array}{c}\text { Treino } \\ \text { M 1 passo 32 }\end{array} & \begin{array}{c}\text { Ranking } \\ \text { treino }\end{array} & \begin{array}{c}\mathrm{T}+1 \\ \text { M. }\end{array} & \begin{array}{c}\text { Teste } \\ \mathrm{T}+3\end{array} & \mathrm{~T}+6 & \begin{array}{c}\text { Ranking } \\ \text { teste }\end{array} \\ \text { MMS3 } & 11,0338 & 6,00 & 0,4983 & 1,4852 & 2,93474 & 6,00 \\ \text { SBA } & 9,7320 & 4,00 & 0,3437 & 1,0158 & 1,98928 & 4,00 \\ \text { CROSTON } & 9,2890 & 2,00 & 0,3197 & 0,9461 & 1,85137 & 1,00 \\ \text { SBJ } & 9,9371 & 5,00 & 0,3522 & 1,0412 & 2,039 & 5,00 \\ \text { SES } & 9,1087 & 1,00 & 0,3254 & 0,9651 & 1,89208 & 2,00 \\ \text { ZERO } & 33,0354 & 8,00 & 0,9907 & 2,9595 & 5,8682 & 8,00 \\ \text { NAIVE } & 19,3904 & 7,00 & 0,6851 & 2,0401 & 4,03501 & 7,00 \\ \text { TSB } & 9,3954 & 3,00 & 0,3398 & 1,0027 & 1,9578 & 3,00\end{array}$

$\begin{array}{lcccccc}\text { TQM } & \begin{array}{c}\text { Treino } \\ \text { a 1 passo 32 } \\ \text { p. }\end{array} & \begin{array}{c}\text { Ranking } \\ \text { treino }\end{array} & \begin{array}{c}\mathrm{T}+1 \\ \text { Modelo }\end{array} & \begin{array}{c}\text { Teste } \\ \mathrm{T}+3\end{array} & \mathrm{~T}+6 & \begin{array}{c}\text { Ranking } \\ \text { teste }\end{array} \\ \text { MMS3 } & 7,3822 & 6,00 & 0,4277 & 1,2595 & 2,4536 & 6,00 \\ \text { SBA } & 5,6062 & 3,00 & 0,1950 & 0,5690 & 1,0863 & 2,00 \\ \text { CROSTON } & 5,3006 & 1,00 & 0,1800 & 0,5264 & 1,0059 & 1,00 \\ \text { SBJ } & 5,8770 & 5,00 & 0,2025 & 0,5913 & 1,1304 & 3,00 \\ \text { SES } & 5,7297 & 4,00 & 0,2070 & 0,6025 & 1,1473 & 4,00 \\ \text { ZERO } & 37,4282 & 8,00 & 0,9821 & 2,9253 & 5,7627 & 8,00 \\ \text { NAIVE } & 22,8296 & 7,00 & 0,8950 & 2,6565 & 5,2383 & 7,00 \\ \text { TSB } & 5,4571 & 2,00 & 0,2071 & 0,6030 & 1,1474 & 5,00\end{array}$

\begin{tabular}{|c|c|c|c|c|c|c|}
\hline PEEEAm & Treino & & & Teste & & \\
\hline Modelo & a 1 passo 32 & $\begin{array}{l}\text { Ranking } \\
\text { treino }\end{array}$ & $T+1$ & $T+3$ & $T+6$ & $\begin{array}{c}\text { Ranking } \\
\text { teste }\end{array}$ \\
\hline MMS3 & 25,5926 & 2,00 & 0,5604 & 2,8298 & 8,9994 & 6,00 \\
\hline SBA & 123,9482 & 6,00 & 0,5393 & 2,6711 & 8,0561 & 3,67 \\
\hline CROSTON & 109,3143 & 4,00 & 0,5594 & 2,7688 & 8,2857 & 5,00 \\
\hline SBJ & 127,8629 & 7,00 & 0,5407 & 2,6502 & 8,0368 & 3,33 \\
\hline SES & 80,7439 & 3,00 & 0,5241 & 2,5441 & 7,5572 & 1,00 \\
\hline ZERO & 547,6016 & 8,00 & 0,6938 & 4,6129 & 16,2270 & 8,00 \\
\hline NAIVE & 19,0340 & 1,00 & 0,6337 & 3,5306 & 11,7720 & 7,00 \\
\hline TSB & 109,3882 & 5.00 & 0,5366 & 2,6226 & 7,7677 & 2,00 \\
\hline
\end{tabular}

\section{OTIMIZAÇÃO POR DQM}

$\begin{array}{lcccccc}\text { DMAE } & \begin{array}{c}\text { Treino } \\ \text { a 1 passo 32 } \\ \text { Modelo }\end{array} & \begin{array}{c}\text { Ranking } \\ \text { p. }\end{array} & \mathrm{T}+1 & \mathrm{~T}+3 & \mathrm{~T}+6 & \begin{array}{c}\text { Ranking } \\ \text { teste }\end{array} \\ \text { MMS3 } & 0,7068 & 6,00 & 0,5604 & 0,6186 & 0,6495 & 1,00 \\ \text { SBA } & 0,6250 & 1,00 & 0,6187 & 0,6546 & 0,6834 & 5,00 \\ \text { CROSTON } & 0,6349 & 5,00 & 0,6306 & 0,6692 & 0,6994 & 6,00 \\ \text { SBJ } & 0,6251 & 2,00 & 0,6139 & 0,6501 & 0,6793 & 4,00 \\ \text { SES } & 0,6314 & 4,00 & 0,5960 & 0,6253 & 0,6515 & 2,33 \\ \text { ZERO } & 1,0000 & 8,00 & 0,6938 & 0,7638 & 0,7857 & 8,00 \\ \text { NAIVE } & 0,8317 & 7,00 & 0,6337 & 0,6914 & 0,7185 & 7,00 \\ \text { TSB } & 0,6271 & 3,00 & 0,5943 & 0,6278 & 0,6573 & 2,67\end{array}$

$\begin{array}{lcccccc}\text { DQME } & \begin{array}{c}\text { Treino } \\ \text { Modelo }\end{array} \begin{array}{c}\text { 1 passo 32 } \\ \text { p. }\end{array} & \begin{array}{c}\text { Ranking } \\ \text { treino }\end{array} & \mathrm{T}+1 & \mathrm{~T}+3 & \mathrm{~T}+6 & \begin{array}{c}\text { Ranking } \\ \text { teste }\end{array} \\ \text { MMS3 } & \mathbf{1 , 0 0 3 3} & 6,00 & 0,6456 & 0,9079 & 1,0281 & 1,00 \\ \text { SBA } & 0,7771 & 2,00 & 0,7634 & 0,9733 & 1,0829 & 5,00 \\ \text { CROSTON } & 0,7849 & 5,00 & 0,7792 & 0,9915 & 1,1006 & 6,00 \\ \text { SBJ } & 0,7808 & 3,00 & 0,7600 & 0,9675 & 1,0768 & 4,00 \\ \text { SES } & 0,7707 & 1,00 & 0,7535 & 0,9374 & 1,0392 & 2,33 \\ \text { ZERO } & 1,8025 & 8,00 & 1,1078 & 1,3938 & 1,5285 & 8,00 \\ \text { NAIVE } & 1,4726 & 7,00 & 1,0262 & 1,3131 & 1,4438 & 7,00 \\ \text { TSB } & 0,7834 & 4,00 & 0,7458 & 0,9440 & 1,0515 & 2,67\end{array}$

\begin{tabular}{lcccccc} 
EMAE & \multicolumn{2}{c}{ Treino } & \multicolumn{5}{c}{ Teste } \\
Modelo & $\begin{array}{c}\text { a } 1 \text { passo } 32 \\
\text { p. }\end{array}$ & $\begin{array}{c}\text { Ranking } \\
\text { treino }\end{array}$ & $\mathrm{T}+1$ & $\mathrm{~T}+3$ & $\mathrm{~T}+6$ & $\begin{array}{c}\text { Ranking } \\
\text { teste }\end{array}$ \\
MMS3 & 0,8531 & 6,00 & 0,6921 & 0,7541 & 0,7956 & 1,00 \\
SBA & 0,7601 & 1,00 & 0,7562 & 0,7933 & 0,8344 & 5,00 \\
CROSTON & 0,7731 & 5,00 & 0,7740 & 0,8130 & 0,8552 & 6,00 \\
SBJ & 0,7605 & 2,00 & 0,7513 & 0,7888 & 0,8300 & 4,00 \\
SES & 0,7655 & 4,00 & 0,7329 & 0,7599 & 0,7975 & 2,33 \\
ZERO & 1,3204 & 8,00 & 0,9303 & 1,0041 & 1,0379 & 8,00 \\
NAIVE & 1,0000 & 7,00 & 0,7899 & 0,8495 & 0,8871 & 7,00 \\
TSB & 0,7616 & 3,00 & 0,7272 & 0,7605 & 0,8032 & 2,67
\end{tabular}

\begin{tabular}{lcccccc} 
PEEE & \multicolumn{2}{c}{ Treino } & \multicolumn{5}{c}{ Teste } \\
Modelo & $\begin{array}{c}\text { a 1 passo 32 } \\
\text { p. }\end{array}$ & $\begin{array}{c}\text { Ranking } \\
\text { treino }\end{array}$ & $\mathrm{T}+1$ & $\mathrm{~T}+3$ & $\mathrm{~T}+6$ & $\begin{array}{c}\text { Ranking } \\
\text { teste }\end{array}$ \\
MMS3 & $-3,1038$ & 2,00 & 0,1817 & 0,6402 & 2,1589 & 2,00 \\
SBA & $-41,6348$ & 6,00 & 0,2517 & 1,0603 & 3,6290 & 6,00 \\
CROSTON & $-25,8553$ & 4,00 & 0,2961 & 1,3267 & 4,5615 & 7,00 \\
SBJ & $-44,4372$ & 7,00 & 0,2507 & 1,0540 & 3,6072 & 5,00 \\
SES & 3,4694 & 3,00 & 0,2396 & 0,9878 & 3,3755 & 3,00 \\
ZERO & $-547,6016$ & 8,00 & $-0,6938$ & $-4,6129$ & $-16,2270$ & 8,00 \\
NAIVE & $-0,3813$ & 1,00 & 0,1634 & 0,5301 & 1,7736 & 1,00 \\
TSB & $-36,5010$ & 5,00 & 0,2483 & 1,0396 & 3,5567 & 4,00
\end{tabular}

\begin{tabular}{|c|c|c|c|c|c|c|}
\hline TAM & Treino & & & Teste & & \\
\hline Modelo & $\begin{array}{c}\text { a } 1 \text { passo } 32 \\
\text { p. }\end{array}$ & $\begin{array}{l}\text { Ranking } \\
\text { treino }\end{array}$ & $T+1$ & $T+3$ & $T+6$ & $\begin{array}{c}\text { Ranking } \\
\text { teste }\end{array}$ \\
\hline MMS3 & 11,0338 & 6,00 & 0,4983 & 1,4852 & 2,93474 & 6,00 \\
\hline SBA & 6,9407 & 4,00 & 0,2789 & 0,8 & 27 & 3,00 \\
\hline CROSTON & 6,8612 &, 00 & 0,2 & 0,7 & 34 & 2,00 \\
\hline SBJ & 7,0402 & 5,00 & 0,2 & 0,8 & 76 & 4,00 \\
\hline SES & 6,4847 & 1,00 & 0,2378 & 0,7190 & 1,44189 & 1,00 \\
\hline ZERO & 33,0354 & 8,00 & 0,9907 & 2,9595 & 5,8682 & 8,00 \\
\hline NAIVE & 19,3904 & 7,00 & 0,6851 & 2,0401 & 4,03501 & 7,00 \\
\hline TSB & 6,6459 & 2,00 & 0,2884 & 0,8562 & 1,68092 & 5,00 \\
\hline
\end{tabular}

$\begin{array}{lcccccc}\text { TQM } & \begin{array}{c}\text { Treino } \\ \text { a 1 passo 32 } \\ \text { Modelo }\end{array} & \begin{array}{c}\text { Ranking } \\ \text { treino }\end{array} & \begin{array}{c}\mathrm{T}+1 \\ \mathrm{p}+\mathrm{T}+3\end{array} & \mathrm{~T}+3+6 & \begin{array}{c}\text { Ranking } \\ \text { teste }\end{array} \\ \text { MMS3 } & 7,3822 & 6,00 & 0,4277 & 1,2595 & 2,4536 & 6,00 \\ \text { SBA } & 3,0948 & 4,00 & 0,3233 & 0,9591 & 1,8871 & 1,00 \\ \text { CROSTON } & 3,0644 & 2,00 & 0,3260 & 0,9683 & 1,9093 & 2,67 \\ \text { SBJ } & 3,1799 & 5,00 & 0,3262 & 0,9668 & 1,9010 & 2,33 \\ \text { SES } & 3,0610 & 1,00 & 0,3366 & 0,9931 & 1,9405 & 4,00 \\ \text { ZERO } & 37,4282 & 8,00 & 0,9821 & 2,9253 & 5,7627 & 8,00 \\ \text { NAIVE } & 22,8296 & 7,00 & 0,8950 & 2,6565 & 5,2383 & 7,00 \\ \text { TSB } & 3,0759 & 3,00 & 0,3523 & 1,0403 & 2,0348 & 5,00\end{array}$

\begin{tabular}{lcccccc} 
PEEEAm & \multicolumn{2}{c}{ Treino } & \multicolumn{5}{c}{ Teste } \\
Modelo & $\begin{array}{c}\text { a passo 32 } \\
\text { p. }\end{array}$ & $\begin{array}{c}\text { Ranking } \\
\text { treino }\end{array}$ & $\begin{array}{c}\mathrm{T}+1 \\
\mathrm{~T}+3\end{array}$ & $\mathrm{~T}+6$ & $\begin{array}{c}\text { Ranking } \\
\text { teste }\end{array}$ \\
MMS3 & 25,5926 & 2,00 & 0,5604 & 2,8298 & 8,9994 & 2,33 \\
SBA & 52,5616 & 5,00 & 0,6187 & 3,0132 & 9,1777 & 5,00 \\
CROSTON & 52,6267 & 6,00 & 0,6306 & 3,0871 & 9,4489 & 6,00 \\
SBJ & 54,3694 & 7,00 & 0,6139 & 2,9675 & 9,0549 & 4,00 \\
SES & 38,9213 & 3,00 & 0,5960 & 2,8199 & 8,3168 & 1,67 \\
ZERO & 547,6016 & 8,00 & 0,6938 & 4,6129 & 16,2270 & 8,00 \\
NAIVE & 19,0340 & 1,00 & 0,6337 & 3,5306 & 11,7720 & 7,00 \\
TSB & 47,8243 & 4,00 & 0,5943 & 2,8289 & 8,5160 & 2,00
\end{tabular}




\section{OTIMIZAÇÃO POR TAM}

\begin{tabular}{|c|c|c|c|c|c|c|}
\hline DMAE & Treino & & & Teste & & \\
\hline Modelo & a 1 passo $32 p$ & $\begin{array}{c}\text { Ranking } \\
\text { treino }\end{array}$ & $T+1$ & $T+3$ & & $\begin{array}{c}\text { Ranking } \\
\text { teste }\end{array}$ \\
\hline MMS3 & 0,7068 & 6,00 & 0,5604 & 0,6186 & 0,6495 & 1,00 \\
\hline SBA & 0,6794 & 3,00 & 0,6838 & 0,7102 & 0,7355 & 5,67 \\
\hline CROSTON & 0,6817 & 5,00 & 0,6876 & 0,7123 & 0,7408 & 7,00 \\
\hline SBJ & 0,6807 & 4,00 & 0,6792 & 0,7091 & 0,7364 & 5,33 \\
\hline SES & 0,6722 & 1,00 & 0,6692 & 0,6890 & 0,7168 & 2,33 \\
\hline ZERO & 1,0000 & 8,00 & 0,6938 & 0,7638 & 0,7857 & 8,00 \\
\hline NAIVE & 0,8317 & 7,00 & 0,6337 & 0,6914 & 0,7185 & 3,00 \\
\hline
\end{tabular}

\begin{tabular}{lcccccc} 
DQME & \multicolumn{1}{c}{ Treino } \\
Modelo & a 1 passo $32 \mathrm{p}$. & $\begin{array}{c}\text { Ranking } \\
\text { treino }\end{array}$ & $\mathrm{T}+1$ & $\mathrm{~T}+3$ & $\mathrm{~T}+6$ & $\begin{array}{c}\text { Ranking } \\
\text { teste }\end{array}$ \\
MMS3 & 1,0033 & 6,00 & 0,6456 & 0,9079 & 1,0281 & 4,33 \\
SBA & 0,8662 & 3,00 & 0,7173 & 0,8810 & 0,9731 & 3,67 \\
CROSTON & 0,8694 & 4,00 & 0,7184 & 0,8823 & 0,9814 & 5,00 \\
SBJ & 0,8702 & 5,00 & 0,7157 & 0,8830 & 0,9767 & 4,33 \\
SES & 0,8354 & 1,00 & 0,6838 & 0,8353 & 0,9284 & 1,67 \\
ZERO & 1,8025 & 8,00 & 1,1078 & 1,3938 & 1,5285 & 8,00 \\
NAIVE & 1,4726 & 7,00 & 1,0262 & 1,3131 & 1,4438 & 7,00 \\
TSB & 0,8629 & 2,00 & 0,6757 & 0,8367 & 0,9327 & 2,00
\end{tabular}

$\begin{array}{lcccccc}\text { EMAE } & \begin{array}{c}\text { Treino } \\ \text { Modelo }\end{array} & \begin{array}{c}\text { 1 passo } 32 \mathrm{p} . \\ \text { Ranking } \\ \text { treino }\end{array} & \mathrm{T}+1 & \mathrm{~T}+3 & \mathrm{~T}+6 & \begin{array}{c}\text { Ranking } \\ \text { teste }\end{array} \\ \text { MMS3 } & 0,8531 & 6,00 & 0,6921 & 0,7541 & 0,7956 & 1,00 \\ \text { SBA } & 0,8262 & 3,00 & 0,8396 & 0,8617 & 0,8985 & 5,67 \\ \text { CROSTON } & 0,8295 & 5,00 & 0,8458 & 0,8651 & 0,9068 & 7,00 \\ \text { SBJ } & 0,8277 & 4,00 & 0,8329 & 0,8591 & 0,8991 & 5,33 \\ \text { SES } & 0,8166 & 1,00 & 0,8271 & 0,8389 & 0,8804 & 2,67 \\ \text { ZERO } & 1,3204 & 8,00 & 0,9303 & 1,0041 & 1,0379 & 8,00 \\ \text { NAIVE } & 1,0000 & 7,00 & 0,7899 & 0,8495 & 0,8871 & 3,33\end{array}$

$\begin{array}{lllllll}\text { TSB } & 0,8237 & 2,00 & 0,8244 & 0,8415 & 0,8805 & 3,00\end{array}$

\begin{tabular}{lcccccc} 
PEEE & \multicolumn{1}{c}{ Treino } & \multicolumn{7}{c}{ Teste } \\
Modelo & a 1 passo 32 p. & $\begin{array}{c}\text { Ranking } \\
\text { treino }\end{array}$ & $\mathrm{T}+1$ & $\mathrm{~T}+3$ & $\mathrm{~T}+6$ & $\begin{array}{c}\text { Ranking } \\
\text { teste }\end{array}$ \\
MMS3 & $-3,1038$ & 2,00 & 0,1817 & 0,6402 & 2,1589 & 2,00 \\
SBA & $-21,0172$ & 5,00 & 0,3082 & 1,3992 & 4,8153 & 5,00 \\
CROSTON & $-17,4427$ & 4,00 & 0,3175 & 1,4551 & 5,0108 & 7,00 \\
SBJ & $-23,3587$ & 6,00 & 0,3094 & 1,4065 & 4,8408 & 6,00 \\
SES & 8,9494 & 3,00 & 0,2927 & 1,3064 & 4,4903 & 3,00 \\
ZERO & $-547,6016$ & 8,00 & $-0,6938$ & $-4,6129$ & $-16,2270$ & 8,00 \\
NAIVE & $-0,3813$ & 1,00 & 0,1634 & 0,5301 & 1,7736 & 1,00 \\
TSB & $-24,6908$ & 7,00 & 0,3017 & 1,3602 & 4,6789 & 4,00
\end{tabular}

\begin{tabular}{lcccccc} 
TAM & \multicolumn{1}{c}{ Treino } & \multicolumn{7}{c}{ Teste } \\
Modelo & $\begin{array}{c}\text { 1 passo 32 p. } \\
\text { Ranking } \\
\text { treino }\end{array}$ & $\mathrm{T}+1$ & $\mathrm{~T}+3$ & $\mathrm{~T}+6$ & $\begin{array}{c}\text { Ranking } \\
\text { teste }\end{array}$ \\
MMS3 & 11,0338 & 6,00 & 0,4983 & 1,4852 & 2,93474 & 6,00 \\
SBA & 3,1036 & 2,00 & 0,0877 & 0,2799 & 0,60321 & 3,67 \\
CROSTON & 3,2067 & 5,00 & 0,0901 & 0,2868 & 0,62178 & 5,00 \\
SBJ & 3,1176 & 3,00 & 0,0878 & 0,2792 & 0,59979 & 3,33 \\
SES & 3,1546 & 4,00 & 0,0366 & 0,1203 & 0,29223 & 1,00 \\
ZERO & 33,0354 & 8,00 & 0,9907 & 2,9595 & 5,8682 & 8,00 \\
NAIVE & 19,3904 & 7,00 & 0,6851 & 2,0401 & 4,03501 & 7,00 \\
TSB & 2,9398 & 1,00 & 0,0608 & 0,1935 & 0,42977 & 2,00
\end{tabular}

\begin{tabular}{|c|c|c|c|c|c|c|}
\hline TQM & Treino & & & Teste & & \\
\hline Modelo & a 1 passo $32 \mathrm{p}$. & $\begin{array}{c}\text { Ranking } \\
\text { treino }\end{array}$ & & $\mathrm{T}+3$ & & $\begin{array}{c}\text { Ranking } \\
\text { teste }\end{array}$ \\
\hline MMS3 & 7,3822 & 6,00 & 0,4277 & 1,2595 & 2,4536 & 6,00 \\
\hline SBA & 0,9081 & 2,00 & 0,0176 & 0,0577 & 0,1293 & 3,67 \\
\hline CROSTON & 0,9692 & 4,00 & 0,0207 & 0,0667 & 0,1494 & 5,00 \\
\hline SBJ & 0,9247 & 3,00 & 0,0173 & 0,0573 & 0,1297 & 3,33 \\
\hline SES & 1,4024 & 5,00 & 0,0025 & 0,0091 & 0,0253 & 1,00 \\
\hline ZERO & 37,4282 & 8,00 & 0,9821 & 2,9253 & 5,7627 & 8,00 \\
\hline NAIVE & 22,8296 & 7,00 & 0,8950 & 2,6565 & 5,2383 & 7,00 \\
\hline TSB & 0,9033 & 1,00 & 0,0104 & 0,0325 & 0,0716 & 2,00 \\
\hline
\end{tabular}

\begin{tabular}{|c|c|c|c|c|c|c|}
\hline PEEEAm & Treino & & & Teste & & \\
\hline Modelo & a 1 passo $32 p$. & $\begin{array}{c}\text { Ranking } \\
\text { treino }\end{array}$ & $T+1$ & $\mathrm{~T}+3$ & & $\begin{array}{c}\text { Ranking } \\
\text { teste }\end{array}$ \\
\hline MMS3 & 25,5926 & 2,00 & 0,5604 & 2,8298 & 8,9994 & 1,00 \\
\hline SBA & 72,5456 & 5,00 & 0,6838 & 3,3422 & 10,1926 & 5,00 \\
\hline CROSTON & 72,9597 & 6,00 & 0,6876 & 3,3510 & 10,2455 & 6,33 \\
\hline SBJ & 73,3848 & 7,00 & 0,6792 & 3,3406 & 10,2030 & 4,67 \\
\hline SES & 54,6030 & 3,00 & 0,6692 & 3,2104 & 9,7342 & 2,33 \\
\hline ZERO & 547,6016 & 8,00 & 0,6938 & 4,6129 & 16,2270 & 8,00 \\
\hline NAIVE & 19,0340 & 1,00 & 0,6337 & 3,5306 & 11,7720 & 5,33 \\
\hline TSB & 72,2890 & 4,00 & 0,6692 & 3,2242 & 9,7838 & 3,33 \\
\hline
\end{tabular}

\section{OTIMIZAÇÃO POR TQM}

\begin{tabular}{lcccccc} 
DMAE & \multicolumn{1}{c}{ Treino } & \multicolumn{5}{c}{ Teste } \\
Modelo & a 1 passo 32 p. $\begin{array}{c}\text { Ranking } \\
\text { treino }\end{array}$ & $\mathrm{T}+1$ & $\mathrm{~T}+3$ & $\mathrm{~T}+6$ & $\begin{array}{c}\text { Ranking } \\
\text { teste }\end{array}$ \\
MMS3 & 0,7068 & 6,00 & 0,5604 & 0,6186 & 0,6495 & 1,00 \\
SBA & 0,6777 & 3,00 & 0,6842 & 0,7085 & 0,7364 & 5,33 \\
CROSTON & 0,6808 & 5,00 & 0,6918 & 0,7157 & 0,7426 & 7,00 \\
SBJ & 0,6781 & 4,00 & 0,6835 & 0,7099 & 0,7380 & 5,67 \\
SES & 0,6730 & 1,00 & 0,6717 & 0,6909 & 0,7183 & 3,00 \\
ZERO & 1,0000 & 8,00 & 0,6938 & 0,7638 & 0,7857 & 8,00 \\
NAIVE & 0,8317 & 7,00 & 0,6337 & 0,6914 & 0,7185 & 3,33 \\
TSB & 0,6760 & 2,00 & 0,6705 & 0,6911 & 0,7179 &
\end{tabular}

$\begin{array}{lllllll}\text { NAIVE } & 0,6760 & 2,00 & 0,6705 & 0,6911 & 0,7179 & 2,67\end{array}$

$\begin{array}{lcccccc}\text { DQME } & \begin{array}{c}\text { Treino } \\ \text { Modelo }\end{array} & \begin{array}{c}\text { 1 passo 32 p. } \\ \text { Ranking } \\ \text { treino }\end{array} & \mathrm{T}+1 & \mathrm{~T}+3 & \mathrm{~T}+6 & \begin{array}{c}\text { Ranking } \\ \text { teste }\end{array} \\ \text { MMS3 } & 1,0033 & 6,00 & 0,6456 & 0,9079 & 1,0281 & 4,33 \\ \text { SBA } & 0,8580 & 3,00 & 0,7109 & 0,8741 & 0,9709 & 3,33 \\ \text { CROSTON } & 0,8634 & 5,00 & 0,7318 & 0,8878 & 0,9838 & 5,33 \\ \text { SBJ } & 0,8588 & 4,00 & 0,7157 & 0,8760 & 0,9754 & 4,33 \\ \text { SES } & 0,8355 & 1,00 & 0,6858 & 0,8368 & 0,9299 & 1,67 \\ \text { ZERO } & 1,8025 & 8,00 & 1,1078 & 1,3938 & 1,5285 & 8,00 \\ \text { NAIVE } & 1,4726 & 7,00 & 1,0262 & 1,3131 & 1,4438 & 7,00 \\ \text { TSB } & 0,8559 & 2,00 & 0,6815 & 0,8383 & 0,9322 & 2,00\end{array}$

\begin{tabular}{lcccccc} 
EMAE & \multicolumn{1}{c}{ Treino } & \multicolumn{5}{c}{ Teste } \\
Modelo & a 1 passo 32 p. & $\begin{array}{c}\text { Ranking } \\
\text { treino }\end{array}$ & $\mathrm{T}+1$ & $\mathrm{~T}+3$ & $\mathrm{~T}+6$ & $\begin{array}{c}\text { Ranking } \\
\text { teste }\end{array}$ \\
MMS3 & 0,8531 & 6,00 & 0,6921 & 0,7541 & 0,7956 & 1,00 \\
SBA & 0,8238 & 3,00 & 0,8381 & 0,8578 & 0,8981 & 5,00 \\
CROSTON & 0,8284 & 5,00 & 0,8505 & 0,8693 & 0,9094 & 7,00 \\
SBJ & 0,8246 & 4,00 & 0,8393 & 0,8617 & 0,9018 & 6,00 \\
SES & 0,8175 & 1,00 & 0,8301 & 0,8411 & 0,8822 & 3,33 \\
ZERO & 1,3204 & 8,00 & 0,9303 & 1,0041 & 1,0379 & 8,00 \\
NAIVE & 1,0000 & 7,00 & 0,7899 & 0,8495 & 0,8871 & 3,33 \\
TSB & 0,8214 & 2,00 & 0,8255 & 0,8395 & 0,8796 & 233
\end{tabular}

\begin{tabular}{lcccccc} 
PEEE & \multicolumn{1}{c}{ Treino } & \multicolumn{5}{c}{ Teste } \\
Modelo & a 1 passo 32 p. & $\begin{array}{c}\text { Ranking } \\
\text { treino }\end{array}$ & $\mathrm{T}+1$ & $\mathrm{~T}+3$ & $\mathrm{~T}+6$ & $\begin{array}{c}\text { Ranking } \\
\text { teste }\end{array}$ \\
MMS3 & $-3,1038$ & 2,00 & 0,1817 & 0,6402 & 2,1589 & 2,00 \\
SBA & $-15,4721$ & 5,00 & 0,3137 & 1,4325 & 4,9318 & 5,00 \\
CROSTON & $-11,8228$ & 4,00 & 0,3249 & 1,4996 & 5,1665 & 7,00 \\
SBJ & $-16,2428$ & 7,00 & 0,3139 & 1,4336 & 4,9358 & 6,00 \\
SES & 10,9289 & 3,00 & 0,2960 & 1,3258 & 4,5584 & 3,00 \\
ZERO & $-547,6016$ & 8,00 & $-0,6938$ & $-4,6129$ & $-16,2270$ & 8,00 \\
NAIVE & $-0,3813$ & 1,00 & 0,1634 & 0,5301 & 1,7736 & 1,00 \\
TSB & $-15,5339$ & 6,00 & 0,3028 & 1,3669 & 4,7021 & 4,00
\end{tabular}

\begin{tabular}{lccccrc} 
TAM & $\begin{array}{c}\text { Treino } \\
\text { Modelo }\end{array}$ & $\begin{array}{c}\text { 1 passo 32 p. } \\
\text { Ranking } \\
\text { treino }\end{array}$ & $\mathrm{T}+1$ & $\mathrm{~T}+3$ & \multicolumn{1}{c}{$\mathrm{T}+6$} & $\begin{array}{c}\text { Ranking } \\
\text { teste }\end{array}$ \\
MMS3 & 11,0338 & 6,00 & 0,4983 & 1,4852 & 2,93474 & 6,00 \\
SBA & 3,0308 & 2,00 & 0,0843 & 0,2670 & 0,57941 & 4,00 \\
CROSTON & 3,1450 & 4,00 & 0,0814 & 0,2622 & 0,5751 & 3,00 \\
SBJ & 3,1216 & 3,00 & 0,0851 & 0,2704 & 0,58821 & 5,00 \\
SES & 3,1678 & 5,00 & 0,0364 & 0,1214 & 0,29454 & 1,00 \\
ZERO & 33,0354 & 8,00 & 0,9907 & 2,9595 & 5,8682 & 8,00 \\
NAIVE & 19,3904 & 7,00 & 0,6851 & 2,0401 & 4,03501 & 7,00 \\
TSB & 2,8793 & 1,00 & 0,0557 & 0,1804 & 0,40721 & 2,00
\end{tabular}

\begin{tabular}{lcccccc} 
TQM & \multicolumn{1}{c}{ Treino } & \multicolumn{5}{c}{ Teste } \\
Modelo & a 1 passo 32 p. & $\begin{array}{c}\text { Ranking } \\
\text { treino }\end{array}$ & $\mathrm{T}+1$ & $\mathrm{~T}+3$ & $\mathrm{~T}+6$ & $\begin{array}{c}\text { Ranking } \\
\text { teste }\end{array}$ \\
MMS3 & 7,3822 & 6,00 & 0,4277 & 1,2595 & 2,4536 & 6,00 \\
SBA & 0,8645 & 1,00 & 0,0144 & 0,0476 & 0,1098 & 3,00 \\
CROSTON & 0,9523 & 4,00 & 0,0162 & 0,0538 & 0,1244 & 4,33 \\
SBJ & 0,9302 & 3,00 & 0,0167 & 0,0543 & 0,1238 & 4,67 \\
SES & 1,4001 & 5,00 & 0,0026 & 0,0096 & 0,0264 & 1,00 \\
ZERO & 37,4282 & 8,00 & 0,9821 & 2,9253 & 5,7627 & 8,00 \\
NAIVE & 22,8296 & 7,00 & 0,8950 & 2,6565 & 5,2383 & 7,00 \\
TSB & 0,8924 & 2,00 & 0,0079 & 0,0253 & 0,0573 & 2,00
\end{tabular}

\begin{tabular}{lcccccc} 
PEEEAm & \multicolumn{2}{c}{ Treino } & \multicolumn{5}{c}{ Teste } \\
Modelo & a 1 passo 32 p. $\begin{array}{c}\text { Ranking } \\
\text { treino }\end{array}$ & $\mathrm{T}+1$ & $\mathrm{~T}+3$ & $\mathrm{~T}+6$ & $\begin{array}{c}\text { Ranking } \\
\text { teste }\end{array}$ \\
MMS3 & 25,5926 & 2,00 & 0,5604 & 2,8298 & 8,9994 & 1,00 \\
SBA & 71,0003 & 7,00 & 0,6842 & 3,3267 & 10,1677 & 4,67 \\
CROSTON & 70,4120 & 6,00 & 0,6918 & 3,3702 & 10,3686 & 6,33 \\
SBJ & 70,3888 & 5,00 & 0,6835 & 3,3461 & 10,2538 & 5,00 \\
SES & 54,7477 & 3,00 & 0,6717 & 3,2215 & 9,7654 & 2,67 \\
ZERO & 547,6016 & 8,00 & 0,6938 & 4,6129 & 16,2270 & 8,00 \\
NAIVE & 19,0340 & 1,00 & 0,6337 & 3,5306 & 11,7720 & 5,33 \\
TSB & 66,5142 & 4,00 & 0,6705 & 3,2263 & 9,7665 & 3,00
\end{tabular}




\section{NÃO OTIMIZADO (INÍCIO Pt = NAIVE)}

\begin{tabular}{lcccccc} 
DMAE & \multicolumn{1}{c}{ Treino } & \multicolumn{7}{c}{ Teste } \\
Modelo & $\begin{array}{c}\text { 1 passo } 32 \\
\text { p. }\end{array}$ & $\begin{array}{c}\text { Ranking } \\
\text { treino }\end{array}$ & $\mathrm{T}+1$ & $\mathrm{~T}+3$ & $\mathrm{~T}+6$ & $\begin{array}{c}\text { Ranking } \\
\text { teste }\end{array}$ \\
MMS3 & 0,7068 & 5,00 & 0,5604 & 0,6186 & 0,6495 & 1,67 \\
SBA & 0,7001 & 4,00 & 0,6458 & 0,6711 & 0,6995 & 5,33 \\
CROSTON & 0,7123 & 6,00 & 0,6715 & 0,6922 & 0,7195 & 7,00 \\
SBJ & 0,6995 & 3,00 & 0,6445 & 0,6700 & 0,6985 & 4,33 \\
SES & 0,6779 & 1,00 & 0,6106 & 0,6287 & 0,6571 & 3,00 \\
ZERO & 1,0000 & 8,00 & 0,6938 & 0,7638 & 0,7857 & 8,00 \\
NAIVE & 0,8317 & 7,00 & 0,6337 & 0,6914 & 0,7185 & 5,33 \\
TSB & 0,6915 & 2,00 & 0,5786 & 0,5980 & 0,6265 & 1,33
\end{tabular}

\begin{tabular}{lcccccc} 
DQME & \multicolumn{2}{c}{ Treino } & \multicolumn{5}{c}{ Teste } \\
Modelo & $\begin{array}{c}\text { a 1 passo 32 } \\
\text { p. }\end{array}$ & $\begin{array}{c}\text { Ranking } \\
\text { treino }\end{array}$ & $\mathrm{T}+1$ & $\mathrm{~T}+3$ & $\mathrm{~T}+6$ & $\begin{array}{c}\text { Ranking } \\
\text { teste }\end{array}$ \\
MMS3 & 1,0033 & 6,00 & 0,6456 & 0,9079 & 1,0281 & 5,00 \\
SBA & 0,9404 & 4,00 & 0,6646 & 0,8272 & 0,9243 & 4,33 \\
CROSTON & 0,9559 & 5,00 & 0,6934 & 0,8495 & 0,9447 & 5,33 \\
SBJ & 0,9397 & 3,00 & 0,6632 & 0,8261 & 0,9234 & 3,33 \\
SES & 0,9047 & 1,00 & 0,5859 & 0,7448 & 0,8439 & 2,00 \\
ZERO & 1,8025 & 8,00 & 1,1078 & 1,3938 & 1,5285 & 8,00 \\
NAIVE & 1,4726 & 7,00 & 1,0262 & 1,3131 & 1,4438 & 7,00 \\
TSB & 0,9232 & 2,00 & 0,5613 & 0,7216 & 0,8203 & 1,00
\end{tabular}

\begin{tabular}{lcccccc} 
EMAE & \multicolumn{2}{c}{ Treino } & \multicolumn{5}{c}{ Teste } \\
Modelo & $\begin{array}{c}\text { a 1 passo } 32 \\
\text { p. }\end{array}$ & $\begin{array}{c}\text { Ranking } \\
\text { treino }\end{array}$ & $\mathrm{T}+1$ & $\mathrm{~T}+3$ & $\mathrm{~T}+6$ & $\begin{array}{c}\text { Ranking } \\
\text { teste }\end{array}$ \\
MMS3 & 0,8531 & 5,00 & 0,6921 & 0,7541 & 0,7956 & 1,67 \\
SBA & 0,8530 & 4,00 & 0,7906 & 0,8119 & 0,8539 & 5,33 \\
CROSTON & 0,8668 & 6,00 & 0,8239 & 0,8393 & 0,8799 & 6,33 \\
SBJ & 0,8523 & 3,00 & 0,7889 & 0,8105 & 0,8525 & 4,00 \\
SES & 0,8256 & 1,00 & 0,7558 & 0,7678 & 0,8096 & 3,00 \\
ZERO & 1,3204 & 8,00 & 0,9303 & 1,0041 & 1,0379 & 8,00 \\
NAIVE & 1,0000 & 7,00 & 0,7899 & 0,8495 & 0,8871 & 6,33 \\
TSB & 0,8429 & 2,00 & 0,7201 & 0,7331 & 0,7748 & 1,33
\end{tabular}

\begin{tabular}{lcccccc} 
PEEE & \multicolumn{2}{c}{ Treino } & \multicolumn{5}{c}{ Teste } \\
Modelo & $\begin{array}{c}\text { a 1 passo 32 } \\
\text { p. }\end{array}$ & $\begin{array}{l}\text { Ranking } \\
\text { treino }\end{array}$ & $\mathrm{T}+1$ & $\mathrm{~T}+3$ & $\mathrm{~T}+6$ & $\begin{array}{c}\text { Ranking } \\
\text { teste }\end{array}$ \\
MMS3 & $-3,1038$ & 3,00 & 0,1817 & 0,6402 & 2,1589 & 2,00 \\
SBA & $-25,1645$ & 6,00 & 0,2605 & 1,1133 & 3,8145 & 6,00 \\
CROSTON & 0,5514 & 2,00 & 0,3108 & 1,4146 & 4,8693 & 7,00 \\
SBJ & $-26,5180$ & 7,00 & 0,2579 & 1,0974 & 3,7589 & 5,00 \\
SES & $-8,4941$ & 4,00 & 0,2423 & 1,0036 & 3,4306 & 4,00 \\
ZERO & $-547,6016$ & 8,00 & $-0,6938$ & $-4,6129$ & $-16,2270$ & 8,00 \\
NAIVE & $-0,3813$ & 1,00 & 0,1634 & 0,5301 & 1,7736 & 1,00 \\
TSB & $-12,5005$ & 5,00 & 0,2038 & 0,7727 & 2,6224 & 3,00
\end{tabular}

\begin{tabular}{lcccccc} 
TAM & \multicolumn{2}{c}{ Treino } & \multicolumn{5}{c}{ Teste } \\
Modelo & $\begin{array}{c}\text { a 1 passo 32 } \\
\text { p. }\end{array}$ & $\begin{array}{c}\text { Ranking } \\
\text { treino }\end{array}$ & $\mathrm{T}+1$ & $\mathrm{~T}+3$ & $\mathrm{~T}+6$ & $\begin{array}{c}\text { Ranking } \\
\text { teste }\end{array}$ \\
MMS3 & 11,0338 & 6,00 & 0,4983 & 1,4852 & 2,9347 & 6,00 \\
SBA & 5,3897 & 2,00 & 0,1383 & 0,4112 & 0,8098 & 1,00 \\
CROSTON & 5,3356 & 1,00 & 0,1385 & 0,4152 & 0,8271 & 2,67 \\
SBJ & 5,4035 & 3,00 & 0,1387 & 0,4121 & 0,8110 & 2,33 \\
SES & 5,4887 & 4,00 & 0,1818 & 0,5300 & 1,0180 & 4,00 \\
ZERO & 33,0354 & 8,00 & 0,9907 & 2,9595 & 5,8682 & 8,00 \\
NAIVE & 19,3904 & 7,00 & 0,6851 & 2,0401 & 4,0350 & 7,00 \\
TSB & 5,8324 & 5,00 & 0,2243 & 0,6544 & 1,2619 & 5,00
\end{tabular}

\begin{tabular}{lcccccc} 
TQM & \multicolumn{2}{c}{ Treino } & \multicolumn{5}{c}{ Teste } \\
Modelo & $\begin{array}{c}\text { a 1 passo 32 } \\
\text { p. }\end{array}$ & $\begin{array}{c}\text { Ranking } \\
\text { treino }\end{array}$ & $\mathrm{T}+1$ & $\mathrm{~T}+3$ & $\mathrm{~T}+6$ & $\begin{array}{c}\text { Ranking } \\
\text { teste }\end{array}$ \\
MMS3 & 7,3822 & 6,00 & 0,4277 & 1,2595 & 2,4536 & 6,00 \\
SBA & 2,0456 & 2,00 & 0,0307 & 0,0915 & 0,1826 & 1,33 \\
CROSTON & 2,1687 & 3,00 & 0,0328 & 0,0989 & 0,2019 & 3,00 \\
SBJ & 2,0444 & 1,00 & 0,0308 & 0,0916 & 0,1825 & 1,67 \\
SES & 2,2023 & 4,00 & 0,0560 & 0,1574 & 0,2902 & 4,00 \\
ZERO & 37,4282 & 8,00 & 0,9821 & 2,9253 & 5,7627 & 8,00 \\
NAIVE & 22,8296 & 7,00 & 0,8950 & 2,6565 & 5,2383 & 7,00 \\
TSB & 2,4114 & 5,00 & 0,0971 & 0,2754 & 0,5106 & 5,00
\end{tabular}

\begin{tabular}{|c|c|c|c|c|c|c|}
\hline PEEEAm & Treino & & & Teste & & \\
\hline Modelo & $\begin{array}{c}\text { a } 1 \text { passo } 32 \\
\text { p. }\end{array}$ & $\begin{array}{c}\text { Ranking } \\
\text { treino }\end{array}$ & $T+1$ & $T+3$ & $T+6$ & $\begin{array}{l}\text { Ranking } \\
\text { teste }\end{array}$ \\
\hline MMS3 & 25,5926 & 2,00 & 0,5604 & 2,8298 & 8,9994 & 2,33 \\
\hline SBA & 135,0345 & 4,00 & 0,6458 & 3,0974 & 9,2756 & 5,33 \\
\hline CROSTON & 135,1593 & 6,00 & 0,6715 & 3,2078 & 9,6568 & 6,33 \\
\hline SBJ & 135,1054 & 5,00 & 0,6445 & 3,0920 & 9,2568 & 4,33 \\
\hline SES & 135,4664 & 7,00 & 0,6106 & 2,8249 & 8,3523 & 2,33 \\
\hline ZERO & 547,6016 & 8,00 & 0,6938 & 4,6129 & 16,2270 & 8,00 \\
\hline NAIVE & 19,0340 & 1,00 & 0,6337 & 3,5306 & 11,7720 & 6,00 \\
\hline TSB & 132,4047 & 3,00 & 0,5786 & 2,6473 & 7,7212 & 1,33 \\
\hline
\end{tabular}

\section{NÃO OTIMIZADO (INÍCIO Pt = MÉDIA )}

\begin{tabular}{lcccccc} 
DMAE & \multicolumn{1}{c}{ Treino } & \multicolumn{5}{c}{ Teste } \\
Modelo & $\begin{array}{c}\text { a 1 passo } 32 \\
\text { p. }\end{array}$ & $\begin{array}{c}\text { Ranking } \\
\text { treino }\end{array}$ & $\mathrm{T}+1$ & $\mathrm{~T}+3$ & $\mathrm{~T}+6$ & $\begin{array}{c}\text { Ranking } \\
\text { teste }\end{array}$ \\
MMS3 & 0,7068 & 6,00 & 0,5604 & 0,6186 & 0,6495 & 1,67 \\
SBA & 0,6933 & 4,00 & 0,6438 & 0,6706 & 0,6988 & 5,33 \\
CROSTON & 0,7046 & 5,00 & 0,6691 & 0,6918 & 0,7189 & 7,00 \\
SBJ & 0,6928 & 3,00 & 0,6425 & 0,6696 & 0,6978 & 4,33 \\
SES & 0,6605 & 1,00 & 0,6104 & 0,6286 & 0,6568 & 3,00 \\
ZERO & 1,0000 & 8,00 & 0,6938 & 0,7638 & 0,7857 & 8,00 \\
NAIVE & 0,8317 & 7,00 & 0,6337 & 0,6914 & 0,7185 & 5,33 \\
TSB & 0,6868 & 2,00 & 0,5786 & 0,5980 & 0,6265 & 1,33
\end{tabular}

$\begin{array}{lcccccc}\text { DQME } & \begin{array}{c}\text { Treino } \\ \text { M 1 passo 32 } 32 \\ \text { p. }\end{array} & \begin{array}{c}\text { Ranking } \\ \text { treino }\end{array} & \begin{array}{c}\mathrm{T}+1 \\ \text { Modelo }\end{array} & \begin{array}{c}\text { Teste } \\ \mathrm{T}+3\end{array} & \mathrm{~T}+6 & \begin{array}{c}\text { Ranking } \\ \text { teste }\end{array} \\ \text { MMS3 } & \mathbf{1 , 0 0 3 3} & 6,00 & 0,6456 & 0,9079 & 1,0281 & 5,00 \\ \text { SBA } & 0,9233 & 4,00 & 0,6610 & 0,8266 & 0,9244 & 4,33 \\ \text { CROSTON } & 0,9349 & 5,00 & 0,6896 & 0,8489 & 0,9447 & 5,33 \\ \text { SBJ } & 0,9228 & 3,00 & 0,6596 & 0,8255 & 0,9235 & 3,33 \\ \text { SES } & 0,8268 & 1,00 & 0,5826 & 0,7451 & 0,8448 & 2,00 \\ \text { ZERO } & 1,8025 & 8,00 & 1,1078 & 1,3938 & 1,5285 & 8,00 \\ \text { NAIVE } & 1,4726 & 7,00 & 1,0262 & 1,3131 & 1,4438 & 7,00 \\ \text { TSB } & 0,9095 & 2,00 & 0,5612 & 0,7216 & 0,8203 & 1,00\end{array}$

$\begin{array}{lcccccc}\text { EMAE } & \begin{array}{c}\text { Treino } \\ \text { M 1 passo } 32\end{array} & \begin{array}{c}\text { Ranking } \\ \text { treino }\end{array} & \begin{array}{c}\mathrm{T}+1 \\ \mathrm{p}\end{array} & \mathrm{T}+3 & \mathrm{~T}+6 & \begin{array}{c}\text { Ranking } \\ \text { teste }\end{array} \\ \text { Modelo } & 0,8531 & 5,00 & 0,6921 & 0,7541 & 0,7956 & 1,67 \\ \text { MMS3 } & 0,8451 & 4,00 & 0,7884 & 0,8112 & 0,8529 & 5,00 \\ \text { SBA } & 0,8578 & 6,00 & 0,8214 & 0,8385 & 0,8790 & 6,33 \\ \text { CROSTON } & 0,8578 & & \\ \text { SBJ } & 0,8446 & 3,00 & 0,7867 & 0,8098 & 0,8516 & 4,00 \\ \text { SES } & 0,8019 & 1,00 & 0,7547 & 0,7674 & 0,8089 & 3,00 \\ \text { ZERO } & 1,3204 & 8,00 & 0,9303 & 1,0041 & 1,0379 & 8,00 \\ \text { NAIVE } & 1,0000 & 7,00 & 0,7899 & 0,8495 & 0,8871 & 6,67 \\ \text { TSB } & 0,8376 & 2,00 & 0,7201 & 0,7330 & 0,7748 & 1,33\end{array}$

\begin{tabular}{lcccccc} 
PEEE & \multicolumn{1}{c}{ Treino } & \multicolumn{5}{c}{ Teste } \\
Modelo & $\begin{array}{c}\text { a 1 passo } 32 \\
\text { p. }\end{array}$ & $\begin{array}{c}\text { Ranking } \\
\text { treino }\end{array}$ & $\mathrm{T}+1$ & $\mathrm{~T}+3$ & $\mathrm{~T}+6$ & $\begin{array}{c}\text { Ranking } \\
\text { teste }\end{array}$ \\
MMS3 & $-3,1038$ & 2,00 & 0,1817 & 0,6402 & 2,1589 & 2,00 \\
SBA & $-38,6950$ & 6,00 & 0,2602 & 1,1114 & 3,8080 & 6,00 \\
CROSTON & $-13,6912$ & 4,00 & 0,3104 & 1,4127 & 4,8625 & 7,00 \\
SBJ & $-40,0110$ & 7,00 & 0,2576 & 1,0956 & 3,7525 & 5,00 \\
SES & $-5,7163$ & 3,00 & 0,2427 & 1,0060 & 3,4391 & 4,00 \\
ZERO & $-547,6016$ & 8,00 & $-0,6938$ & $-4,6129$ & $-16,2270$ & 8,00 \\
NAIVE & $-0,3813$ & 1,00 & 0,1634 & 0,5301 & 1,7736 & 1,00 \\
TSB & $-20,8907$ & 5,00 & 0,2037 & 0,7724 & 2,6214 & 3,00
\end{tabular}

$\begin{array}{lcccccc}\text { TAM } & \begin{array}{c}\text { Treino } \\ \text { a 1 passo } 32 \\ \text { p. }\end{array} & \begin{array}{c}\text { Ranking } \\ \text { treino }\end{array} & \begin{array}{c}\mathrm{T}+1 \\ \text { Modelo }\end{array} & \begin{array}{c}\text { Teste } \\ \mathrm{T}+3\end{array} & \mathrm{~T}+6 & \begin{array}{c}\text { Ranking } \\ \text { teste }\end{array} \\ \text { MMS3 } & 11,0338 & 6,00 & 0,4983 & 1,4852 & 2,9347 & 6,00 \\ \text { SBA } & 5,3303 & 3,00 & 0,1394 & 0,4153 & 0,8177 & 1,00 \\ \text { CROSTON } & 5,1723 & 2,00 & 0,1395 & 0,4185 & 0,8342 & 2,67 \\ \text { SBJ } & 5,3495 & 4,00 & 0,1397 & 0,4162 & 0,8190 & 2,33 \\ \text { SES } & 5,1478 & 1,00 & 0,1856 & 0,5414 & 1,0434 & 4,00 \\ \text { ZERO } & 33,0354 & 8,00 & 0,9907 & 2,9595 & 5,8682 & 8,00 \\ \text { NAIVE } & 19,3904 & 7,00 & 0,6851 & 2,0401 & 4,0350 & 7,00 \\ \text { TSB } & 5,8003 & 5,00 & 0,2244 & 0,6545 & 1,2621 & 5,00\end{array}$

\begin{tabular}{|c|c|c|c|c|c|c|}
\hline TQM & Treino & & & Teste & & \\
\hline Modelo & $\begin{array}{c}\text { a } 1 \text { passo } 32 \\
\text { p. }\end{array}$ & $\begin{array}{l}\text { Ranking } \\
\text { treino }\end{array}$ & $T+1$ & $T+3$ & $T+6$ & $\begin{array}{l}\text { Ranking } \\
\text { teste }\end{array}$ \\
\hline MMS3 & 7,3822 & 6,00 & 0,4277 & 1,2595 & 2,4536 & 6,00 \\
\hline SBA & 1,9112 & 1,00 & 0,0312 & 0,0929 & 0,1854 & 1,33 \\
\hline CROSTON & 1,9328 & 3,00 & 0,0333 & 0,1002 & 0,2048 & 3,00 \\
\hline SBJ & 1,9151 & 2,00 & 0,0312 & 0,0929 & 0,1852 & 1,67 \\
\hline SES & 2,0391 & 4,00 & 0,0584 & 0,1645 & 0,3043 & 4,00 \\
\hline ZERO & 37,4282 & 8,00 & 0,9821 & 2,9253 & 5,7627 & 8,00 \\
\hline NAIVE & 22,8296 & 7,00 & 0,8950 & 2,6565 & 5,2383 & 7,00 \\
\hline TSB & 2,3201 & 5,00 & 0,0972 & 0,2756 & 0,5109 & 5,00 \\
\hline
\end{tabular}

$\begin{array}{lcccccc}\text { PEEEAm } & \begin{array}{c}\text { Treino } \\ \text { a 1 passo } 32 \\ \text { Modelo }\end{array} & \begin{array}{c}\text { Ranking } \\ \text { treino }\end{array} & \mathrm{T}+1 & \mathrm{~T}+3 & \mathrm{~T}+6 & \begin{array}{c}\text { Ranking } \\ \text { teste }\end{array} \\ \text { MMS3 } & 25,5926 & 3,00 & 0,5604 & 2,8298 & 8,9994 & 2,33 \\ \text { SBA } & 128,0454 & 6,00 & 0,6438 & 3,0905 & 9,2929 & 5,33 \\ \text { CROSTON } & 126,2225 & 4,00 & 0,6691 & 3,2010 & 9,6682 & 6,33 \\ \text { SBJ } & 128,2595 & 7,00 & 0,6425 & 3,0850 & 9,2745 & 4,33 \\ \text { SES } & 17,4919 & 1,00 & 0,6104 & 2,8236 & 8,3781 & 2,33 \\ \text { ZERO } & 547,6016 & 8,00 & 0,6938 & 4,6129 & 16,2270 & 8,00 \\ \text { NAIVE } & 19,0340 & 2,00 & 0,6337 & 3,5306 & 11,7720 & 6,00 \\ \text { TSB } & 126,9662 & 5,00 & 0,5786 & 2,6472 & 7,7208 & 1,33\end{array}$


Agência Brasileira ISBN

ISBN: 978-65-86230-76-5 